\title{
Development of $\alpha, \alpha$-Disubstituted Crotylboronate Reagents and Stereoselective Crotylation via Brønsted or Lewis Acid Catalysis
}

\author{
Shang Gao, ${ }^{*, \#}$ Meng Duan, ${ }^{*, \#}$ Qianzhen Shao, ${ }^{*}$ K. N. Houk ${ }^{* t}$ and Ming Chen *t \\ tDepartment of Chemistry and Biochemistry, Auburn University, Auburn, AL 36849 \\ ¥Department of Chemistry and Biochemistry, University of California, Los Angeles, CA \\ 90095 \\ E-mail: mzc0102@auburn.edu \\ E-mail:houk@chem.ucla.edu
}

Supporting Information: Experimental Procedures, Tabulated Spectroscopic Data,

${ }^{1} \mathrm{H}$ and ${ }^{13} \mathrm{C}$ Spectra of New Compounds 
General Experimental Details. All reaction solvents were purified before use. Tetrahydrofuran, dichloromethane, diethyl ether and toluene were purified by passing through a solvent column composed of activated A-1 alumina. Unless indicated otherwise, all reactions were conducted under an atmosphere of argon using flame-dried or oven-dried $\left(120^{\circ} \mathrm{C}\right)$ glassware. The term "concentrated under reduced pressure" refers to the removal of solvents and other volatile materials using a rotary evaporator with the water bath temperature below $30{ }^{\circ} \mathrm{C}$, followed by the removal of residual solvents at high vacuum $(<0.2$ mbar $)$.

Proton nuclear magnetic resonance ( ${ }^{1} \mathrm{H}$ NMR) spectra were acquired on commercial instruments $(400,500$ and $600 \mathrm{MHz})$ at Auburn University NMR facility. Carbon-13 nuclear magnetic resonance $\left({ }^{13} \mathrm{C}\right.$ NMR $)$ spectra were acquired at 101, 126 and $151 \mathrm{MHz}$. The proton signal for the residual non-deuterated solvent $\left(\delta 7.26\right.$ for $\left.\mathrm{CHCl}_{3}\right)$ was used as an internal reference for ${ }^{1} \mathrm{H}$ NMR spectra. For ${ }^{13} \mathrm{C}$ NMR spectra, chemical shifts are reported relative to the $\delta 77.36$ resonance of $\mathrm{CHCl}_{3}$. Coupling constants are reported in $\mathrm{Hz}$. Optical rotations were measured on a Perkin Elmer 241 Automatic Polarimeter. High-resolution mass spectra were recorded on a commercial high-resolution mass spectrometer via the Micro Mass/Analytical Facility operated by the College of Chemistry and Biochemistry, Auburn University.

Analytical thin layer chromatography (TLC) was performed on Kieselgel 60 F254 glass plates precoated with a $0.25 \mathrm{~mm}$ thickness of silica gel. The TLC plates were visualized with UV light and/or by staining with Hanessian solution (ceric sulfate and ammonium molybdate in aqueous sulfuric acid) or $\mathrm{KMnO}_{4}$. Column chromatography was generally performed using Kieselgel 60 (230-400 mesh) silica gel, typically using a 50-100:1 weight ratio of silica gel to crude product. 


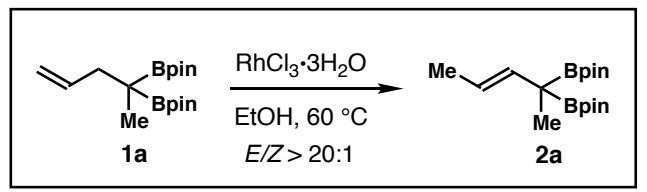

(E)-2,2'-(Pent-3-ene-2,2-diyl)bis(4,4,5,5-tetramethyl-1,3,2-dioxaborolane)

Homoallylic boronic ester $1 \mathrm{a}\left(161 \mathrm{mg}, 0.5 \mathrm{mmol}, 1.0\right.$ equiv) and $\mathrm{RhCl}_{3} \cdot 3 \mathrm{H}_{2} \mathrm{O}(7 \mathrm{mg}, 5$ mol \%) were dissolved in EtOH $(2 \mathrm{~mL})$ under an argon atmosphere. The reaction was stirred at $60{ }^{\circ} \mathrm{C}$ for $2 \mathrm{~h}$. After completion of the reaction, EtOH was removed under reduced pressure and the crude reaction mixture was dissolved in diethyl ether $(10 \mathrm{~mL})$. The solution was washed with brine $(2 \times 5 \mathrm{~mL})$, and dried over anhydrous $\mathrm{Na}_{2} \mathrm{SO}_{4}$. The solvent was removed under reduced pressure and the crude reaction product was purified by flash column chromatography (gradient elution with hexane/ethyl acetate, 20:1 to 5:1) to provide product $\mathbf{2 a}$ in $76 \%$ yield (123 $\mathrm{mg}, E / Z>20: 1)$ as a white solid. When the reaction was conducted in a 3 -mmol scale, the reaction completed in $1 \mathrm{~h}$ and $\mathbf{2 a}$ was isolated in $81 \%$ yield $(782 \mathrm{mg}, E / Z>20: 1)$. ${ }^{1} \mathrm{H} \mathrm{NMR}\left(600 \mathrm{MHz}, \mathrm{CDCl}_{3}\right) \delta 5.81(\mathrm{dq}, J=$ 15.5, $1.5 \mathrm{~Hz}, 1 \mathrm{H}), 5.25$ (dq, $J=15.3,6.3 \mathrm{~Hz}, 1 \mathrm{H}), 1.67$ (dd, $J=6.3,1.4 \mathrm{~Hz}, 3 \mathrm{H}), 1.22$ (s, 12H), $1.21(\mathrm{~s}, 12 \mathrm{H}), 1.17(\mathrm{~s}, 3 \mathrm{H}) .{ }^{13} \mathrm{C} \mathrm{NMR}\left(101 \mathrm{MHz}, \mathrm{CDCl}_{3}\right) \delta$ 134.0, 120.3, 83.4, 25.0, 24.9, 18.8, 15.7. HRMS (ESI $\left.{ }^{+}\right): m / z$ for $\mathrm{C}_{17} \mathrm{H}_{32} \mathrm{~B}_{2} \mathrm{O}_{4} \mathrm{Na}[\mathrm{M}+\mathrm{Na}]^{+}$calcd. 345.2384, found 345.2391 .

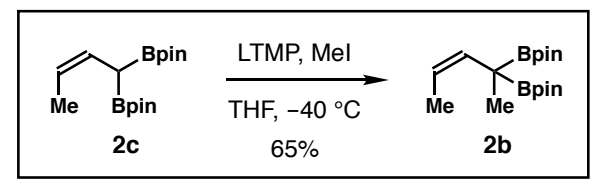

(Z)-2,2'-(Pent-3-ene-2,2-diyl)bis(4,4,5,5-tetramethyl-1,3,2-dioxaborolane) (2b) A round-bottomed flask equipped with a magnetic stir bar were added LiTMP (1.2 mmol, 1.2 equiv) and anhydrous THF ( $5 \mathrm{~mL})$. The mixture was cooled to $-40{ }^{\circ} \mathrm{C}$ and stirred for $5 \mathrm{~min}$. Then a solution of boronate $2 \mathrm{c}(1.0 \mathrm{mmol}, 1.0$ equiv) in THF ( $1 \mathrm{~mL})$ was added and the resulting mixture was stirred at $-40{ }^{\circ} \mathrm{C}$ for $30 \mathrm{~min}$. A solution of methyl iodide (1.5 mmol, 1.5 equiv) in THF ( $1 \mathrm{~mL})$ was added. The reaction mixture was stirred at $-40{ }^{\circ} \mathrm{C}$. After completion of the reaction $(\sim 1 \mathrm{~h})$, a saturated $\mathrm{NH}_{4} \mathrm{Cl}$ solution $(5 \mathrm{~mL})$ was added and the mixture was allowed to warm to ambient temperature. The organic layer was separated and the aqueous layer was extracted with $\mathrm{Et}_{2} \mathrm{O}(3 \times 15 \mathrm{~mL})$. The combined organic extracts were dried over anhydrous sodium sulfate, filtered, and concentrated under reduced pressure. Purification of the crude product by flash chromatography (gradient elution with hexane and EtOAc, 20:1 to 5:1) provided $\mathbf{2 b}$ as a white solid in 65\% 
yield $(Z / E>20: 1) .{ }^{1} \mathrm{H}$ NMR $\left(400 \mathrm{MHz}, \mathrm{CDCl}_{3}\right) \delta 5.72(\mathrm{~d}, J=10.7 \mathrm{~Hz}, 1 \mathrm{H}), 5.43$ (dq, $J$ $=10.8,7.0 \mathrm{~Hz}, 1 \mathrm{H}), 1.60(\mathrm{~d}, J=6.9 \mathrm{~Hz}, 3 \mathrm{H}), 1.21$ (app. s, 27H). ${ }^{13} \mathrm{C} \mathrm{NMR}(151 \mathrm{MHz}$, $\left.\mathrm{CDCl}_{3}\right) \delta 133.9,125.7,83.5,25.02,24.98,18.5,15.5$. HRMS $\left(\mathrm{EI}^{+}\right): m / z$ for $\mathrm{C}_{17} \mathrm{H}_{32} \mathrm{~B}_{2} \mathrm{O}_{4}$ $[\mathrm{M}]^{+}$calcd. 322.2487, found 322.2428.

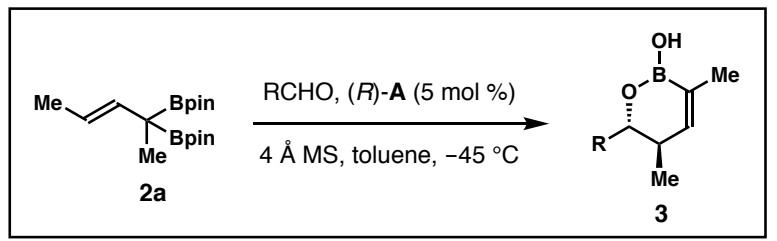

General procedure of CPA-catalyzed allylboration: To a reaction vial containing a stirring bar and freshly activated $4 \AA$ MS (50 mg) was added phosphoric acid $(R)$-A (4 $\mathrm{mg}, 0.005 \mathrm{mmol})$. Toluene $(0.2 \mathrm{~mL})$ was added to the vial followed by dropwise addition of freshly distilled aldehydes $(0.1 \mathrm{mmol}$, if it is a liquid). The mixture was placed in a $-45^{\circ} \mathrm{C}$ cold bath and stirred for $15 \mathrm{~min}$. Allylboronate (42 mg, $0.13 \mathrm{mmol}, 1.3$ equiv) in toluene $(0.1 \mathrm{~mL})$ was added slowly to the reaction mixture via a microliter syringe. The mixture was kept stirring at $-45^{\circ} \mathrm{C}$ and the reaction progress was monitored by ${ }^{1} \mathrm{H}$ NMR analysis. After complete consumption of the aldehyde, the reaction was filtered through silica gel. The filtrate was concentrated under reduced pressure. Purification of the crude product was performed by flash column chromatography (gradient elution with hexane and EtOAc, 30:1 to 10:1) to provide product 3. Enantiopurity of $\mathbf{3}$ was determined by HPLC analysis of the phenylation product derived from Suzuki coupling with PhI, or the vinyl bromide obtained from $\mathrm{Cu}$-mediated bromination.

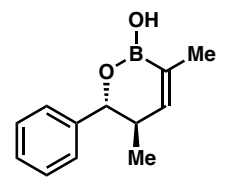

(5R,6R)-3,5-Dimethyl-6-phenyl-5,6-dihydro-2H-1,2-oxaborinin-2-ol (3a) Prepared according to the general procedure. The crude mixture was purified by column chromatography to give the title compound as colorless oil in $89 \%$ yield (18 mg, $E / Z>20: 1)$. Enantiomeric excess was determined by HPLC analysis of the corresponding phenylation product to be $97 \%$ ee $\left(254 \mathrm{~nm}, 25^{\circ} \mathrm{C}\right) ; \mathrm{t}_{1}=$ $6.62 \mathrm{~min}, \mathrm{t}_{2}=7.48 \mathrm{~min}\left[(\right.$ Chiralpak IG) hexane $/ \mathrm{i}-\mathrm{PrOH}, 95: 5,1.0 \mathrm{~mL} / \mathrm{min}] ;[\alpha]_{\mathrm{D}}{ }^{20}=$ $+39.6^{\circ}\left(\mathrm{c} 0.45, \mathrm{CHCl}_{3}\right) ;{ }^{1} \mathrm{H} \mathrm{NMR}\left(600 \mathrm{MHz}, \mathrm{CDCl}_{3}\right) \delta 7.30-7.36(\mathrm{~m}, 5 \mathrm{H}), 6.33(\mathrm{~s}, 1 \mathrm{H})$, $4.58(\mathrm{~d}, J=10.4 \mathrm{~Hz}, 1 \mathrm{H}), 4.11(\mathrm{brs}, 1 \mathrm{H}), 2.57-2.62(\mathrm{~m}, 1 \mathrm{H}), 1.82(\mathrm{~s}, 3 \mathrm{H}), 0.87$ (d, $J=$ $7.0 \mathrm{~Hz}, 3 \mathrm{H}) .{ }^{13} \mathrm{C} \mathrm{NMR}\left(151 \mathrm{MHz}, \mathrm{CDCl}_{3}\right) \delta 150.3,142.1,128.7,128.3,127.5,83.3,39.7$, 18.8, 17.8. HRMS $\left(\mathrm{ESI}^{+}\right): \mathrm{m} / z$ for $\mathrm{C}_{12} \mathrm{H}_{15} \mathrm{BO}_{2} \mathrm{Na}[\mathrm{M}+\mathrm{Na}]^{+}$calcd. 225.1063, found 225.1065 . 


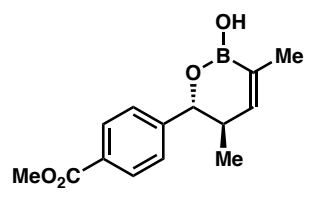

Methyl-4-((5R,6R)-2-hydroxy-3,5-dimethyl-5,6-dihydro-2H-1,2-ox aborinin-6-yl)benzoate (3b) Prepared according to the general procedure. The crude mixture was purified to give the title compound as colorless oil in 92\% yield ( $24 \mathrm{mg}, E / Z>20: 1)$. Enantiomeric excess was determined by HPLC analysis of the corresponding phenylation product to be $99 \%$ ee $\left(254 \mathrm{~nm}, 25{ }^{\circ} \mathrm{C}\right) ; \mathrm{t}_{1}=14.0 \mathrm{~min}, \mathrm{t}_{2}=15.2 \mathrm{~min}$ [(Chiralpak IB) hexane/i-PrOH, 95:5, $1.0 \mathrm{~mL} / \mathrm{min}] ;[\alpha]_{\mathrm{D}}{ }^{20}=+59.3^{\circ}\left(\mathrm{c} 0.60, \mathrm{CHCl}_{3}\right) ;{ }^{1} \mathrm{H} \mathrm{NMR}\left(600 \mathrm{MHz}, \mathrm{CDCl}_{3}\right) \delta 8.03$ $(\mathrm{d}, J=8.1 \mathrm{~Hz}, 2 \mathrm{H}), 7.41$ (d, $J=8.2 \mathrm{~Hz}, 2 \mathrm{H}), 6.31(\mathrm{~s}, 1 \mathrm{H}), 4.64$ (d, $J=10.4 \mathrm{~Hz}, 1 \mathrm{H}), 4.27$ $(\mathrm{s}, 1 \mathrm{H}), 3.92(\mathrm{~s}, 3 \mathrm{H}), 2.53-2.59(\mathrm{~m}, 1 \mathrm{H}), 1.82(\mathrm{~s}, 3 \mathrm{H}), 0.87(\mathrm{~d}, J=7.2 \mathrm{~Hz}, 3 \mathrm{H}) .{ }^{13} \mathrm{C}$ NMR $\left(151 \mathrm{MHz}, \mathrm{CDCl}_{3}\right) \delta 167.3,150.0,147.1,129.95,129.90,127.4,82.7,52.6,39.7$, 18.9, 17.6. HRMS (ESI $\left.{ }^{+}\right): m / z$ for $\mathrm{C}_{14} \mathrm{H}_{18} \mathrm{BO}_{4}[\mathrm{M}+\mathrm{H}]^{+}$calcd. 261.1298, found 261.1296.

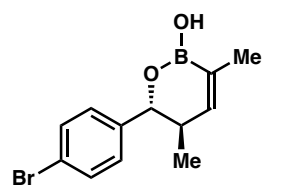

\section{(5R,6R)-6-(4-Bromophenyl)-3,5-dimethyl-5,6-dihydro-2H-1,2-oxabo} rinin-2-ol (3c) Prepared according to the general procedure. The crude mixture was purified by column chromatography to give the title compound as colorless oil in $86 \%$ yield (24 mg, $E / Z>20: 1)$. Enantiomeric excess was determined by HPLC analysis of the corresponding phenylation product to be $99 \%$ ee $\left(254 \mathrm{~nm}, 25^{\circ} \mathrm{C}\right) ; \mathrm{t}_{1}=4.60 \mathrm{~min}, \mathrm{t}_{2}=5.75 \mathrm{~min}$ [(Chiralpak IB) hexane/i-PrOH, 95:5, 1.0 $\mathrm{mL} / \mathrm{min}] ;[\alpha]_{\mathrm{D}}{ }^{20}=+87.0^{\circ}\left(\mathrm{c} 0.60, \mathrm{CHCl}_{3}\right) ;{ }^{1} \mathrm{H} \mathrm{NMR}\left(600 \mathrm{MHz}, \mathrm{CDCl}_{3}\right) \delta 7.48(\mathrm{~d}, J=8.4$ Hz, 2H), 7.22 (d, $J=8.4 \mathrm{~Hz}, 2 \mathrm{H}), 6.31(\mathrm{~s}, 1 \mathrm{H}), 4.54(\mathrm{~d}, J=10.5 \mathrm{~Hz}, 1 \mathrm{H}), 4.11(\mathrm{~s}, 1 \mathrm{H})$, $2.50-2.56(\mathrm{~m}, 1 \mathrm{H}), 1.81(\mathrm{~s}, 3 \mathrm{H}), 0.86(\mathrm{~d}, J=7.2 \mathrm{~Hz}, 3 \mathrm{H}) .{ }^{13} \mathrm{C} \mathrm{NMR}\left(151 \mathrm{MHz}, \mathrm{CDCl}_{3}\right)$ $\delta 150.1,141.1,131.7,129.2,122.1,82.6,39.7,18.9,17.6$. HRMS $\left(\mathrm{ESI}^{+}\right): \mathrm{m} / z$ for $\mathrm{C}_{12} \mathrm{H}_{14} \mathrm{BO}_{2} \mathrm{BrNa}[\mathrm{M}+\mathrm{Na}]^{+}$calcd. 303.0168, found 303.0166.

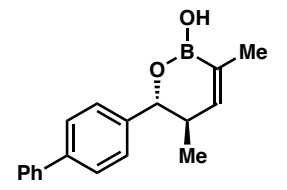

(5R,6R)-6-([1,1'-Biphenyl]-4-yl)-3,5-dimethyl-5,6-dihydro-2H-1,2-ox aborinin-2-ol (3d) Prepared according to the general procedure. The crude mixture was purified by column chromatography to give the title compound as colorless oil in $97 \%$ yield (27 mg, $E / Z>20: 1)$. Enantiomeric excess was determined by HPLC analysis of the corresponding phenylation product to be $>99 \%$ ee $\left(254 \mathrm{~nm}, 25^{\circ} \mathrm{C}\right) ; \mathrm{t}_{1}=13.3 \mathrm{~min}, \mathrm{t}_{2}=14.4 \mathrm{~min}$ [(Chiralpak IE) hexane/i-PrOH, 98:2, 1.0 $\mathrm{mL} / \mathrm{min}] ;[\alpha]_{\mathrm{D}}{ }^{20}=+91.7^{\circ}\left(\mathrm{c} 0.65, \mathrm{CHCl}_{3}\right) ;{ }^{1} \mathrm{H} \mathrm{NMR}\left(600 \mathrm{MHz}, \mathrm{CDCl}_{3}\right) \delta 7.59$ (app. d, J $=7.5 \mathrm{~Hz}, 4 \mathrm{H}), 7.44(\mathrm{dd}, J=7.5,7.5 \mathrm{~Hz}, 2 \mathrm{H}), 7.41(\mathrm{~d}, J=7.5 \mathrm{~Hz}, 2 \mathrm{H}), 7.35$ (dd, $J=7.3$, $7.3 \mathrm{~Hz}, 1 \mathrm{H}), 6.35(\mathrm{~s}, 1 \mathrm{H}), 4.64(\mathrm{~d}, J=10.5 \mathrm{~Hz}, 1 \mathrm{H}), 4.09(\mathrm{~s}, 1 \mathrm{H}), 2.63-2.68(\mathrm{~m}, 1 \mathrm{H})$, $1.84(\mathrm{~s}, 3 \mathrm{H}), 0.93(\mathrm{~d}, J=7.2 \mathrm{~Hz}, 3 \mathrm{H}) .{ }^{13} \mathrm{C} \mathrm{NMR}\left(151 \mathrm{MHz}, \mathrm{CDCl}_{3}\right) \delta 150.3,141.23$, 141.20, 141.1, 129.1, 127.9, 127.6, 127.5 (two overlapping carbon signals), 83.0, 39.6, 18.8, 17.9. HRMS $\left(\mathrm{ESI}^{+}\right): m / z$ for $\mathrm{C}_{18} \mathrm{H}_{20} \mathrm{BO}_{2}[\mathrm{M}+\mathrm{H}]^{+}$calcd. 279.1556, found 279.1548. 


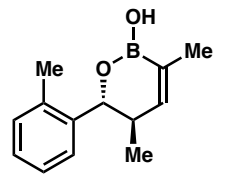

(5R,6R)-3,5-Dimethyl-6-(o-tolyl)-5,6-dihydro-2H-1,2-oxaborinin-2-ol

(3e) Prepared according to the general procedure with $10 \mathrm{~mol} \%(R)$-A.

The crude mixture was purified to give the product as colorless oil in $88 \%$ yield (19 mg, $E / Z>20: 1)$. Enantiomeric excess was determined by HPLC analysis of the corresponding phenylation product to be $90 \%$ ee $\left(254 \mathrm{~nm}, 25^{\circ} \mathrm{C}\right) ; \mathrm{t}_{1}=5.06 \mathrm{~min}, \mathrm{t}_{2}=5.55$ $\min \left[(\right.$ Chiralpak IC) hexane/i-PrOH, 95:5, $1.0 \mathrm{~mL} / \mathrm{min}] ;[\alpha]_{\mathrm{D}}^{20}=+41.6^{\circ}\left(\mathrm{c} 0.50, \mathrm{CHCl}_{3}\right)$; ${ }^{1} \mathrm{H}$ NMR $\left(600 \mathrm{MHz}, \mathrm{CDCl}_{3}\right) \delta 7.37(\mathrm{~d}, J=7.2 \mathrm{~Hz}, 1 \mathrm{H}), 7.23(\mathrm{dd}, J=7.1,6.8 \mathrm{~Hz}, 1 \mathrm{H})$, $7.15-7.21(\mathrm{~m}, 2 \mathrm{H}), 6.35(\mathrm{~s}, 1 \mathrm{H}), 4.94(\mathrm{~d}, J=10.5 \mathrm{~Hz}, 1 \mathrm{H}), 4.04(\mathrm{~s}, 1 \mathrm{H}), 2.69-2.76(\mathrm{~m}$, $1 \mathrm{H}), 2.37(\mathrm{~s}, 3 \mathrm{H}), 1.83(\mathrm{~s}, 3 \mathrm{H}), 0.89(\mathrm{~d}, J=7.2 \mathrm{~Hz}, 3 \mathrm{H}) .{ }^{13} \mathrm{C} \mathrm{NMR}\left(151 \mathrm{MHz}, \mathrm{CDCl}_{3}\right) \delta$ $150.4,140.0,136.1,130.8,128.0,127.2,126.5,78.9,39.4,20.0,18.9,17.5$. HRMS $\left(\mathrm{ESI}^{+}\right): \mathrm{m} / z$ for $\mathrm{C}_{13} \mathrm{H}_{17} \mathrm{BO}_{2} \mathrm{Na}[\mathrm{M}+\mathrm{Na}]^{+}$calcd. 239.1219, found 239.1214.

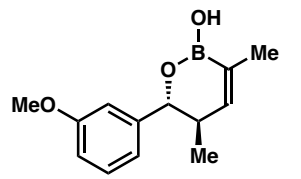

(5R,6R)-6-(3-Methoxyphenyl)-3,5-dimethyl-5,6-dihydro-2H-1,2-ox aborinin-2-ol (3f) Prepared according to the general procedure. The crude mixture was purified by column chromatography to give the title compound as colorless oil in 95\% yield (22 $\mathrm{mg}, E / Z>20: 1)$. Enantiomeric excess was determined by HPLC analysis of the corresponding phenylation product to be $>99 \%$ ee $\left(254 \mathrm{~nm}, 25^{\circ} \mathrm{C}\right) ; \mathrm{t}_{1}=5.18 \mathrm{~min}, \mathrm{t}_{2}=5.64 \mathrm{~min}$ [(Chiralpak IC) hexane/i-PrOH, 95:5, 1.0 $\mathrm{mL} / \mathrm{min}] ;[\alpha]_{\mathrm{D}}{ }^{20}=+109.8^{\circ}\left(\mathrm{c} 0.55, \mathrm{CHCl}_{3}\right) ;{ }^{1} \mathrm{H} \mathrm{NMR}\left(600 \mathrm{MHz}, \mathrm{CDCl}_{3}\right) \delta 7.27$ (dd, $J=$ 7.9, $7.5 \mathrm{~Hz}, 1 \mathrm{H}), 6.92(\mathrm{~d}, J=7.6 \mathrm{~Hz}, 1 \mathrm{H}), 6.89(\mathrm{~s}, 1 \mathrm{H}), 6.85(\mathrm{dd}, J=7.9,2.2 \mathrm{~Hz}, 1 \mathrm{H})$, $6.32(\mathrm{~s}, 1 \mathrm{H}), 4.55(\mathrm{~d}, J=10.5 \mathrm{~Hz}, 1 \mathrm{H}), 4.17(\mathrm{~s}, 1 \mathrm{H}), 3.82(\mathrm{~s}, 3 \mathrm{H}), 2.56-2.63(\mathrm{~m}, 1 \mathrm{H})$, $1.81(\mathrm{~s}, 3 \mathrm{H}), 0.87(\mathrm{~d}, J=7.2 \mathrm{~Hz}, 3 \mathrm{H}) .{ }^{13} \mathrm{C} \mathrm{NMR}\left(151 \mathrm{MHz}, \mathrm{CDCl}_{3}\right) \delta 159.8,150.3,143.5$, 129.7, 119.9, 113.6, 112.9, 83.2, 55.6, 39.5, 18.9, 17.7. HRMS $\left(\mathrm{ESI}^{+}\right): \mathrm{m} / \mathrm{z}$ for $\mathrm{C}_{13} \mathrm{H}_{17} \mathrm{BO}_{3} \mathrm{Na}[\mathrm{M}+\mathrm{Na}]^{+}$calcd. 255.1168, found 255.1160.

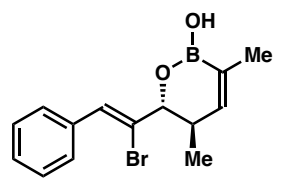

(5R,6R)-6-((Z)-1-Bromo-2-phenylvinyl)-3,5-dimethyl-5,6-dihydro-2 $\boldsymbol{H}-1,2-0 x a b o r i n i n-2-01$ (3g) Prepared according to the general procedure to give the title compound as colorless oil in $81 \%$ yield ( 25 $\mathrm{mg}, E / Z>20: 1)$. Enantiomeric excess was determined by HPLC analysis of the corresponding bromination product to be $>99 \%$ ee $\left(254 \mathrm{~nm}, 25^{\circ} \mathrm{C}\right) ; \mathrm{t}_{1}=5.62 \mathrm{~min}, \mathrm{t}_{2}=$ $8.04 \min \left[(\right.$ Chiralpak ID) hexane/i-PrOH, 95:5, $1.0 \mathrm{~mL} / \mathrm{min}] ;[\alpha]_{\mathrm{D}}{ }^{20}=-102.3^{\circ}$ (c 0.60, $\left.\mathrm{CHCl}_{3}\right) ;{ }^{1} \mathrm{H} \mathrm{NMR}\left(600 \mathrm{MHz}, \mathrm{CDCl}_{3}\right) \delta 7.64(\mathrm{~d}, J=7.8 \mathrm{~Hz}, 2 \mathrm{H}), 7.37(\mathrm{dd}, J=8.3,6.7 \mathrm{~Hz}$, 2H), $7.31-7.34$ (m, 1H), 7.02 (s, 1H), $6.31(\mathrm{~s}, 1 \mathrm{H}), 4.27$ (s, 1H), 4.24 (d, $J=10.4 \mathrm{~Hz}$, $1 \mathrm{H}), 2.84-2.91(\mathrm{~m}, 1 \mathrm{H}), 1.80(\mathrm{~s}, 3 \mathrm{H}), 1.01(\mathrm{~d}, J=7.2 \mathrm{~Hz}, 3 \mathrm{H}) .{ }^{13} \mathrm{C}$ NMR $(151 \mathrm{MHz}$, $\left.\mathrm{CDCl}_{3}\right) \delta 149.7,135.1,131.7,129.6,128.7,128.5,127.1,86.2,36.0,18.8,17.5$. HRMS $\left(\mathrm{ESI}^{+}\right): \mathrm{m} / z$ for $\mathrm{C}_{14} \mathrm{H}_{16} \mathrm{BBrO}_{2} \mathrm{Na}[\mathrm{M}+\mathrm{Na}]^{+}$calcd. 329.0324, found 329.0318 . 


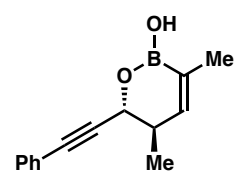

(5R,6R)-3,5-Dimethyl-6-(phenylethynyl)-5,6-dihydro-2H-1,2-oxaborin in-2-ol (3h) Prepared according to the general procedure to give the title compound as colorless oil in $93 \%$ yield ( $21 \mathrm{mg}, E / Z>20: 1$ ). Enantiomeric excess was determined by HPLC analysis of the corresponding phenylation product to be $98 \%$ ee $\left(254 \mathrm{~nm}, 25{ }^{\circ} \mathrm{C}\right) ; \mathrm{t}_{1}=6.65 \mathrm{~min}, \mathrm{t}_{2}=9.29 \mathrm{~min}$ [(Chiralpak IC) hexane/i-PrOH, 95:5, $1.0 \mathrm{~mL} / \mathrm{min}] ;[\alpha]_{\mathrm{D}}{ }^{20}=+31.6^{\circ}\left(\mathrm{c} 0.50, \mathrm{CHCl}_{3}\right) ;{ }^{1} \mathrm{H} \mathrm{NMR}(600 \mathrm{MHz}$, $\left.\mathrm{CDCl}_{3}\right) \delta 7.45-7.47(\mathrm{~m}, 2 \mathrm{H}), 7.26-7.32(\mathrm{~m}, 3 \mathrm{H}), 6.32(\mathrm{~s}, 1 \mathrm{H}), 4.59(\mathrm{~d}, J=10.1 \mathrm{~Hz}$, $1 \mathrm{H}), 4.24(\mathrm{~s}, 1 \mathrm{H}), 2.68-2.71(\mathrm{~m}, 1 \mathrm{H}), 1.79(\mathrm{~s}, 3 \mathrm{H}), 1.18(\mathrm{~d}, J=7.2 \mathrm{~Hz}, 3 \mathrm{H}) .{ }^{13} \mathrm{C}$ NMR $\left(151 \mathrm{MHz}, \mathrm{CDCl}_{3}\right) \delta 149.5,132.1,128.9,128.6,122.6,88.2,85.3,71.1,39.1,18.9,18.0$. HRMS (ESI ${ }^{+}$): $m / z$ for $\mathrm{C}_{14} \mathrm{H}_{16} \mathrm{BO}_{2}[\mathrm{M}+\mathrm{H}]^{+}$calcd. 227.1243, found 227.1260.

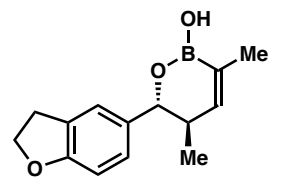

(5R,6R)-6-(2,3-Dihydrobenzofuran-5-yl)-3,5-dimethyl-5,6-dihydro-2 $H$-1,2-oxaborinin-2-ol (3i) Prepared according to the general procedure. The crude mixture was purified by column chromatography to give the title compound as colorless oil in $94 \%$ yield (23 mg, $E / Z>20: 1$ ). Enantiomeric excess was determined by HPLC analysis of the corresponding phenylation product to be $96 \%$ ee $\left(254 \mathrm{~nm}, 25{ }^{\circ} \mathrm{C}\right) ; \mathrm{t}_{1}=9.23 \mathrm{~min}, \mathrm{t}_{2}=11.6 \mathrm{~min}$ [(Chiralpak IC) hexane/i-PrOH, 95:5, $1.0 \mathrm{~mL} / \mathrm{min}] ;[\alpha]_{\mathrm{D}}{ }^{20}=+108.4^{\circ}\left(\mathrm{c} 0.55, \mathrm{CHCl}_{3}\right) ;{ }^{1} \mathrm{H} \mathrm{NMR}(600 \mathrm{MHz}$, $\left.\mathrm{CDCl}_{3}\right) \delta 7.20(\mathrm{~s}, 1 \mathrm{H}), 7.05(\mathrm{~d}, J=8.0 \mathrm{~Hz}, 1 \mathrm{H}), 6.75(\mathrm{~d}, J=8.2 \mathrm{~Hz}, 1 \mathrm{H}), 6.33(\mathrm{~s}, 1 \mathrm{H})$, $4.57(\mathrm{t}, J=8.7 \mathrm{~Hz}, 2 \mathrm{H}), 4.50(\mathrm{~d}, J=10.7 \mathrm{~Hz}, 1 \mathrm{H}), 4.04(\mathrm{~s}, 1 \mathrm{H}), 3.21(\mathrm{t}, J=8.7 \mathrm{~Hz}, 2 \mathrm{H})$, $2.57-2.61(\mathrm{~m}, 1 \mathrm{H}), 1.82(\mathrm{~s}, 3 \mathrm{H}), 0.86(\mathrm{~d}, J=7.2 \mathrm{~Hz}, 3 \mathrm{H}) .{ }^{13} \mathrm{C} \mathrm{NMR}\left(126 \mathrm{MHz}, \mathrm{CDCl}_{3}\right)$ $\delta 160.2,150.5,134.3,127.7,127.6,123.9,109.2,83.2,71.7,39.7,30.1,18.8,17.8$. HRMS (ESI ${ }^{+}$): $m / z$ for $\mathrm{C}_{14} \mathrm{H}_{17} \mathrm{BO}_{3} \mathrm{Na}[\mathrm{M}+\mathrm{Na}]^{+}$calcd. 267.1168, found 267.1168.

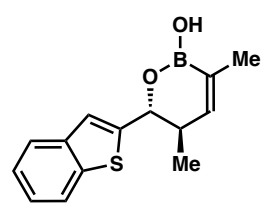

(5R,6R)-6-(Benzo[b]thiophen-2-yl)-3,5-dimethyl-5,6-dihydro-2H-1,2 -oxaborinin-2-ol (3j) Prepared according to the general procedure. The crude mixture was purified by column chromatography to give the title compound as colorless oil in $93 \%$ yield (24 mg, $E / Z>20: 1$ ).

Enantiomeric excess was determined by HPLC analysis of the corresponding phenylation product to be $>99 \%$ ee $\left(254 \mathrm{~nm}, 25{ }^{\circ} \mathrm{C}\right) ; \mathrm{t}_{1}=8.06 \mathrm{~min}, \mathrm{t}_{2}=8.72 \mathrm{~min}$ [(Chiralpak IB) hexane/i-PrOH, 95:5, $1.0 \mathrm{~mL} / \mathrm{min}] ;[\alpha]_{\mathrm{D}}{ }^{20}=+10.0^{\circ}\left(\mathrm{c} 0.60, \mathrm{CHCl}_{3}\right) ;{ }^{1} \mathrm{H} \mathrm{NMR}(600 \mathrm{MHz}$, $\left.\mathrm{CDCl}_{3}\right) \delta 7.82(\mathrm{~d}, J=7.8 \mathrm{~Hz}, 1 \mathrm{H}), 7.73(\mathrm{~d}, J=7.9 \mathrm{~Hz}, 1 \mathrm{H}), 7.30-7.36(\mathrm{~m}, 2 \mathrm{H}), 7.22(\mathrm{~s}$, 1H), 6.37 (s, 1H), 4.96 (d, J=9.4 Hz, 1H), 4.25 (s, 1H), $2.70-2.77$ (m, 1H), $1.82(\mathrm{~s}, 3 \mathrm{H})$, $1.01(\mathrm{~d}, J=7.1 \mathrm{~Hz}, 3 \mathrm{H}) .{ }^{13} \mathrm{C}$ NMR $\left(151 \mathrm{MHz}, \mathrm{CDCl}_{3}\right) \delta 149.6,146.3,139.9,139.4$, $124.7,124.6,123.9,122.8,122.5,78.9,39.8,18.9,18.2$. HRMS $\left(\mathrm{ESI}^{+}\right): \mathrm{m} / \mathrm{z}$ for $\mathrm{C}_{14} \mathrm{H}_{15} \mathrm{BO}_{2} \mathrm{SNa}[\mathrm{M}+\mathrm{Na}]^{+}$calcd. 281.0784, found 281.0770. 


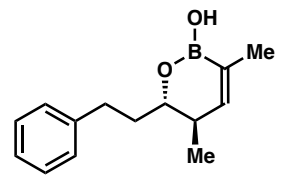

(5R,6S)-3,5-Dimethyl-6-phenethyl-5,6-dihydro-2H-1,2-oxaborinin-2

-ol (3k) Prepared according to the general procedure with $10 \mathrm{~mol} \%$

$(R)$-B as the catalyst. The crude mixture was purified to give the title compound as colorless oil in $87 \%$ yield $(20 \mathrm{mg}, E / Z>20: 1)$. Enantiomeric excess was determined by HPLC analysis of the corresponding bromination product to be $95 \%$ ee $\left(254 \mathrm{~nm}, 25^{\circ} \mathrm{C}\right) ; \mathrm{t}_{1}=12.2 \mathrm{~min}, \mathrm{t}_{2}=12.8 \mathrm{~min}$ [(Chiralpak IA) hexane/i-PrOH, 98:2, 1.0 $\mathrm{mL} / \mathrm{min}] ;[\alpha]_{\mathrm{D}}{ }^{20}=-28.8^{\circ}\left(\mathrm{c} 0.50, \mathrm{CHCl}_{3}\right) ;{ }^{1} \mathrm{H} \mathrm{NMR}\left(600 \mathrm{MHz}, \mathrm{CDCl}_{3}\right) \delta 7.26-7.30(\mathrm{~m}$, $2 \mathrm{H}), 7.17-7.22(\mathrm{~m}, 3 \mathrm{H}), 6.25(\mathrm{~s}, 1 \mathrm{H}), 4.02(\mathrm{~s}, 1 \mathrm{H}), 3.65-3.68(\mathrm{~m}, 1 \mathrm{H}), 2.87$ (ddd, $J=$ 13.1, 10.3, $5.0 \mathrm{~Hz}, 1 \mathrm{H}), 2.67$ (ddd, $J=13.6,10.1,6.9 \mathrm{~Hz}, 1 \mathrm{H}), 2.26-2.30(\mathrm{~m}, 1 \mathrm{H}), 1.78$ - $1.90(\mathrm{~m}, 2 \mathrm{H}), 1.76(\mathrm{~s}, 3 \mathrm{H}), 0.99(\mathrm{~d}, J=7.2 \mathrm{~Hz}, 3 \mathrm{H}) .{ }^{13} \mathrm{C} \mathrm{NMR}\left(151 \mathrm{MHz}, \mathrm{CDCl}_{3}\right) \delta$ 150.3, 142.7, 128.8, 128.7, 126.1, 79.2, 37.6, 37.1, 32.0, 18.82, 18.77. HRMS (ESI $\left.{ }^{+}\right): m / z$ for $\mathrm{C}_{14} \mathrm{H}_{20} \mathrm{BO}_{2}[\mathrm{M}+\mathrm{H}]^{+}$calcd. 231.1556, found 231.1565

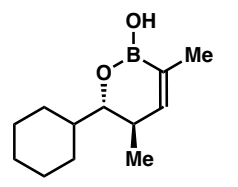

(5R,6S)-6-Cyclohexyl-3,5-dimethyl-5,6-dihydro-2H-1,2-oxaborinin-2-ol

(3l) Prepared according to the general procedure with $10 \mathrm{~mol} \%(R)-\mathbf{B}$ as the catalyst. The crude mixture was purified by column chromatography to give the title compound as colorless oil in $82 \%$ yield (17 mg, $E / Z>20: 1)$. Enantiomeric excess was determined by HPLC analysis of the corresponding phenylation product to be $98 \%$ ee $\left(254 \mathrm{~nm}, 25^{\circ} \mathrm{C}\right) ; \mathrm{t}_{1}=7.20 \mathrm{~min}, \mathrm{t}_{2}=7.72 \mathrm{~min}[($ Chiralpak IG) hexane/i-PrOH, 98:2, $1.0 \mathrm{~mL} / \mathrm{min}] ;[\alpha]_{\mathrm{D}}{ }^{20}=-37.5^{\circ}\left(\mathrm{c} 0.40, \mathrm{CHCl}_{3}\right) ;{ }^{1} \mathrm{H}$ NMR $\left(500 \mathrm{MHz}, \mathrm{CDCl}_{3}\right) \delta 6.26$ (s, $1 \mathrm{H}), 3.88(\mathrm{~s}, 1 \mathrm{H}), 3.47(\mathrm{dd}, J=7.9,3.7 \mathrm{~Hz}, 1 \mathrm{H}), 2.39-2.45(\mathrm{~m}, 1 \mathrm{H}), 1.74-1.77(\mathrm{~m}$, $5 \mathrm{H}), 1.61-1.68(\mathrm{~m}, 2 \mathrm{H}), 1.42-1.52(\mathrm{~m}, 2 \mathrm{H}), 1.31-1.38(\mathrm{~m}, 1 \mathrm{H}), 1.07-1.28(\mathrm{~m}, 4 \mathrm{H})$, $1.00(\mathrm{~d}, J=7.1 \mathrm{~Hz}, 3 \mathrm{H}) .{ }^{13} \mathrm{C}$ NMR $\left(126 \mathrm{MHz}, \mathrm{CDCl}_{3}\right) \delta 150.6,84.1,40.7,34.1,30.7$, 26.91, 26.89, 26.88, 26.7, 19.1, 18.8. HRMS $\left(\mathrm{ESI}^{+}\right): m / z$ for $\mathrm{C}_{12} \mathrm{H}_{21} \mathrm{BO}_{2}[\mathrm{M}+\mathrm{H}]^{+}$calcd. 209.1713 , found 209.1716 .

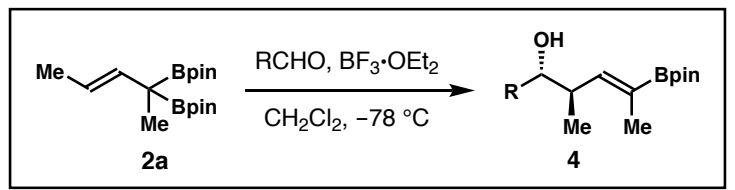

General procedure for the allylboration of aldehydes: To a reaction flask containing a stirring bar were added allylboronate $2 \mathrm{a}(42 \mathrm{mg}, 0.13 \mathrm{mmol})$ and dichloromethane (2 $\mathrm{mL}$ ). The mixture was cooled in a $-78^{\circ} \mathrm{C}$ cold bath and stirred for $15 \mathrm{~min}$, and $\mathrm{BF}_{3} \cdot \mathrm{OEt}_{2}$ $\left(0.5 \mathrm{M}\right.$ in $\left.\mathrm{CH}_{2} \mathrm{Cl}_{2}, 40 \mu \mathrm{L}, 0.02 \mathrm{mmol}\right)$ was added. After stirring for $5 \mathrm{~min}$ at $-78{ }^{\circ} \mathrm{C}$, freshly distilled aldehyde $(0.1 \mathrm{mmol}$, if it is a liquid) was added slowly to the reaction mixture via a microliter syringe. The mixture was kept at $-78{ }^{\circ} \mathrm{C}$ and stirred until 
complete consumption of the aldehyde (typically after $12 \mathrm{~h}$ ). Then saturated aqueous sodium bicarbonate solution $(1 \mathrm{~mL})$ and diethyl ether $(10 \mathrm{~mL})$ were added. The reaction mixture was allowed to warm to ambient temperature. Brine $(5 \mathrm{~mL})$ was added and the resulting mixture was stirred for another $3 \mathrm{~h}$. Then the organic layer was separated and the aqueous layer was extracted with $\mathrm{Et}_{2} \mathrm{O}(3 \times 5 \mathrm{~mL})$. The combined organic extracts were dried over anhydrous sodium sulfate, filtered, and concentrated under reduced pressure. Purification of the crude product by flash column chromatography (gradient elution with hexane and ethyl acetate, 20:1 to 5:1) provided homoallylic alcohol 4.

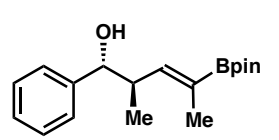

rac-(1R,2R,Z)-2-Methyl-1-phenyl-4-(4,4,5,5-tetramethyl-1,3,2-dioxa borolan-2-yl)pent-3-en-1-ol (4a) Prepared according to the general procedure to give the title compound as colorless oil in $93 \%$ yield (28 $\mathrm{mg}, Z / E>20: 1) .{ }^{1} \mathrm{H} \mathrm{NMR}\left(600 \mathrm{MHz}, \mathrm{CDCl}_{3}\right) \delta 7.33-7.35(\mathrm{~m}, 4 \mathrm{H}), 7.26-7.29(\mathrm{~m}, 1 \mathrm{H})$, $6.25(\mathrm{~d}, J=9.5 \mathrm{~Hz}, 1 \mathrm{H}), 4.34$ (d, $J=8.8 \mathrm{~Hz}, 1 \mathrm{H}), 2.87-2.93$ (m, 1H), 2.08 (brs, 1H), $1.78(\mathrm{~s}, 3 \mathrm{H}), 1.28(\mathrm{~s}, 12 \mathrm{H}), 0.76(\mathrm{~d}, J=6.6 \mathrm{~Hz}, 3 \mathrm{H}) .{ }^{13} \mathrm{C} \mathrm{NMR}\left(151 \mathrm{MHz}, \mathrm{CDCl}_{3}\right) \delta 148.0$, 142.6, 128.6, 128.1, 127.4, 83.7, 79.1, 41.8, 25.2, 25.1, 16.9, 14.9. HRMS (ESI $\left.{ }^{+}\right): m / z$ for $\mathrm{C}_{18} \mathrm{H}_{27} \mathrm{BO}_{3} \mathrm{Na}[\mathrm{M}+\mathrm{Na}]^{+}$calcd. 325.1951, found 325.1945.

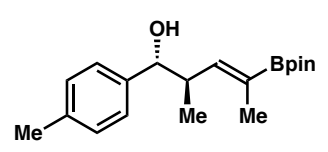

rac-(1R,2R,Z)-2-Methyl-4-(4,4,5,5-tetramethyl-1,3,2-dioxaborola n-2-yl)-1-(p-tolyl)pent-3-en-1-ol (4b) Prepared according to the general procedure. The crude mixture was purified by column chromatography to give the title compound as colorless oil in $85 \%$ yield (27 $\mathrm{mg}, Z / E>$ 20:1). ${ }^{1} \mathrm{H}$ NMR (600 MHz, $\left.\mathrm{CDCl}_{3}\right) \delta 7.25(\mathrm{~d}, J=7.7 \mathrm{~Hz}, 2 \mathrm{H}), 7.15(\mathrm{~d}, J=7.4 \mathrm{~Hz}, 2 \mathrm{H})$, $6.25(\mathrm{~d}, J=9.4 \mathrm{~Hz}, 1 \mathrm{H}), 4.30(\mathrm{~d}, J=8.8 \mathrm{~Hz}, 1 \mathrm{H}), 2.86-2.92(\mathrm{~m}, 1 \mathrm{H}), 2.34(\mathrm{~s}, 3 \mathrm{H}), 1.82$ (br, $1 \mathrm{H}), 1.78(\mathrm{~s}, 3 \mathrm{H}), 1.28(\mathrm{~s}, 12 \mathrm{H}), 0.75(\mathrm{~d}, J=6.6 \mathrm{~Hz}, 3 \mathrm{H}) .{ }^{13} \mathrm{C}$ NMR $(151 \mathrm{MHz}$, $\left.\mathrm{CDCl}_{3}\right) \delta 148.2,139.6,137.7,129.3,127.3,83.7,78.9,41.7,25.2,25.1,21.5,16.9,14.9$. HRMS (ESI ${ }^{+}$): $m / z$ for $\mathrm{C}_{19} \mathrm{H}_{33} \mathrm{BNO}_{3}\left[\mathrm{M}+\mathrm{NH}_{4}\right]^{+}$calcd. 334.2553, found 334.2550.

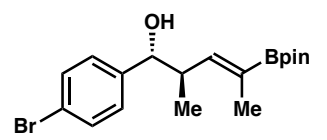

rac-(1R,2R,Z)-1-(4-Bromophenyl)-2-methyl-4-(4,4,5,5-tetramethy l-1,3,2-dioxaborolan-2-yl)pent-3-en-1-ol (4c) Prepared according to the general procedure to give the title compound as colorless oil in $92 \%$ yield (35 mg, $Z / E>20: 1)$. ${ }^{1} \mathrm{H} \mathrm{NMR}\left(600 \mathrm{MHz}, \mathrm{CDCl}_{3}\right) \delta 7.47(\mathrm{~d}, J=8.2 \mathrm{~Hz}, 2 \mathrm{H}), 7.23$ $(\mathrm{d}, J=8.2 \mathrm{~Hz}, 2 \mathrm{H}), 6.20(\mathrm{~d}, J=9.6 \mathrm{~Hz}, 1 \mathrm{H}), 4.31(\mathrm{~d}, J=8.4 \mathrm{~Hz}, 1 \mathrm{H}), 2.81-2.87$ (m, 1H), 2.11 (brs, 1H), $1.76(\mathrm{~s}, 3 \mathrm{H}), 1.28(\mathrm{~s}, 12 \mathrm{H}), 0.77(\mathrm{~d}, J=6.6 \mathrm{~Hz}, 3 \mathrm{H}) .{ }^{13} \mathrm{C}$ NMR $(151$ $\left.\mathrm{MHz}, \mathrm{CDCl}_{3}\right) \delta 147.2,141.7,131.7,129.1,121.8,83.8,78.4,41.8,25.2,25.1,16.8,14.9$. HRMS (ESI ${ }^{+}$): $m / z$ for $\mathrm{C}_{18} \mathrm{H}_{30} \mathrm{BNO}_{3} \mathrm{Br}\left[\mathrm{M}+\mathrm{NH}_{4}\right]^{+}$calcd. 398.1502, found 398.1483. 


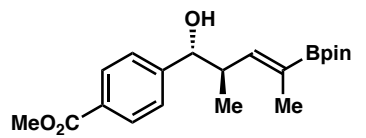

rac-Methyl-4-((1R,2R,Z)-1-hydroxy-2-methyl-4-(4,4,5,5-tetram ethyl-1,3,2-dioxaborolan-2-yl)pent-3-en-1-yl)benzoate

(4d)

Prepared according to the general procedure. The crude mixture was purified to give the title compound as white solid in 94\% yield (34 $\mathrm{mg}, Z / E>20: 1$ ). ${ }^{1} \mathrm{H}$ NMR (600 MHz, $\left.\mathrm{CDCl}_{3}\right) \delta 8.01(\mathrm{~d}, J=8.3 \mathrm{~Hz}, 2 \mathrm{H}), 7.42(\mathrm{~d}, J=8.3 \mathrm{~Hz}, 2 \mathrm{H}), 6.21$ (d, $J=9.6 \mathrm{~Hz}, 1 \mathrm{H}), 4.41$ (d, $J=8.5 \mathrm{~Hz}, 1 \mathrm{H}), 3.91$ (s, 3H), $2.84-2.91$ (m, 1H), 2.17 (brs, $1 \mathrm{H}), 1.75(\mathrm{~s}, 3 \mathrm{H}), 1.28(\mathrm{~s}, 13 \mathrm{H}), 0.78(\mathrm{~d}, J=6.7 \mathrm{~Hz}, 3 \mathrm{H}) .{ }^{13} \mathrm{C} \mathrm{NMR}\left(151 \mathrm{MHz}, \mathrm{CDCl}_{3}\right) \delta$ 167.3, 147.8, 147.1, 129.9, 129.7, 127.4, 83.8, 78.5, 52.5, 41.8, 25.2, 25.1, 16.8, 14.9. HRMS (ESI ${ }^{+}$): $m / z$ for $\mathrm{C}_{20} \mathrm{H}_{33} \mathrm{BNO}_{5}\left[\mathrm{M}+\mathrm{NH}_{4}\right]^{+}$calcd. 378.2452, found 378.2444 .

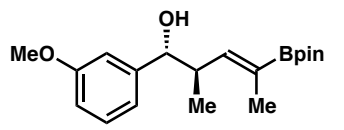

rac-(1R,2R,Z)-1-(3-Methoxyphenyl)-2-methyl-4-(4,4,5,5-tetramet hyl-1,3,2-dioxaborolan-2-yl)pent-3-en-1-ol

(4e) Prepared according to the general procedure. The crude mixture was purified to give the product as colorless oil in 93\% yield (31 mg, $Z / E>20: 1) .{ }^{1} \mathrm{H}$ NMR (600 MHz, $\left.\mathrm{CDCl}_{3}\right) \delta 7.26-7.28(\mathrm{~m}, 1 \mathrm{H}), 6.94-6.95(\mathrm{~m}, 2 \mathrm{H}), 6.84(\mathrm{~d}, J=8.0 \mathrm{~Hz}, 1 \mathrm{H}), 6.26(\mathrm{~d}, J=$ $9.5 \mathrm{~Hz}, 1 \mathrm{H}), 4.34$ (d, $J=8.6 \mathrm{~Hz}, 1 \mathrm{H}), 3.84$ (s, 3H), $2.87-2.94$ (m, 1H), 1.96 (brs, 1H), $1.79(\mathrm{~s}, 3 \mathrm{H}), 1.30(\mathrm{~s}, 12 \mathrm{H}), 0.79(\mathrm{~d}, J=6.5 \mathrm{~Hz}, 3 \mathrm{H}) .{ }^{13} \mathrm{C} \mathrm{NMR}\left(151 \mathrm{MHz}, \mathrm{CDCl}_{3}\right) \delta$ $159.8,147.9,144.3,129.5,119.9,113.5,112.7,83.7,79.0,55.6,41.7,25.2,25.1,16.9$, 14.8. HRMS (ESI $\left.{ }^{+}\right): m / z$ for $\mathrm{C}_{19} \mathrm{H}_{33} \mathrm{BNO}_{4}\left[\mathrm{M}+\mathrm{NH}_{4}\right]^{+}$calcd. 350.2503 , found 350.2487 .

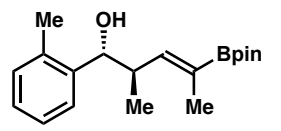

rac-(1R,2R,Z)-2-Methyl-4-(4,4,5,5-tetramethyl-1,3,2-dioxaborolan-2yl)-1-(o-tolyl)pent-3-en-1-ol (4f) Prepared according to the general procedure. The crude mixture was purified by column chromatography to give the title compound as colorless oil in $95 \%$ yield (30 mg, $Z / E>20: 1) .{ }^{1} \mathrm{H}$ NMR $\left(600 \mathrm{MHz}, \mathrm{CDCl}_{3}\right) \delta 7.45(\mathrm{~d}, J=7.5 \mathrm{~Hz}, 1 \mathrm{H}), 7.23(\mathrm{dd}, J=7.2,7.2 \mathrm{~Hz}, 1 \mathrm{H}), 7.17$ (dd, $J$ $=7.0,7.0 \mathrm{~Hz}, 1 \mathrm{H}), 7.13(\mathrm{~d}, J=7.1 \mathrm{~Hz}, 1 \mathrm{H}), 6.29(\mathrm{~d}, J=9.4 \mathrm{~Hz}, 1 \mathrm{H}), 4.71(\mathrm{~d}, J=8.8 \mathrm{~Hz}$, 1H), $2.97-3.03$ (m, 1H), 2.37 (s, 3H), 1.88 (brs, 1H), $1.76(\mathrm{~s}, 3 \mathrm{H}), 1.28$ (s, 12H), 0.79 (d, $J=6.5 \mathrm{~Hz}, 3 \mathrm{H}) .{ }^{13} \mathrm{C} \mathrm{NMR}\left(151 \mathrm{MHz}, \mathrm{CDCl}_{3}\right) \delta 148.2,140.8,136.0,130.6,127.6,126.9$, 126.6, 83.7, 74.4, 41.7, 25.2, 25.1, 20.0, 16.6, 14.8. HRMS $\left(\mathrm{ESI}^{+}\right): \mathrm{m} / z$ for $\mathrm{C}_{19} \mathrm{H}_{33} \mathrm{BNO}_{3}$ $\left[\mathrm{M}+\mathrm{NH}_{4}\right]^{+}$calcd. 334.2553 , found 334.2538 .

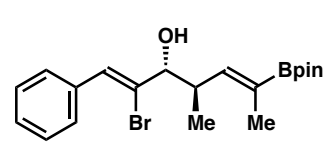

rac-(1Z,3R,4R,5Z)-2-Bromo-4-methyl-1-phenyl-6-(4,4,5,5-tetram ethyl-1,3,2-dioxaborolan-2-yl)hepta-1,5-dien-3-ol (4g) Prepared according to the general procedure. The crude mixture was purified by flash column chromatography to give the title compound as colorless oil in $86 \%$ yield $(35 \mathrm{mg}, Z / E>20: 1) .{ }^{1} \mathrm{H}$ NMR $\left(600 \mathrm{MHz}, \mathrm{CDCl}_{3}\right) \delta 7.62(\mathrm{~d}, J=5.4 \mathrm{~Hz}, 2 \mathrm{H}), 7.35-7.38$ 
$(\mathrm{m}, 2 \mathrm{H}), 7.31-7.33(\mathrm{~m}, 1 \mathrm{H}), 7.02(\mathrm{~s}, 1 \mathrm{H}), 6.24(\mathrm{~d}, J=9.3 \mathrm{~Hz}, 1 \mathrm{H}), 3.96(\mathrm{~d}, J=8.2 \mathrm{~Hz}$, $1 \mathrm{H}), 3.08-3.12$ (m, 1H), 2.06 (brs, 1H), 1.80 (s, 3H), $1.28(\mathrm{~s}, 12 \mathrm{H}), 0.97$ (d, J=6.2 Hz, $3 \mathrm{H}) .{ }^{13} \mathrm{C} \mathrm{NMR}\left(151 \mathrm{MHz}, \mathrm{CDCl}_{3}\right) \delta 146.3,135.4,130.8,129.5,128.8,128.53,128.48$, 83.7, 82.1, 38.3, 25.22, 25.17, 17.1, 14.9. HRMS $\left(\mathrm{ESI}^{+}\right): m / z$ for $\mathrm{C}_{20} \mathrm{H}_{32} \mathrm{BNO}_{3} \mathrm{Br}$ $\left[\mathrm{M}+\mathrm{NH}_{4}\right]^{+}$calcd. 424.1659 , found 424.1678 .

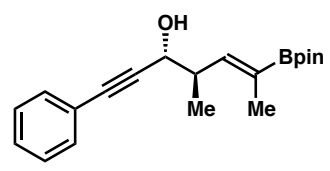

rac-(3R,4R,Z)-4-Methyl-1-phenyl-6-(4,4,5,5-tetramethyl-1,3,2-di oxaborolan-2-yl)hept-5-en-1-yn-3-ol (4h) Prepared according to the general procedure. The crude mixture was purified by flash column chromatography to give the title compound as yellow oil in $80 \%$ yield $(26 \mathrm{mg}$, $Z / E>20: 1) .{ }^{1} \mathrm{H}$ NMR $\left(600 \mathrm{MHz}, \mathrm{CDCl}_{3}\right) \delta 7.44-7.45(\mathrm{~m}, 2 \mathrm{H}), 7.31-7.32(\mathrm{~m}, 3 \mathrm{H})$, $6.17(\mathrm{~d}, J=9.5 \mathrm{~Hz}, 1 \mathrm{H}), 4.40$ (d, $J=7.5 \mathrm{~Hz}, 1 \mathrm{H}), 2.95-3.01(\mathrm{~m}, 1 \mathrm{H}), 1.98$ (brs, $1 \mathrm{H})$, $1.78(\mathrm{~s}, 3 \mathrm{H}), 1.27(\mathrm{~s}, 12 \mathrm{H}), 1.16(\mathrm{~d}, J=6.6 \mathrm{~Hz}, 3 \mathrm{H}) .{ }^{13} \mathrm{C} \mathrm{NMR}\left(151 \mathrm{MHz}, \mathrm{CDCl}_{3}\right) \delta$ $146.4,132.1,128.7,128.6,122.9,88.8,86.0,83.7,67.3,40.5,25.2,25.1,16.5,14.9$. HRMS (ESI ${ }^{+}$): $m / z$ for $\mathrm{C}_{20} \mathrm{H}_{31} \mathrm{BNO}_{3}\left[\mathrm{M}+\mathrm{NH}_{4}\right]^{+}$calcd. 344.2397, found 344.2400.

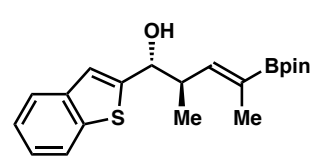

rac-(1R,2R,Z)-1-(Benzo $[b]$ thiophen-2-yl)-2-methyl-4-(4,4,5,5-tetra methyl-1,3,2-dioxaborolan-2-yl)pent-3-en-1-ol (4i) Prepared with the general procedure to give the title compound as colorless oil in $89 \%$ yield (32 mg, $Z / E=17: 1) .{ }^{1} \mathrm{H} \mathrm{NMR}\left(600 \mathrm{MHz}, \mathrm{CDCl}_{3}\right) \delta 7.82(\mathrm{~d}, J=7.5 \mathrm{~Hz}, 1 \mathrm{H}), 7.72$ $(\mathrm{d}, J=7.3 \mathrm{~Hz}, 1 \mathrm{H}), 7.29-7.35(\mathrm{~m}, 2 \mathrm{H}), 7.22(\mathrm{~s}, 1 \mathrm{H}), 6.26(\mathrm{~d}, J=9.1 \mathrm{~Hz}, 1 \mathrm{H}), 4.71(\mathrm{~d}, J$ $=8.3 \mathrm{~Hz}, 1 \mathrm{H}), 2.98-3.04(\mathrm{~m}, 1 \mathrm{H}), 2.28(\mathrm{brs}, 1 \mathrm{H}), 1.82(\mathrm{~s}, 3 \mathrm{H}), 1.29(\mathrm{~s}, 12 \mathrm{H}), 0.91(\mathrm{~d}, J$ $=5.5 \mathrm{~Hz}, 3 \mathrm{H}$ ). ${ }^{13} \mathrm{C} \mathrm{NMR}\left(151 \mathrm{MHz}, \mathrm{CDCl}_{3}\right) \delta 147.1,146.9,139.9,139.5,124.5$ (two overlapping carbon signals), 123.7, 122.8, 122.2, 83.8, 75.4, 42.0, 25.2, 25.1, 17.0, 15.0. HRMS (ESI ${ }^{+}$): $m / z$ for $\mathrm{C}_{20} \mathrm{H}_{27} \mathrm{BO}_{3} \mathrm{SNa}[\mathrm{M}+\mathrm{Na}]^{+}$calcd. 381.1672, found 381.1666.

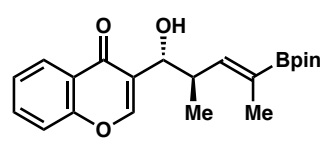

rac-3-((1R,2R,Z)-1-Hydroxy-2-methyl-4-(4,4,5,5-tetramethyl-1,3, 2-dioxaborolan-2-yl)pent-3-en-1-yl)-4H-chromen-4-one(4j)

Prepared according to the general procedure. The crude mixture was purified to give the title compound as white solid in $86 \%$ yield $(32 \mathrm{mg}, Z / E>20: 1) .{ }^{1} \mathrm{H}$ NMR $\left(600 \mathrm{MHz}, \mathrm{CDCl}_{3}\right) \delta 8.21(\mathrm{~d}, J=7.9 \mathrm{~Hz}, 1 \mathrm{H}), 7.86(\mathrm{~s}, 1 \mathrm{H}), 7.68(\mathrm{dd}, J=8.1,7.3$ $\mathrm{Hz}, 1 \mathrm{H}), 7.47$ (d, $J=8.4 \mathrm{~Hz}, 1 \mathrm{H}), 7.42(\mathrm{dd}, J=7.4,7.4 \mathrm{~Hz}, 1 \mathrm{H}), 6.28$ (d, $J=9.6 \mathrm{~Hz}, 1 \mathrm{H})$, $4.46(\mathrm{~d}, J=5.8 \mathrm{~Hz}, 1 \mathrm{H}), 3.30$ (brs, $1 \mathrm{H}), 3.19-3.24(\mathrm{~m}, 1 \mathrm{H}), 1.71(\mathrm{~s}, 3 \mathrm{H}), 1.26(\mathrm{~s}, 12 \mathrm{H})$, $0.97(\mathrm{~d}, J=6.1 \mathrm{~Hz}, 3 \mathrm{H}) .{ }^{13} \mathrm{C} \mathrm{NMR}\left(151 \mathrm{MHz}, \mathrm{CDCl}_{3}\right) \delta 178.3,156.5,153.6,147.4$, $134.2,126.1,125.6,124.5,124.3,118.5,83.6,73.7,38.8,25.3,25.1,17.7,14.8$. HRMS $\left(\mathrm{ESI}^{+}\right): \mathrm{m} / z$ for $\mathrm{C}_{21} \mathrm{H}_{27} \mathrm{BO}_{5} \mathrm{Na}[\mathrm{M}+\mathrm{Na}]^{+}$calcd. 393.1849, found 393.1831 . 


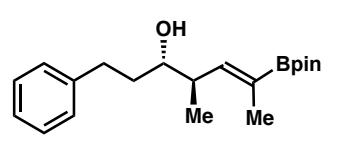

rac-(3S,4R,Z)-4-Methyl-1-phenyl-6-(4,4,5,5-tetramethyl-1,3,2-dio xaborolan-2-yl)hept-5-en-3-ol (4k) Prepared according to the general procedure $\left(-20^{\circ} \mathrm{C}\right)$ to give the title compound as white solid in $70 \%$ yield $(23 \mathrm{mg}, \mathrm{Z} / E>20: 1) .{ }^{1} \mathrm{H}$ NMR $\left(600 \mathrm{MHz}, \mathrm{CDCl}_{3}\right) \delta 7.27-7.30(\mathrm{~m}, 2 \mathrm{H})$, $7.18-7.22$ (m, 3H), 6.14 (d, $J=9.6 \mathrm{~Hz}, 1 \mathrm{H}), 3.41$ (app. dd, $J=6.4,6.1 \mathrm{~Hz}, 1 \mathrm{H}), 2.83-$ $2.88(\mathrm{~m}, 1 \mathrm{H}), 2.64-2.69(\mathrm{~m}, 2 \mathrm{H}), 1.83-1.87(\mathrm{~m}, 1 \mathrm{H}), 1.73(\mathrm{~s}, 3 \mathrm{H}), 1.67-1.73(\mathrm{~m}, 1 \mathrm{H})$, $1.27(\mathrm{~s}, 12 \mathrm{H}), 0.96(\mathrm{~d}, J=6.6 \mathrm{~Hz}, 3 \mathrm{H}) .{ }^{13} \mathrm{C} \mathrm{NMR}\left(151 \mathrm{MHz}, \mathrm{CDCl}_{3}\right) \delta 147.5,142.7$, $128.8,128.7,126.1,83.6,74.8,39.6,36.2,32.4,25.2,25.1,16.7,14.8$. HRMS $\left(\mathrm{ESI}^{+}\right)$: $m / z$ for $\mathrm{C}_{20} \mathrm{H}_{35} \mathrm{BNO}_{3}\left[\mathrm{M}+\mathrm{NH}_{4}\right]^{+}$calcd. 348.2710, found 348.2703.

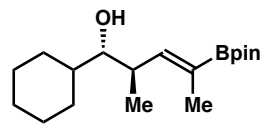

rac-(1S,2R,Z)-1-Cyclohexyl-2-methyl-4-(4,4,5,5-tetramethyl-1,3,2-di oxaborolan-2-yl)pent-3-en-1-ol (4l) Prepared according to the general procedure $\left(-20{ }^{\circ} \mathrm{C}\right)$ to give the title compound as white solid in $91 \%$ yield $(28 \mathrm{mg}, Z / E>20: 1) .{ }^{1} \mathrm{H} \mathrm{NMR}\left(600 \mathrm{MHz}, \mathrm{CDCl}_{3}\right) \delta 6.20(\mathrm{~d}, J=9.8 \mathrm{~Hz}, 1 \mathrm{H}), 3.15-$ $3.17(\mathrm{~m}, 1 \mathrm{H}), 2.75-2.81(\mathrm{~m}, 1 \mathrm{H}), 1.72-1.77(\mathrm{~m}, 3 \mathrm{H}), 1.71$ (s, 3H), 1.64 (app. d, $J=$ $11.2 \mathrm{~Hz}, 1 \mathrm{H}), 1.51-1.55(\mathrm{~m}, 2 \mathrm{H}), 1.36-1.42(\mathrm{~m}, 1 \mathrm{H}), 1.26(\mathrm{~s}, 12 \mathrm{H}), 1.09-1.26(\mathrm{~m}$, $4 \mathrm{H}), 0.94(\mathrm{~d}, J=6.6 \mathrm{~Hz}, 3 \mathrm{H}) .{ }^{13} \mathrm{C} \mathrm{NMR}\left(151 \mathrm{MHz}, \mathrm{CDCl}_{3}\right) \delta 148.2,128.4,83.6,79.7$, 40.1, 36.0, 30.7, 26.9, 26.8, 26.5, 26.4, 25.2, 25.1, 17.0, 14.7. HRMS $\left(\mathrm{ESI}^{+}\right): m / z$ for $\mathrm{C}_{18} \mathrm{H}_{37} \mathrm{BNO}_{3}\left[\mathrm{M}+\mathrm{NH}_{4}\right]^{+}$calcd. 326.2866, found 326.2870.

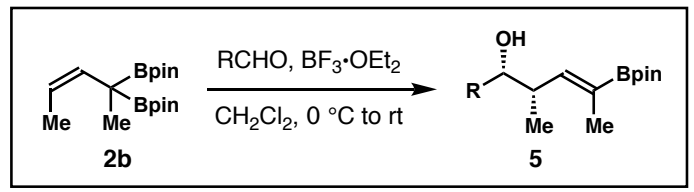

To a reaction flask containing a stirring bar were added allylboronate $\mathbf{2 b}$ (42 $\mathrm{mg}, 0.13$ mmol) and dichloromethane $(2 \mathrm{~mL})$. The mixture was cooled to $0{ }^{\circ} \mathrm{C}$ in an ice bath and stirred for $15 \mathrm{~min}$, and $\mathrm{BF}_{3} \cdot \mathrm{OEt}_{2}\left(0.5 \mathrm{M}_{\text {in }} \mathrm{CH}_{2} \mathrm{Cl}_{2}, 40 \mu \mathrm{L}, 0.02 \mathrm{mmol}\right)$ was added. After stirring for $5 \mathrm{~min}$ at $0{ }^{\circ} \mathrm{C}$, freshly distilled aldehyde $(0.1 \mathrm{mmol}$, if it is a liquid) was added slowly to the reaction mixture via a microliter syringe. The reaction mixture was allowed to warm to ambient temperature and stirred until complete consumption of the aldehyde (typically after $12 \mathrm{~h}$ ). Then saturated aqueous sodium bicarbonate solution $(1 \mathrm{~mL})$, diethyl ether $(10 \mathrm{~mL})$ and brine $(5 \mathrm{~mL})$ were added, and the resulting mixture was stirred for $3 \mathrm{~h}$. The organic layer was separated and the aqueous layer was extracted with $\mathrm{Et}_{2} \mathrm{O}$ $(3 \times 5 \mathrm{~mL})$. The combined organic extracts were dried over anhydrous sodium sulfate, filtered, and concentrated under reduced pressure. Purification of the crude product by flash column chromatography (gradient elution, 20:1 to 5:1) provided product 5. 


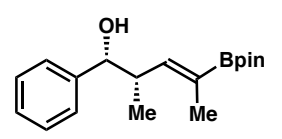

rac-(1R,2S,Z)-2-Methyl-1-phenyl-4-(4,4,5,5-tetramethyl-1,3,2-dioxab orolan-2-yl)pent-3-en-1-ol (5a) Prepared according to the general procedure. The crude mixture was purified by column chromatography to give the title compound as colorless oil in $76 \%$ yield $(23 \mathrm{mg}, Z / E=10: 1) .{ }^{1} \mathrm{H} \mathrm{NMR}$ $\left(400 \mathrm{MHz}, \mathrm{CDCl}_{3}\right) \delta 7.28-7.31(\mathrm{~m}, 4 \mathrm{H}), 7.22-7.26(\mathrm{~m}, 1 \mathrm{H}), 6.18(\mathrm{~d}, J=9.1 \mathrm{~Hz}, 1 \mathrm{H})$, $4.61(\mathrm{dd}, J=6.0,4.0 \mathrm{~Hz}, 1 \mathrm{H}), 2.88-2.97(\mathrm{~m}, 1 \mathrm{H}), 1.91(\mathrm{~d}, J=4.0 \mathrm{~Hz}, 1 \mathrm{H}), 1.55(\mathrm{~d}, J=$ $0.7 \mathrm{~Hz}, 3 \mathrm{H}), 1.24(\mathrm{~s}, 6 \mathrm{H}), 1.23(\mathrm{~s}, 6 \mathrm{H}), 1.02$ (d, $J=6.8 \mathrm{~Hz}, 3 \mathrm{H}) .{ }^{13} \mathrm{C}$ NMR $(101 \mathrm{MHz}$, $\left.\mathrm{CDCl}_{3}\right) \delta 147.7,143.5,128.3,127.6,126.7,83.5,77.9,40.7,25.2,25.0,15.3,14.4$. HRMS (ESI ${ }^{+}$): $m / z$ for $\mathrm{C}_{18} \mathrm{H}_{27} \mathrm{BO}_{3} \mathrm{Na}[\mathrm{M}+\mathrm{Na}]^{+}$calcd. 325.1951, found 325.1948.

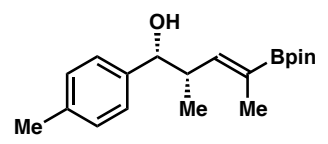

rac-(1R,2S,Z)-2-Methyl-4-(4,4,5,5-tetramethyl-1,3,2-dioxaborola n-2-yl)-1-(p-tolyl)pent-3-en-1-ol (5b) Prepared according to the general procedure. The crude mixture was purified to give the title compound as colorless oil in $82 \%$ yield $(26 \mathrm{mg}, Z / E=12: 1) .{ }^{1} \mathrm{H}$ NMR (400 $\mathrm{MHz}, \mathrm{CDCl}_{3}$ ) $\delta 7.19(\mathrm{~d}, J=7.8 \mathrm{~Hz}, 2 \mathrm{H}), 7.11(\mathrm{~d}, J=7.8 \mathrm{~Hz}, 2 \mathrm{H}), 6.18(\mathrm{~d}, J=9.5 \mathrm{~Hz}, 1 \mathrm{H}), 4.57-4.60$ $(\mathrm{m}, 1 \mathrm{H}), 2.82-2.96(\mathrm{~m}, 1 \mathrm{H}), 2.33(\mathrm{~s}, 3 \mathrm{H}), 1.85(\mathrm{~d}, J=3.6 \mathrm{~Hz}, 1 \mathrm{H}), 1.57(\mathrm{~s}, 3 \mathrm{H}), 1.24(\mathrm{~s}$, 12H), $1.01(\mathrm{~d}, J=6.7 \mathrm{~Hz}, 3 \mathrm{H}) .{ }^{13} \mathrm{C} \mathrm{NMR}\left(101 \mathrm{MHz}, \mathrm{CDCl}_{3}\right) \delta 147.8,140.5,137.1,129.0$, 126.6, 83.5, 77.7, 40.6, 25.2, 25.0, 21.5, 15.3, 14.4. HRMS $\left(\mathrm{ESI}^{+}\right): \mathrm{m} / z$ for $\mathrm{C}_{19} \mathrm{H}_{29} \mathrm{BO}_{3} \mathrm{Na}$ $[\mathrm{M}+\mathrm{Na}]^{+}$calcd. 339.2107, found 339.2116.

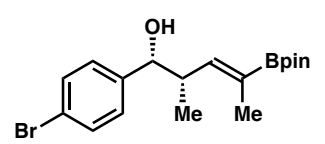

rac-(1R,2S,Z)-1-(4-Bromophenyl)-2-methyl-4-(4,4,5,5-tetramethyl -1,3,2-dioxaborolan-2-yl)pent-3-en-1-ol (5c) Prepared according to the general procedure. The crude mixture was purified by column chromatography to give the title compound as colorless oil in $76 \%$ yield $(29 \mathrm{mg}, Z / E=$ 7:1). ${ }^{1} \mathrm{H}$ NMR (400 MHz, $\left.\mathrm{CDCl}_{3}\right) \delta 7.42(\mathrm{~d}, J=8.3 \mathrm{~Hz}, 2 \mathrm{H}), 7.18(\mathrm{~d}, J=8.2 \mathrm{~Hz}, 2 \mathrm{H})$, $6.13(\mathrm{~d}, J=9.4 \mathrm{~Hz}, 1 \mathrm{H}), 4.55-4.59(\mathrm{~m}, 1 \mathrm{H}), 2.84-2.93(\mathrm{~m}, 1 \mathrm{H}), 1.93(\mathrm{~d}, J=3.9 \mathrm{~Hz}$, $1 \mathrm{H}), 1.55(\mathrm{~s}, 3 \mathrm{H}), 1.25(\mathrm{~s}, 6 \mathrm{H}), 1.24(\mathrm{~s}, 6 \mathrm{H}), 1.00(\mathrm{~d}, J=6.7 \mathrm{~Hz}, 3 \mathrm{H}) .{ }^{13} \mathrm{C}$ NMR $(151$ $\left.\mathrm{MHz}, \mathrm{CDCl}_{3}\right) \delta 147.0,142.3,131.3,128.4,121.3,83.6,77.2,40.5,25.2,24.9,15.3,14.5$. HRMS $\left(\mathrm{ESI}^{+}\right): m / z$ for $\mathrm{C}_{18} \mathrm{H}_{26} \mathrm{BO}_{3} \mathrm{BrNa}[\mathrm{M}+\mathrm{Na}]^{+}$calcd. 403.1056, found 403.1049.

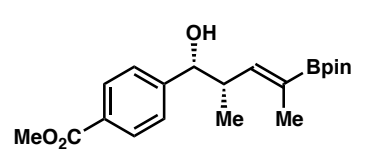

\section{rac-Methyl-4-((1R,2S,Z)-1-hydroxy-2-methyl-4-(4,4,5,5-tetrame} thyl-1,3,2-dioxaborolan-2-yl)pent-3-en-1-yl)benzoate

Prepared according to the general procedure. The crude mixture was purified by column chromatography to give the title compound as a colorless oil in $81 \%$ yield (29 mg, $Z / E=11: 1) .{ }^{1} \mathrm{H} \mathrm{NMR}\left(400 \mathrm{MHz}, \mathrm{CDCl}_{3}\right) \delta 7.97(\mathrm{~d}, J=8.3 \mathrm{~Hz}, 2 \mathrm{H})$, $7.38(\mathrm{~d}, J=8.2 \mathrm{~Hz}, 2 \mathrm{H}), 6.15(\mathrm{~d}, J=9.6 \mathrm{~Hz}, 1 \mathrm{H}), 4.65$ (dd, $J=4.9,4.4 \mathrm{~Hz}, 1 \mathrm{H}), 3.90$ (s, 
3H), $2.87-2.96(\mathrm{~m}, 1 \mathrm{H}), 2.09$ (d, $J=3.8 \mathrm{~Hz}, 1 \mathrm{H}), 1.52(\mathrm{~s}, 3 \mathrm{H}), 1.23(\mathrm{~s}, 12 \mathrm{H}), 1.01(\mathrm{~d}, J$ $=6.7 \mathrm{~Hz}, 3 \mathrm{H}) .{ }^{13} \mathrm{C} \mathrm{NMR}\left(101 \mathrm{MHz}, \mathrm{CDCl}_{3}\right) \delta 167.4,148.6,146.9,129.6,129.4,126.7$, 83.6, 77.4, 52.4, 40.7, 25.2, 25.0, 15.2, 14.4. HRMS $\left(\mathrm{ESI}^{+}\right): \mathrm{m} / \mathrm{z}$ for $\mathrm{C}_{20} \mathrm{H}_{29} \mathrm{BO}_{5} \mathrm{Na}$ $[\mathrm{M}+\mathrm{Na}]^{+}$calcd. 383.2006, found 383.1999.

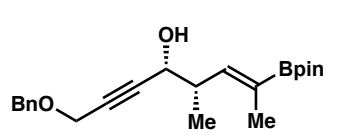

rac-(4R,5S,Z)-1-(Benzyloxy)-5-methyl-7-(4,4,5,5-tetramethyl-1,3, 2-dioxaborolan-2-yl)oct-6-en-2-yn-4-ol (5e) Prepared according to the general procedure. The crude mixture was purified to give the title compound as colorless oil in $86 \%$ yield (32 mg, $Z / E=13: 1)$. ${ }^{1} \mathrm{H}$ NMR (400 MHz, $\left.\mathrm{CDCl}_{3}\right) \delta 7.30-7.36(\mathrm{~m}, 5 \mathrm{H}), 6.30(\mathrm{~d}, J=9.2 \mathrm{~Hz}, 1 \mathrm{H}), 4.60(\mathrm{~s}, 2 \mathrm{H}), 4.35-4.38(\mathrm{~m}, 1 \mathrm{H})$, $4.22(\mathrm{~s}, 2 \mathrm{H}), 2.91-3.01(\mathrm{~m}, 1 \mathrm{H}), 1.81(\mathrm{~d}, J=7.7 \mathrm{~Hz}, 1 \mathrm{H}), 1.75(\mathrm{~s}, 3 \mathrm{H}), 1.24(\mathrm{~s}, 12 \mathrm{H})$, $1.10(\mathrm{~d}, J=6.8 \mathrm{~Hz}, 3 \mathrm{H}) .{ }^{13} \mathrm{C} \mathrm{NMR}\left(126 \mathrm{MHz}, \mathrm{CDCl}_{3}\right) \delta 145.4,137.8,128.8,128.6$,

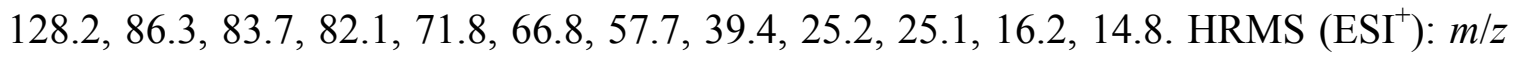
for $\mathrm{C}_{22} \mathrm{H}_{31} \mathrm{BO}_{4} \mathrm{Na}[\mathrm{M}+\mathrm{Na}]^{+}$calcd. 393.2213, found 393.2211.

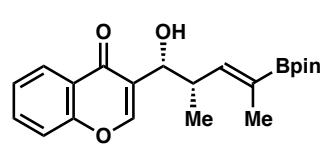

rac-3-((1R,2S,Z)-1-Hydroxy-2-methyl-4-(4,4,5,5-tetramethyl-1,3, 2-dioxaborolan-2-yl)pent-3-en-1-yl)-4H-chromen-4-one

Prepared according to the general procedure. The crude mixture was purified to give the title compound as colorless oil in $84 \%$ yield $(31 \mathrm{mg}, Z / E=10: 1) .{ }^{1} \mathrm{H}$ NMR (400 MHz, $\left.\mathrm{CDCl}_{3}\right) \delta 8.21(\mathrm{~d}, J=7.9 \mathrm{~Hz}, 1 \mathrm{H}), 7.78(\mathrm{~s}, 1 \mathrm{H}), 7.68(\mathrm{dd}, J=7.9,7.9$ $\mathrm{Hz}, 1 \mathrm{H}), 7.39-7.46(\mathrm{~m}, 2 \mathrm{H}), 6.11$ (d, $J=9.8 \mathrm{~Hz}, 1 \mathrm{H}), 4.34(\mathrm{dd}, J=7.9,7.9 \mathrm{~Hz}, 1 \mathrm{H})$, $3.61(\mathrm{~d}, J=8.4 \mathrm{~Hz}, 1 \mathrm{H}), 3.18-3.27(\mathrm{~m}, 1 \mathrm{H}), 1.56(\mathrm{~s}, 3 \mathrm{H}), 1.23(\mathrm{~s}, 12 \mathrm{H}), 1.14(\mathrm{~d}, J=6.4$ $\mathrm{Hz}, 3 \mathrm{H}) .{ }^{13} \mathrm{C} \mathrm{NMR}\left(101 \mathrm{MHz}, \mathrm{CDCl}_{3}\right) \delta 178.7,156.4,153.2,147.9,134.1,126.0,125.5$, 124.33, 124.25, 118.5, 83.6, 74.0, 38.2, 25.2, 25.0, 16.0, 14.7. HRMS $\left(\mathrm{ESI}^{+}\right): \mathrm{m} / z$ for $\mathrm{C}_{21} \mathrm{H}_{27} \mathrm{BO}_{5} \mathrm{Na}[\mathrm{M}+\mathrm{Na}]^{+}$calcd. 393.1849, found 393.1835.

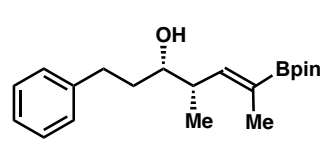

rac-(3S,4S,Z)-4-Methyl-1-phenyl-6-(4,4,5,5-tetramethyl-1,3,2-dio xaborolan-2-yl)hept-5-en-3-ol (5g) Prepared in the absence of the $\mathrm{BF}_{3} \cdot \mathrm{Et}_{2} \mathrm{O}$ catalyst at $60{ }^{\circ} \mathrm{C}$ for $12 \mathrm{~h}$. The crude mixture was purified by column chromatography to give the title compound as colorless oil in $73 \%$ yield (24 $\mathrm{mg}, Z / E=4: 1) .{ }^{1} \mathrm{H} \mathrm{NMR}\left(400 \mathrm{MHz}, \mathrm{CDCl}_{3}\right) \delta 7.26-7.29(\mathrm{~m}, 2 \mathrm{H}), 7.16-7.20(\mathrm{~m}, 3 \mathrm{H})$, $6.13(\mathrm{~d}, J=9.6 \mathrm{~Hz}, 1 \mathrm{H}), 3.46-3.51(\mathrm{~m}, 1 \mathrm{H}), 2.84(\mathrm{ddd}, J=14.6,9.6,4.8 \mathrm{~Hz}, 1 \mathrm{H}), 2.60$ $-2.72(\mathrm{~m}, 2 \mathrm{H}), 1.81-1.89(\mathrm{~m}, 1 \mathrm{H}), 1.62-1.73(\mathrm{~m}, 1 \mathrm{H}), 1.70(\mathrm{~s}, 3 \mathrm{H}), 1.42(\mathrm{~d}, J=5.0$ $\mathrm{Hz}, 1 \mathrm{H}), 1.26(\mathrm{~s}, 12 \mathrm{H}), 1.02(\mathrm{~d}, J=6.7 \mathrm{~Hz}, 3 \mathrm{H}) .{ }^{13} \mathrm{C} \mathrm{NMR}\left(101 \mathrm{MHz}, \mathrm{CDCl}_{3}\right) \delta 147.9$, $142.6,128.8,128.7,126.1,83.6,75.4,39.5,36.7,32.7,25.2,25.1,15.9,14.7$. HRMS $\left(\mathrm{ESI}^{+}\right.$): $m / z$ for $\mathrm{C}_{20} \mathrm{H}_{31} \mathrm{BO}_{3} \mathrm{Na}[\mathrm{M}+\mathrm{Na}]^{+}$calcd. 353.2264, found 353.2256. 


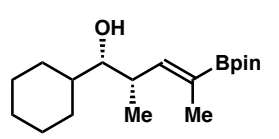

rac-(1S,2S,Z)-1-Cyclohexyl-2-methyl-4-(4,4,5,5-tetramethyl-1,3,2-di

oxaborolan-2-yl)pent-3-en-1-ol (5h) Prepared in the absence of the

$\mathrm{BF}_{3} \cdot \mathrm{Et}_{2} \mathrm{O}$ catalyst at $60{ }^{\circ} \mathrm{C}$ for $12 \mathrm{~h}$. The crude mixture was purified by column chromatography to give the title compound as colorless oil in $68 \%$ yield $(21 \mathrm{mg}$, $Z / E=3: 1) .{ }^{1} \mathrm{H}$ NMR $\left(400 \mathrm{MHz}, \mathrm{CDCl}_{3}\right) \delta 6.17(\mathrm{~d}, J=9.6 \mathrm{~Hz}, 1 \mathrm{H}), 3.23(\mathrm{dd}, J=5.9,5.5$ $\mathrm{Hz}, 1 \mathrm{H}), 2.71-2.80(\mathrm{~m}, 1 \mathrm{H}), 1.71-1.79(\mathrm{~m}, 3 \mathrm{H}), 1.70(\mathrm{~s}, 3 \mathrm{H}), 1.59-1.69(\mathrm{~m}, 3 \mathrm{H}), 1.35$ - $1.42(\mathrm{~m}, 1 \mathrm{H}), 1.27(\mathrm{~s}, 12 \mathrm{H}), 1.08-1.26(\mathrm{~m}, 4 \mathrm{H}), 1.00(\mathrm{~d}, J=6.7 \mathrm{~Hz}, 3 \mathrm{H}) .{ }^{13} \mathrm{C}$ NMR $\left(126 \mathrm{MHz}, \mathrm{CDCl}_{3}\right) \delta 149.1,83.5,79.8,41.0,35.8,30.5,27.4,26.9,26.7,26.5,25.2,25.1$, 15.2, 14.5. HRMS $\left(\mathrm{ESI}^{+}\right): \mathrm{m} / z$ for $\mathrm{C}_{18} \mathrm{H}_{37} \mathrm{BO}_{3} \mathrm{~N}\left[\mathrm{M}+\mathrm{NH}_{4}\right]^{+}$calcd. 326.2866, found 326.2870 .

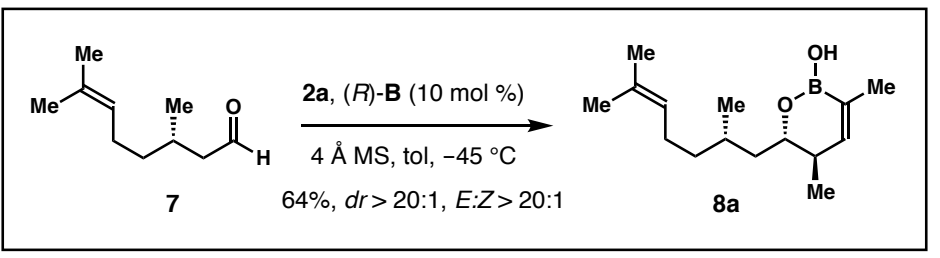

(5R,6S)-6-((S)-2,6-Dimethylhept-5-en-1-yl)-3,5-dimethyl-5,6-dihydro-2H-1,2-oxabori nin-2-ol (8a) Prepared according to the general procedure for the synthesis of $\mathbf{3}$ with 10 mol $\%$ of acid $(R)$-B as the catalyst. The crude reaction mixture was purified by column chromatography to give compound $\mathbf{8 a}$ as colorless oil in 64\% yield (16 $\mathrm{mg}, d r>20: 1$, $E: Z>20: 1) \cdot[\alpha]_{\mathrm{D}}{ }^{20}=-38.6^{\circ}\left(\mathrm{c} 0.70, \mathrm{CHCl}_{3}\right){ }^{1} \mathrm{H} \mathrm{NMR}\left(600 \mathrm{MHz}, \mathrm{CDCl}_{3}\right) \delta 6.25(\mathrm{~s}, 1 \mathrm{H})$, $5.10(\mathrm{dd}, J=6.2,6.1 \mathrm{~Hz}, 1 \mathrm{H}), 3.87(\mathrm{~s}, 1 \mathrm{H}), 3.74$ (ddd, $J=4.0,4.0,3.6 \mathrm{~Hz}, 1 \mathrm{H}), 2.17$ $2.22(\mathrm{~m}, 1 \mathrm{H}), 1.98-2.05(\mathrm{~m}, 1 \mathrm{H}), 1.88-1.94(\mathrm{~m}, 1 \mathrm{H}), 1.75(\mathrm{~s}, 3 \mathrm{H}), 1.71-1.74(\mathrm{~m}, 1 \mathrm{H})$, $1.68(\mathrm{~s}, 3 \mathrm{H}), 1.60(\mathrm{~s}, 3 \mathrm{H}), 1.39-1.48(\mathrm{~m}, 3 \mathrm{H}), 1.05-1.11(\mathrm{~m}, 1 \mathrm{H}), 1.00(\mathrm{~d}, J=7.1 \mathrm{~Hz}$, $3 \mathrm{H}), 0.93(\mathrm{~d}, J=6.7 \mathrm{~Hz}, 3 \mathrm{H}) .{ }^{13} \mathrm{C} \mathrm{NMR}\left(126 \mathrm{MHz}, \mathrm{CDCl}_{3}\right) \delta 150.1,131.4,125.3,78.0$, 43.1, 38.1, 36.4, 29.4, 26.1, 25.7, 20.9, 19.1, 18.8, 18.0. HRMS $\left(\mathrm{ESI}^{+}\right): \mathrm{m} / \mathrm{z}$ for $\mathrm{C}_{15} \mathrm{H}_{27} \mathrm{BO}_{2} \mathrm{Na}[\mathrm{M}+\mathrm{Na}]^{+}$calcd. 273.2002, found 273.2054.

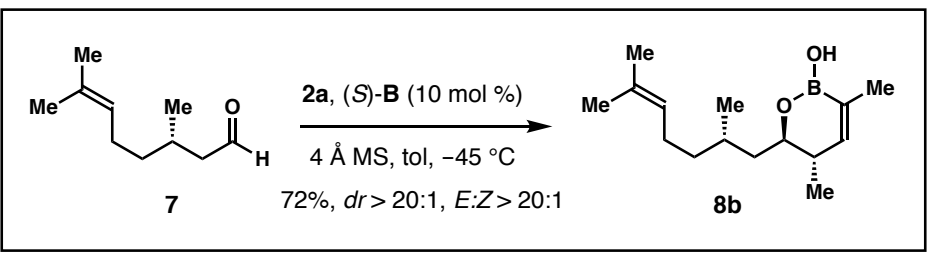

(5S,6R)-6-((S)-2,6-Dimethylhept-5-en-1-yl)-3,5-dimethyl-5,6-dihydro-2H-1,2-oxabori nin-2-ol (8b) Prepared according to the general procedure for the synthesis of $\mathbf{3}$ with 10 mol \% of acid $(S)$-B as the catalyst. The crude mixture was purified by column 
chromatography to give compound $\mathbf{8 b}$ as colorless oil in $72 \%$ yield (18 $\mathrm{mg}, d r>20: 1$, $E: Z>20: 1) .[\alpha]_{\mathrm{D}}{ }^{20}=20.8^{\circ}\left(\mathrm{c} 0.80, \mathrm{CHCl}_{3}\right) ;{ }^{1} \mathrm{H} \mathrm{NMR}\left(400 \mathrm{MHz}, \mathrm{CDCl}_{3}\right) \delta 6.25(\mathrm{~s}, 1 \mathrm{H})$, $5.11(\mathrm{dd}, J=6.5,6.0 \mathrm{~Hz}, 1 \mathrm{H}), 3.92(\mathrm{~s}, 1 \mathrm{H}), 3.73$ (app. dd, $J=9.0,8.6 \mathrm{~Hz}, 1 \mathrm{H}), 2.16$ $2.23(\mathrm{~m}, 1 \mathrm{H}), 1.91-2.06(\mathrm{~m}, 2 \mathrm{H}), 1.75(\mathrm{~s}, 3 \mathrm{H}), 1.69(\mathrm{~s}, 3 \mathrm{H}), 1.61(\mathrm{~s}, 3 \mathrm{H}), 1.55-1.58(\mathrm{~m}$, $1 \mathrm{H}), 1.15-1.34(\mathrm{~m}, 4 \mathrm{H}), 1.00(\mathrm{~d}, J=7.2 \mathrm{~Hz}, 3 \mathrm{H}), 0.89$ (d, $J=6.6 \mathrm{~Hz}, 3 \mathrm{H}) .{ }^{13} \mathrm{C}$ NMR $\left(101 \mathrm{MHz}, \mathrm{CDCl}_{3}\right) \delta 150.4,131.4,125.2,42.9,38.5,38.4,28.9,26.1,25.9,19.3,19.0$, 18.8, 18.0. HRMS $\left(\mathrm{ESI}^{+}\right): m / z$ for $\mathrm{C}_{15} \mathrm{H}_{28} \mathrm{BO}_{2}[\mathrm{M}+\mathrm{H}]^{+}$calcd. 251.2182, found 251.2194.

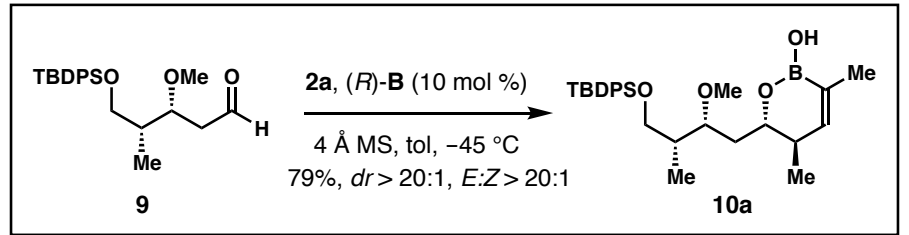

(5R,6S)-6-((2R,3R)-4-((tert-Butyldiphenylsilyl)oxy)-2-methoxy-3-methylbutyl)-3,5-di methyl-5,6-dihydro-2H-1,2-oxaborinin-2-ol (10a) Prepared from aldehyde 9 according to the general procedure for the synthesis of $\mathbf{3}$ with $10 \mathrm{~mol} \%$ of acid $(R)$-B as the catalyst. The crude mixture was purified by column chromatography to give compound 10a as colorless oil in $79 \%$ yield $(38 \mathrm{mg}, d r>20: 1, E: Z>20: 1)$. $[\alpha]_{\mathrm{D}}{ }^{20}=-21.6^{\circ}$ (c 1.80, $\mathrm{CHCl}_{3}$ ); ${ }^{1} \mathrm{H}$ NMR $\left(600 \mathrm{MHz}, \mathrm{CDCl}_{3}\right) \delta 7.66-7.68(\mathrm{~m}, 4 \mathrm{H}), 7.36-7.43(\mathrm{~m}, 6 \mathrm{H}), 6.24(\mathrm{~s}, 1 \mathrm{H})$, $3.76(\mathrm{~s}, 1 \mathrm{H}), 3.70-3.76(\mathrm{~m}, 2 \mathrm{H}), 3.64(\mathrm{ddd}, J=8.9,8.9,3.3 \mathrm{~Hz}, 1 \mathrm{H}), 3.55(\mathrm{dd}, J=9.8$, $6.1 \mathrm{~Hz}, 1 \mathrm{H}), 3.32(\mathrm{~s}, 3 \mathrm{H}), 2.23-2.29(\mathrm{~m}, 1 \mathrm{H}), 1.79-1.88(\mathrm{~m}, 2 \mathrm{H}), 1.76(\mathrm{~s}, 3 \mathrm{H}), 1.70$ (ddd, $J=13.8,8.5,3.3 \mathrm{~Hz}, 1 \mathrm{H}), 1.07$ (s, 9H), 1.01 (d, $J=7.1 \mathrm{~Hz}, 3 \mathrm{H}), 0.80$ (d, $J=7.0 \mathrm{~Hz}$, $3 \mathrm{H}) .{ }^{13} \mathrm{C} \mathrm{NMR}\left(126 \mathrm{MHz}, \mathrm{CDCl}_{3}\right) \delta 150.2,136.0,135.9,134.43,134.35,129.9,128.0$, 77.5, 77.3, 66.3, 57.8, 38.4, 38.1, 36.0, 27.2, 19.7, 18.9, 18.7, 10.4, 10.5. HRMS (ESI $\left.{ }^{+}\right)$: $m / z$ for $\mathrm{C}_{28} \mathrm{H}_{41} \mathrm{BO}_{4} \mathrm{SiNa}[\mathrm{M}+\mathrm{Na}]^{+}$calcd. 503.2765, found 503.2767.

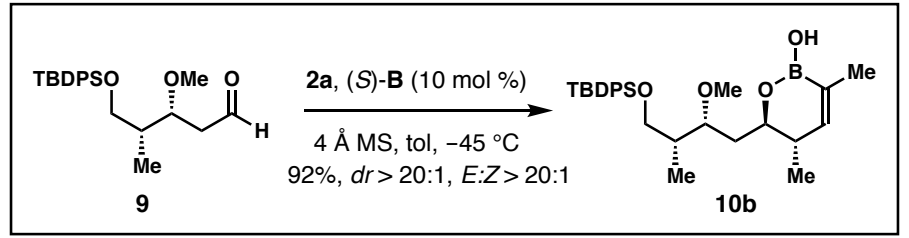

(5S,6R)-6-((2R,3R)-4-((tert-Butyldiphenylsilyl)oxy)-2-methoxy-3-methylbutyl)-3,5-di methyl-5,6-dihydro-2H-1,2-oxaborinin-2-ol (10b) Prepared from aldehyde 9 according to the general procedure for the synthesis of $\mathbf{3}$ with $10 \mathrm{~mol} \%$ of acid $(S)$-B as the catalyst. The crude mixture was purified by column chromatography to give compound $\mathbf{1 0 b}$ as colorless oil in $92 \%$ yield (44 mg, $d r>20: 1, E: Z>20: 1)$. $[\alpha]_{\mathrm{D}}{ }^{20}=+16.4^{\circ}$ (c 2.10, $\mathrm{CHCl}_{3}$ ); 
${ }^{1} \mathrm{H}$ NMR (400 MHz, $\left.\mathrm{CDCl}_{3}\right) \delta 7.66-7.69(\mathrm{~m}, 4 \mathrm{H}), 7.36-7.45(\mathrm{~m}, 6 \mathrm{H}), 6.26(\mathrm{~s}, 1 \mathrm{H})$, $3.79-3.86(\mathrm{~m}, 1 \mathrm{H}), 3.85(\mathrm{~s}, 1 \mathrm{H}), 3.68-3.73(\mathrm{~m}, 2 \mathrm{H}), 3.55(\mathrm{dd}, J=10.0,6.4 \mathrm{~Hz}, 1 \mathrm{H})$, $3.37(\mathrm{~s}, 3 \mathrm{H}), 2.15-2.23(\mathrm{~m}, 1 \mathrm{H}), 1.81-1.87(\mathrm{~m}, 1 \mathrm{H}), 1.76(\mathrm{~d}, J=1.7 \mathrm{~Hz}, 3 \mathrm{H}), 1.63$ (ddd, $J=14.0,9.8,2.6 \mathrm{~Hz}, 1 \mathrm{H}), 1.50-1.58(\mathrm{~m}, 1 \mathrm{H}), 1.07$ (s, 9H), 1.01 (d, $J=7.1 \mathrm{~Hz}$, $3 \mathrm{H}), 0.91(\mathrm{~d}, J=6.9 \mathrm{~Hz}, 3 \mathrm{H}) .{ }^{13} \mathrm{C} \mathrm{NMR}\left(126 \mathrm{MHz}, \mathrm{CDCl}_{3}\right) \delta 150.3,135.99,135.97$, $134.4,134.3,129.93$, 129.92, 128.0, 127.9, 78.2, 76.8, 66.0, 59.3, 40.1, 38.9, 38.4, 27.3, 19.7, 19.0, 18.8, 12.3. HRMS (ESI $\left.{ }^{+}\right): m / z$ for $\mathrm{C}_{28} \mathrm{H}_{41} \mathrm{BO}_{4} \mathrm{SiNa}[\mathrm{M}+\mathrm{Na}]^{+}$calcd. 503.2765, found 503.2770 .

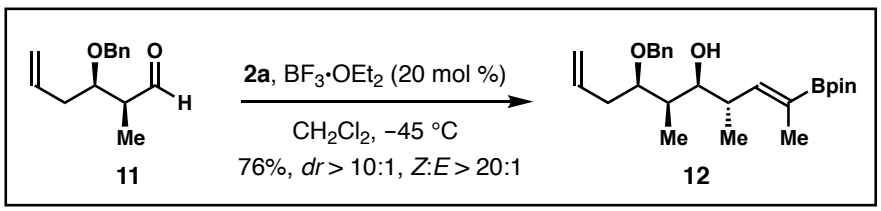

(4S,5S,6R,7R,Z)-7-(Benzyloxy)-4,6-dimethyl-2-(4,4,5,5-tetramethyl-1,3,2-dioxaborola n-2-yl)deca-2,9-dien-5-ol (14) Prepared from aldehyde 11 according to the general procedure for the synthesis of 4 with $20 \mathrm{~mol} \%$ of $\mathrm{BF}_{3} \cdot \mathrm{OEt}_{2}$ as the catalyst. The crude mixture was purified by column chromatography to give the compound $\mathbf{1 2}$ as colorless oil in $76 \%$ yield $(31 \mathrm{mg}, d r>20: 1, Z: E>20: 1)$. $[\alpha]_{\mathrm{D}}{ }^{20}=-13.5^{\circ}\left(\mathrm{c} 1.50, \mathrm{CHCl}_{3}\right) ;{ }^{1} \mathrm{H}$ NMR (400 MHz, $\left.\mathrm{CDCl}_{3}\right) \delta 7.26-7.33(\mathrm{~m}, 5 \mathrm{H}), 6.21(\mathrm{~d}, J=9.3 \mathrm{~Hz}, 1 \mathrm{H}), 5.77-5.87(\mathrm{~m}$, $1 \mathrm{H}), 5.12(\mathrm{~d}, J=16.9 \mathrm{~Hz}, 1 \mathrm{H}), 5.07$ (d, $J=10.2 \mathrm{~Hz}, 1 \mathrm{H}), 4.66(\mathrm{~d}, J=11.2 \mathrm{~Hz}, 1 \mathrm{H}), 4.45$ $(\mathrm{d}, J=11.4 \mathrm{~Hz}, 1 \mathrm{H}), 3.53-3.60(\mathrm{~m}, 2 \mathrm{H}), 2.68-2.78(\mathrm{~m}, 1 \mathrm{H}), 2.37-2.53(\mathrm{~m}, 3 \mathrm{H}), 1.83$ - $1.89(\mathrm{~m}, 1 \mathrm{H}), 1.72(\mathrm{~s}, 3 \mathrm{H}), 1.26(\mathrm{~s}, 12 \mathrm{H}), 1.01$ (d, $J=6.9 \mathrm{~Hz}, 3 \mathrm{H}), 0.86(\mathrm{~d}, J=6.7 \mathrm{~Hz}$, $3 \mathrm{H}) .{ }^{13} \mathrm{C} \mathrm{NMR}\left(101 \mathrm{MHz}, \mathrm{CDCl}_{3}\right) \delta 149.1,138.7,135.0,128.7,128.1,127.9,117.6,83.5$, 83.2, 77.0, 71.9, 37.3, 37.0, 35.6, 25.2, 16.6, 14.7, 7.4. HRMS $\left(\mathrm{ESI}^{+}\right): \mathrm{m} / z$ for $\mathrm{C}_{25} \mathrm{H}_{39} \mathrm{BO}_{4} \mathrm{Na}[\mathrm{M}+\mathrm{Na}]^{+}$calcd. 437.2839, found 437.2827.

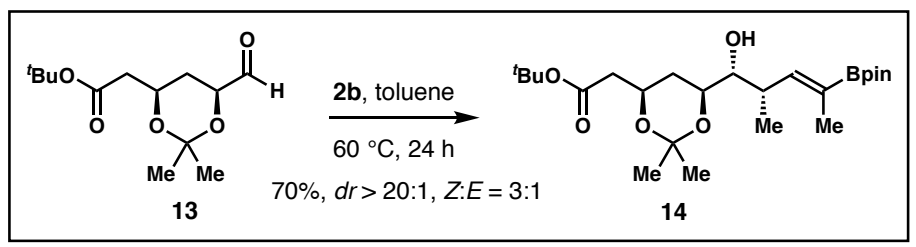

tert-Butyl-2-((4R,6S)-6-((1R,2S,Z)-1-hydroxy-2-methyl-4-(4,4,5,5-tetramethyl-1,3,2-d ioxaborolan-2-yl)pent-3-en-1-yl)-2,2-dimethyl-1,3-dioxan-4-yl)acetate (14) Prepared from aldehyde $\mathbf{1 3}$ in the absence of the $\mathrm{BF}_{3} \cdot \mathrm{Et}_{2} \mathrm{O}$ catalyst at $60{ }^{\circ} \mathrm{C}$ for $24 \mathrm{~h}$. The crude mixture was purified by column chromatography to give compound $\mathbf{1 4}$ as colorless oil in $70 \%$ yield $(32 \mathrm{mg}, d r>10: 1, Z: E=3: 1) .[\alpha]_{\mathrm{D}}{ }^{20}=-37.3^{\circ}\left(\mathrm{c} 1.50, \mathrm{CHCl}_{3}\right) ;{ }^{1} \mathrm{H} \mathrm{NMR}(600$ 
$\left.\mathrm{MHz}, \mathrm{CDCl}_{3}\right) \delta 6.11(\mathrm{~d}, J=9.9 \mathrm{~Hz}, 1 \mathrm{H}), 4.20-4.24(\mathrm{~m}, 1 \mathrm{H}), 3.83(\mathrm{ddd}, J=11.6,3.1$, $3.1 \mathrm{~Hz}, 1 \mathrm{H}), 3.50(\mathrm{dd}, J=9.1,3.6 \mathrm{~Hz}, 1 \mathrm{H}), 2.59-2.66(\mathrm{~m}, 1 \mathrm{H}), 2.41(\mathrm{dd}, J=14.9,7.5$ $\mathrm{Hz}, 1 \mathrm{H}), 2.29$ (dd, $J=15.0,5.6 \mathrm{~Hz}, 1 \mathrm{H}), 2.24(\mathrm{~s}, 1 \mathrm{H}), 1.68$ (d, $J=1.4 \mathrm{~Hz}, 3 \mathrm{H}), 1.45-$ $1.48(\mathrm{~s}, 1 \mathrm{H}), 1.44(\mathrm{~s}, 9 \mathrm{H}), 1.42(\mathrm{~s}, 3 \mathrm{H}), 1.36(\mathrm{~s}, 3 \mathrm{H}), 1.31-1.34(\mathrm{~m}, 1 \mathrm{H}), 1.28(\mathrm{~s}, 6 \mathrm{H})$, $1.27(\mathrm{~s}, 6 \mathrm{H}), 1.06(\mathrm{~d}, J=6.6 \mathrm{~Hz}, 3 \mathrm{H}) .{ }^{13} \mathrm{C} \mathrm{NMR}\left(151 \mathrm{MHz}, \mathrm{CDCl}_{3}\right) \delta 170.6,146.8,99.0$, 83.6, 80.9, 76.7, 70.4, 66.2, 43.3, 34.6, 30.3, 29.0, 28.5, 25.2, 25.1, 20.3, 17.0, 14.5. HRMS (ESI+): $m / z$ for C24H43BO7Na [M+Na]+ calcd. 477.3000, found 477.3015.

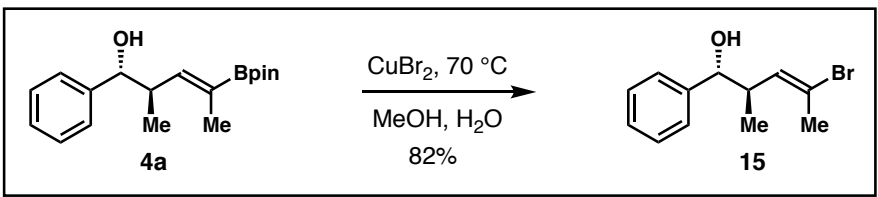

$(1 R, 2 R, E)-4-B r o m o-2-m e t h y l-1-p h e n y l p e n t-3-e n-1-o l \quad(15)$ To a reaction vial containing a Teflon-coated magnetic stirring bar was added vinylboronate 4a (30 mg, 0.1 mmol, 1.0 equiv). $\mathrm{MeOH}(0.5 \mathrm{~mL})$ and $\mathrm{H}_{2} \mathrm{O}(0.5 \mathrm{~mL})$ were added to the vial followed by addition of $\mathrm{CuBr}_{2}(89 \mathrm{mg}, 0.4 \mathrm{mmol}, 4.0$ equiv). The vial was sealed with a cap containing a PTFE-lined silicone septum and stirred at $70{ }^{\circ} \mathrm{C}$ for $12 \mathrm{~h}$. After cooling to ambient temperature, $\mathrm{H}_{2} \mathrm{O}(2 \mathrm{~mL})$ was added to the vial, and the reaction mixture was extracted with EtOAc ( 3 x $3 \mathrm{~mL}$ ). The combined organic layers were dried over anhydrous sodium sulfate, filtered, and concentrated under reduced pressure. Purification of the crude product was performed by column chromatography (hexane/ethyl acetate) to give product 15 as colorless oil in $82 \%$ yield $(21 \mathrm{mg}) .{ }^{1} \mathrm{H} \mathrm{NMR}\left(600 \mathrm{MHz}, \mathrm{CDCl}_{3}\right) \delta 7.28$ - $7.36(\mathrm{~m}, 5 \mathrm{H}), 5.81(\mathrm{dq}, J=10.0,0.8 \mathrm{~Hz}, 1 \mathrm{H}), 4.41(\mathrm{~d}, J=7.3 \mathrm{~Hz}, 1 \mathrm{H}), 2.62-2.68$ (m, $1 \mathrm{H}), 2.16(\mathrm{~d}, J=1.0 \mathrm{~Hz}, 3 \mathrm{H}), 2.01$ (brs, $1 \mathrm{H}), 0.88$ (d, $J=6.8 \mathrm{~Hz}, 3 \mathrm{H}) .{ }^{13} \mathrm{C}$ NMR $(151$ $\left.\mathrm{MHz}_{\mathrm{CDCl}}\right) \delta 142.5,134.3,128.7,128.2,127.0,121.8,78.7,43.2,24.0,17.3$.

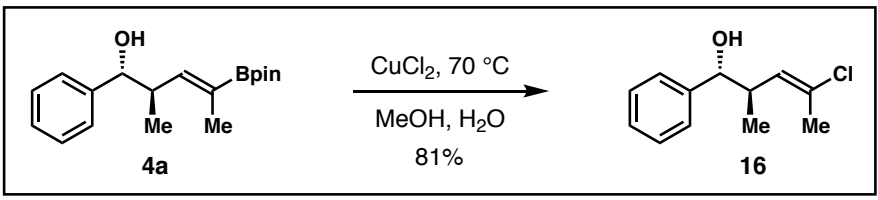

$(1 R, 2 R, E)-4-C h l o r o-2-m e t h y l-1-p h e n y l p e n t-3-e n-1-o l \quad(16)$ To a reaction vial containing a Teflon-coated magnetic stirring bar was added vinylboronate 4a (30 mg, 0.1 mmol, 1.0 equiv). $\mathrm{MeOH}(0.5 \mathrm{~mL})$ and $\mathrm{H}_{2} \mathrm{O}(0.5 \mathrm{~mL})$ were added to the vial followed by addition of $\mathrm{CuCl}_{2}$ (53 mg, $0.4 \mathrm{mmol}, 4.0$ equiv). The vial was sealed with a cap containing a PTFE-lined silicone septum and stirred at $70{ }^{\circ} \mathrm{C}$ for $12 \mathrm{~h}$. After cooling to ambient temperature, $\mathrm{H}_{2} \mathrm{O}(2 \mathrm{~mL})$ was added to the vial, and the reaction mixture was 
extracted with EtOAc ( 3 x $3 \mathrm{~mL}$ ). The combined organic layers were dried over anhydrous sodium sulfate, filtered, and concentrated under reduced pressure. Purification of the crude product was performed by column chromatography (hexane/ethyl acetate) to give product 16 as colorless oil in $81 \%$ yield $(17 \mathrm{mg}) .{ }^{1} \mathrm{H}$ NMR $\left(600 \mathrm{MHz}, \mathrm{CDCl}_{3}\right) \delta 7.28$ - $7.36(\mathrm{~m}, 5 \mathrm{H}), 5.54-5.56(\mathrm{~m}, 1 \mathrm{H}), 4.41(\mathrm{~d}, J=7.3 \mathrm{~Hz}, 1 \mathrm{H}), 2.60-2.66(\mathrm{~m}, 1 \mathrm{H}), 2.00$ (s, 3H), 1.99 (brs, 1H), 0.89 (d, $J=6.9 \mathrm{~Hz}, 3 \mathrm{H}) .{ }^{13} \mathrm{C} \mathrm{NMR}\left(151 \mathrm{MHz}, \mathrm{CDCl}_{3}\right) \delta 142.6$, 131.6, 129.9, 128.7, 128.2, 127.1, 78.9, 42.5, 21.6, 17.5. HRMS (EI $\left.{ }^{+}\right): m / z$ for $\mathrm{C}_{12} \mathrm{H}_{15} \mathrm{OCl}$ $[\mathrm{M}]^{+}$calcd. 210.0811, found 210.0811 .

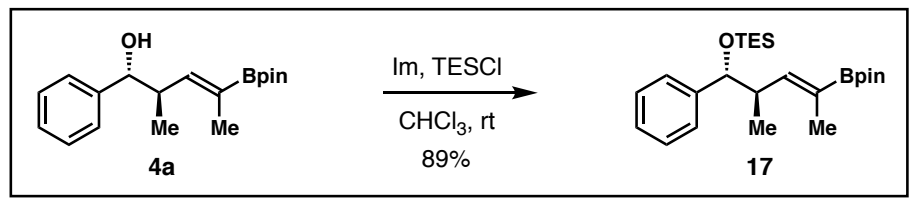

\section{Triethyl(((1R,2R,Z)-2-methyl-1-phenyl-4-(4,4,5,5-tetramethyl-1,3,2-dioxaborolan-2-}

yl)pent-3-en-1-yl)oxy)silane (17) In a reaction vial, alcohol 4a (302 mg, $1.0 \mathrm{mmol}, 1.0$ equiv), imidazole (136 mg, $2.0 \mathrm{mmol}, 2.0$ equiv) and TESCl (300 mg, $2.0 \mathrm{mmol}, 2.0$ equiv) were dissolved in $\mathrm{CHCl}_{3}(2.5 \mathrm{~mL})$. The reaction mixture was stirred at ambient temperature for $2 \mathrm{~h}$. After complete consumption of alcohol 4a, the reaction was diluted with diethyl ether $(2 \mathrm{~mL})$, and the resulting mixture was filtered through a pad of silica gel. The filtrate was concentrated under reduced pressure to provide the crude product, which was purified by flash column chromatography (hexane: ethyl acetate $=20: 1$ ) to give product 17 in $89 \%$ yield $(370 \mathrm{mg})$ as colorless oil. ${ }^{1} \mathrm{H}$ NMR $\left(600 \mathrm{MHz}, \mathrm{CDCl}_{3}\right) \delta$ $7.19-7.27$ (m, 5H), $6.18(\mathrm{dq}, J=9.5,1.3 \mathrm{~Hz}, 1 \mathrm{H}), 4.38(\mathrm{~d}, J=7.4 \mathrm{~Hz}, 1 \mathrm{H}), 2.80-2.87$ $(\mathrm{m}, 1 \mathrm{H}), 1.66(\mathrm{~d}, J=1.4 \mathrm{~Hz}, 3 \mathrm{H}), 1.27(\mathrm{~s}, 6 \mathrm{H}), 1.25(\mathrm{~s}, 6 \mathrm{H}), 0.81(\mathrm{t}, J=8.0 \mathrm{~Hz}, 9 \mathrm{H}), 0.73$ $(\mathrm{d}, J=6.8 \mathrm{~Hz}, 3 \mathrm{H}), 0.40-0.50(\mathrm{~m}, 6 \mathrm{H}) .{ }^{13} \mathrm{C} \mathrm{NMR}\left(151 \mathrm{MHz}, \mathrm{CDCl}_{3}\right) \delta 149.8,144.3$, $128.0,127.34,127.31,83.3,79.4,41.9,25.4,24.9,16.5,14.5,7.1,5.2$.

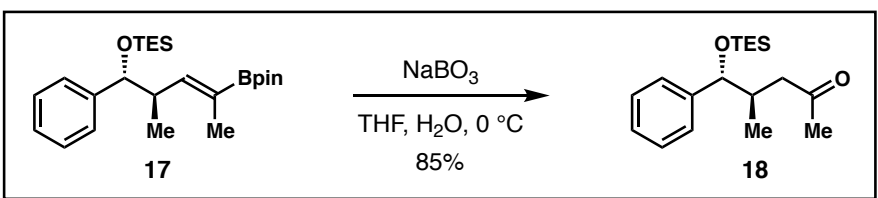

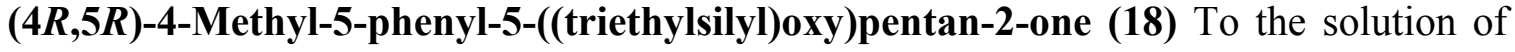
vinylboronate 17 (0.05 mmol, 1.0 equiv) in THF $(1 \mathrm{~mL})$ was added a solution of $\mathrm{NaBO}_{3} \cdot 4 \mathrm{H}_{2} \mathrm{O}$ (5 equiv) in $\mathrm{H}_{2} \mathrm{O}(2 \mathrm{~mL})$. The resulting heterogeneous mixture was allowed to stir vigorously at ambient temperature for $12 \mathrm{~h}$. The aqueous layer was extracted with diethyl ether $(3 \times 1 \mathrm{~mL})$. The combined organic extracts were dried over magnesium 
sulfate, filtered, and concentrated under reduced pressure. Purification of the crude product was performed by flash column chromatography (hexane/ethyl acetate) to give product 18 as colorless oil in 85\% yield $(13 \mathrm{mg}) .{ }^{1} \mathrm{H}$ NMR $\left(600 \mathrm{MHz}, \mathrm{CDCl}_{3}\right) \delta 7.24-$ $7.32(\mathrm{~m}, 5 \mathrm{H}), 4.44(\mathrm{~d}, J=6.2 \mathrm{~Hz}, 1 \mathrm{H}), 2.67(\mathrm{dd}, J=16.1,3.9 \mathrm{~Hz}, 1 \mathrm{H}), 2.28-2.33(\mathrm{~m}$, 1H), 2.25 (dd, $J=16.0,9.1 \mathrm{~Hz}, 1 \mathrm{H}), 2.11(\mathrm{~s}, 3 \mathrm{H}), 0.87$ (t, $J=8.0 \mathrm{~Hz}, 9 \mathrm{H}), 0.82$ (d, $J=$ $6.6 \mathrm{~Hz}, 3 \mathrm{H}), 0.45-0.54(\mathrm{~m}, 6 \mathrm{H}) .{ }^{13} \mathrm{C} \mathrm{NMR}\left(151 \mathrm{MHz}, \mathrm{CDCl}_{3}\right) \delta 209.3,144.0,128.2$, 127.5, 127.1, 79.2, 46.9, 38.3, 30.6, 17.1, 7.1, 5.1. HRMS (ESI $\left.{ }^{+}\right): m / z$ for $\mathrm{C}_{18} \mathrm{H}_{30} \mathrm{O}_{2} \mathrm{SiNa}$ $[\mathrm{M}+\mathrm{Na}]^{+}$calcd. 329.1913, found 329.1923.

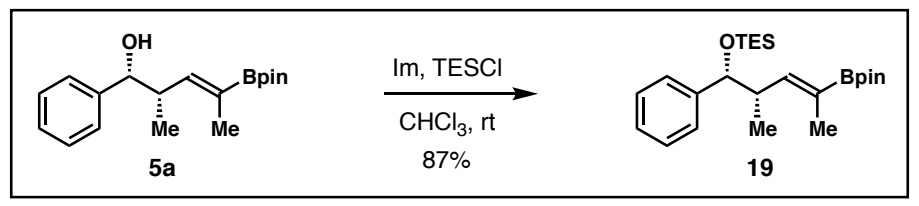

Triethyl(((1R,2S,Z)-2-methyl-1-phenyl-4-(4,4,5,5-tetramethyl-1,3,2-dioxaborolan-2-y l)pent-3-en-1-yl)oxy)silane (19) In a reaction vial, alcohol 5a $(151 \mathrm{mg}, 0.5 \mathrm{mmol}, 1.0$ equiv), imidazole (68 mg, $1.0 \mathrm{mmol}, 2.0$ equiv) and TESCl (113 mg, $0.75 \mathrm{mmol}, 1.5$ equiv) were dissolved in $\mathrm{CHCl}_{3}(2.0 \mathrm{~mL})$. The reaction mixture was stirred at ambient temperature for $1 \mathrm{~h}$. After complete consumption of alcohol 5a, the reaction was diluted with diethyl ether $(2 \mathrm{~mL})$, and the resulting mixture was filtered through a pad of silica gel. The filtrate was concentrated under reduced pressure and the crude product was purified by flash column chromatography (hexane: ethyl acetate $=20: 1$ ) to give product 19 in $87 \%$ yield $(182 \mathrm{mg})$ as colorless oil. ${ }^{1} \mathrm{H}$ NMR $\left(600 \mathrm{MHz}, \mathrm{CDCl}_{3}\right) \delta 7.21-7.26(\mathrm{~m}$, 4H), $7.15(\mathrm{dd}, J=7.0,7.0 \mathrm{~Hz}, 1 \mathrm{H}), 6.20(\mathrm{dq}, J=9.7,1.8 \mathrm{~Hz}, 1 \mathrm{H}), 4.52(\mathrm{~d}, J=5.5 \mathrm{~Hz}$, $1 \mathrm{H}), 2.72-2.78(\mathrm{~m}, 1 \mathrm{H}), 1.48(\mathrm{~d}, J=1.8 \mathrm{~Hz}, 3 \mathrm{H}), 1.22(\mathrm{~s}, 6 \mathrm{H}), 1.21(\mathrm{~s}, 6 \mathrm{H}), 0.95(\mathrm{~d}, J=$ $6.6 \mathrm{~Hz}, 3 \mathrm{H}), 0.85(\mathrm{t}, J=8.0 \mathrm{~Hz}, 9 \mathrm{H}), 0.43-0.53(\mathrm{~m}, 6 \mathrm{H}) .{ }^{13} \mathrm{C} \mathrm{NMR}\left(151 \mathrm{MHz}, \mathrm{CDCl}_{3}\right) \delta$ $149.5,144.7,127.8,127.0,126.8,83.3,78.3$, 42.0, 25.3, 24.8, 14.9, 14.2, 7.2, 5.2. HRMS $\left(\mathrm{ESI}^{+}\right): \mathrm{m} / z$ for $\mathrm{C}_{24} \mathrm{H}_{41} \mathrm{O}_{3} \mathrm{SiBNa}[\mathrm{M}+\mathrm{Na}]^{+}$calcd. 439.2816, found 439.2824.

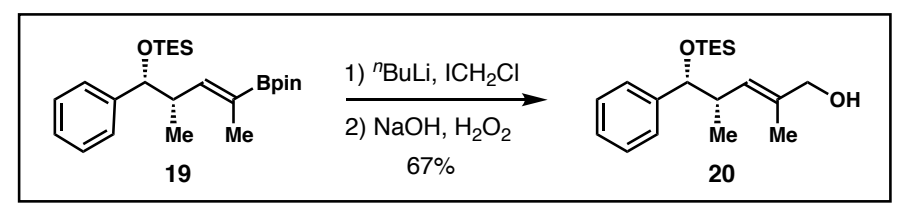

$(4 S, 5 R, E)-2,4-D i m e t h y l-5-p h e n y l-5-((t r i e t h y l s i l y l) o x y) p e n t-2-e n-1-o l \quad(20)$ To a solution of vinyl boronate 19 ( $83 \mathrm{mg}, 0.2 \mathrm{mmol}, 1.0$ equiv) in THF (1.5 mL) was added $\mathrm{ICH}_{2} \mathrm{Cl}$ (52 mg, $0.3 \mathrm{mmol}, 1.5$ equiv) under argon. The resulting colorless solution was placed in a dry ice-acetone bath and cooled to $-78{ }^{\circ} \mathrm{C}$. After stirring for $10 \mathrm{~min}$ at 
$-78^{\circ} \mathrm{C}$, n-butyl lithium (2.0 M in THF, $100 \mu \mathrm{L}, 0.2 \mathrm{mmol}, 1.0$ equiv) was added slowly to the reaction. After stirring at $-78{ }^{\circ} \mathrm{C}$ for $20 \mathrm{~min}$, the cooling bath was removed; the reaction mixture was allowed to warm to ambient temperature and stirred for additional 2 h. Then the resulting mixture was cooled to $0{ }^{\circ} \mathrm{C}$ with an ice bath. $3 \mathrm{~N} \mathrm{NaOH}$ solution (1 $\mathrm{mL})$ and $30 \% \mathrm{H}_{2} \mathrm{O}_{2}(1 \mathrm{~mL})$ were slowly added to the reaction. The resulting mixture was stirred for $3 \mathrm{~h}$ at $0{ }^{\circ} \mathrm{C}$. The organic layer was separated and the aqueous layer was extracted with diethyl ether $(3 \times 3 \mathrm{~mL})$. The combined organic extracts were dried over anhydrous sodium sulfate, filtered, and concentrated under reduced pressure. Purification of the crude product was performed by flash column chromatography to give $\mathbf{2 0}$ in $67 \%$ yield $(43 \mathrm{mg})$ as colorless oil. ${ }^{1} \mathrm{H}$ NMR $\left(600 \mathrm{MHz}, \mathrm{CDCl}_{3}\right) \delta 7.23-7.28(\mathrm{~m}, 4 \mathrm{H}), 7.17-$ $7.20(\mathrm{~m}, 1 \mathrm{H}), 5.21(\mathrm{dq}, J=9.8,1.1 \mathrm{~Hz}, 1 \mathrm{H}), 4.41(\mathrm{~d}, J=6.4 \mathrm{~Hz}, 1 \mathrm{H}), 3.87(\mathrm{~d}, J=5.5 \mathrm{~Hz}$, 2H), $2.60-2.66(\mathrm{~m}, 1 \mathrm{H}), 1.40(\mathrm{~d}, J=1.2 \mathrm{~Hz}, 3 \mathrm{H}), 1.00$ (d, $J=6.6 \mathrm{~Hz}, 3 \mathrm{H}), 0.86$ (t, $J=$ $7.9 \mathrm{~Hz}, 9 \mathrm{H}), 0.55-0.45(\mathrm{~m}, 6 \mathrm{H}) .{ }^{13} \mathrm{C} \mathrm{NMR}\left(151 \mathrm{MHz}, \mathrm{CDCl}_{3}\right) \delta$ 144.6, 135.0, 129.5, 127.9, 127.2, 127.0, 79.2, 69.4, 41.5, 16.6, 14.1, 7.1, 5.2. HRMS $\left(\mathrm{ESI}^{+}\right): \mathrm{m} / z$ for $\mathrm{C}_{19} \mathrm{H}_{32} \mathrm{O}_{2} \mathrm{SiNa}[\mathrm{M}+\mathrm{Na}]^{+}$calcd. 343.2069, found 343.2054.

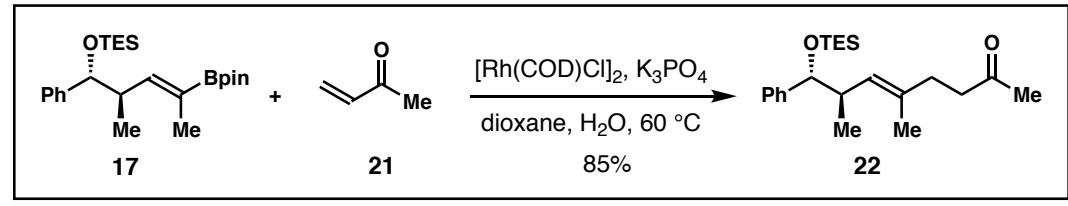

(7R,8R,E)-5,7-Dimethyl-8-phenyl-8-((triethylsilyl)oxy)oct-5-en-2-one $\quad(22)^{1}$ In an Ar-filled glove box, vinylboronate 17 (31 mg, $0.075 \mathrm{mmol}, 1.0$ equiv), methyl vinyl ketone ( $8 \mathrm{mg}, 0.11 \mathrm{mmol}, 1.5$ equiv), $\left[\mathrm{Rh}(\mathrm{COD}) \mathrm{Cl}_{2}(2 \mathrm{mg}, 4 \mu \mathrm{mol}, 5 \mathrm{~mol} \%), \mathrm{K}_{3} \mathrm{PO}_{4}\right.$ (24 mg, $0.11 \mathrm{mmol}, 1.5$ equiv), dioxane $(0.5 \mathrm{~mL})$ and a stirring bar were sequentially added into a reaction vial. The vial was sealed with rubber septum and removed from the glove box. Then water $(0.2 \mathrm{~mL})$ was added under an argon atmosphere and the mixture was stirred at $60{ }^{\circ} \mathrm{C}$ for $24 \mathrm{~h}$. After completion of the reaction, the reaction was cooled to ambient temperature and the mixture was filtered through a short pad of silica gel. The filtrate was concentrated under reduced pressure. Purification of the crude reaction product was performed by column chromatography (gradient elution with hexane/ethyl acetate, 20:1 to $10: 1)$ to give product 22 as colorless oil in $85 \%$ yield $(23 \mathrm{mg}) .{ }^{1} \mathrm{H} \mathrm{NMR}$ $\left(500 \mathrm{MHz}, \mathrm{CDCl}_{3}\right) \delta 7.21-7.29(\mathrm{~m}, 5 \mathrm{H}), 4.98(\mathrm{~d}, J=9.5 \mathrm{~Hz}, 1 \mathrm{H}), 4.49(\mathrm{~d}, J=5.4 \mathrm{~Hz}$, $1 \mathrm{H}), 2.59-2.66(\mathrm{~m}, 1 \mathrm{H}), 2.45-2.55(\mathrm{~m}, 2 \mathrm{H}), 2.24(\mathrm{t}, J=7.8 \mathrm{~Hz}, 2 \mathrm{H}), 2.16(\mathrm{~s}, 3 \mathrm{H}), 1.45$ $(\mathrm{s}, 3 \mathrm{H}), 0.88(\mathrm{t}, J=7.9 \mathrm{~Hz}, 9 \mathrm{H}), 0.85(\mathrm{~d}, J=6.6 \mathrm{~Hz}, 3 \mathrm{H}), 0.44-0.56(\mathrm{~m}, 6 \mathrm{H}) .{ }^{13} \mathrm{C} \mathrm{NMR}$ $\left(126 \mathrm{MHz}, \mathrm{CDCl}_{3}\right) \delta 209.2,144.4,134.0,128.1,127.8,127.2,127.1,79.2,42.7,41.3$, 
34.0, 30.2, 17.5, 16.6, 7.1, 5.2. HRMS $\left(\mathrm{ESI}^{+}\right): \mathrm{m} / z$ for $\mathrm{C}_{22} \mathrm{H}_{36} \mathrm{O}_{2} \mathrm{SiNa}[\mathrm{M}+\mathrm{Na}]^{+}$calcd. 383.2382, found 383.2365 .

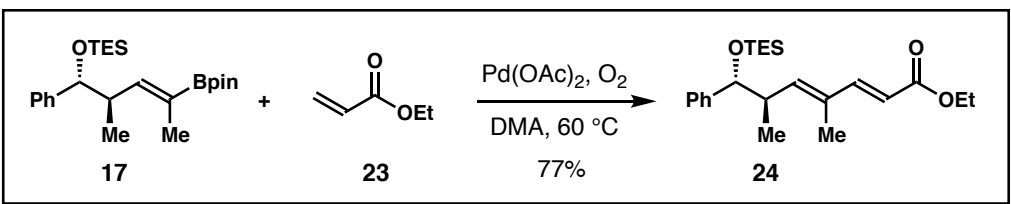

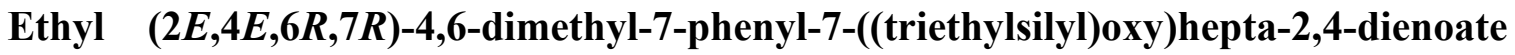
(24) To a solution of vinyl boronate 17 (42 $\mathrm{mg}, 0.10 \mathrm{mmol}, 1.0$ equiv) in $N, N$-dimethylacetamide $(0.5 \mathrm{~mL})$ was added $\mathrm{Pd}(\mathrm{OAc})_{2}(2 \mathrm{mg}, 0.01 \mathrm{mmol}, 10 \mathrm{~mol} \%)$ and ethyl acrylate (32 $\mu \mathrm{L}, 0.30 \mathrm{mmol}, 3.0$ equiv). The reaction flask was fitted with an oxygen balloon and the reaction mixture was stirred at $60{ }^{\circ} \mathrm{C}$ for $18 \mathrm{~h}$. Then diethyl ether $(20 \mathrm{~mL})$ was added to the reaction flask, and the resulting solution was washed with water $(2 \times 10 \mathrm{~mL})$. The organic layer was dried over anhydrous $\mathrm{Na}_{2} \mathrm{SO}_{4}$, filtered, and the filtrate was concentrated under reduced pressure. Purification of the crude product was performed by flash column chromatography (gradient elution with hexane and ethyl acetate) to give product 24 in $77 \%$ yield $(30 \mathrm{mg})$ as colorless oil. ${ }^{1} \mathrm{H}$ NMR (400 MHz, $\left.\mathrm{CDCl}_{3}\right) \delta 7.31(\mathrm{~d}, J=15.7 \mathrm{~Hz}, 1 \mathrm{H}), 7.19-7.26(\mathrm{~m}, 5 \mathrm{H}), 5.79(\mathrm{~d}, J=9.9 \mathrm{~Hz}, 1 \mathrm{H}), 5.74(\mathrm{~d}$, $J=15.7 \mathrm{~Hz}, 1 \mathrm{H}), 4.52$ (d, $J=5.4 \mathrm{~Hz}, 1 \mathrm{H}), 4.21$ (q, $J=7.0 \mathrm{~Hz}, 2 \mathrm{H}), 2.74-2.83(\mathrm{~m}, 1 \mathrm{H})$, $1.58(\mathrm{~s}, 3 \mathrm{H}), 1.30(\mathrm{t}, J=7.1 \mathrm{~Hz}, 3 \mathrm{H}), 0.92$ (d, $J=6.8 \mathrm{~Hz}, 3 \mathrm{H}), 0.84$ (t, $J=8.0 \mathrm{~Hz}, 9 \mathrm{H})$, 0.47 (q, $J=8.1 \mathrm{~Hz}, 6 \mathrm{H}) .{ }^{13} \mathrm{C} \mathrm{NMR}\left(151 \mathrm{MHz}, \mathrm{CDCl}_{3}\right) \delta 168.0,150.2,144.7,144.0$, $133.3,128.1,127.4,126.9,115.9,79.1,60.5,42.5,17.3,14.7,12.7,7.1,5.1$. HRMS $\left(\mathrm{ESI}^{+}\right): \mathrm{m} / z$ for $\mathrm{C}_{23} \mathrm{H}_{36} \mathrm{O}_{3} \mathrm{SiNa}[\mathrm{M}+\mathrm{Na}]^{+}$calcd. 411.2331, found 411.2327.

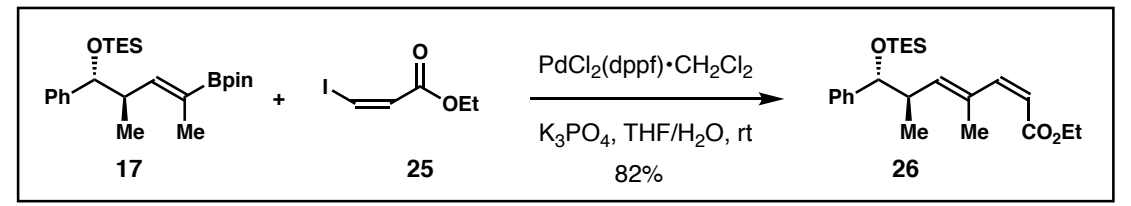

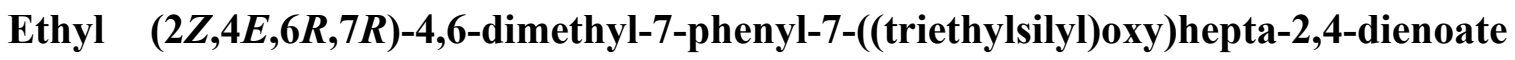
(26) In an Ar-filled glove box, $\mathrm{PdCl}_{2}(\mathrm{dppf}) \cdot \mathrm{CH}_{2} \mathrm{Cl}_{2}(8 \mathrm{mg}, 0.01 \mathrm{mmol}, 10 \mathrm{~mol} \%), \mathrm{K}_{3} \mathrm{PO}_{4}$ (55 mg, $0.26 \mathrm{mmol}, 2.6$ equiv), THF ( $0.5 \mathrm{~mL}$ ), vinylboronate 17 (42 mg, $0.1 \mathrm{mmol}, 1.0$ equiv), and a Teflon-coated magnetic stirring bar were sequentially added into a 1-dram vial. The vial was sealed with a rubber septum and removed from glove box. Then vinyl iodide 25 (29 mg, $0.13 \mathrm{mmol}, 1.3$ equiv) and $50 \mu \mathrm{L} \mathrm{H}_{2} \mathrm{O}$ were added to the mixture under argon. The reaction was kept stirring at ambient temperature for $48 \mathrm{~h}$. After complete consumption of boronate $17, \mathrm{Et}_{2} \mathrm{O}(2 \mathrm{~mL})$ was added and the resulting mixture was 
filtered through a short pad of Celite. Brine $(5 \mathrm{~mL})$ and $\mathrm{Et}_{2} \mathrm{O}(1 \mathrm{~mL})$ were added to the filtrate, the organic layer was separated, and the aqueous layer was extracted with $\mathrm{Et}_{2} \mathrm{O}$ (3 $\mathrm{x} 1 \mathrm{~mL}$ ). The combined organic layers were dried over anhydrous magnesium sulfate, filtered, and concentrated under reduced pressure. Purification of the crude product was performed by flash chromatography to provide product 26 in $82 \%$ yield $(32 \mathrm{mg}$ ) as colorless oil. ${ }^{1} \mathrm{H}$ NMR $\left(600 \mathrm{MHz}, \mathrm{CDCl}_{3}\right) \delta 7.28-7.32(\mathrm{~m}, 4 \mathrm{H}), 7.24-7.26(\mathrm{~m}, 1 \mathrm{H})$, $6.39(\mathrm{dd}, J=12.7,0.7 \mathrm{~Hz}, 1 \mathrm{H}), 5.63(\mathrm{~d}, J=12.6 \mathrm{~Hz}, 1 \mathrm{H}), 5.59(\mathrm{~d}, J=9.7 \mathrm{~Hz}, 1 \mathrm{H}), 4.54$ $(\mathrm{d}, J=5.8 \mathrm{~Hz}, 1 \mathrm{H}), 4.16-4.24(\mathrm{~m}, 2 \mathrm{H}), 2.76-2.82(\mathrm{~m}, 1 \mathrm{H}), 1.76(\mathrm{~d}, J=1.1 \mathrm{~Hz}, 3 \mathrm{H})$, $1.32(\mathrm{t}, J=7.1 \mathrm{~Hz}, 3 \mathrm{H}), 0.91(\mathrm{~d}, J=6.8 \mathrm{~Hz}, 3 \mathrm{H}), 0.88(\mathrm{t}, J=8.0 \mathrm{~Hz}, 9 \mathrm{H}), 0.48-0.55(\mathrm{~m}$, $6 \mathrm{H}) .{ }^{13} \mathrm{C} \mathrm{NMR}\left(151 \mathrm{MHz}, \mathrm{CDCl}_{3}\right) \delta 167.4,145.7,144.0,141.7,133.4,128.0,127.4$, 127.1, 116.9, 79.0, 60.5, 42.0, 16.8, 15.4, 14.6, 7.1, 5.2. HRMS $\left(\mathrm{ESI}^{+}\right): \mathrm{m} / z$ for $\mathrm{C}_{23} \mathrm{H}_{36} \mathrm{O}_{3} \mathrm{SiNa}[\mathrm{M}+\mathrm{Na}]^{+}$calcd. 411.2331, found 411.2327 .

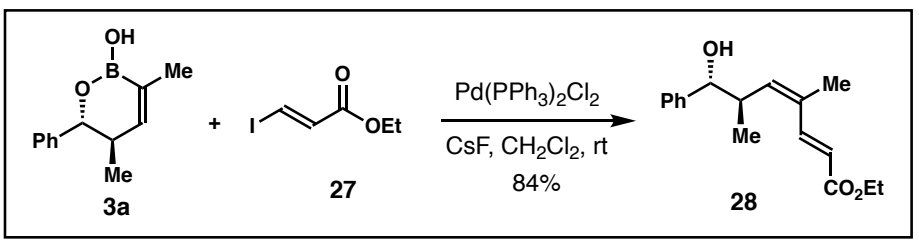

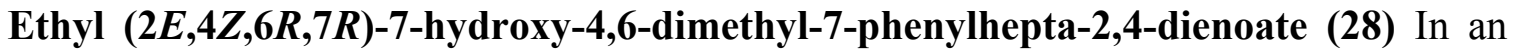
Ar-filled glove box, $\mathrm{Pd}\left(\mathrm{PPh}_{3}\right)_{2} \mathrm{Cl}_{2}(4 \mathrm{mg}, 5.0 \mu \mathrm{mol}, 5 \mathrm{~mol} \%)$ and $\mathrm{CsF}$ (30 mg, $0.2 \mathrm{mmol}$, 2.0 equiv) and a Teflon-coated magnetic stirring bar were sequentially added into a 1-dram vial. The vial was sealed with rubber septum and removed from the glove box. Then vinyl iodide 27 ( $23 \mathrm{mg}, 0.1 \mathrm{mmol}, 1.0$ equiv), and vinyl boron 3a (20 mg, $0.1 \mathrm{mmol}$, 1.0 equiv) in $\mathrm{CH}_{2} \mathrm{Cl}_{2}(0.5 \mathrm{~mL})$ was added to the vial under argon. The mixture was stirred for $24 \mathrm{~h}$ at ambient temperature. After completion of the reaction, the mixture was filtered through a pad of silica gel. The filtrate was concentrated under reduced pressure and the crude product was purified by preparative thin-layer chromatography (hexane/ethyl acetate $=30: 1$ to $10: 1$ ) to give product 28 in $84 \%$ yield (23 $\mathrm{mg}$ ) as colorless oil. ${ }^{1} \mathrm{H}$ NMR $\left(500 \mathrm{MHz}, \mathrm{CDCl}_{3}\right) \delta 7.70(\mathrm{~d}, J=15.6 \mathrm{~Hz}, 1 \mathrm{H}), 7.32-7.36(\mathrm{~m}, 4 \mathrm{H}), 7.27-$ $7.30(\mathrm{~m}, 1 \mathrm{H}), 5.91(\mathrm{~d}, J=15.6 \mathrm{~Hz}, 1 \mathrm{H}), 5.68(\mathrm{~d}, J=10.0 \mathrm{~Hz}, 1 \mathrm{H}), 4.42(\mathrm{dd}, J=7.6,1.7$ $\mathrm{Hz}, 1 \mathrm{H}), 4.22$ (q, $J=7.1 \mathrm{~Hz}, 2 \mathrm{H}), 3.04-3.12(\mathrm{~m}, 1 \mathrm{H}), 1.98(\mathrm{~d}, J=2.6 \mathrm{~Hz}, 1 \mathrm{H}), 1.91$ (d, $J=1.4 \mathrm{~Hz}, 3 \mathrm{H}), 1.31$ (t, $J=7.1 \mathrm{~Hz}, 3 \mathrm{H}), 0.87(\mathrm{~d}, J=6.7 \mathrm{~Hz}, 3 \mathrm{H}) .{ }^{13} \mathrm{C}$ NMR $(151 \mathrm{MHz}$, $\left.\mathrm{CDCl}_{3}\right) \delta 167.7,142.6,141.2$ (two overlapping carbon signals), 132.9, 128.7, 128.2, 127.2, 119.5, 79.1, 60.7, 40.8, 20.7, 17.9, 14.7. HRMS $\left(\mathrm{ESI}^{+}\right): \mathrm{m} / z$ for $\mathrm{C}_{17} \mathrm{H}_{22} \mathrm{O}_{3} \mathrm{Na}$ $[\mathrm{M}+\mathrm{Na}]^{+}$calcd. 297.1467, found 297.1473. 


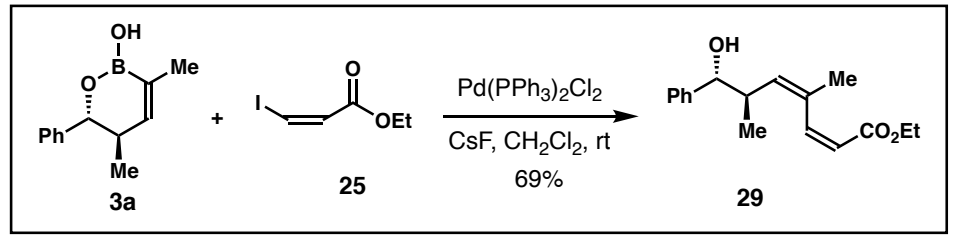

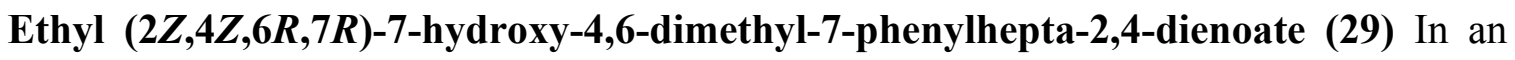
Ar-filled glove box, $\mathrm{Pd}\left(\mathrm{PPh}_{3}\right)_{2} \mathrm{Cl}_{2}(4 \mathrm{mg}, 5.0 \mu \mathrm{mol}, 5 \mathrm{~mol} \%)$ and $\mathrm{CsF}$ (30 mg, $0.2 \mathrm{mmol}$, 2.0 equiv) and a Teflon-coated magnetic stirring bar were sequentially added into a 1-dram vial. The vial was sealed with rubber septum and removed from the glove box. Then vinyl iodide 25 ( $23 \mathrm{mg}, 0.1 \mathrm{mmol}, 1.0$ equiv), and vinyl boron 3a (20 mg, $0.1 \mathrm{mmol}$, 1.0 equiv) in $\mathrm{CH}_{2} \mathrm{Cl}_{2}(0.5 \mathrm{~mL})$ was added to the vial under argon. The mixture was stirred for $12 \mathrm{~h}$ at ambient temperature. After completion of the reaction, the mixture was filtered through a pad of silica gel. The filtrate was concentrated under reduced pressure and the crude product was purified by preparative thin-layer chromatography (hexane/ethyl acetate $=10: 1$ ) to give product 29 in $69 \%$ yield $\left(19 \mathrm{mg}\right.$ ) as colorless oil. ${ }^{1} \mathrm{H}$ NMR (600 MHz, $\left.\mathrm{CDCl}_{3}\right) \delta 7.28-7.33(\mathrm{~m}, 4 \mathrm{H}), 7.24-7.26(\mathrm{~m}, 1 \mathrm{H}), 6.70(\mathrm{~d}, J=12.0 \mathrm{~Hz}$, $1 \mathrm{H}), 5.89$ (d, $J=12.0 \mathrm{~Hz}, 1 \mathrm{H}), 5.29$ (d, $J=10.6 \mathrm{~Hz}, 1 \mathrm{H}), 4.24$ (d, $J=8.7 \mathrm{~Hz}, 1 \mathrm{H}), 4.16-$ $4.22(\mathrm{~m}, 2 \mathrm{H}), 2.94(\mathrm{~s}, 1 \mathrm{H}), 2.50-2.56(\mathrm{~m}, 1 \mathrm{H}), 1.93(\mathrm{~s}, 3 \mathrm{H}), 1.29(\mathrm{t}, J=7.1 \mathrm{~Hz}, 3 \mathrm{H})$, $0.73(\mathrm{~d}, J=6.7 \mathrm{~Hz}, 3 \mathrm{H}) .{ }^{13} \mathrm{C} \operatorname{NMR}\left(151 \mathrm{MHz}, \mathrm{CDCl}_{3}\right) \delta 166.6,144.9,143.0,135.4$, $131.4,128.5,127.9,127.5,121.5,79.2,60.9,43.2,23.1,17.3,14.5$. HRMS $\left(\mathrm{ESI}^{+}\right): \mathrm{m} / z$ for $\mathrm{C}_{17} \mathrm{H}_{22} \mathrm{O}_{3} \mathrm{Na}[\mathrm{M}+\mathrm{Na}]^{+}$calcd. 297.1467, found 297.1477.

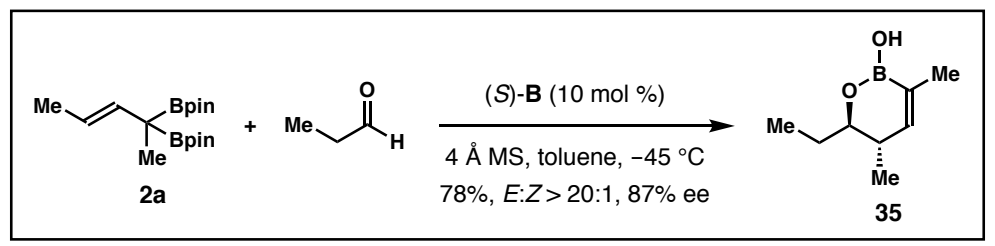

(5R,6S)-6-Ethyl-3,5-dimethyl-5,6-dihydro-2H-1,2-oxaborinin-2-ol (35) Prepared according to the general procedure for synthesis of $\mathbf{3}$ with $10 \mathrm{~mol} \%(S)$-B as the catalyst. The crude mixture was purified by column chromatography (pentane/ $\mathrm{Et}_{2} \mathrm{O}=30: 1$ to 10:1) to give the title compound in $78 \%$ yield $(12 \mathrm{mg}, E / Z>20: 1)$ as colorless oil. Enantiomeric excess of $\mathbf{3 5}$ was determined by HPLC analysis of the corresponding phenylation product to be $87 \%$ ee $\left(254 \mathrm{~nm}, 25^{\circ} \mathrm{C}\right) ; \mathrm{t}_{1}=4.87 \mathrm{~min}, \mathrm{t}_{2}=5.13 \mathrm{~min}$ [(Chiralpak IC) hexane/i-PrOH, 98:2, $1.0 \mathrm{~mL} / \mathrm{min}] ;[\alpha]_{\mathrm{D}}{ }^{20}=+30.0^{\circ}\left(\mathrm{c} 0.50, \mathrm{CHCl}_{3}\right) ;{ }^{1} \mathrm{H}$ NMR (600 MHz, $\left.\mathrm{CDCl}_{3}\right) \delta 6.24(\mathrm{~s}, 1 \mathrm{H}), 4.07$ (s, 1H), 3.59 (ddd, $\left.J=4.0,4.0,3.7 \mathrm{~Hz}, 1 \mathrm{H}\right)$, $2.24-2.29(\mathrm{~m}, 1 \mathrm{H}), 1.75(\mathrm{~s}, 3 \mathrm{H}), 1.61-1.67(\mathrm{~m}, 1 \mathrm{H}), 1.48-1.55(\mathrm{~m}, 1 \mathrm{H}), 1.00(\mathrm{~d}, J=$ 
$7.2 \mathrm{~Hz}, 3 \mathrm{H}), 0.96(\mathrm{t}, J=7.4 \mathrm{~Hz}, 3 \mathrm{H}) .{ }^{13} \mathrm{C} \mathrm{NMR}\left(151 \mathrm{MHz}, \mathrm{CDCl}_{3}\right) \delta 150.3,81.3,37.1$, 28.0, 18.9, 18.7, 9.9. HRMS (ESI $\left.{ }^{+}\right): m / z$ for $\mathrm{C}_{8} \mathrm{H}_{15} \mathrm{BO}_{2} \mathrm{Na}[\mathrm{M}+\mathrm{Na}]^{+}$calcd. 177.1063, found 177.0916.

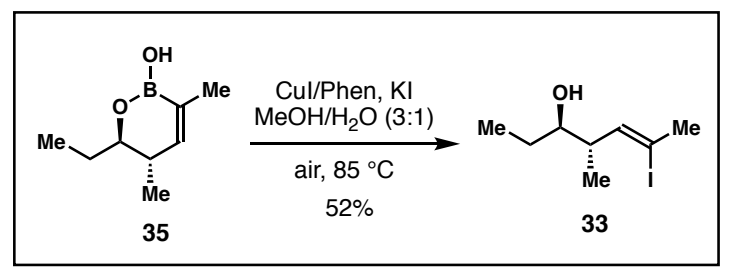

(3R,4S,Z)-6-Iodo-4-methylhept-5-en-3-ol (33) CuI (10 mg, $0.05 \mathrm{mmol}, 0.1$ equiv), 1,10-phenanthroline (18 mg, $0.1 \mathrm{mmol}, 0.2$ equiv) and a Teflon-coated magnetic stirring bar were added to a reaction flask. Compound $\mathbf{3 5}$ was dissolved in methanol (3 $\mathrm{mL})$ and transferred to the flask. KI (125 mg, $0.75 \mathrm{mmol}, 1.5$ equiv) was dissolved in water (1.0 $\mathrm{mL}$ ) and the solution was added to the reaction flask. The flask was sealed with a rubber septum and the resulting mixture was heated at $85^{\circ} \mathrm{C}$ for $12 \mathrm{~h}$. After completion of the reaction, the mixture was cooled to ambient temperature. Water $(25 \mathrm{~mL})$ was added and the mixture was extracted with $\mathrm{Et}_{2} \mathrm{O}(3 \times 20 \mathrm{~mL})$. The combined organic phases were washed with brine $(25 \mathrm{~mL})$, dried with anhydrous $\mathrm{Na}_{2} \mathrm{SO}_{4}$, and concentrated under reduced pressure. The crude product was purified by flash column chromatography (gradient elution with hexane/ethyl acetate, 50:1 to 10:1) to afford $Z$-vinyl iodide $\mathbf{3 3}$ as a colorless oil in $52 \%$ yield $(66 \mathrm{mg})$. The $E$-vinyl iodide side product was also isolated in $19 \%$ yield. ${ }^{1} \mathrm{H}$ NMR $\left(500 \mathrm{MHz}, \mathrm{CDCl}_{3}\right) \delta 5.37(\mathrm{~d}, J=9.0 \mathrm{~Hz}, 1 \mathrm{H}), 3.44-3.48(\mathrm{~m}, 1 \mathrm{H})$, $2.52(\mathrm{~s}, 3 \mathrm{H}), 2.41-2.49(\mathrm{~m}, 1 \mathrm{H}), 1.49-1.56(\mathrm{~m}, 1 \mathrm{H}), 1.37-1.46(\mathrm{~m}, 2 \mathrm{H}), 1.02(\mathrm{~d}, J=$ $6.8 \mathrm{~Hz}, 3 \mathrm{H}), 0.97(\mathrm{t}, J=7.3 \mathrm{~Hz}, 3 \mathrm{H}) .{ }^{13} \mathrm{C} \mathrm{NMR}\left(126 \mathrm{MHz}, \mathrm{CDCl}_{3}\right) \delta 137.1,102.0,77.0$, 47.2, 34.2, 28.0, 16.7, 10.5. HRMS $\left(\mathrm{EI}^{+}\right): m / z$ for $\mathrm{C}_{8} \mathrm{H}_{15} \mathrm{OI}[\mathrm{M}]^{+}$calcd. 254.0168, found 254.0173 .

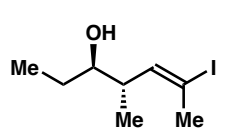

$(3 \boldsymbol{R}, 4 \boldsymbol{S}, \boldsymbol{E})$-6-Iodo-4-methylhept-5-en-3-ol ${ }^{1} \mathrm{H}$ NMR $\left(600 \mathrm{MHz}, \mathrm{CDCl}_{3}\right) \delta$ $6.10(\mathrm{dq}, J=10.1,1.4 \mathrm{~Hz}, 1 \mathrm{H}), 3.31-3.34(\mathrm{~m}, 1 \mathrm{H}), 2.44-2.50(\mathrm{~m}, 1 \mathrm{H})$, $2.41(\mathrm{~d}, J=1.5 \mathrm{~Hz}, 3 \mathrm{H}), 1.51-1.58(\mathrm{~m}, 1 \mathrm{H}), 1.50$ (brs, $1 \mathrm{H}), 1.36-1.44$ $(\mathrm{m}, 1 \mathrm{H}), 1.00(\mathrm{~d}, J=6.8 \mathrm{~Hz}, 3 \mathrm{H}), 0.96(\mathrm{t}, J=7.4 \mathrm{~Hz}, 3 \mathrm{H}) .{ }^{13} \mathrm{C} \mathrm{NMR}\left(126 \mathrm{MHz}, \mathrm{CDCl}_{3}\right)$ $\delta 143.3,95.3,76.7,41.6,28.4,27.6,17.0,10.4$. HRMS $\left(\mathrm{EI}^{+}\right): m / z$ for $\mathrm{C}_{8} \mathrm{H}_{15} \mathrm{OI}[\mathrm{M}]^{+}$calcd. 254.0168 , found 254.0173 . 


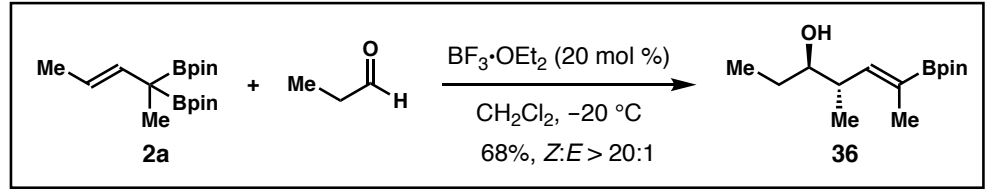

(3R,4S,Z)-4-Methyl-6-(4,4,5,5-tetramethyl-1,3,2-dioxaborolan-2-yl)hept-5-en-3-ol (36) Prepared according to the general procedure for synthesis of 4 ( $0.5 \mathrm{mmol}$ scale $)$. The crude mixture was purified by column chromatography to give the title compound in $68 \%$ yield (86 mg, $Z / E>20: 1)$ as colorless oil. ${ }^{1} \mathrm{H}$ NMR $\left(600 \mathrm{MHz}, \mathrm{CDCl}_{3}\right) \delta 6.16(\mathrm{~d}, J=9.9$ $\mathrm{Hz}, 1 \mathrm{H}), 3.32-3.36(\mathrm{~m}, 1 \mathrm{H}), 2.62-2.68(\mathrm{~m}, 1 \mathrm{H}), 1.72(\mathrm{~s}, 3 \mathrm{H}), 1.57-1.62(\mathrm{~m}, 1 \mathrm{H}), 1.57$ $(\mathrm{d}, J=3.2 \mathrm{~Hz}, 1 \mathrm{H}), 1.35-1.43(\mathrm{~m}, 1 \mathrm{H}), 1.26(\mathrm{~s}, 12 \mathrm{H}), 0.95-0.97(\mathrm{~m}, 6 \mathrm{H}) .{ }^{13} \mathrm{C} \mathrm{NMR}$ $\left(151 \mathrm{MHz}, \mathrm{CDCl}_{3}\right) \delta 147.8,83.6,76.9,39.0,27.1,25.2,25.1,16.7,14.8,10.3$. HRMS $\left(\mathrm{ESI}^{+}\right): m / z$ for $\mathrm{C}_{14} \mathrm{H}_{27} \mathrm{BO}_{3} \mathrm{Na}[\mathrm{M}+\mathrm{Na}]^{+}$calcd. 277.1951, found 277.1953.

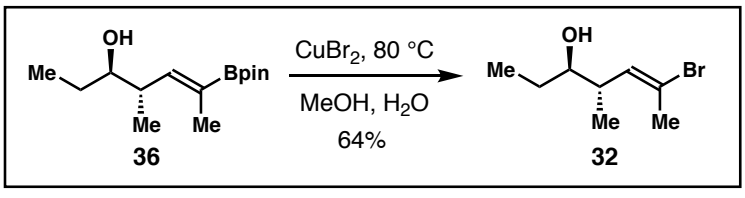

(3R,4S,E)-6-bromo-4-methylhept-5-en-3-ol (32) To a $25 \mathrm{~mL}$ reaction flask containing a Teflon-coated magnetic stirring bar were added $E$-vinylboronate 36 (127 mg, $0.5 \mathrm{mmol}$, 1.0 equiv), $\mathrm{CuBr}_{2}$ (442 mg, $2.0 \mathrm{mmol}, 4.0$ equiv), $\mathrm{MeOH}(4 \mathrm{~mL})$ and $\mathrm{H}_{2} \mathrm{O}(4 \mathrm{~mL})$. The reaction was stirred at $80{ }^{\circ} \mathrm{C}$ for $18 \mathrm{~h}$. After cooling to ambient temperature, $\mathrm{H}_{2} \mathrm{O}(20 \mathrm{~mL})$ was added to the flask. The reaction mixture was extracted with EtOAc $(3 \times 15 \mathrm{~mL})$. The combined extracts were concentrated under reduced pressure. The crude product was purified by column chromatography (gradient elution with pentane/ether, 30:1 to 10:1) to give 32 in $64 \%$ yield (66 mg) as colorless oil. A small amount of $Z$-vinyl bromide (15\%) was also isolated. ${ }^{1} \mathrm{H} \mathrm{NMR}\left(600 \mathrm{MHz}, \mathrm{CDCl}_{3}\right) \delta 5.77-5.79(\mathrm{~m}, 1 \mathrm{H}), 3.32-3.36(\mathrm{~m}$, $1 \mathrm{H}), 2.38-2.44(\mathrm{~m}, 1 \mathrm{H}), 2.25(\mathrm{~d}, J=1.2 \mathrm{~Hz}, 3 \mathrm{H}), 1.52-1.58(\mathrm{~m}, 1 \mathrm{H}), 1.50(\mathrm{~d}, J=4.1$ $\mathrm{Hz}, 1 \mathrm{H}), 1.37-1.44(\mathrm{~m}, 1 \mathrm{H}), 1.01(\mathrm{~d}, J=6.9 \mathrm{~Hz}, 3 \mathrm{H}), 0.96(\mathrm{t}, J=7.4 \mathrm{~Hz}, 3 \mathrm{H}) .{ }^{13} \mathrm{C} \mathrm{NMR}$ $\left(101 \mathrm{MHz} \mathrm{CDCl}_{3}\right) \delta 134.3,120.9,76.8,40.8,27.6,24.1,17.2,10.4$. HRMS $\left(\mathrm{EI}^{+}\right): \mathrm{m} / z$ for $\mathrm{C}_{8} \mathrm{H}_{15} \mathrm{OBr}[\mathrm{M}]^{+}$calcd. 206.0306, found 206.0310.

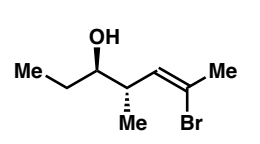

(3R,4S,Z)-6-Bromo-4-methylhept-5-en-3-ol ${ }^{1} \mathrm{H}$ NMR $\left(500 \mathrm{MHz}, \mathrm{CDCl}_{3}\right)$ $\delta 5.58(\mathrm{~d}, J=9.2 \mathrm{~Hz}, 1 \mathrm{H}), 3.41-3.45(\mathrm{~m}, 1 \mathrm{H}), 2.63-2.70(\mathrm{~m}, 1 \mathrm{H}), 2.30$ $(\mathrm{s}, 3 \mathrm{H}), 1.37-1.58(\mathrm{~m}, 3 \mathrm{H}), 1.03(\mathrm{~d}, J=6.9 \mathrm{~Hz}, 3 \mathrm{H}), 0.97(\mathrm{t}, J=7.4 \mathrm{~Hz}$, 3H). ${ }^{13} \mathrm{C}$ NMR $\left(126 \mathrm{MHz}, \mathrm{CDCl}_{3}\right) \delta 130.7,123.5,77.1,42.3,29.4,28.1,16.8,10.4$. HRMS (EI $\left.{ }^{+}\right): m / z$ for $\mathrm{C}_{8} \mathrm{H}_{15} \mathrm{OBr}[\mathrm{M}]^{+}$calcd. 206.0306, found 206.0311. 


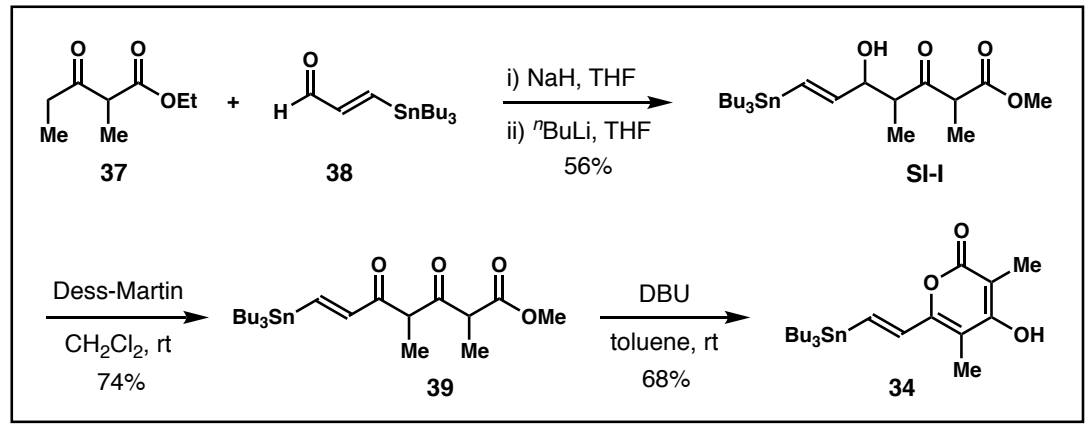

(E)-4-Hydroxy-3,5-dimethyl-6-(2-(tributylstannyl)vinyl)-2H-pyran-2-one (34) To a $25 \mathrm{~mL}$ reaction flask containing a Teflon-coated magnetic stirring bar were added $\mathrm{NaH}$ (40 mg, 60\% disp. in mineral oil, $1.0 \mathrm{mmol}$ ) and THF (3 mL) under argon. The reaction mixture was placed in an ice bath and stirred for $10 \mathrm{~min}$. Then ketoester $37^{2}$ (144 $\mathrm{mg}, 1.0$ mmol) in THF ( $1 \mathrm{~mL})$ was added to the flask at $0{ }^{\circ} \mathrm{C}$ under argon, and the resulting mixture was stirred for $5 \mathrm{~min}$. Then the reaction flask was placed in an acetone-dry ice bath and cooled to $-78^{\circ} \mathrm{C}$. After stirring at $-78{ }^{\circ} \mathrm{C}$ for $10 \mathrm{~min},{ }^{n} \mathrm{BuLi}(2.5 \mathrm{M}, 0.4 \mathrm{~mL}, 1.0$ mmol) was added. After stirring at $-78{ }^{\circ} \mathrm{C}$ for $30 \mathrm{~min}$, the reaction flask was placed in an ice bath and stirred for 10 minutes. Then the reaction mixture was cooled to $-78{ }^{\circ} \mathrm{C}$, and a solution of aldehyde $\mathbf{3 8}^{3}$ (345 $\left.\mathrm{mg}, 1.0 \mathrm{mmol}\right)$ in THF $(2.5 \mathrm{~mL})$ was added. The resulting mixture was stirred for 2 hours at $-78^{\circ} \mathrm{C}$. After completion of the reaction, a solution of $\mathrm{HCl}(0.5 \mathrm{M}, 5 \mathrm{~mL})$ was added to the reaction flask. The resulting mixture was warmed to ambient temperature. EtOAc $(5 \mathrm{~mL})$ was added and the organic layers were separated. The organic layer was washed with additional $\mathrm{HCl}$ solution $(0.5 \mathrm{M}, 5 \mathrm{~mL})$. The combined aqueous layers were extracted with EtOAc $(2 \times 5 \mathrm{~mL})$. The combined organic layers were dried over anhydrous $\mathrm{Na}_{2} \mathrm{SO}_{4}$, filtered, and concentrated. Purification of the crude product was performed by flash column chromatography (hexanes/EtOAc $=5: 1$ ) to give compound SI-I in 56\% yield (287 mg, a mixture of diastereomers). SI-I was used for the next step without further purification.

To a solution of alcohol SI-I (287 $\mathrm{mg}, 0.59 \mathrm{mmol}, 1.0$ equiv) in $\mathrm{CH}_{2} \mathrm{Cl}_{2}(5 \mathrm{~mL})$ was added Dess-Martin reagent (373 $\mathrm{mg}, 0.88 \mathrm{mmol}, 1.5$ equiv) at $0{ }^{\circ} \mathrm{C}$. The reaction mixture was stirred at $0{ }^{\circ} \mathrm{C}$ for $2 \mathrm{~h}$. Then saturated aqueous solution of $\mathrm{NaHCO}_{3}(3 \mathrm{~mL})$ and $\mathrm{Na}_{2} \mathrm{~S}_{2} \mathrm{O}_{3}(3 \mathrm{~mL})$ were added. The resulting mixture was stirred for $5 \mathrm{~min}$. The organic layer was separated, and the aqueous layer was extracted with $\mathrm{Et}_{2} \mathrm{O}$ ( 3 x $5 \mathrm{~mL}$ ). The combined organic layers were dried over anhydrous $\mathrm{Na}_{2} \mathrm{SO}_{4}$, filtered, and concentrated. Purification of the crude product was performed by flash column chromatography to give ketoester 39 in 74\% yield (210 mg, a mixture of diastereomers) as colorless oil. Ketoester 39 was used for the next step without further purification. 
Ketoester 39 (98 mg, $0.2 \mathrm{mmol}, 1.0$ equiv) was dissolved in toluene (2 mL) in a reaction flask. DBU $(60 \mu \mathrm{L}, 0.4 \mathrm{mmol})$ was added and the mixture was stirred ambient temperature for $2 \mathrm{~h}$. After completion of the reaction, a solution of $\mathrm{HCl}(1 \mathrm{M}, 1 \mathrm{~mL})$ was added. The organic layer was separated and the aqueous layer was extracted with $\mathrm{Et}_{2} \mathrm{O}$ (3 $\times 5 \mathrm{~mL}$ ). The combined organic layers were dried over anhydrous $\mathrm{Na}_{2} \mathrm{SO}_{4}$, filtered, and concentrated. Purification of the crude product was performed by flash column chromatography (hexanes/EtOAc $=10: 1$ to $3: 1$ ) gave vinyl stannane 34 in 68\% yield (62 $\mathrm{mg})$ as yellow oil. ${ }^{1} \mathrm{H} \mathrm{NMR}\left(600 \mathrm{MHz}, \mathrm{CDCl}_{3}\right) \delta 7.39(\mathrm{~d}, J=19.1 \mathrm{~Hz}, 1 \mathrm{H}), 6.75(\mathrm{~d}, J=$ $19.1 \mathrm{~Hz}, 1 \mathrm{H}), 2.08$ (s, 3H), 2.03 (s, 3H), $1.49-1.54(\mathrm{~m}, 6 \mathrm{H}), 1.31$ (h, J=7.3 Hz, 6H), $0.92-1.02(\mathrm{~m}, 6 \mathrm{H}), 0.89(\mathrm{t}, J=7.3 \mathrm{~Hz}, 9 \mathrm{H}) .{ }^{13} \mathrm{C} \mathrm{NMR}\left(126 \mathrm{MHz}, \mathrm{CDCl}_{3}\right) \delta 165.3,164.7$, $151.9,141.1,133.2,106.7,100.2,29.4,27.6,14.0,10.0,9.5,9.0$. HRMS $\left(\mathrm{ESI}^{+}\right): \mathrm{m} / z$ for $\mathrm{C}_{21} \mathrm{H}_{36} \mathrm{O}_{3} \mathrm{SnNa}[\mathrm{M}+\mathrm{Na}]^{+}$calcd. 479.1584, found 479.1570 .

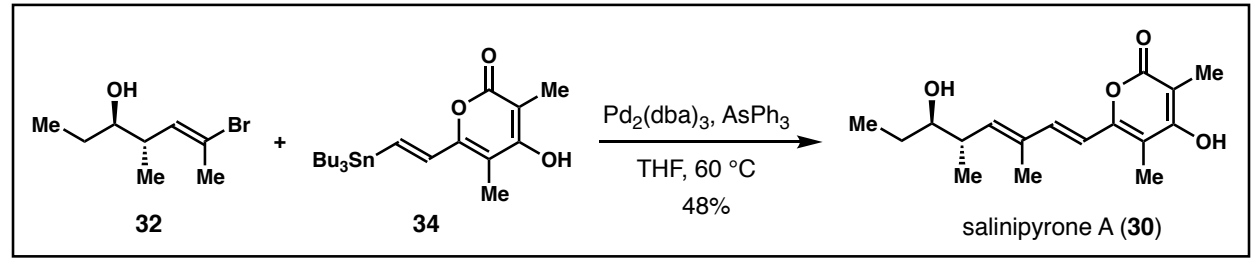

\section{4-Hydroxy-6-((1E,3E,5S,6R)-6-hydroxy-3,5-dimethylocta-1,3-dien-1-yl)-3,5-dimethyl} -2H-pyran-2-one (30) In an Ar-filled glove box, $\mathrm{Pd}_{2}(\mathrm{dba})_{3}(5 \mathrm{mg}, 0.005 \mathrm{mmol}, 0.1$ equiv), $\mathrm{Ph}_{3} \mathrm{As}$ (12 mg, $0.04 \mathrm{mmol}, 0.8$ equiv) and a Teflon-coated magnetic stirring bar were sequentially added into a reaction flask. The flask was sealed with a rubber septum and removed from glove box. Then a solution of vinyl bromide 32 (10 mg, $0.05 \mathrm{mmol}$, 1.0 equiv) and vinyl stannane 34 (34 mg, $0.075 \mathrm{mmol}, 1.5$ equiv) in THF ( $0.5 \mathrm{~mL})$ were added to the mixture under argon. The reaction mixture was stirred at $60{ }^{\circ} \mathrm{C}$ under argon for $6 \mathrm{~h}$. After complete consumption of vinyl bromide 32, the reaction mixture was filtered through a short pad of silica gel. The filtrate was concentrated under reduced pressure. Purification of the crude product was performed by flash chromatography (gradient elution with hexane and ethyl acetate, 2:1 to 1:2) to provide salinipyrone A (30) in $48 \%$ yield $(7 \mathrm{mg}) .{ }^{1} \mathrm{H}$ NMR $\left(600 \mathrm{MHz}\right.$, Methanol- $\left.d_{4}\right) \delta 7.13(\mathrm{~d}, J=15.6 \mathrm{~Hz}, 1 \mathrm{H}), 6.41$ $(\mathrm{d}, J=15.6 \mathrm{~Hz}, 1 \mathrm{H}), 5.83$ (d, $J=9.5 \mathrm{~Hz}, 1 \mathrm{H}), 3.42$ (dt, $J=8.4,4.2 \mathrm{~Hz}, 1 \mathrm{H}), 2.69-2.72$ $(\mathrm{m}, 1 \mathrm{H}), 2.08(\mathrm{~s}, 3 \mathrm{H}), 1.97(\mathrm{~s}, 3 \mathrm{H}), 1.91(\mathrm{~s}, 3 \mathrm{H}), 1.48-1.55(\mathrm{~m}, 1 \mathrm{H}), 1.38-1.45(\mathrm{~m}, 1 \mathrm{H})$, $1.07(\mathrm{~d}, J=6.9 \mathrm{~Hz}, 3 \mathrm{H}), 0.98(\mathrm{t}, J=7.4 \mathrm{~Hz}, 3 \mathrm{H}) .{ }^{13} \mathrm{C}$ NMR $\left(126 \mathrm{MHz}\right.$, Methanol- $\left.d_{4}\right) \delta$ 168.2 , 167.6, 154.3, 142.0, 140.7, 134.9, 115.1, 109.9, 99.9, 78.0, 40.0, 28.6, 17.5, 12.7, 10.8, 9.6, 9.1. HRMS (ESI $\left.{ }^{+}\right): m / z$ for $\mathrm{C}_{17} \mathrm{H}_{25} \mathrm{O}_{4}[\mathrm{M}+\mathrm{H}]^{+}$calcd. 293.1753, found 293.1756. 


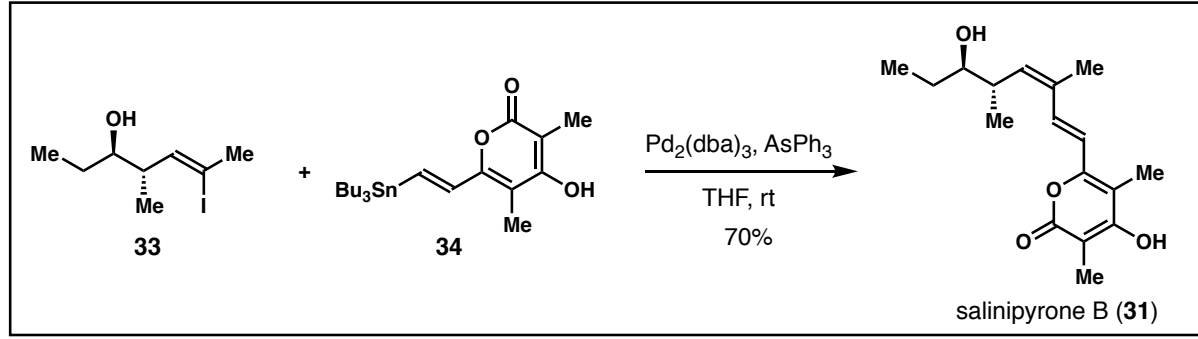

\section{4-Hydroxy-6-((1E,3Z,5S,6R)-6-hydroxy-3,5-dimethylocta-1,3-dien-1-yl)-3,5-dimethyl}

-2H-pyran-2-one (31) In an Ar-filled glove box, $\mathrm{Pd}_{2}(\mathrm{dba})_{3}(9 \mathrm{mg}, 0.01 \mathrm{mmol}, 0.2$ equiv), $\mathrm{Ph}_{3}$ As (24 mg, $0.08 \mathrm{mmol}, 1.6$ equiv) and a Teflon-coated magnetic stirring bar were sequentially added into a reaction flask. The flask was sealed with a rubber septum and removed from glove box. Then a solution of vinyl stannane 34 (46 mg, $0.1 \mathrm{mmol}, 2.0$ equiv) and vinyl iodide 33 (13 mg, $0.05 \mathrm{mmol}, 1.0$ equiv) in THF (0.5 mL) were added to the mixture under argon. The reaction mixture was stirred at ambient temperature for 24 h. After complete consumption of vinyl iodide $\mathbf{3 3}$, the reaction mixture was filtered through a short pad of silica gel. The filtrate was concentrated under reduced pressure. Purification of the crude product was performed by flash chromatography (gradient elution with hexane and ethyl acetate, $5: 1$ to $1: 2$ ) to give salinipyrone B (31) in $70 \%$ yield $(11 \mathrm{mg})$ as a yellow solid. The spectroscopic data of synthetic salinipyrone B were in excellent agreement with the data previously reported for the natural product. ${ }^{1} \mathrm{H}$ NMR (600 MHz, Methanol-d 4 ) $\delta 7.52(\mathrm{~d}, J=15.3 \mathrm{~Hz}, 1 \mathrm{H}), 6.49$ (d, $J=15.4 \mathrm{~Hz}, 1 \mathrm{H}), 5.64$ (d, $J$ $=10.2 \mathrm{~Hz}, 1 \mathrm{H}), 3.39(\mathrm{dt}, J=8.4,4.1 \mathrm{~Hz}, 1 \mathrm{H}), 2.89-2.94(\mathrm{~m}, 1 \mathrm{H}), 2.06(\mathrm{~s}, 3 \mathrm{H}), 1.95(\mathrm{~s}$, $6 \mathrm{H}), 1.45-1.51(\mathrm{~m}, 1 \mathrm{H}), 1.33-1.41(\mathrm{~m}, 1 \mathrm{H}), 1.06(\mathrm{~d}, J=6.8 \mathrm{~Hz}, 3 \mathrm{H}), 0.95(\mathrm{t}, J=7.4$ $\mathrm{Hz}, 3 \mathrm{H}) .{ }^{13} \mathrm{C}$ NMR $\left(126 \mathrm{MHz}\right.$, Methanol- $\left.d_{4}\right) \delta 168.0,167.7,154.3,139.9,133.1,132.6$, 117.6, 110.6, 100.5, 78.3, 39.0, 28.8, 20.6, 18.3, 11.1, 9.8, 9.3. HRMS $\left(\mathrm{ESI}^{+}\right): \mathrm{m} / \mathrm{z}$ for $\mathrm{C}_{17} \mathrm{H}_{24} \mathrm{O}_{4} \mathrm{Na}[\mathrm{M}+\mathrm{Na}]^{+}$calcd. 315.1572, found 315.1579.

\section{Assignment of the absolute configuration of the secondary alcohol using modified}

\section{Mosher ester analysis: ${ }^{4}$}
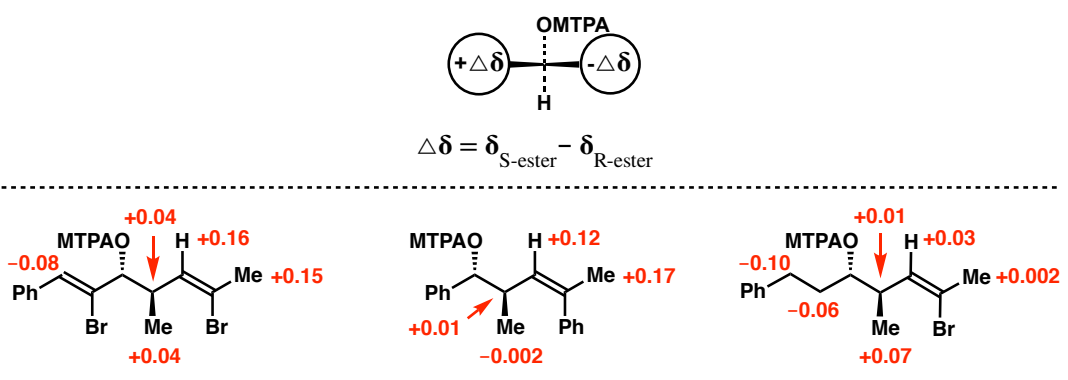


\section{Assignment of the relative configuration of compounds 3, 4a and 5a}

The relative configuration of compounds 3 was determined by coupling constant analysis. $J^{\mathrm{a}, \mathrm{b}}$ of compounds 3 (10.1-10.7 Hz) corresponds to the anti relative configuration.
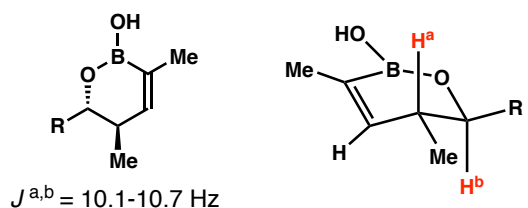

The anti relative configuration of compounds $\mathbf{4 a}$ was determined by the protodeboration products. Compound $\mathbf{4 a}$ was treated with HOAc to form protodeboration products SI-1 and SI-2. Both SI-1 and SI-2 are known compounds. The spectroscopic data of SI-1 and SI-2 match the reported literature data. (SI-1: J. Am. Chem. Soc. 2010, 132, 4025; SI-2: J. Am. Chem. Soc. 2010, 132, 4025, Angew. Chem. Int. Ed. 2008, 47, 8909).

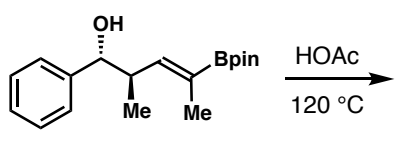

$4 a$

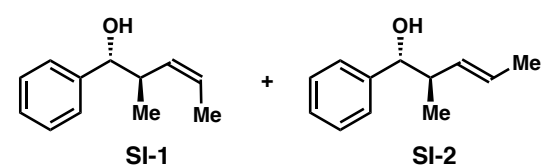

SI-1

SI-2

The syn-relative configuration of compounds 5a was determined by nOe studies of the ketal derivatives SI-3 and SI-4.

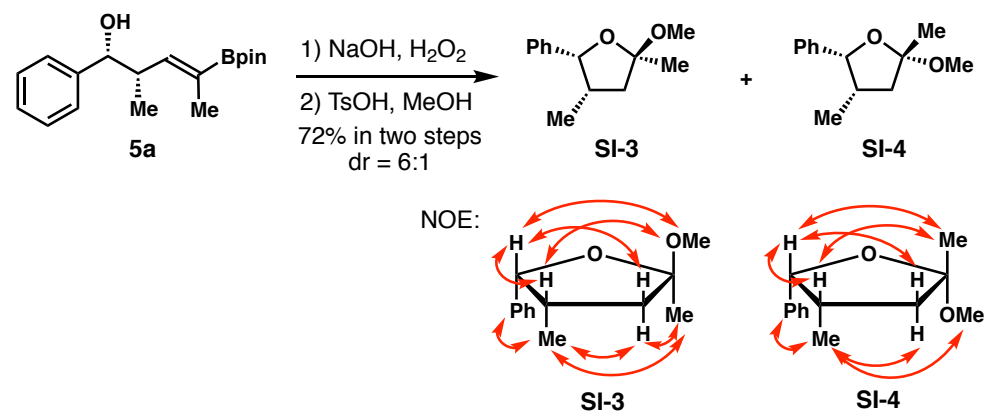




\section{Binding Mode and Transition State Analyses for Lewis Acid-Catalyzed Allylation with Reagent 2a}

As shown in Scheme SI-I, there are four non-bonded pairs of electrons on the oxygen atoms in the dioxaborolane unit of reagent-substrate complex (2a and aldehyde). Among these long pairs of electrons, coordination of a Lewis acid to the two lone pairs that project toward the top of reagent-aldehyde six-membered chelate is likely to be disfavored. In this scenario, steric interactions between the Lewis acid catalyst and two hydrogen atoms of the six-membered chelate (shown with a red arrow in Scheme SI-I-a, I and II) are developed regardless of the orientation of $\alpha$-Bpin group (either pseudo-equatorial in I or axial position in II).

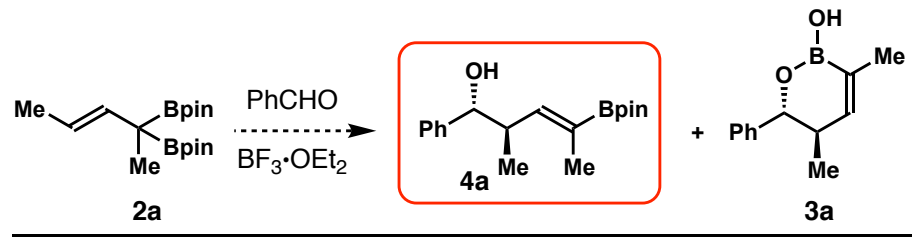

a) Lewis acid binding mode:
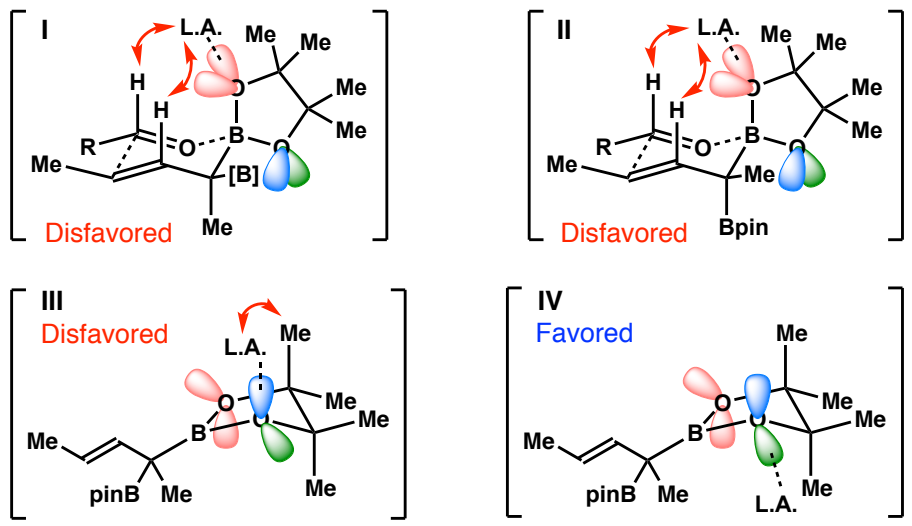

b) Transition state analysis:
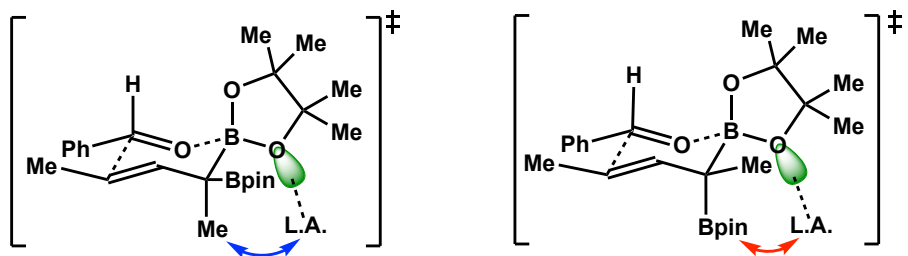

TS-8 (favored)
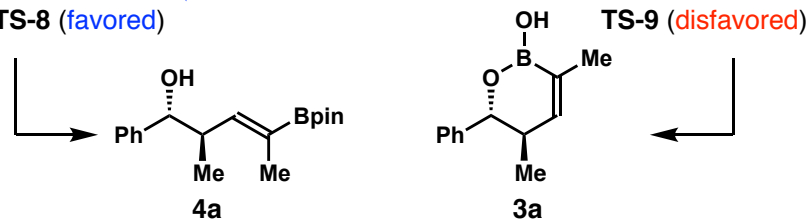

Scheme SI-I: Binding Mode and Transition State Analyses for Lewis Acid-Catalyzed Crotylation with Reagent 2a 
On the other hand, the five-membered dioxaborolane unit (Bpin) should adopt an envelope conformation to minimize the eclipse interactions of methyl groups as shown in Scheme SI-I-a III and IV. Lewis acid coordination to the lone pair of electrons of the oxygen atom that occupies a pseudo-axial position (shown in blue in Scheme SI-I-a, III) should be unfavorable because of apparent 1,3-diaxial steric interactions between the Lewis acid and the methyl group (shown with a red arrow in Scheme SI-I-a, III). Such interactions are not present when coordinating to the last lone pair (shown in green in Scheme SI-I-a, IV). Therefore, the lone pair of electrons shown in green is the most accessible for coordination to a Lewis acid.

As shown in Scheme SI-I-b, when a Lewis acid coordinates to the lone pair of electrons shown in green, there are two competing transition states TS-8 and TS-9 that lead to the formation of products $\mathbf{4 a}$ and 3a, respectively. In TS-8, 1,3-syn-pentane interactions (shown with a blue arrow in TS-8, Scheme SI-I-b) are developed between the pseudo-axially positioned methyl group and the Lewis acid catalyst. In the competing transition state TS-9, similar 1,3-syn-pentane interactions also exist between the pseudo-axially oriented $\alpha$-Bpin group and the Lewis acid (shown with a red arrow in TS-9, Scheme SI-I-b). It is apparent that steric interactions in the latter case are much more severe. However, gauche interactions between the pinacol moiety on boron and the pseudo-equatorially oriented substituent (the Bpin group in TS-8, or the methyl group in TS-9) should also be taken into account. Although the overall balance of syn-pentane and gauche interactions will ultimately determine the $E / Z$-selectivity of the reaction, we postulate that the syn-pentane interactions should be the dominant factor and TS-8 should be more favourable.

\section{Computational Studies on $\mathrm{BF}_{3}$-Catalyzed Crotylation with Reagent 2a}

To verify the validity of the binding mode and transition state analyses, DFT calculations at the M06-2X/6-311+G(d,p)-SMD(dichloromethane)//B3LYP-D3/6-31G(d) level of theory using Gaussian 09 with the ultrafine grid were conducted. As shown in Figure SI-I, in TS-8a and TS-8b, the Bpin group of reagent 2a is oriented in a pseudo equatorial position, leading to the formation of $Z$-isomer $4 \mathbf{a}$. And the Lewis acid $\mathrm{BF}_{3}$ coordinates to the oxygen atom in the pseudo axial position in TS-8a (the red lone pair in I and II of Scheme SI-I), where $\mathrm{BF}_{3}$ binds to the oxygen atom in the pseudo equatorial position in TS-8b (the green lone pair in IV of Scheme SI-I). In TS-9a and TS-9b, the Bpin group of $\mathbf{2 a}$ is oriented in a pseudo axial position, leading to the formation of $E$-product 3a. Lewis 
acid $\mathrm{BF}_{3}$ binds to the pseudo axial oxygen atom in TS-9a, and to the pseudo equatorial oxygen atom in TS-9b. Intriguingly, the calculations predict that the favored binding mode of $\mathrm{BF}_{3}$ is coordination to the pseudo axial oxygen atom in TS-8a and TS-9a. The energy of TS-8b with coordination to the pseudo equatorial oxygen atom is substantially higher than the energy of TS-8a. And TS-9b is also higher in energy than TS-9a. Such a preference is different from the analyses shown in Scheme SI-I. As shown in Figure SI-I, we attribute the discrepancy to the additional electrostatic attractions in TS-8a and TS-9a, where $\mathrm{BF}_{3}$ has favorable interactions with the hydrogen atom of the aldehyde. Such electrostatic interactions lower the energies of transition states TS-8a and TS-9a (compared to TS-8b and TS-9b). Among the four computed the transition states, TS-9a with the Bpin group occupying a pseudo axial position has the lowest energy. Because TS-9a lacks 1,3-syn-pentane interactions between the Bpin group and $\mathrm{BF}_{3}$ as is in TS-9 of Scheme SI-I, the sterically disfavored gauche interaction dictates the pseudo axial orientation of the Bpin group in TS-9a. Therefore the formation of $E$-isomer 3a should be favored based on the calculations.

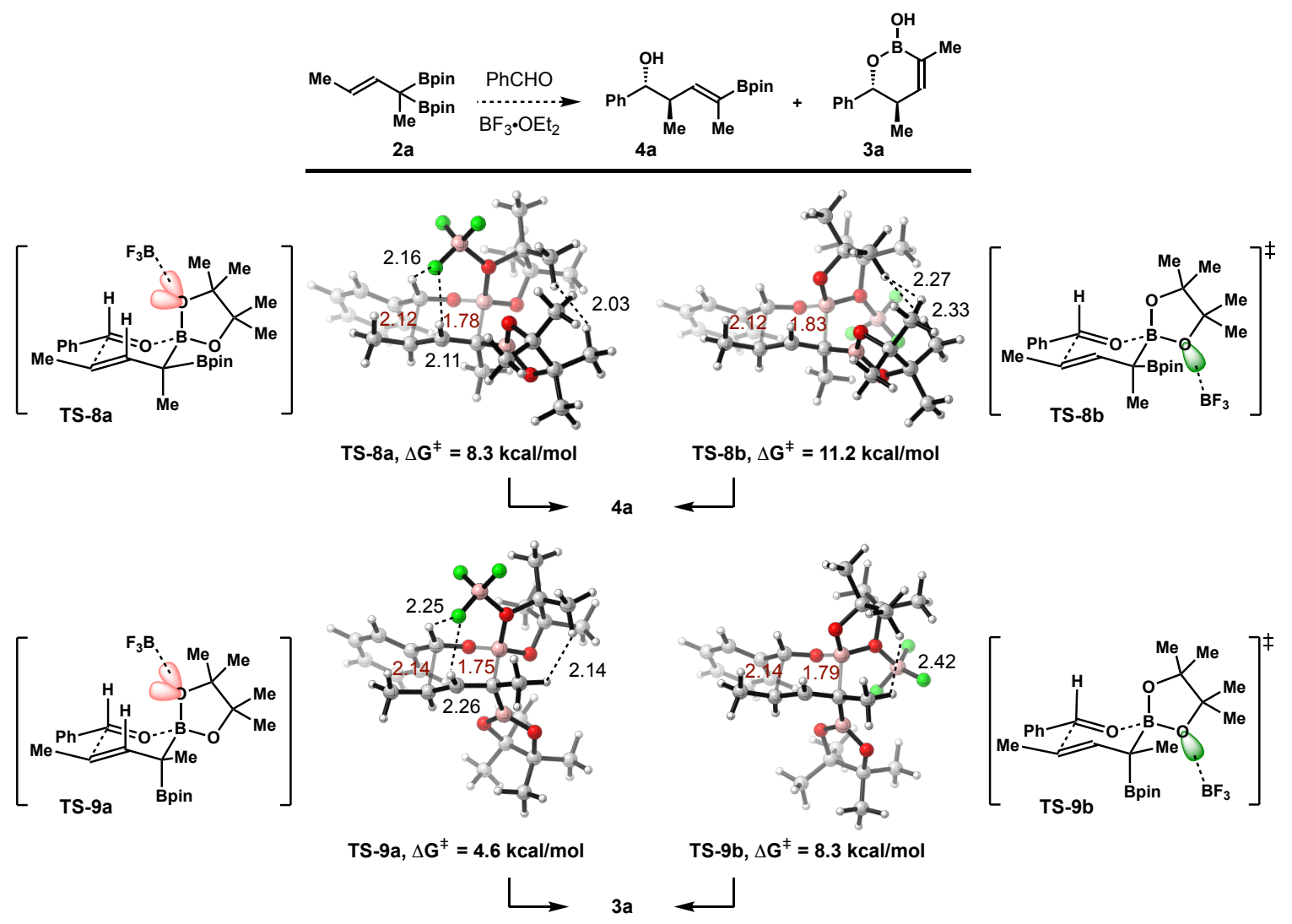

Figure SI-I: Optimized transition states TS-8a, TS-8b, TS-9a, and TS-9b of $\mathrm{BF}_{3}$-catalyzed crotylation of benzaldehyde with crotylboronate $\mathbf{2 a}$. The bond lengths are in ångstrom. 
We next considered the option of $\mathrm{BF}_{3}$ coordinating to the oxygen atom of benzaldehyde carbonyl group. And computational results are shown in Figure SI-II. In this case, the computed energy is $17.4 \mathrm{kcal} / \mathrm{mol}$ for transition state TS-8c and $23.5 \mathrm{kcal} / \mathrm{mol}$ for TS-9c. The formation of $4 \mathbf{a}$ should therefore be favored. However, the energies of both transition states (TS-8c and TS-9c) are significantly higher the transition states energies in Scheme SI-I presumably because of the lack of electrostatic interactions between $\mathrm{BF}_{3}$ and hydrogen atom of benzaldehyde in TS-8c and TS-9c. Even though the results from this binding mode are consistent with the experimental data (4a is the favored product), the high energies of the transition states of TS-8c and TS-9c suggest the reaction may not proceed through this pathway.

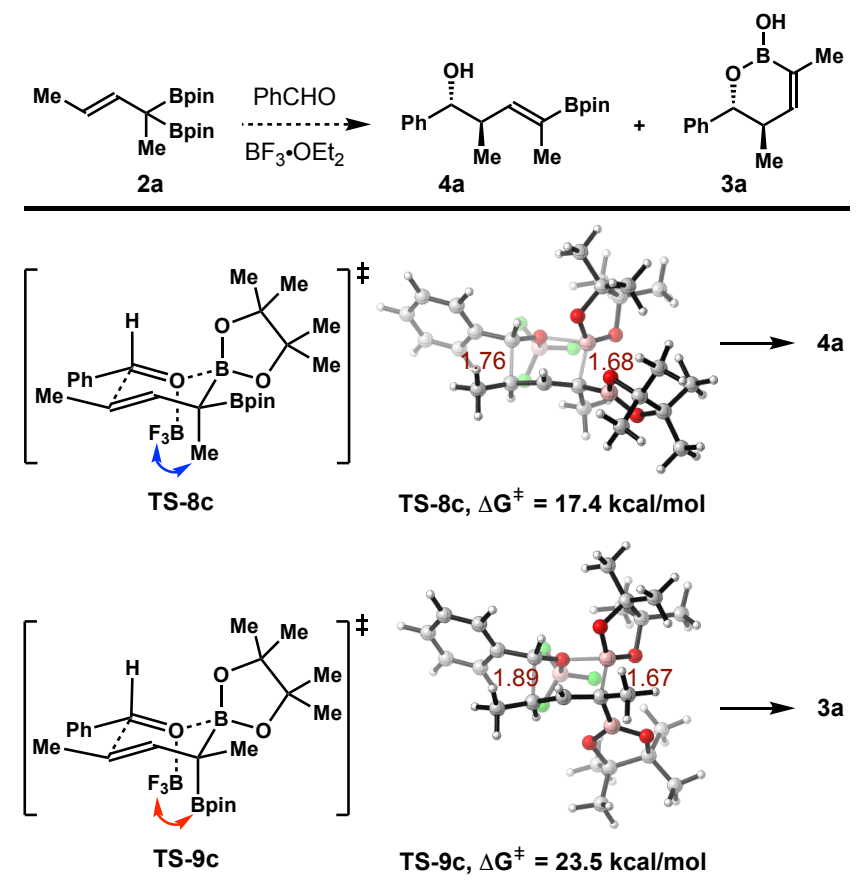

Figure SI-II: Optimized transition states TS-8c, and TS-9c of $\mathrm{BF}_{3}$-catalyzed crotylation of benzaldehyde with crotylboronate $\mathbf{2 a}$ with $\mathrm{BF}_{3}$ coordinating to the carbonyl group. The bond lengths are in ångstrom. 


\section{Computational Studies on $\mathbf{B F}_{3}-$ Catalyzed Crotylation with Reagent $\mathbf{2 b}$}

To probe the origin of $\mathrm{BF}_{3}$-catalyzed $E$-selective crotylation with reagent $\mathbf{2 b}$, DFT calculations at the M06-2X/6-311+G(d,p)-SMD(dichloromethane)//B3LYP-D3/6-31G(d) level of theory using Gaussian 09 with the ultrafine grid were also conducted. The results are shown in Figure SI-III. Again, the calculations predict the formation of $E$-isomer $\mathbf{6 a}$ is favored, although the experimental results are $Z$-selective, favoring the formation of 5a. Electrostatic attractions in TS-10a and TS-11a may result in this discrepancy. At current stage, the stereochemical model shown in Scheme 7 in the manuscript seems to be consistent with the experimental data. However, a more complex scenario may be involved in $\mathrm{BF}_{3}$-catalyzed crotylation with either reagent $\mathbf{2 a}$ or $\mathbf{2 b}$.

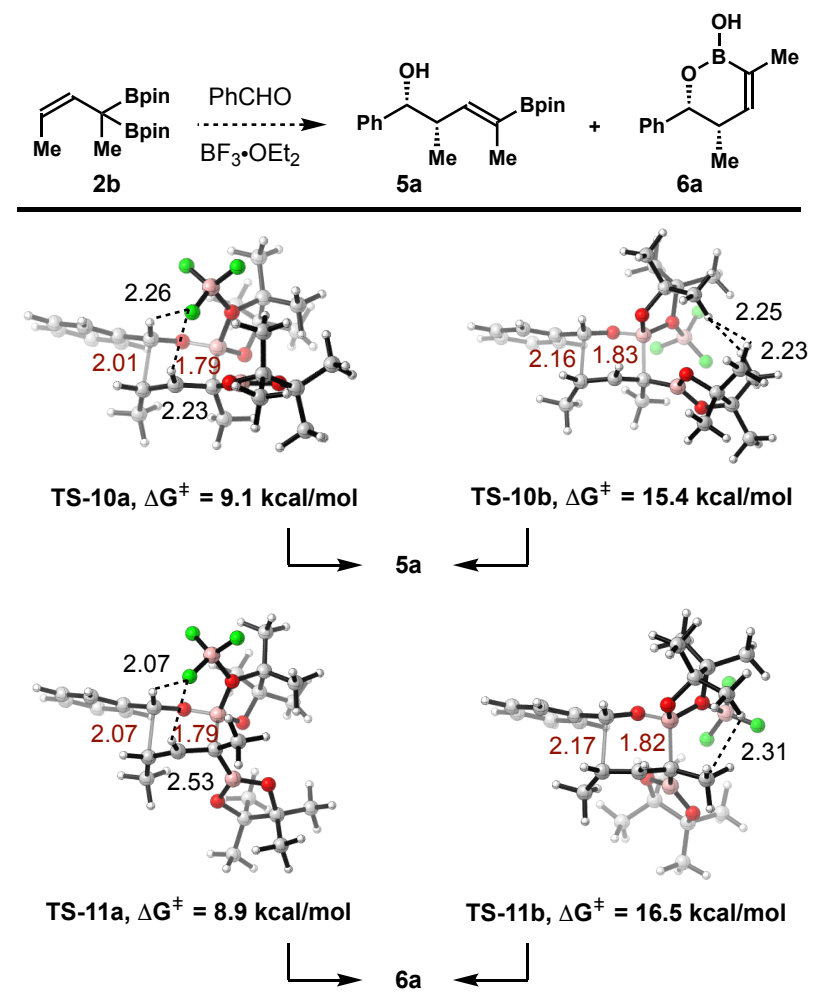

Figure SI-III. Optimized transition states TS-10a, TS-10b, TS-11a, and TS-11b of $\mathrm{BF}_{3}$-catalyzed crotylation of benzaldehyde with crotylboronate $\mathbf{2 b}$. The bond lengths are in Ångstrom. 


\section{Computational Studies:}

\section{Absolute Calculation Energies, Enthalpies, and Free Energies for Chiral Phosphoric Acid-Catalyzed Allylation}

\begin{tabular}{lccccc}
\hline Geometry & $\mathrm{E}_{(\text {elec-B3LYP-D3) }}{ }^{1}$ & $\mathrm{H}_{(\text {(corr-B3LYP-D3) }}{ }^{2}$ & $\mathrm{G}_{\text {(corr-B3LYP-D3) }}{ }^{3}$ & $\mathrm{E}_{(\text {solv-M062X) }}{ }^{4}$ & $\mathrm{IF}^{5}$ \\
\hline $\mathbf{R}$-A & -2582.2220 & 1.0121 & 0.8680 & -2581.7405 & - \\
$\mathbf{2 a}$ & -1017.9716 & 0.5111 & 0.4289 & -1017.7643 & - \\
PhCHO & -345.5815 & 0.1174 & 0.0797 & -345.5262 & - \\
TS-3 & -1363.5629 & 0.6301 & 0.5383 & -1363.2883 & -259.00 \\
TS-4 & -1363.5578 & 0.6302 & 0.5377 & -1363.2864 & -288.71 \\
TS-5 & -3945.8554 & 1.6450 & 1.4367 & -3945.0722 & -285.34 \\
TS-6 & -3945.8552 & 1.6456 & 1.4401 & -3945.0725 & -317.01 \\
TS-7 & -3945.8407 & 1.6456 & 1.4370 & -3945.0610 & -215.84 \\
\hline
\end{tabular}

${ }^{1}$ The electronic energy calculated by B3LYP-D3 in gas phase. ${ }^{2}$ The thermal correction to enthalpy calculated by B3LYP-D3 in gas phase. ${ }^{3}$ The thermal correction to Gibbs free energy calculated by B3LYP-D3 in gas phase. ${ }^{4}$ The electronic energy calculated by M062X in toluene solvent. ${ }^{5}$ The B3LYP-D3 calculated imaginary frequencies for the transition states.

2. Absolute Calculation Energies, Enthalpies, and Free Energies for $\mathrm{BF}_{3}$-Catalyzed Allylation

\begin{tabular}{crrrrr}
\hline Geometry & $\mathrm{E}_{(\text {elec-B3LYP-D3) }}{ }^{1}$ & $\mathrm{H}_{(\text {(corr-B3LYP-D3) }}{ }^{2}$ & $\mathrm{G}_{(\text {corr-B3LYP-D3) }}{ }^{3}$ & $\mathrm{E}_{(\text {solv-M062X) }}{ }^{4}$ & $\mathrm{IF}^{5}$ \\
\hline $\mathbf{2 a}$ & -1017.9716 & 0.5111 & 0.4289 & -1017.7698 & - \\
$\mathbf{2 b}$ & -1017.9710 & 0.5114 & 0.4302 & -1017.7688 & - \\
$\mathbf{B F}$ & -324.5539 & 0.0170 & -0.0119 & -324.5635 & - \\
$\mathbf{P h C H O}$ & -345.5815 & 0.1174 & 0.0797 & -345.5293 & - \\
TS-8a & -1688.1531 & 0.6502 & 0.5498 & -1687.9025 & -253.03 \\
TS-8b & -1688.1507 & 0.6502 & 0.5506 & -1687.8987 & -293.19 \\
\hline
\end{tabular}




\begin{tabular}{cccccc}
\hline TS-8c & -1688.1288 & 0.6504 & 0.5489 & -1687.8871 & -190.78 \\
TS-9a & -1688.1624 & 0.6500 & 0.5495 & -1687.9080 & -218.44 \\
TS-9b & -1688.1588 & 0.6500 & 0.5501 & -1687.9028 & -282.99 \\
TS-9c & -1688.1313 & 0.6500 & 0.5491 & -1687.8776 & -367.38 \\
TS-10a & -1688.1514 & 0.6506 & 0.5518 & -1687.9009 & -351.9 \\
TS-10b & -1688.1418 & 0.6505 & 0.5512 & -1687.8902 & -290.53 \\
TS-11a & -1688.1519 & 0.6504 & 0.5503 & -1687.8997 & -291.01 \\
TS-11b & -1688.1452 & 0.6504 & 0.5516 & -1687.8888 & -316.48 \\
\hline
\end{tabular}

\footnotetext{
${ }^{1}$ The electronic energy calculated by B3LYP-D3 in gas phase. ${ }^{2}$ The thermal correction to enthalpy calculated by B3LYP-D3 in gas phase. ${ }^{3}$ The thermal correction to Gibbs free energy calculated by B3LYP-D3 in gas phase. ${ }^{4}$ The electronic energy calculated by M062X in dichloromethane solvent. ${ }^{5}$ The B3LYP-D3 calculated imaginary frequencies for the transition states.
}

\section{B3LYP-D3 Geometries for All the Optimized Compounds and Transition State}

$\begin{array}{lrrr}\text { 2a } & \mathbf{E}=\mathbf{- 1 0 1 7 . 9 7 1 6 2 6 9 4} & \\ \mathrm{B} & 1.187478 & 0.218254 & 0.601295 \\ \mathrm{C} & 0.018071 & 1.116131 & 1.165712 \\ \mathrm{C} & 0.340267 & 2.491528 & 0.593425 \\ \mathrm{C} & 0.292097 & 2.850000 & -0.695359 \\ \mathrm{H} & 0.695251 & 3.223105 & 1.323216 \\ \mathrm{H} & -0.047593 & 2.122656 & -1.430805 \\ \mathrm{O} & 2.399827 & 0.049868 & 1.223828 \\ \mathrm{O} & 1.114669 & -0.429996 & -0.614567 \\ \mathrm{C} & 2.313657 & -1.243049 & -0.744880 \\ \mathrm{C} & 1.937517 & -2.658312 & -0.290954 \\ \mathrm{C} & 2.740079 & -1.246366 & -2.209032 \\ \mathrm{H} & 1.100881 & -3.016372 & -0.897974 \\ \mathrm{H} & 1.619261 & -2.659599 & 0.756129 \\ \mathrm{H} & 1.981493 & -1.755550 & -2.812103 \\ \mathrm{H} & 2.850785 & -0.230566 & -2.594212 \\ \mathrm{C} & 3.306501 & -0.522092 & 0.238973 \\ \mathrm{C} & 4.289917 & -1.440840 & 0.955540\end{array}$

$\begin{array}{lrrr}\mathrm{C} & 4.042417 & 0.657683 & -0.407865 \\ \mathrm{H} & 4.936799 & -1.952684 & 0.233870 \\ \mathrm{H} & 3.771765 & -2.190905 & 1.556911 \\ \mathrm{H} & 4.786398 & 0.320465 & -1.136889 \\ \mathrm{H} & 3.336773 & 1.330510 & -0.905509 \\ \mathrm{C} & 0.658641 & 4.210920 & -1.215050 \\ \mathrm{H} & 0.988308 & 4.876785 & -0.409113 \\ \mathrm{H} & 1.467698 & 4.153336 & -1.957109 \\ \mathrm{H} & -0.193146 & 4.687622 & -1.720126 \\ \mathrm{C} & 0.045307 & 1.134343 & 2.713525 \\ \mathrm{H} & -0.687452 & 1.847626 & 3.111708 \\ \mathrm{H} & -0.194105 & 0.147959 & 3.122299 \\ \mathrm{H} & 1.035051 & 1.422391 & 3.085805 \\ \mathrm{~B} & -1.301657 & 0.454718 & 0.626934 \\ \mathrm{O} & -1.812990 & -0.688619 & 1.205352 \\ \mathrm{O} & -2.012289 & 0.855546 & -0.476717 \\ \mathrm{C} & -3.192588 & 0.012152 & -0.567704 \\ \mathrm{C} & -4.341442 & 0.792911 & 0.081170\end{array}$




$\begin{array}{cccc}\mathrm{C} & -3.488806 & -0.246275 & -2.041604 \\ \mathrm{H} & -4.438839 & 1.760284 & -0.420588 \\ \mathrm{H} & -4.136625 & 0.979248 & 1.140009 \\ \mathrm{H} & -3.766789 & 0.692412 & -2.531305 \\ \mathrm{H} & -2.615226 & -0.650123 & -2.557641 \\ \mathrm{C} & -2.759911 & -1.253814 & 0.258049 \\ \mathrm{C} & -3.881598 & -1.937942 & 1.031982 \\ \mathrm{C} & -1.979096 & -2.276462 & -0.578169 \\ \mathrm{H} & -4.654515 & -2.306204 & 0.347619 \\ \mathrm{H} & -4.344382 & -1.259198 & 1.751678 \\ \mathrm{H} & -2.626892 & -2.792563 & -1.294472 \\ \mathrm{H} & -1.160212 & -1.790060 & -1.116079 \\ \mathrm{H} & 4.923915 & -0.850743 & 1.624673 \\ \mathrm{H} & 4.553559 & 1.225810 & 0.375088 \\ \mathrm{H} & 3.690411 & -1.777577 & -2.334704 \\ \mathrm{H} & 2.774638 & -3.354513 & -0.406078 \\ \mathrm{H} & -3.479093 & -2.792751 & 1.584704 \\ \mathrm{H} & -1.542634 & -3.020376 & 0.095659 \\ \mathrm{H} & -4.321622 & -0.950247 & -2.151963 \\ \mathrm{H} & -5.292871 & 0.257472 & -0.003572 \\ & & & \end{array}$

\section{PhCHO E = - $\mathbf{3 4 5 . 5 8 1 5 3 5 6 5 8}$}

$\begin{array}{lrrr}\mathrm{C} & -1.992547 & 0.470757 & -0.000023 \\ \mathrm{O} & -2.844959 & -0.397175 & -0.000033 \\ \mathrm{C} & -0.533559 & 0.218022 & -0.000003 \\ \mathrm{C} & -0.048539 & -1.099281 & 0.000005 \\ \mathrm{C} & 0.363737 & 1.294203 & 0.000004 \\ \mathrm{C} & 1.322662 & -1.333197 & 0.000017 \\ \mathrm{H} & -0.765989 & -1.914292 & -0.000001 \\ \mathrm{C} & 1.738444 & 1.058838 & 0.000016 \\ \mathrm{H} & -0.019017 & 2.312869 & -0.000003 \\ \mathrm{C} & 2.216167 & -0.254360 & 0.000022 \\ \mathrm{H} & 1.701712 & -2.351419 & 0.000022 \\ \mathrm{H} & 2.434973 & 1.892506 & 0.000019 \\ \mathrm{H} & 3.287060 & -0.439662 & 0.000031 \\ \mathrm{H} & -2.277250 & 1.547516 & -0.000034\end{array}$

\section{(R)-A $\mathbf{E}=\mathbf{- 2 5 8 2 . 2 2 1 9 5 1 9 2}$}

\begin{tabular}{|c|c|c|c|}
\hline $\mathrm{O}$ & 1.091567 & 0.118243 & 1.004959 \\
\hline $\mathrm{P}$ & 0.103720 & -1.023730 & 0.404541 \\
\hline $\mathrm{O}$ & -0.896496 & -0.158247 & -0.531756 \\
\hline $\mathrm{O}$ & -0.453670 & -1.856629 & 1.481563 \\
\hline $\mathrm{O}$ & 0.915083 & -1.751817 & -0.775800 \\
\hline $\mathrm{H}$ & 1.780882 & -2.084514 & -0.471554 \\
\hline $\mathrm{C}$ & 1.562393 & 1.119471 & 0.154477 \\
\hline$C$ & 0.706829 & 2.139443 & -0.220742 \\
\hline $\mathrm{C}$ & 2.924544 & 1.027864 & -0.241392 \\
\hline $\mathrm{C}$ & 1.222166 & 3.154073 & -1.100954 \\
\hline $\mathrm{C}$ & 3.436426 & 2.070894 & -0.981111 \\
\hline $\mathrm{C}$ & 2.616685 & 3.136447 & -1.435257 \\
\hline $\mathrm{H}$ & 4.482167 & 2.054415 & -1.273265 \\
\hline $\mathrm{C}$ & -1.482268 & 0.977465 & 0.042542 \\
\hline $\mathrm{C}$ & -0.712545 & 2.116031 & 0.234237 \\
\hline $\mathrm{C}$ & -2.868227 & 0.895658 & 0.338246 \\
\hline $\mathrm{C}$ & -1.326164 & 3.235085 & 0.895855 \\
\hline $\mathrm{C}$ & -3.468201 & 2.018032 & 0.867595 \\
\hline $\mathrm{C}$ & -2.728368 & 3.184560 & 1.187048 \\
\hline $\mathrm{H}$ & -4.533351 & 1.996164 & 1.079218 \\
\hline $\mathrm{C}$ & -3.632885 & -0.363212 & 0.080349 \\
\hline $\mathrm{C}$ & -4.059568 & -1.161277 & 1.164311 \\
\hline $\mathrm{C}$ & -3.929375 & -0.746262 & -1.247247 \\
\hline $\mathrm{C}$ & -4.810055 & -2.311159 & 0.891569 \\
\hline $\mathrm{C}$ & -4.670764 & -1.909539 & -1.462712 \\
\hline $\mathrm{C}$ & -5.126553 & -2.704851 & -0.407852 \\
\hline $\mathrm{H}$ & -5.151092 & -2.926597 & 1.720188 \\
\hline $\mathrm{H}$ & -4.900672 & -2.202578 & -2.483889 \\
\hline $\mathrm{C}$ & 3.694584 & -0.215331 & 0.076002 \\
\hline $\mathrm{C}$ & 4.002460 & -1.131224 & -0.966921 \\
\hline $\mathrm{C}$ & 4.034409 & -0.524816 & 1.410505 \\
\hline$C$ & 4.626219 & -2.338669 & -0.632957 \\
\hline $\mathrm{C}$ & 4.670115 & -1.741760 & 1.683171 \\
\hline $\mathrm{C}$ & 4.965013 & -2.667676 & 0.684123 \\
\hline $\mathrm{H}$ & 4.863387 & -3.041290 & -1.426129 \\
\hline
\end{tabular}




\begin{tabular}{|c|c|c|c|c|c|c|c|}
\hline $\mathrm{H}$ & 4.934906 & -1.979140 & 2.710539 & $\mathrm{H}$ & 4.888167 & 1.729553 & 3.912036 \\
\hline $\mathrm{C}$ & -3.743041 & -0.810189 & 2.616427 & $\mathrm{H}$ & 5.713438 & 0.207992 & 3.531435 \\
\hline $\mathrm{C}$ & -3.158826 & -1.999583 & 3.399836 & $\mathrm{H}$ & 1.985012 & -0.609450 & 3.283244 \\
\hline $\mathrm{C}$ & -4.991011 & -0.249659 & 3.325363 & $\mathrm{H}$ & 3.466110 & -1.026037 & 4.169603 \\
\hline $\mathrm{H}$ & -2.978996 & -0.026917 & 2.617621 & $\mathrm{H}$ & 2.683020 & 0.533892 & 4.447406 \\
\hline $\mathrm{H}$ & -2.274933 & -2.395402 & 2.895245 & $\mathrm{C}$ & 3.722567 & -0.828070 & -2.440537 \\
\hline $\mathrm{H}$ & -2.866553 & -1.672164 & 4.405064 & $\mathrm{C}$ & 3.122353 & -2.010636 & -3.222890 \\
\hline $\mathrm{H}$ & -3.890531 & -2.807286 & 3.520836 & $\mathrm{C}$ & 5.016744 & -0.347378 & -3.128441 \\
\hline $\mathrm{H}$ & -5.382355 & 0.637237 & 2.814572 & $\mathrm{H}$ & 2.993808 & -0.014552 & -2.489345 \\
\hline $\mathrm{H}$ & -5.792228 & -0.998385 & 3.349891 & $\mathrm{H}$ & 2.191201 & -2.363643 & -2.772049 \\
\hline $\mathrm{H}$ & -4.756238 & 0.028363 & 4.359981 & $\mathrm{H}$ & 2.895663 & -1.693481 & -4.247182 \\
\hline $\mathrm{C}$ & -3.482987 & 0.075440 & -2.453500 & $\mathrm{H}$ & 3.816808 & -2.855573 & -3.293664 \\
\hline $\mathrm{C}$ & -4.692166 & 0.621553 & -3.235570 & $\mathrm{H}$ & 5.448828 & 0.519868 & -2.618869 \\
\hline $\mathrm{C}$ & -2.540171 & -0.727785 & -3.368435 & $\mathrm{H}$ & 5.772280 & -1.142414 & -3.124353 \\
\hline $\mathrm{H}$ & -2.921569 & 0.942817 & -2.093459 & $\mathrm{H}$ & 4.820766 & -0.069401 & -4.170785 \\
\hline $\mathrm{H}$ & -5.340773 & 1.222373 & -2.588029 & $\mathrm{C}$ & 5.627909 & -3.995679 & 1.015275 \\
\hline $\mathrm{H}$ & -4.358891 & 1.252154 & -4.068750 & $\mathrm{C}$ & 6.995271 & -4.137821 & 0.322031 \\
\hline $\mathrm{H}$ & -5.298152 & -0.191018 & -3.653147 & $\mathrm{C}$ & 4.707324 & -5.181547 & 0.671766 \\
\hline $\mathrm{H}$ & -1.669212 & -1.083718 & -2.810753 & $\mathrm{H}$ & 5.801785 & -4.013421 & 2.099720 \\
\hline $\mathrm{H}$ & -3.049686 & -1.597109 & -3.800842 & $\mathrm{H}$ & 7.659647 & -3.307253 & 0.584252 \\
\hline $\mathrm{H}$ & -2.187997 & -0.101876 & -4.197556 & $\mathrm{H}$ & 7.482076 & -5.074897 & 0.616770 \\
\hline $\mathrm{C}$ & -5.933197 & -3.967976 & -0.667911 & $\mathrm{H}$ & 6.886434 & -4.147883 & -0.769004 \\
\hline $\mathrm{C}$ & -5.111937 & -5.008290 & -1.451694 & $\mathrm{H}$ & 3.745157 & -5.097602 & 1.188794 \\
\hline $\mathrm{C}$ & -7.259551 & -3.657546 & -1.385429 & $\mathrm{H}$ & 4.506647 & -5.225383 & -0.405432 \\
\hline $\mathrm{H}$ & -6.179221 & -4.407230 & 0.308642 & $\mathrm{H}$ & 5.172578 & -6.130408 & 0.963550 \\
\hline $\mathrm{H}$ & -4.180904 & -5.250002 & -0.927870 & $\mathrm{C}$ & -3.348458 & 4.296276 & 1.818728 \\
\hline $\mathrm{H}$ & -5.684177 & -5.934245 & -1.586125 & $\mathrm{C}$ & -2.616317 & 5.402390 & 2.182663 \\
\hline $\mathrm{H}$ & -4.846698 & -4.631156 & -2.446602 & $\mathrm{H}$ & -3.100221 & 6.243187 & 2.671743 \\
\hline $\mathrm{H}$ & -7.857487 & -2.937988 & -0.815384 & $\mathrm{C}$ & -1.223243 & 5.436055 & 1.936398 \\
\hline $\mathrm{H}$ & -7.079636 & -3.229278 & -2.378710 & $\mathrm{H}$ & -0.641740 & 6.298660 & 2.249895 \\
\hline $\mathrm{H}$ & -7.852573 & -4.570528 & -1.517962 & $\mathrm{C}$ & -0.595302 & 4.382355 & 1.310319 \\
\hline $\mathrm{C}$ & 3.762354 & 0.437262 & 2.562379 & $\mathrm{H}$ & 0.473904 & 4.418897 & 1.136940 \\
\hline $\mathrm{C}$ & 5.084956 & 1.003733 & 3.114131 & $\mathrm{C}$ & 0.406590 & 4.161480 & -1.685184 \\
\hline $\mathrm{C}$ & 2.922876 & -0.210015 & 3.678566 & $\mathrm{H}$ & -0.657671 & 4.163494 & -1.482594 \\
\hline $\mathrm{H}$ & 3.189058 & 1.283927 & 2.174140 & $\mathrm{C}$ & 0.947415 & 5.125601 & -2.506889 \\
\hline $\mathrm{H}$ & 5.658964 & 1.505742 & 2.327009 & $\mathrm{H}$ & 0.301615 & 5.881991 & -2.944191 \\
\hline
\end{tabular}




$\begin{array}{lrrr}\mathrm{C} & 2.332889 & 5.136354 & -2.792737 \\ \mathrm{C} & 3.145449 & 4.156579 & -2.271253 \\ \mathrm{H} & -4.415149 & 4.246746 & 2.023491 \\ \mathrm{H} & 2.747526 & 5.907228 & -3.436220 \\ \mathrm{H} & 4.207046 & 4.135766 & -2.505745\end{array}$

\section{TS-3 E = -1363.56288962}

\begin{tabular}{|c|c|c|c|}
\hline $\mathrm{C}$ & -0.803467 & 0.172261 & 1.665760 \\
\hline $\mathrm{C}$ & 0.307491 & -0.376835 & 2.400496 \\
\hline $\mathrm{C}$ & 1.653298 & -0.201999 & 2.144421 \\
\hline $\mathrm{O}$ & -1.904543 & -0.542159 & -0.646138 \\
\hline B & -0.909342 & -0.976711 & 0.272447 \\
\hline $\mathrm{O}$ & 0.470637 & -0.868692 & -0.331194 \\
\hline $\mathrm{O}$ & -1.197865 & -2.313972 & 0.690230 \\
\hline $\mathrm{C}$ & 1.473961 & -1.421619 & 0.266606 \\
\hline $\mathrm{C}$ & 2.818713 & -1.169903 & -0.263489 \\
\hline $\mathrm{C}$ & 3.089429 & 0.001291 & -0.992247 \\
\hline $\mathrm{C}$ & 3.841918 & -2.104372 & -0.046137 \\
\hline $\mathrm{C}$ & 5.119153 & -1.878446 & -0.560619 \\
\hline $\mathrm{C}$ & 4.364681 & 0.223151 & -1.498858 \\
\hline $\mathrm{C}$ & 5.381976 & -0.716143 & -1.287570 \\
\hline $\mathrm{H}$ & 0.050288 & -1.142457 & 3.138238 \\
\hline $\mathrm{H}$ & 1.948683 & 0.654464 & 1.545331 \\
\hline $\mathrm{H}$ & 1.294221 & -2.359881 & 0.795949 \\
\hline $\mathrm{H}$ & 2.292986 & 0.726186 & -1.117951 \\
\hline $\mathrm{H}$ & 3.630371 & -3.011452 & 0.514844 \\
\hline $\mathrm{H}$ & 5.906022 & -2.609067 & -0.395297 \\
\hline $\mathrm{H}$ & 4.572933 & 1.131170 & -2.058468 \\
\hline $\mathrm{H}$ & 6.376824 & -0.538881 & -1.687092 \\
\hline $\mathrm{C}$ & -2.153437 & 0.046660 & 2.391864 \\
\hline $\mathrm{C}$ & -2.445655 & -1.732473 & -1.245452 \\
\hline $\mathrm{C}$ & -1.591127 & -2.074315 & -2.478108 \\
\hline $\mathrm{C}$ & -3.878926 & -1.436512 & -1.683769 \\
\hline $\mathrm{H}$ & -1.984693 & -2.940851 & -3.020533 \\
\hline $\mathrm{H}$ & -0.553613 & -2.274680 & -2.199125 \\
\hline $\mathrm{H}$ & -4.367103 & -2.337970 & -2.072061 \\
\hline
\end{tabular}

\begin{tabular}{|c|c|c|c|}
\hline $\mathrm{H}$ & -4.472764 & -1.041931 & -0.856592 \\
\hline $\mathrm{C}$ & -2.312977 & -2.799300 & -0.082595 \\
\hline C & -3.542488 & -2.845655 & 0.834732 \\
\hline $\mathrm{C}$ & -1.984436 & -4.216538 & -0.558019 \\
\hline $\mathrm{H}$ & -4.402070 & -3.307812 & 0.336709 \\
\hline $\mathrm{H}$ & -3.827341 & -1.845736 & 1.166745 \\
\hline $\mathrm{H}$ & -2.771782 & -4.607124 & -1.213523 \\
\hline $\mathrm{H}$ & -1.034185 & -4.245309 & -1.096168 \\
\hline $\mathrm{H}$ & -1.592834 & -1.211995 & -3.152511 \\
\hline $\mathrm{H}$ & -3.869332 & -0.683502 & -2.478922 \\
\hline $\mathrm{H}$ & -1.900906 & -4.882016 & 0.307904 \\
\hline $\mathrm{H}$ & -3.295565 & -3.438827 & 1.721352 \\
\hline $\mathrm{H}$ & -2.971780 & 0.259835 & 1.700656 \\
\hline $\mathrm{H}$ & -2.291750 & -0.962055 & 2.794334 \\
\hline $\mathrm{H}$ & -2.223735 & 0.764265 & 3.218479 \\
\hline B & -0.646851 & 1.527268 & 0.902625 \\
\hline $\mathrm{O}$ & 0.494877 & 1.966084 & 0.257690 \\
\hline $\mathrm{O}$ & -1.704352 & 2.388533 & 0.742920 \\
\hline $\mathrm{C}$ & -1.223767 & 3.553587 & 0.023334 \\
\hline $\mathrm{C}$ & -2.323825 & 4.018220 & -0.925950 \\
\hline $\mathrm{C}$ & -0.924748 & 4.631168 & 1.071913 \\
\hline $\mathrm{H}$ & -3.179767 & 4.380576 & -0.347746 \\
\hline $\mathrm{H}$ & -2.667770 & 3.198899 & -1.560395 \\
\hline $\mathrm{H}$ & -1.828088 & 4.809531 & 1.662959 \\
\hline $\mathrm{H}$ & -0.132741 & 4.302769 & 1.752305 \\
\hline $\mathrm{C}$ & 0.068703 & 2.997921 & -0.677684 \\
\hline $\mathrm{C}$ & -0.231880 & 2.289770 & -2.006169 \\
\hline $\mathrm{C}$ & 1.203508 & 4.002016 & -0.843844 \\
\hline $\mathrm{H}$ & -0.546618 & 3.002352 & -2.775787 \\
\hline $\mathrm{H}$ & -1.004974 & 1.526998 & -1.874430 \\
\hline $\mathrm{H}$ & 0.891405 & 4.835413 & -1.483379 \\
\hline $\mathrm{H}$ & 1.532197 & 4.401315 & 0.118222 \\
\hline $\mathrm{H}$ & 0.673485 & 1.784888 & -2.356449 \\
\hline $\mathrm{H}$ & 2.061317 & 3.513079 & -1.317601 \\
\hline $\mathrm{H}$ & -0.620044 & 5.574704 & 0.606931 \\
\hline $\mathrm{H}$ & -1.968475 & 4.836905 & -1.562377 \\
\hline
\end{tabular}




$$
\begin{array}{llll}
\mathrm{C} & 2.721435 & -0.818151 & 3.008732 \\
\mathrm{H} & 2.350024 & -1.699798 & 3.544322 \\
\mathrm{H} & 3.593990 & -1.117323 & 2.416067 \\
\mathrm{H} & 3.078924 & -0.098530 & 3.757545
\end{array}
$$

\section{TS-4 E = -1363.55777155}

$\begin{array}{llll}\text { C } & 0.223504 & -1.092819 & -0.438550\end{array}$

$\begin{array}{llll}\text { C } & -0.475998 & -1.620553 & 0.693121\end{array}$

$\begin{array}{llll}\text { C } & -1.784378 & -2.082008 & 0.698751\end{array}$

$\begin{array}{llll}\text { O } & 0.384327 & 1.468823 & -1.161865\end{array}$

B $\quad-0.284072 \quad 0.643473 \quad-0.223229$

$\begin{array}{llll}\text { O } & -1.757632 & 0.471504 & -0.537840\end{array}$

$\begin{array}{llll}\text { O } & -0.106777 & 1.172042 & 1.092479\end{array}$

$\begin{array}{llll}\text { C } & -2.526701 & -0.029136 & 0.383729\end{array}$

$\begin{array}{llll}\text { C } & -3.920905 & -0.327735 & 0.020425\end{array}$

$\begin{array}{llll}\text { C } & -4.290954 & -0.512179 & -1.322343\end{array}$

$\begin{array}{llll}\text { C } & -4.900098 & -0.413065 & 1.021939\end{array}$

$\begin{array}{llll}\text { C } & -6.225821 & -0.692213 & 0.689480\end{array}$

$\begin{array}{llll}\text { C } & -5.613735 & -0.791043 & -1.649798\end{array}$

$\begin{array}{llll}\text { C } & -6.584411 & -0.885817 & -0.645596\end{array}$

$\mathrm{H} \quad-0.013561 \quad-1.432528 \quad 1.662768$

$\mathrm{H} \quad-2.207091 \quad-2.433602 \quad-0.239134$

$\mathrm{H} \quad-2.322040 \quad 0.263355 \quad 1.415564$

$\mathrm{H} \quad-3.531132 \quad-0.423573 \quad-2.092183$

$\mathrm{H} \quad-4.621071 \quad-0.246423 \quad 2.059280$

$\begin{array}{llll}\mathrm{H} & -6.977720 & -0.752835 & 1.471397\end{array}$

H $\quad-5.893639 \quad-0.932329 \quad-2.690186$

H $\quad-7.616656 \quad-1.103586 \quad-0.905716$

$\begin{array}{llll}\text { C } & -0.197100 & -1.539222 & -1.847721\end{array}$

$\begin{array}{llll}\text { C } & 0.422094 & 2.781934 & -0.570945\end{array}$

$\begin{array}{llll}\text { C } & -0.869186 & 3.518183 & -0.969178\end{array}$

$\begin{array}{llll}\text { C } & 1.624562 & 3.532059 & -1.141431\end{array}$

$\begin{array}{llll}\mathrm{H} & -0.879608 & 4.550232 & -0.601939\end{array}$

$\begin{array}{llll}\mathrm{H} & -1.755230 & 3.001442 & -0.592373\end{array}$

$\mathrm{H} \quad 1.728801 \quad 4.518505 \quad-0.674800$

$\begin{array}{llll}\mathrm{H} & 2.552017 & 2.975970 & -0.992517\end{array}$ $\begin{array}{llll}\text { C } & 0.508194 & 2.469132 & 0.980643\end{array}$

$\begin{array}{llll}\text { C } & 1.946756 & 2.344017 & 1.493660\end{array}$

$\begin{array}{llll}\text { C } & -0.265291 & 3.447922 & 1.867312\end{array}$

H $\quad 2.462989 \quad 3.310499 \quad 1.505509$

$\begin{array}{llll}\mathrm{H} & 2.508494 & 1.637974 & 0.884014\end{array}$

$\mathrm{H} \quad 0.128098 \quad 4.466708 \quad 1.769078$

$\mathrm{H} \quad-1.328952 \quad 3.458400 \quad 1.617934$

$\mathrm{H} \quad-0.935243 \quad 3.535768 \quad-2.061531$

$\mathrm{H} \quad 1.488446 \quad 3.674479 \quad-2.218425$

$\begin{array}{llll}\mathrm{H} & -0.167930 & 3.144869 & 2.915291\end{array}$

$\begin{array}{llll}\mathrm{H} & 1.923862 & 1.943694 & 2.511909\end{array}$

H $\quad 0.251170 \quad-0.874932 \quad-2.590797$

$\mathrm{H} \quad 0.156940 \quad-2.556718 \quad-2.055606$

H $\quad-1.278999 \quad-1.514361 \quad-1.994135$

$\begin{array}{llll}\text { B } & 1.778793 & -1.051146 & -0.271298\end{array}$

$\begin{array}{llll}\text { O } & 2.623740 & -1.327105 & -1.319123\end{array}$

O $\quad 2.445136 \quad-0.845621 \quad 0.915568$

$\begin{array}{llll}\text { C } & 3.828268 & -1.243716 & 0.704743\end{array}$

$\begin{array}{llll}\text { C } & 4.737909 & -0.333928 & 1.524494\end{array}$

$\begin{array}{llll}\text { C } & 3.940576 & -2.690052 & 1.203658\end{array}$

H $\quad 4.524058 \quad-0.464872 \quad 2.589923$

$\mathrm{H} \quad 4.588746 \quad 0.718772 \quad 1.279336$

$\mathrm{H} \quad 3.621438 \quad-2.727484 \quad 2.249641$

$\mathrm{H} \quad 3.289261 \quad-3.354192 \quad 0.626752$

$\begin{array}{llll}\text { C } & 3.983287 & -1.112589 & -0.857361\end{array}$

$\begin{array}{llll}\text { C } & 4.388043 & 0.292068 & -1.317675\end{array}$

$\begin{array}{llll}\text { C } & 4.886381 & -2.158954 & -1.504711\end{array}$

$\begin{array}{llll}\mathrm{H} & 5.425105 & 0.522297 & -1.052232\end{array}$

$\begin{array}{llll}\mathrm{H} & 3.732714 & 1.049715 & -0.881494\end{array}$

H $\quad 5.909025 \quad-2.079650 \quad-1.118516$

$\mathrm{H} \quad 4.520518 \quad-3.172104 \quad-1.324845$

$\mathrm{H} \quad 4.282599 \quad 0.349997 \quad-2.404930$

H $\quad 4.916066 \quad-1.998617 \quad-2.586956$

$\mathrm{H} \quad 4.967684 \quad-3.063557 \quad 1.138567$

H $\quad 5.789873 \quad-0.588459 \quad 1.351663$

$\begin{array}{llll}\text { C } & -2.435238 & -2.621387 & 1.946015\end{array}$

\section{SI-41}




$\begin{array}{llll}\mathrm{H} & -2.025822 & -2.151299 & 2.847892 \\ \mathrm{H} & -3.518804 & -2.459111 & 1.931821 \\ \mathrm{H} & -2.276909 & -3.704616 & 2.032894\end{array}$

\section{TS-5 E = -3945.85539349}

\begin{tabular}{|c|c|c|c|}
\hline $\mathrm{C}$ & 2.881099 & 3.279783 & 0.180507 \\
\hline $\mathrm{C}$ & 3.158774 & 3.750212 & -1.123816 \\
\hline $\mathrm{C}$ & 2.461449 & 4.862440 & -1.602562 \\
\hline $\mathrm{C}$ & 1.508084 & 5.529804 & -0.829218 \\
\hline $\mathrm{C}$ & 1.242548 & 5.036044 & 0.447961 \\
\hline $\mathrm{C}$ & 1.898001 & 3.912707 & 0.969061 \\
\hline $\mathrm{O}$ & 2.316133 & 0.551137 & -0.441224 \\
\hline $\mathrm{P}$ & 1.116415 & -0.232603 & 0.348218 \\
\hline $\mathrm{C}$ & 3.456170 & 0.802597 & 0.329088 \\
\hline $\mathrm{O}$ & 0.153636 & -0.644680 & -0.819273 \\
\hline $\mathrm{O}$ & 1.837821 & -1.604140 & 0.823062 \\
\hline $\mathrm{C}$ & 4.304875 & -0.252998 & 0.624278 \\
\hline $\mathrm{C}$ & 3.697686 & 2.145753 & 0.722532 \\
\hline $\mathrm{C}$ & 2.845333 & -2.198850 & 0.069028 \\
\hline $\mathrm{C}$ & 4.080569 & -1.579749 & -0.019134 \\
\hline $\mathrm{C}$ & 4.787982 & 2.378840 & 1.533122 \\
\hline $\mathrm{C}$ & 5.385132 & -0.011541 & 1.539348 \\
\hline $\mathrm{C}$ & 5.620939 & 1.326666 & 1.994097 \\
\hline $\mathrm{C}$ & 2.543099 & -3.458757 & -0.512082 \\
\hline $\mathrm{C}$ & 5.098237 & -2.217663 & -0.810233 \\
\hline $\mathrm{C}$ & 3.561197 & -4.110227 & -1.173682 \\
\hline $\mathrm{C}$ & 4.836180 & -3.516728 & -1.357041 \\
\hline $\mathrm{C}$ & 1.161519 & -4.028244 & -0.427527 \\
\hline $\mathrm{C}$ & 0.323467 & -3.995235 & -1.567422 \\
\hline $\mathrm{C}$ & -0.950571 & -4.563510 & -1.479339 \\
\hline $\mathrm{C}$ & -1.423676 & -5.154563 & -0.303559 \\
\hline $\mathrm{C}$ & -0.583639 & -5.155068 & 0.809815 \\
\hline $\mathrm{C}$ & 0.700437 & -4.598415 & 0.776160 \\
\hline $\mathrm{O}$ & 0.546475 & 0.476150 & 1.516856 \\
\hline $\mathrm{H}$ & 2.675547 & 5.222347 & -2.605321 \\
\hline $\mathrm{H}$ & 0.496130 & 5.540341 & 1.055764 \\
\hline
\end{tabular}

\begin{tabular}{|c|c|c|c|}
\hline $\mathrm{H}$ & 5.018001 & 3.399013 & 1.827767 \\
\hline $\mathrm{H}$ & 3.373138 & -5.091760 & -1.599309 \\
\hline $\mathrm{H}$ & -1.590266 & -4.553585 & -2.357184 \\
\hline $\mathrm{H}$ & -0.940072 & -5.603961 & 1.734205 \\
\hline $\mathrm{H}$ & -0.659640 & -0.016827 & -0.956985 \\
\hline c & -3.963713 & -0.715012 & -0.939683 \\
\hline$C$ & -3.129288 & -1.470014 & -0.046362 \\
\hline $\mathrm{C}$ & -3.201063 & -1.500559 & 1.341802 \\
\hline $\mathrm{O}$ & -3.862918 & 1.899169 & -1.531046 \\
\hline B & -3.202653 & 0.916365 & -0.748075 \\
\hline $\mathrm{O}$ & -3.323312 & 1.183033 & 0.698043 \\
\hline $\mathrm{O}$ & -1.806193 & 0.890848 & -1.201159 \\
\hline C & -2.583736 & 0.522562 & 1.555617 \\
\hline $\mathrm{C}$ & -2.858685 & 0.733329 & 2.986113 \\
\hline $\mathrm{C}$ & -4.145910 & 1.066596 & 3.436146 \\
\hline $\mathrm{C}$ & -1.807703 & 0.596776 & 3.906742 \\
\hline $\mathrm{C}$ & -2.045142 & 0.804519 & 5.266265 \\
\hline $\mathrm{C}$ & -4.374615 & 1.270395 & 4.792951 \\
\hline $\mathrm{C}$ & -3.325046 & 1.141864 & 5.710856 \\
\hline $\mathrm{H}$ & -2.241292 & -1.927441 & -0.490887 \\
\hline $\mathrm{H}$ & -4.162797 & -1.279654 & 1.796583 \\
\hline $\mathrm{H}$ & -1.545248 & 0.345900 & 1.293475 \\
\hline $\mathrm{H}$ & -4.947270 & 1.133328 & 2.709284 \\
\hline $\mathrm{H}$ & -0.812977 & 0.356610 & 3.543173 \\
\hline $\mathrm{H}$ & -1.228783 & 0.706552 & 5.976708 \\
\hline $\mathrm{H}$ & -5.371846 & 1.527030 & 5.141153 \\
\hline $\mathrm{H}$ & -3.507757 & 1.302879 & 6.770122 \\
\hline $\mathrm{C}$ & 1.564970 & -4.647033 & 2.032494 \\
\hline $\mathrm{C}$ & 1.843965 & -6.099014 & 2.463441 \\
\hline $\mathrm{C}$ & 0.946859 & -3.830819 & 3.182522 \\
\hline $\mathrm{H}$ & 2.532816 & -4.193030 & 1.798492 \\
\hline $\mathrm{H}$ & 2.311201 & -6.667964 & 1.651703 \\
\hline $\mathrm{H}$ & 2.516918 & -6.119629 & 3.329007 \\
\hline $\mathrm{H}$ & 0.920724 & -6.617793 & 2.746965 \\
\hline $\mathrm{H}$ & 0.817070 & -2.785224 & 2.890154 \\
\hline $\mathrm{H}$ & -0.031661 & -4.230096 & 3.474771 \\
\hline
\end{tabular}




\begin{tabular}{|c|c|c|c|c|c|c|c|}
\hline $\mathrm{H}$ & 1.597727 & -3.862698 & 4.064687 & $\mathrm{H}$ & -0.171711 & 2.910976 & 3.590395 \\
\hline $\mathrm{C}$ & 0.785161 & -3.399134 & -2.895972 & $\mathrm{H}$ & -0.408407 & 4.384696 & 2.648011 \\
\hline $\mathrm{C}$ & -0.263858 & -2.479980 & -3.542663 & $\mathrm{H}$ & 3.344898 & 4.259795 & 3.319348 \\
\hline $\mathrm{C}$ & 1.195451 & -4.516098 & -3.875499 & $\mathrm{H}$ & 1.936500 & 5.333198 & 3.365179 \\
\hline $\mathrm{H}$ & 1.668094 & -2.782871 & -2.701341 & $\mathrm{H}$ & 2.015173 & 3.926967 & 4.445463 \\
\hline $\mathrm{H}$ & -0.565012 & -1.696279 & -2.846499 & $\mathrm{C}$ & 0.805722 & 6.777726 & -1.345310 \\
\hline $\mathrm{H}$ & 0.155555 & -2.003895 & -4.437049 & $\mathrm{C}$ & 0.082094 & 6.543269 & -2.683283 \\
\hline $\mathrm{H}$ & -1.159742 & -3.027997 & -3.856368 & $\mathrm{C}$ & 1.797098 & 7.951490 & -1.461650 \\
\hline $\mathrm{H}$ & 1.990602 & -5.144058 & -3.460754 & $\mathrm{H}$ & 0.045031 & 7.056374 & -0.603144 \\
\hline $\mathrm{H}$ & 0.340559 & -5.166033 & -4.098908 & $\mathrm{H}$ & -0.661071 & 5.743696 & -2.599853 \\
\hline $\mathrm{H}$ & 1.554935 & -4.090710 & -4.820269 & $\mathrm{H}$ & -0.434800 & 7.454657 & -3.006212 \\
\hline $\mathrm{C}$ & -2.794388 & -5.814249 & -0.250522 & $\mathrm{H}$ & 0.787040 & 6.264157 & -3.475165 \\
\hline $\mathrm{C}$ & -2.837107 & -7.059481 & -1.156822 & $\mathrm{H}$ & 2.288427 & 8.147750 & -0.502459 \\
\hline $\mathrm{C}$ & -3.932878 & -4.840966 & -0.604108 & $\mathrm{H}$ & 2.578946 & 7.728117 & -2.197539 \\
\hline $\mathrm{H}$ & -2.956185 & -6.150629 & 0.783060 & $\mathrm{H}$ & 1.284297 & 8.866863 & -1.781044 \\
\hline $\mathrm{H}$ & -2.048246 & -7.770518 & -0.887942 & $\mathrm{C}$ & 6.691645 & 1.575298 & 2.894150 \\
\hline $\mathrm{H}$ & -3.804734 & -7.568869 & -1.073750 & $\mathrm{H}$ & 6.865149 & 2.596875 & 3.223789 \\
\hline $\mathrm{H}$ & -2.692610 & -6.781848 & -2.207809 & $\mathrm{C}$ & 7.484196 & 0.548009 & 3.351921 \\
\hline $\mathrm{H}$ & -3.946482 & -3.981498 & 0.072874 & $\mathrm{H}$ & 8.295905 & 0.749923 & 4.045291 \\
\hline $\mathrm{H}$ & -3.826425 & -4.456107 & -1.625280 & $\mathrm{C}$ & 7.232264 & -0.778736 & 2.928904 \\
\hline $\mathrm{H}$ & -4.904707 & -5.344768 & -0.538897 & $\mathrm{C}$ & 6.211808 & -1.051559 & 2.045192 \\
\hline $\mathrm{C}$ & 4.188145 & 3.076293 & -2.028765 & $\mathrm{H}$ & 6.023462 & -2.072818 & 1.734025 \\
\hline $\mathrm{C}$ & 5.225059 & 4.073105 & -2.576722 & $\mathrm{C}$ & 5.845511 & -4.171410 & -2.113146 \\
\hline $\mathrm{C}$ & 3.495958 & 2.305945 & -3.169399 & $\mathrm{C}$ & 7.055625 & -3.563674 & -2.354580 \\
\hline $\mathrm{H}$ & 4.741448 & 2.344028 & -1.433398 & $\mathrm{H}$ & 7.817353 & -4.071621 & -2.939494 \\
\hline $\mathrm{H}$ & 5.713975 & 4.620730 & -1.763265 & $\mathrm{C}$ & 7.299929 & -2.262691 & -1.855054 \\
\hline $\mathrm{H}$ & 5.997131 & 3.542250 & -3.146211 & $\mathrm{C}$ & 6.348705 & -1.607431 & -1.104845 \\
\hline $\mathrm{H}$ & 4.768988 & 4.808901 & -3.249042 & $\mathrm{H}$ & 6.550053 & -0.608267 & -0.737402 \\
\hline $\mathrm{H}$ & 2.827024 & 1.538368 & -2.769491 & $\mathrm{H}$ & 7.846403 & -1.590588 & 3.308766 \\
\hline $\mathrm{H}$ & 2.902968 & 2.983085 & -3.796202 & $\mathrm{H}$ & 5.633885 & -5.160683 & -2.511924 \\
\hline $\mathrm{H}$ & 4.239002 & 1.815069 & -3.809734 & $\mathrm{H}$ & 8.245462 & -1.772351 & -2.069906 \\
\hline $\mathrm{C}$ & 1.552010 & 3.424625 & 2.372151 & $\mathrm{C}$ & -3.818974 & -1.105194 & -2.418819 \\
\hline $\mathrm{C}$ & 0.035446 & 3.382396 & 2.624274 & $\mathrm{C}$ & -2.861969 & 2.860329 & -1.922252 \\
\hline $\mathrm{C}$ & 2.256223 & 4.286218 & 3.437618 & $\mathrm{C}$ & -2.661616 & 3.863819 & -0.773165 \\
\hline $\mathrm{H}$ & 1.914095 & 2.397444 & 2.468679 & $\mathrm{C}$ & -3.364902 & 3.591549 & -3.164279 \\
\hline $\mathrm{H}$ & -0.469276 & 2.792791 & 1.855049 & $\mathrm{H}$ & -1.968314 & 4.663493 & -1.052996 \\
\hline
\end{tabular}




$\begin{array}{rrrrrrrr}\mathrm{H} & -2.279875 & 3.377103 & 0.126631 & \mathrm{H} & -9.398542 & -1.194523 & -0.286750 \\ \mathrm{H} & -2.594338 & 4.261296 & -3.563695 & \mathrm{H} & -9.383989 & 1.321808 & -1.142336 \\ \mathrm{H} & -3.662547 & 2.888460 & -3.945151 & \mathrm{C} & -2.248529 & -2.354079 & 2.140445 \\ \mathrm{C} & -1.593641 & 1.962537 & -2.167097 & \mathrm{H} & -1.284693 & -2.459140 & 1.634286 \\ \mathrm{C} & -1.551513 & 1.342315 & -3.566901 & \mathrm{H} & -2.073756 & -1.934014 & 3.136158 \\ \mathrm{C} & -0.253398 & 2.619495 & -1.857789 & \mathrm{H} & -2.654514 & -3.364548 & 2.278394\end{array}$

$\mathrm{H} \quad-1.300303 \quad 2.096881 \quad-4.319641$

$\mathrm{H} \quad-2.506151 \quad 0.886114 \quad-3.832156$

$\begin{array}{llll}\mathrm{H} & -0.083430 & 3.483333 & -2.506079\end{array}$

$\mathrm{H} \quad-0.184070 \quad 2.950766 \quad-0.821326$

$\mathrm{H} \quad-3.630669 \quad 4.309340 \quad-0.527931$

$\mathrm{H} \quad-4.239876 \quad 4.195110 \quad-2.902304$

$\begin{array}{llll}\mathrm{H} & 0.560844 & 1.911733 & -2.035274\end{array}$

$\begin{array}{llll}\mathrm{H} & -0.779922 & 0.566650 & -3.593274\end{array}$

$\mathrm{H} \quad-4.268843 \quad-0.341470 \quad-3.056754$

$\mathrm{H} \quad-2.770240 \quad-1.226404 \quad-2.702090$

$\mathrm{H} \quad-4.337241 \quad-2.050622 \quad-2.620510$

B $\quad-5.441404 \quad-0.377906 \quad-0.551978$

$\begin{array}{llll}\text { O } & -5.911111 & -0.125203 & 0.720626\end{array}$

O $\quad-6.411873 \quad-0.232150 \quad-1.512406$

$\begin{array}{llll}\text { C } & -7.668315 & 0.028922 & -0.831788\end{array}$

$\begin{array}{llll}\text { C } & -8.471621 & 1.018891 & -1.668893\end{array}$

$\begin{array}{llll}\text { C } & -8.402970 & -1.312400 & -0.727294\end{array}$

$\mathrm{H} \quad-8.761579 \quad 0.550908 \quad-2.615021$

$\mathrm{H} \quad-7.882945 \quad 1.909183 \quad-1.898930$

$\mathrm{H} \quad-8.513599 \quad-1.734209 \quad-1.730803$

$\mathrm{H} \quad-7.832124 \quad-2.022513 \quad-0.120698$

$\begin{array}{llll}\text { C } & -7.179106 & 0.574648 & 0.557681\end{array}$

$\begin{array}{llll}\text { C } & -6.852496 & 2.074415 & 0.525624\end{array}$

$\begin{array}{llll}\text { C } & -8.075052 & 0.239206 & 1.743901\end{array}$

$\mathrm{H} \quad-7.760642 \quad 2.677243 \quad 0.421238$

$\begin{array}{llll}\mathrm{H} & -6.164038 & 2.307177 & -0.292406\end{array}$

$\begin{array}{llll}\mathrm{H} & -9.066877 & 0.687823 & 1.617493\end{array}$

$\begin{array}{llll}\mathrm{H} & -8.190402 & -0.839741 & 1.868644\end{array}$

$\begin{array}{llll}\mathrm{H} & -6.359427 & 2.355514 & 1.460613\end{array}$

$\begin{array}{llll}\mathrm{H} & -7.634750 & 0.640209 & 2.662763\end{array}$

\section{TS-6 E= -3945.85524754}

$\begin{array}{llll}\text { C } & 3.415008 & 2.646247 & -0.352203\end{array}$

$\begin{array}{llll}\text { C } & 2.898300 \quad 3.516185 & 0.631805\end{array}$

$\begin{array}{llll}\text { C } & 2.437365 & 4.778158 & 0.234400\end{array}$

$\begin{array}{llll}\text { C } & 2.444222 & 5.190797 & -1.097733\end{array}$

$\begin{array}{llll}\text { C } & 2.922700 & 4.293892 & -2.056374\end{array}$

$\begin{array}{llll}\text { C } & 3.412325 & 3.031109 & -1.712809\end{array}$

$\begin{array}{llll}\text { O } & 2.092417 & 0.131799 & -0.712720\end{array}$

$\begin{array}{llll}\mathrm{P} & 0.973605 & -0.278067 & 0.405939\end{array}$

$\begin{array}{llll}\text { C } & 3.404258 & 0.098110 & -0.221930\end{array}$

$\begin{array}{llll}\text { O } & -0.344660 & -0.375792 & -0.439138\end{array}$

$\begin{array}{llll}\text { O } & 1.373690 & -1.809162 & 0.765160\end{array}$

$\begin{array}{llll}\text { C } & 4.008977 & -1.135109 & -0.037576\end{array}$

$\begin{array}{llll}\text { C } & 4.043187 & 1.341007 & 0.030809\end{array}$

$\begin{array}{llll}\text { C } & 2.023946 & -2.649296 & -0.133803\end{array}$

$\begin{array}{llll}\text { C } & 3.325310 & -2.369463 & -0.518650\end{array}$

$\begin{array}{llll}\text { C } & 5.313003 & 1.297400 & 0.565713\end{array}$

$\begin{array}{llll}\text { C } & 5.290970 & -1.165685 & 0.609987\end{array}$

$\begin{array}{llll}\text { C } & 5.945620 & 0.073939 & 0.905333\end{array}$

$\begin{array}{llll}\text { C } & 1.309378 & -3.806104 & -0.543160\end{array}$

$\begin{array}{llll}\text { C } & 3.962202 & -3.267916 & -1.444257\end{array}$

$\begin{array}{llll}\text { C } & 1.977193 & -4.720991 & -1.326926\end{array}$

$\begin{array}{llll}\text { C } & 3.288367 & -4.478729 & -1.809514\end{array}$

$\begin{array}{llll}\text { C } & -0.120157 & -4.003806 & -0.143140\end{array}$

$\begin{array}{llll}\text { C } & -1.149527 & -3.566734 & -1.011196\end{array}$

$\begin{array}{llll}\text { C } & -2.476186 & -3.774158 & -0.627683\end{array}$

$\begin{array}{llll}\text { C } & -2.815593 & -4.408816 & 0.572930\end{array}$

$\begin{array}{llll}\text { C } & -1.781197 & -4.817179 & 1.412962\end{array}$

$\begin{array}{llll}\text { C } & -0.433908 & -4.616526 & 1.084146\end{array}$

\section{SI-44}




\begin{tabular}{|c|c|c|c|c|c|c|c|}
\hline $\mathrm{O}$ & 0.965836 & 0.553966 & 1.630809 & $\mathrm{H}$ & -0.386684 & -6.724455 & 3.010921 \\
\hline $\mathrm{H}$ & 2.054733 & 5.460753 & 0.988648 & $\mathrm{H}$ & 0.787885 & -3.076735 & 3.023910 \\
\hline $\mathrm{H}$ & 2.924926 & 4.588647 & -3.102512 & $\mathrm{H}$ & -0.306263 & -4.200328 & 3.851225 \\
\hline $\mathrm{H}$ & 5.842964 & 2.229199 & 0.741771 & $\mathrm{H}$ & 1.449028 & -4.405528 & 4.001914 \\
\hline $\mathrm{H}$ & 1.473175 & -5.640006 & -1.612969 & $\mathrm{C}$ & -0.832258 & -2.922708 & -2.359550 \\
\hline $\mathrm{H}$ & -3.271665 & -3.431829 & -1.280948 & $\mathrm{C}$ & -1.883486 & -1.900141 & -2.813704 \\
\hline $\mathrm{H}$ & -2.029998 & -5.295483 & 2.357679 & $\mathrm{C}$ & -0.664197 & -3.997735 & -3.453449 \\
\hline $\mathrm{H}$ & -1.005943 & 0.407511 & -0.318468 & $\mathrm{H}$ & 0.118989 & -2.389221 & -2.259027 \\
\hline $\mathrm{C}$ & -4.337069 & 0.675172 & 0.907563 & $\mathrm{H}$ & -2.150569 & -1.194841 & -2.026604 \\
\hline $\mathrm{C}$ & -3.395524 & -0.324014 & 1.294756 & $\mathrm{H}$ & -1.502894 & -1.332985 & -3.671292 \\
\hline $\mathrm{C}$ & -2.885170 & -0.502089 & 2.583454 & $\mathrm{H}$ & -2.804113 & -2.397255 & -3.141886 \\
\hline $\mathrm{O}$ & -3.659198 & 3.122351 & 0.000289 & $\mathrm{H}$ & 0.145439 & -4.694496 & -3.223254 \\
\hline B & -3.080898 & 1.988530 & 0.618775 & $\mathrm{H}$ & -1.589201 & -4.577616 & -3.561468 \\
\hline $\mathrm{O}$ & -2.669833 & 2.216878 & 2.016603 & $\mathrm{H}$ & -0.441755 & -3.529085 & -4.419910 \\
\hline $\mathrm{O}$ & -1.957536 & 1.571669 & -0.227069 & $\mathrm{C}$ & -4.272647 & -4.642428 & 0.947053 \\
\hline $\mathrm{C}$ & -1.874243 & 1.343678 & 2.603141 & $\mathrm{C}$ & -5.010701 & -5.463175 & -0.126755 \\
\hline $\mathrm{C}$ & -1.628282 & 1.538597 & 4.044457 & $\mathrm{C}$ & -5.006951 & -3.317124 & 1.220800 \\
\hline $\mathrm{C}$ & -2.546547 & 2.223737 & 4.855226 & $\mathrm{H}$ & -4.283948 & -5.227972 & 1.876764 \\
\hline $\mathrm{C}$ & -0.441180 & 1.041127 & 4.606491 & $\mathrm{H}$ & -4.495011 & -6.408807 & -0.326450 \\
\hline $\mathrm{C}$ & -0.189041 & 1.213707 & 5.967921 & $\mathrm{H}$ & -6.034027 & -5.689244 & 0.195877 \\
\hline $\mathrm{C}$ & -2.288119 & 2.393617 & 6.212197 & $\mathrm{H}$ & -5.075484 & -4.910998 & -1.071750 \\
\hline $\mathrm{C}$ & -1.111611 & 1.884185 & 6.773381 & $\mathrm{H}$ & -4.552489 & -2.774965 & 2.055070 \\
\hline $\mathrm{H}$ & -2.871683 & -0.839365 & 0.489521 & $\mathrm{H}$ & -4.966895 & -2.660945 & 0.344865 \\
\hline $\mathrm{H}$ & -3.528362 & -0.229375 & 3.416653 & $\mathrm{H}$ & -6.061548 & -3.497386 & 1.462835 \\
\hline $\mathrm{H}$ & -1.028156 & 0.983645 & 2.027961 & $\mathrm{C}$ & 2.838998 & 3.131258 & 2.107355 \\
\hline $\mathrm{H}$ & -3.447466 & 2.629160 & 4.405487 & $\mathrm{C}$ & 1.475335 & 3.455268 & 2.742306 \\
\hline $\mathrm{H}$ & 0.286791 & 0.556915 & 3.962825 & $\mathrm{C}$ & 3.976693 & 3.805461 & 2.897439 \\
\hline $\mathrm{H}$ & 0.733469 & 0.831596 & 6.396657 & $\mathrm{H}$ & 2.966831 & 2.047826 & 2.177106 \\
\hline $\mathrm{H}$ & -3.001103 & 2.926729 & 6.835549 & $\mathrm{H}$ & 0.663955 & 3.001211 & 2.169012 \\
\hline $\mathrm{H}$ & -0.913254 & 2.018156 & 7.833520 & $\mathrm{H}$ & 1.427965 & 3.050115 & 3.758501 \\
\hline $\mathrm{C}$ & 0.651735 & -5.035096 & 2.070039 & $\mathrm{H}$ & 1.297092 & 4.534911 & 2.810584 \\
\hline $\mathrm{C}$ & 0.540815 & -6.518371 & 2.464193 & $\mathrm{H}$ & 4.960607 & 3.522555 & 2.507954 \\
\hline $\mathrm{C}$ & 0.645144 & -4.122596 & 3.311388 & $\mathrm{H}$ & 3.896241 & 4.898099 & 2.840555 \\
\hline $\mathrm{H}$ & 1.621503 & -4.906254 & 1.578410 & $\mathrm{H}$ & 3.934603 & 3.517770 & 3.954944 \\
\hline $\mathrm{H}$ & 0.557997 & -7.164635 & 1.579339 & $\mathrm{C}$ & 3.929334 & 2.115884 & -2.820480 \\
\hline $\mathrm{H}$ & 1.376694 & -6.803647 & 3.113941 & $\mathrm{C}$ & 5.041499 & 2.785198 & -3.648359 \\
\hline
\end{tabular}

\section{SI-45}




\begin{tabular}{|c|c|c|c|}
\hline $\mathrm{C}$ & 2.777091 & 1.629393 & -3.719536 \\
\hline $\mathrm{H}$ & 4.373209 & 1.229906 & -2.357193 \\
\hline $\mathrm{H}$ & 5.863511 & 3.119738 & -3.005648 \\
\hline $\mathrm{H}$ & 5.445737 & 2.079534 & -4.383892 \\
\hline $\mathrm{H}$ & 4.669964 & 3.657968 & -4.197666 \\
\hline $\mathrm{H}$ & 2.033536 & 1.081882 & -3.133207 \\
\hline $\mathrm{H}$ & 2.274788 & 2.472977 & -4.208332 \\
\hline $\mathrm{H}$ & 3.156172 & 0.962242 & -4.503300 \\
\hline $\mathrm{C}$ & 1.974577 & 6.585476 & -1.486591 \\
\hline $\mathrm{C}$ & 0.839739 & 6.560485 & -2.526125 \\
\hline $\mathrm{C}$ & 3.154655 & 7.438952 & -1.989292 \\
\hline $\mathrm{H}$ & 1.580530 & 7.063740 & -0.579392 \\
\hline $\mathrm{H}$ & -0.027389 & 6.004343 & -2.155353 \\
\hline $\mathrm{H}$ & 0.512499 & 7.579492 & -2.764213 \\
\hline $\mathrm{H}$ & 1.165316 & 6.089448 & -3.461084 \\
\hline $\mathrm{H}$ & 3.951183 & 7.489203 & -1.238978 \\
\hline $\mathrm{H}$ & 3.583674 & 7.010588 & -2.903146 \\
\hline $\mathrm{H}$ & 2.829039 & 8.461261 & -2.216646 \\
\hline $\mathrm{C}$ & 7.218602 & 0.054314 & 1.535991 \\
\hline $\mathrm{H}$ & 7.708015 & 1.002475 & 1.745344 \\
\hline $\mathrm{C}$ & 7.814190 & -1.134205 & 1.890187 \\
\hline $\mathrm{H}$ & 8.784782 & -1.136224 & 2.378398 \\
\hline $\mathrm{C}$ & 7.152698 & -2.358194 & 1.631567 \\
\hline $\mathrm{C}$ & 5.925330 & -2.374354 & 1.007033 \\
\hline $\mathrm{H}$ & 5.425272 & -3.318029 & 0.821453 \\
\hline $\mathrm{C}$ & 3.925480 & -5.394525 & -2.689407 \\
\hline $\mathrm{C}$ & 5.163095 & -5.118996 & -3.222599 \\
\hline $\mathrm{H}$ & 5.637803 & -5.823471 & -3.899892 \\
\hline $\mathrm{C}$ & 5.809676 & -3.901819 & -2.902879 \\
\hline $\mathrm{C}$ & 5.226585 & -3.001482 & -2.039017 \\
\hline $\mathrm{H}$ & 5.733266 & -2.071112 & -1.812051 \\
\hline $\mathrm{H}$ & 7.614960 & -3.294063 & 1.933267 \\
\hline $\mathrm{H}$ & 3.404046 & -6.313435 & -2.946728 \\
\hline $\mathrm{H}$ & 6.774032 & -3.671595 & -3.347440 \\
\hline $\mathrm{C}$ & -5.324211 & 1.212116 & 1.957643 \\
\hline $\mathrm{C}$ & -2.590825 & 3.787478 & -0.706253 \\
\hline
\end{tabular}

\begin{tabular}{|c|c|c|c|}
\hline $\mathrm{C}$ & -1.819737 & 4.675387 & 0.286696 \\
\hline$C$ & -3.208204 & 4.660741 & -1.795759 \\
\hline$H$ & -1.046053 & 5.264221 & -0.216468 \\
\hline $\mathrm{H}$ & -1.344807 & 4.086043 & 1.073333 \\
\hline $\mathrm{H}$ & -2.429114 & 5.125519 & -2.411114 \\
\hline $\mathrm{H}$ & -3.869693 & 4.084687 & -2.445760 \\
\hline $\mathrm{C}$ & -1.725483 & 2.589134 & -1.247531 \\
\hline $\mathrm{C}$ & -2.225630 & 2.026754 & -2.578170 \\
\hline $\mathrm{C}$ & -0.227151 & 2.857087 & -1.332055 \\
\hline $\mathrm{H}$ & -2.038083 & 2.726398 & -3.399506 \\
\hline $\mathrm{H}$ & -3.288443 & 1.799199 & -2.525008 \\
\hline $\mathrm{H}$ & -0.018630 & 3.680666 & -2.019903 \\
\hline $\mathrm{H}$ & 0.205517 & 3.104057 & -0.362256 \\
\hline $\mathrm{H}$ & -2.528711 & 5.359587 & 0.762637 \\
\hline $\mathrm{H}$ & -3.799894 & 5.456469 & -1.332514 \\
\hline $\mathrm{H}$ & 0.293351 & 1.975301 & -1.714366 \\
\hline $\mathrm{H}$ & -1.699494 & 1.092412 & -2.793543 \\
\hline $\mathrm{H}$ & -5.760630 & 2.151506 & 1.609191 \\
\hline $\mathrm{H}$ & -6.146157 & 0.500700 & 2.105446 \\
\hline $\mathrm{H}$ & -4.855249 & 1.402531 & 2.923789 \\
\hline B & -5.032349 & 0.471226 & -0.479621 \\
\hline $\mathrm{O}$ & -6.337149 & 0.850333 & -0.677507 \\
\hline $\mathrm{O}$ & -4.479000 & -0.141019 & -1.581073 \\
\hline $\mathrm{C}$ & -5.554755 & -0.364948 & -2.540591 \\
\hline $\mathrm{C}$ & -5.019979 & -0.164721 & -3.955361 \\
\hline $\mathrm{C}$ & -6.012247 & -1.816121 & -2.352116 \\
\hline $\mathrm{H}$ & -4.256270 & -0.915555 & -4.174444 \\
\hline $\mathrm{H}$ & -4.568915 & 0.819870 & -4.085585 \\
\hline $\mathrm{H}$ & -5.153787 & -2.479563 & -2.492293 \\
\hline $\mathrm{H}$ & -6.404121 & -1.977858 & -1.343960 \\
\hline $\mathrm{C}$ & -6.635229 & 0.688850 & -2.087959 \\
\hline $\mathrm{C}$ & -6.455323 & 2.068876 & -2.730796 \\
\hline $\mathrm{C}$ & -8.082724 & 0.226529 & -2.229980 \\
\hline $\mathrm{H}$ & -6.695743 & 2.054539 & -3.798739 \\
\hline $\mathrm{H}$ & -5.432633 & 2.430193 & -2.598667 \\
\hline $\mathrm{H}$ & -8.322010 & 0.020778 & -3.279485 \\
\hline
\end{tabular}




$\begin{array}{llll}\mathrm{H} & -8.277259 & -0.673092 & -1.642250 \\ \mathrm{H} & -7.121116 & 2.778085 & -2.230547 \\ \mathrm{H} & -8.754747 & 1.013372 & -1.873824 \\ \mathrm{H} & -6.785103 & -2.092609 & -3.076361 \\ \mathrm{H} & -5.830859 & -0.275858 & -4.684314 \\ \mathrm{C} & -1.877340 & -1.587382 & 2.870345 \\ \mathrm{H} & -1.265080 & -1.814852 & 1.993332 \\ \mathrm{H} & -1.214540 & -1.308193 & 3.695242 \\ \mathrm{H} & -2.379459 & -2.518114 & 3.159866\end{array}$

\section{TS-7 E= -3945.84069847}

\begin{tabular}{lrrr}
$\mathrm{O}$ & -2.475554 & 0.370116 & 0.662229 \\
$\mathrm{P}$ & -1.130951 & -0.297805 & 0.043818 \\
$\mathrm{O}$ & -1.743446 & -1.485451 & -0.876888 \\
$\mathrm{O}$ & -0.214527 & -0.678017 & 1.146528 \\
$\mathrm{O}$ & -0.563626 & 0.645708 & -1.078485 \\
$\mathrm{H}$ & 0.401732 & 0.974606 & -0.940362 \\
$\mathrm{C}$ & -3.664385 & 0.546332 & -0.037970 \\
$\mathrm{C}$ & -4.392759 & -0.558538 & -0.462519 \\
$\mathrm{C}$ & -4.130395 & 1.887724 & -0.164068 \\
$\mathrm{C}$ & -5.590173 & -0.322676 & -1.227925 \\
$\mathrm{C}$ & -5.379878 & 2.069348 & -0.722461 \\
$\mathrm{C}$ & -6.111902 & 1.006785 & -1.303118 \\
$\mathrm{H}$ & -5.786375 & 3.074741 & -0.781504 \\
$\mathrm{C}$ & -2.671988 & -2.374608 & -0.340382 \\
$\mathrm{C}$ & -3.971804 & -1.946637 & -0.125218 \\
$\mathrm{C}$ & -2.229620 & -3.708887 & -0.123603 \\
$\mathrm{C}$ & -4.899631 & -2.886740 & 0.454054 \\
$\mathrm{C}$ & -3.179371 & -4.634223 & 0.253939 \\
$\mathrm{C}$ & -4.506472 & -4.258309 & 0.577598 \\
$\mathrm{H}$ & -2.879795 & -5.669899 & 0.391008 \\
$\mathrm{C}$ & -0.780249 & -4.065403 & -0.184476 \\
$\mathrm{C}$ & -0.128033 & -4.464117 & 1.008123 \\
\hline & -0.060867 & -4.007248 & -1.400508 \\
$\mathrm{C}$ & 1.230215 & -4.797368 & 0.945561 \\
$\mathrm{C}$ & 1.298656 & -4.331945 & -1.398916
\end{tabular}

\begin{tabular}{|c|c|c|c|}
\hline $\mathrm{C}$ & 1.965987 & -4.735898 & -0.238251 \\
\hline $\mathrm{H}$ & 1.733176 & -5.115760 & 1.855512 \\
\hline $\mathrm{H}$ & 1.844313 & -4.299381 & -2.338142 \\
\hline$C$ & -3.310043 & 3.076296 & 0.227383 \\
\hline$C$ & -2.991671 & 4.047598 & -0.761220 \\
\hline C & -2.902275 & 3.282994 & 1.566408 \\
\hline$C$ & -2.288558 & 5.193898 & -0.378134 \\
\hline $\mathrm{C}$ & -2.183981 & 4.441343 & 1.884032 \\
\hline $\mathrm{C}$ & -1.874864 & 5.416303 & 0.936672 \\
\hline $\mathrm{H}$ & -2.058711 & 5.939662 & -1.134555 \\
\hline $\mathrm{H}$ & -1.882933 & 4.605447 & 2.916033 \\
\hline $\mathrm{C}$ & -0.844106 & -4.577829 & 2.356745 \\
\hline $\mathrm{C}$ & -0.260915 & -3.644846 & 3.431455 \\
\hline $\mathrm{C}$ & -0.867302 & -6.039260 & 2.843081 \\
\hline $\mathrm{H}$ & -1.882033 & -4.269516 & 2.223892 \\
\hline $\mathrm{H}$ & -0.318631 & -2.601333 & 3.107486 \\
\hline $\mathrm{H}$ & -0.831856 & -3.748962 & 4.362343 \\
\hline $\mathrm{H}$ & 0.785370 & -3.879845 & 3.656234 \\
\hline $\mathrm{H}$ & -1.324896 & -6.700039 & 2.097526 \\
\hline $\mathrm{H}$ & 0.144856 & -6.412931 & 3.037922 \\
\hline $\mathrm{H}$ & -1.440319 & -6.124896 & 3.774168 \\
\hline $\mathrm{C}$ & -0.744209 & -3.703180 & -2.731641 \\
\hline $\mathrm{C}$ & -0.753502 & -4.959412 & -3.625308 \\
\hline $\mathrm{C}$ & -0.117258 & -2.505691 & -3.464384 \\
\hline $\mathrm{H}$ & -1.789012 & -3.449343 & -2.531709 \\
\hline $\mathrm{H}$ & -1.231872 & -5.802947 & -3.114735 \\
\hline $\mathrm{H}$ & -1.300719 & -4.765268 & -4.555674 \\
\hline $\mathrm{H}$ & 0.265048 & -5.264859 & -3.892571 \\
\hline $\mathrm{H}$ & -0.178342 & -1.601496 & -2.854368 \\
\hline $\mathrm{H}$ & 0.935699 & -2.685782 & -3.708629 \\
\hline $\mathrm{H}$ & -0.648478 & -2.318122 & -4.405306 \\
\hline $\mathrm{C}$ & 3.420576 & -5.186326 & -0.273553 \\
\hline $\mathrm{C}$ & 4.371548 & -4.138849 & -0.878490 \\
\hline C & 3.544083 & -6.529275 & -1.020350 \\
\hline $\mathrm{H}$ & 3.734821 & -5.354971 & 0.765944 \\
\hline $\mathrm{H}$ & 4.346954 & -3.204082 & -0.311803 \\
\hline
\end{tabular}




\begin{tabular}{|c|c|c|c|}
\hline $\mathrm{H}$ & 5.402679 & -4.512715 & -0.876395 \\
\hline $\mathrm{H}$ & 4.108484 & -3.907719 & -1.917065 \\
\hline $\mathrm{H}$ & 2.896412 & -7.290910 & -0.572476 \\
\hline $\mathrm{H}$ & 3.250141 & -6.416659 & -2.070876 \\
\hline $\mathrm{H}$ & 4.577457 & -6.895886 & -0.996315 \\
\hline $\mathrm{C}$ & -3.294151 & 2.350836 & 2.711781 \\
\hline $\mathrm{C}$ & -4.255438 & 3.063649 & 3.683922 \\
\hline $\mathrm{C}$ & -2.067381 & 1.791180 & 3.455950 \\
\hline $\mathrm{H}$ & -3.841048 & 1.499624 & 2.301166 \\
\hline $\mathrm{H}$ & -5.142084 & 3.435650 & 3.158035 \\
\hline $\mathrm{H}$ & -4.586643 & 2.372947 & 4.468819 \\
\hline $\mathrm{H}$ & -3.773071 & 3.918571 & 4.171926 \\
\hline $\mathrm{H}$ & -1.413131 & 1.239754 & 2.776208 \\
\hline $\mathrm{H}$ & -1.482834 & 2.590235 & 3.927451 \\
\hline $\mathrm{H}$ & -2.388498 & 1.104372 & 4.248537 \\
\hline $\mathrm{C}$ & -3.405292 & 3.930085 & -2.232425 \\
\hline $\mathrm{C}$ & -2.205830 & 3.938667 & -3.195888 \\
\hline $\mathrm{C}$ & -4.411987 & 5.034991 & -2.608356 \\
\hline $\mathrm{H}$ & -3.905525 & 2.971614 & -2.380722 \\
\hline $\mathrm{H}$ & -1.556348 & 3.078963 & -3.015295 \\
\hline $\mathrm{H}$ & -2.557907 & 3.883749 & -4.233003 \\
\hline $\mathrm{H}$ & -1.600460 & 4.846668 & -3.096410 \\
\hline $\mathrm{H}$ & -5.284927 & 5.026831 & -1.945933 \\
\hline $\mathrm{H}$ & -3.956471 & 6.029667 & -2.536674 \\
\hline $\mathrm{H}$ & -4.763070 & 4.900819 & -3.638487 \\
\hline $\mathrm{C}$ & -1.175949 & 6.706249 & 1.342720 \\
\hline $\mathrm{C}$ & -2.118715 & 7.912817 & 1.169537 \\
\hline $\mathrm{C}$ & 0.145679 & 6.934370 & 0.588571 \\
\hline $\mathrm{H}$ & -0.935465 & 6.625008 & 2.411652 \\
\hline $\mathrm{H}$ & -3.048715 & 7.770069 & 1.730554 \\
\hline $\mathrm{H}$ & -1.640346 & 8.834552 & 1.521971 \\
\hline $\mathrm{H}$ & -2.383504 & 8.053803 & 0.114717 \\
\hline $\mathrm{H}$ & 0.850953 & 6.116565 & 0.763558 \\
\hline $\mathrm{H}$ & -0.020858 & 7.005494 & -0.492774 \\
\hline $\mathrm{H}$ & 0.617112 & 7.868992 & 0.915047 \\
\hline $\mathrm{C}$ & -5.429541 & -5.208763 & 1.091822 \\
\hline
\end{tabular}

\begin{tabular}{|c|c|c|c|}
\hline C & -6.679790 & -4.822304 & 1.514854 \\
\hline $\mathrm{H}$ & -7.374811 & -5.555253 & 1.915012 \\
\hline $\mathrm{C}$ & -7.049600 & -3.458317 & 1.452526 \\
\hline $\mathrm{H}$ & -8.022987 & -3.146796 & 1.821310 \\
\hline$C$ & -6.185763 & -2.517937 & 0.935865 \\
\hline $\mathrm{H}$ & -6.485113 & -1.477428 & 0.904783 \\
\hline$C$ & -6.278216 & -1.349788 & -1.930853 \\
\hline $\mathrm{H}$ & -5.873525 & -2.354882 & -1.929184 \\
\hline $\mathrm{C}$ & -7.438889 & -1.082983 & -2.622689 \\
\hline $\mathrm{H}$ & -7.939017 & -1.883681 & -3.160520 \\
\hline$C$ & -7.982377 & 0.223421 & -2.645944 \\
\hline C & -7.325951 & 1.246279 & -2.002403 \\
\hline $\mathrm{H}$ & -5.116127 & -6.247423 & 1.165144 \\
\hline $\mathrm{H}$ & -8.904250 & 0.417546 & -3.187096 \\
\hline $\mathrm{H}$ & -7.712563 & 2.262057 & -2.036895 \\
\hline $\mathrm{O}$ & 3.983964 & 2.190576 & -1.416036 \\
\hline B & 3.172624 & 1.320515 & -0.633995 \\
\hline $\mathrm{O}$ & 3.423575 & 1.494224 & 0.822057 \\
\hline $\mathrm{O}$ & 1.776525 & 1.653439 & -0.945866 \\
\hline $\mathrm{C}$ & 2.642483 & 0.860197 & 1.659549 \\
\hline $\mathrm{C}$ & 2.893916 & 1.039555 & 3.095657 \\
\hline $\mathrm{C}$ & 4.150904 & 1.449531 & 3.566587 \\
\hline $\mathrm{C}$ & 1.848168 & 0.803217 & 4.001355 \\
\hline $\mathrm{C}$ & 2.057389 & 0.985131 & 5.368031 \\
\hline $\mathrm{C}$ & 4.353533 & 1.626788 & 4.931668 \\
\hline $\mathrm{C}$ & 3.308141 & 1.396618 & 5.834328 \\
\hline $\mathrm{C}$ & 3.536313 & -0.372632 & -0.984664 \\
\hline $\mathrm{C}$ & 2.899842 & -1.109522 & 0.082647 \\
\hline $\mathrm{C}$ & 3.306047 & -1.149473 & 1.410116 \\
\hline $\mathrm{H}$ & 1.602776 & 0.703336 & 1.376009 \\
\hline $\mathrm{H}$ & 4.947025 & 1.621162 & 2.848475 \\
\hline $\mathrm{H}$ & 0.881955 & 0.476937 & 3.624786 \\
\hline $\mathrm{H}$ & 1.245001 & 0.806534 & 6.067004 \\
\hline $\mathrm{H}$ & 5.325778 & 1.945763 & 5.297795 \\
\hline $\mathrm{H}$ & 3.470905 & 1.538135 & 6.899512 \\
\hline $\mathrm{H}$ & 1.931561 & -1.563530 & -0.135542 \\
\hline
\end{tabular}




\begin{tabular}{|c|c|c|c|}
\hline $\mathrm{H}$ & 4.329570 & -0.861642 & 1.631189 \\
\hline $\mathrm{C}$ & 2.959037 & -0.678192 & -2.376956 \\
\hline $\mathrm{C}$ & 1.747399 & 2.863863 & -1.754035 \\
\hline $\mathrm{C}$ & 1.350429 & 2.454981 & -3.176710 \\
\hline $\mathrm{C}$ & 0.697700 & 3.784585 & -1.143158 \\
\hline $\mathrm{H}$ & 0.407946 & 1.901311 & -3.144652 \\
\hline $\mathrm{H}$ & 2.106172 & 1.804118 & -3.620925 \\
\hline $\mathrm{H}$ & -0.287917 & 3.321563 & -1.198769 \\
\hline $\mathrm{H}$ & 0.904664 & 3.974199 & -0.089225 \\
\hline $\mathrm{C}$ & 3.232757 & 3.390238 & -1.648386 \\
\hline $\mathrm{C}$ & 3.761086 & 4.039342 & -2.927385 \\
\hline $\mathrm{C}$ & 3.454380 & 4.347310 & -0.463622 \\
\hline $\mathrm{H}$ & 3.158678 & 4.911701 & -3.205785 \\
\hline $\mathrm{H}$ & 3.765939 & 3.331698 & -3.758817 \\
\hline $\mathrm{H}$ & 2.950771 & 5.307630 & -0.619410 \\
\hline $\mathrm{H}$ & 3.104743 & 3.912025 & 0.474885 \\
\hline $\mathrm{H}$ & 0.651443 & 4.743331 & -1.669770 \\
\hline $\mathrm{H}$ & 1.212990 & 3.330074 & -3.820063 \\
\hline $\mathrm{H}$ & 4.529018 & 4.529929 & -0.364055 \\
\hline $\mathrm{H}$ & 4.791083 & 4.373391 & -2.764888 \\
\hline $\mathrm{H}$ & 3.179445 & -1.709458 & -2.676604 \\
\hline $\mathrm{H}$ & 1.874657 & -0.542574 & -2.398000 \\
\hline $\mathrm{H}$ & 3.404527 & -0.016160 & -3.122801 \\
\hline $\mathrm{C}$ & 2.623765 & -2.047890 & 2.400241 \\
\hline $\mathrm{H}$ & 2.695824 & -1.655289 & 3.419503 \\
\hline $\mathrm{H}$ & 1.573331 & -2.169937 & 2.141411 \\
\hline $\mathrm{H}$ & 3.093859 & -3.039823 & 2.396835 \\
\hline B & 5.101159 & -0.308888 & -0.979313 \\
\hline $\mathrm{O}$ & 5.885003 & -0.226653 & 0.151766 \\
\hline $\mathrm{O}$ & 5.834492 & -0.293423 & -2.138722 \\
\hline $\mathrm{C}$ & 7.238205 & -0.331346 & -1.769233 \\
\hline $\mathrm{C}$ & 8.016045 & 0.546325 & -2.744317 \\
\hline $\mathrm{C}$ & 7.681267 & -1.794795 & -1.885251 \\
\hline $\mathrm{H}$ & 7.972452 & 0.110639 & -3.747609 \\
\hline $\mathrm{H}$ & 7.591358 & 1.551079 & -2.793292 \\
\hline $\mathrm{H}$ & 7.475438 & -2.145923 & -2.900925 \\
\hline
\end{tabular}

$\begin{array}{lrrr}\mathrm{H} & 7.120769 & -2.427139 & -1.189215 \\ \mathrm{C} & 7.207854 & 0.191183 & -0.285679 \\ \mathrm{C} & 7.231128 & 1.721636 & -0.182167 \\ \mathrm{C} & 8.246066 & -0.429950 & 0.641946 \\ \mathrm{H} & 8.206875 & 2.130251 & -0.465537 \\ \mathrm{H} & 6.450323 & 2.163154 & -0.807875 \\ \mathrm{H} & 9.260195 & -0.197683 & 0.297379 \\ \mathrm{H} & 8.135160 & -1.515034 & 0.700741 \\ \mathrm{H} & 7.023707 & 2.004636 & 0.854886 \\ \mathrm{H} & 8.126996 & -0.022604 & 1.651093 \\ \mathrm{H} & 8.751462 & -1.912355 & -1.685158 \\ \mathrm{H} & 9.068593 & 0.619084 & -2.447124\end{array}$




\begin{tabular}{|c|c|c|c|}
\hline $\mathrm{H}$ & -2.314914 & -0.130478 & 1.340249 \\
\hline $\mathrm{H}$ & -3.469633 & -0.525874 & -2.227526 \\
\hline $\mathrm{H}$ & -4.586049 & -0.712236 & 1.916400 \\
\hline $\mathrm{H}$ & -6.936216 & -1.181912 & 1.270634 \\
\hline $\mathrm{H}$ & -5.824314 & -0.995756 & -2.883524 \\
\hline $\mathrm{H}$ & -7.556721 & -1.330901 & -1.132273 \\
\hline $\mathrm{C}$ & -0.100427 & -1.665622 & -2.086971 \\
\hline $\mathrm{C}$ & 0.284268 & 2.548651 & -1.414475 \\
\hline $\mathrm{C}$ & -1.114441 & 3.160084 & -1.611501 \\
\hline $\mathrm{C}$ & 1.268728 & 3.171124 & -2.402981 \\
\hline $\mathrm{H}$ & -1.090434 & 4.252688 & -1.552264 \\
\hline $\mathrm{H}$ & -1.824877 & 2.783662 & -0.872063 \\
\hline $\mathrm{H}$ & 1.451173 & 4.225705 & -2.168656 \\
\hline $\mathrm{H}$ & 2.221100 & 2.636184 & -2.400231 \\
\hline $\mathrm{C}$ & 0.770693 & 2.605395 & 0.070177 \\
\hline $\mathrm{C}$ & 2.274098 & 2.417994 & 0.237522 \\
\hline $\mathrm{C}$ & 0.302065 & 3.863601 & 0.799562 \\
\hline $\mathrm{H}$ & 2.812125 & 3.318868 & -0.074858 \\
\hline $\mathrm{H}$ & 2.621735 & 1.569052 & -0.348435 \\
\hline $\mathrm{H}$ & 0.627039 & 4.730789 & 0.213544 \\
\hline $\mathrm{H}$ & -0.780718 & 3.894380 & 0.909085 \\
\hline $\mathrm{H}$ & -1.474196 & 2.871718 & -2.603615 \\
\hline $\mathrm{H}$ & 0.851137 & 3.111420 & -3.412784 \\
\hline $\mathrm{H}$ & 0.746940 & 3.927537 & 1.791920 \\
\hline $\mathrm{H}$ & 2.484175 & 2.217905 & 1.288901 \\
\hline $\mathrm{H}$ & 0.439472 & -1.075986 & -2.829374 \\
\hline $\mathrm{H}$ & 0.166595 & -2.719288 & -2.229465 \\
\hline $\mathrm{H}$ & -1.168367 & -1.548858 & -2.287337 \\
\hline B & 1.844125 & -1.194388 & -0.440983 \\
\hline $\mathrm{O}$ & 2.721291 & -1.692497 & -1.369839 \\
\hline $\mathrm{O}$ & 2.450270 & -0.795539 & 0.721687 \\
\hline $\mathrm{C}$ & 3.821951 & -1.279548 & 0.687325 \\
\hline $\mathrm{C}$ & 4.723540 & -0.236877 & 1.340825 \\
\hline $\mathrm{C}$ & 3.833999 & -2.575788 & 1.506667 \\
\hline $\mathrm{H}$ & 4.436006 & -0.113519 & 2.389338 \\
\hline $\mathrm{H}$ & 4.637229 & 0.735703 & 0.853871 \\
\hline
\end{tabular}

\begin{tabular}{lrrr}
$\mathrm{H}$ & 3.470478 & -2.356372 & 2.514886 \\
$\mathrm{H}$ & 3.170644 & -3.326070 & 1.064543 \\
$\mathrm{C}$ & 4.070627 & -1.509124 & -0.857101 \\
$\mathrm{C}$ & 4.660045 & -0.294833 & -1.582926 \\
$\mathrm{C}$ & 4.883163 & -2.756875 & -1.196566 \\
$\mathrm{H}$ & 5.699399 & -0.117198 & -1.288277 \\
$\mathrm{H}$ & 4.082002 & 0.610607 & -1.383942 \\
$\mathrm{H}$ & 5.888898 & -2.692715 & -0.766296 \\
$\mathrm{H}$ & 4.400806 & -3.664036 & -0.826995 \\
$\mathrm{H}$ & 4.629292 & -0.480276 & -2.660727 \\
$\mathrm{H}$ & 4.980738 & -2.845995 & -2.282955 \\
$\mathrm{H}$ & 4.840458 & -2.999695 & 1.581888 \\
$\mathrm{H}$ & 5.771019 & -0.556963 & 1.305038 \\
$\mathrm{C}$ & -2.316421 & -2.956531 & 1.615614 \\
$\mathrm{H}$ & -1.966116 & -2.470852 & 2.533057 \\
$\mathrm{H}$ & -3.409270 & -2.901041 & 1.585639 \\
$\mathrm{H}$ & -2.047601 & -4.019263 & 1.667023 \\
$\mathrm{~F}$ & -0.305432 & 1.325647 & 2.146539 \\
$\mathrm{~F}$ & -1.503097 & 2.013297 & 2.207507 \\
\hline & 0.711743 & 1.904939 & 2.864089 \\
$\mathrm{~F}$ & -0.485069 & -0.008749 & 2.477989
\end{tabular}

\section{TS-8b E = -1688.15065153}

$\begin{array}{lrrr}\text { C } & 0.175848 & -1.235365 & -0.382268 \\ \text { C } & -0.512368 & -1.981457 & 0.627325 \\ \text { C } & -1.833987 & -2.412971 & 0.520850 \\ \text { O } & 0.294743 & 1.561415 & -0.332406 \\ \text { B } & -0.352682 & 0.380752 & 0.293909 \\ \text { O } & -1.784134 & 0.334240 & -0.040150 \\ \text { O } & -0.107975 & 0.500582 & 1.684428 \\ \text { C } & -2.578077 & -0.430217 & 0.672497 \\ \text { C } & -3.950019 & -0.571454 & 0.149898 \\ \text { C } & -4.217632 & -0.289506 & -1.200935 \\ \text { C } & -4.996202 & -0.963021 & 0.997952 \\ \text { C } & -6.291883 & -1.096237 & 0.498741 \\ \text { C } & -5.512501 & -0.422693 & -1.692310\end{array}$




\begin{tabular}{|c|c|c|c|c|c|c|c|}
\hline $\mathrm{C}$ & -6.550437 & -0.833043 & -0.847729 & $\mathrm{C}$ & 3.976227 & -3.079490 & 0.829553 \\
\hline $\mathrm{H}$ & -0.053551 & -2.009509 & 1.615532 & $\mathrm{H}$ & 4.595209 & -1.109112 & 2.558888 \\
\hline $\mathrm{H}$ & -2.227712 & -2.573611 & -0.479579 & $\mathrm{H}$ & 4.609920 & 0.275858 & 1.458911 \\
\hline $\mathrm{H}$ & -2.446087 & -0.400779 & 1.756448 & $\mathrm{H}$ & 3.696107 & -3.307350 & 1.862607 \\
\hline $\mathrm{H}$ & -3.407574 & 0.056333 & -1.836059 & $\mathrm{H}$ & 3.314866 & -3.643251 & 0.164208 \\
\hline $\mathrm{H}$ & -4.796451 & -1.145136 & 2.050782 & $\mathrm{C}$ & 3.910527 & -1.168185 & -0.920500 \\
\hline $\mathrm{H}$ & -7.098456 & -1.396934 & 1.161563 & $\mathrm{C}$ & 4.207729 & 0.319231 & -1.145000 \\
\hline $\mathrm{H}$ & -5.717157 & -0.200500 & -2.735873 & $\mathrm{C}$ & 4.837949 & -2.030926 & -1.769537 \\
\hline $\mathrm{H}$ & -7.559533 & -0.936210 & -1.237246 & $\mathrm{H}$ & 5.251510 & 0.559086 & -0.916675 \\
\hline $\mathrm{C}$ & -0.289665 & -1.468062 & -1.832708 & $\mathrm{H}$ & 3.553020 & 0.947631 & -0.535332 \\
\hline $\mathrm{C}$ & 0.316723 & 2.633451 & 0.708432 & $\mathrm{H}$ & 5.870213 & -1.958777 & -1.408371 \\
\hline $\mathrm{C}$ & -1.016521 & 3.381275 & 0.622533 & $\mathrm{H}$ & 4.533629 & -3.079886 & -1.757878 \\
\hline $\mathrm{C}$ & 1.487793 & 3.582490 & 0.468521 & $\mathrm{H}$ & 3.997933 & 0.565728 & -2.188162 \\
\hline $\mathrm{H}$ & -1.043552 & 4.194060 & 1.354907 & $\mathrm{H}$ & 4.812743 & -1.682938 & -2.806637 \\
\hline $\mathrm{H}$ & -1.863743 & 2.715574 & 0.800468 & $\mathrm{H}$ & 5.005334 & -3.414846 & 0.665489 \\
\hline $\mathrm{H}$ & 1.636934 & 4.192529 & 1.366237 & $\mathrm{H}$ & 5.809678 & -1.017470 & 1.267885 \\
\hline $\mathrm{H}$ & 2.408762 & 3.032575 & 0.265332 & $\mathrm{C}$ & -2.486395 & -3.226907 & 1.610586 \\
\hline $\mathrm{C}$ & 0.482825 & 1.765077 & 2.011932 & $\mathrm{H}$ & -2.137997 & -2.922041 & 2.604427 \\
\hline $\mathrm{C}$ & 1.946504 & 1.507254 & 2.387495 & $\mathrm{H}$ & -3.576298 & -3.130498 & 1.578140 \\
\hline $\mathrm{C}$ & -0.258893 & 2.341555 & 3.220432 & $\mathrm{H}$ & -2.255154 & -4.292663 & 1.487721 \\
\hline $\mathrm{H}$ & 2.435469 & 2.406984 & 2.774426 & $\mathrm{~B}$ & 0.300443 & 1.857740 & -1.954029 \\
\hline $\mathrm{H}$ & 2.503845 & 1.127963 & 1.533455 & $\mathrm{~F}$ & 1.432899 & 1.213795 & -2.387567 \\
\hline $\mathrm{H}$ & 0.110276 & 3.342234 & 3.472281 & $\mathrm{~F}$ & -0.866864 & 1.318300 & -2.429515 \\
\hline $\mathrm{H}$ & -1.334702 & 2.398018 & 3.040519 & $\mathrm{~F}$ & 0.351793 & 3.222531 & -2.096086 \\
\hline
\end{tabular}

$\begin{array}{lrrr}\mathrm{H} & -1.115755 & 3.807634 & -0.376570 \\ \mathrm{H} & 1.290075 & 4.240522 & -0.374903 \\ \mathrm{H} & -0.093045 & 1.689797 & 4.083993 \\ \mathrm{H} & 1.971718 & 0.731518 & 3.157441 \\ \mathrm{H} & 0.328778 & -0.901169 & -2.525002 \\ \mathrm{H} & -0.187805 & -2.532688 & -2.079734 \\ \mathrm{H} & -1.324940 & -1.170220 & -1.997225 \\ \mathrm{~B} & 1.742483 & -1.260256 & -0.255118 \\ \mathrm{O} & 2.542615 & -1.386824 & -1.358004 \\ \mathrm{O} & 2.446853 & -1.237861 & 0.926299 \\ \mathrm{C} & 3.829297 & -1.571038 & 0.596644 \\ \mathrm{C} & 4.765660 & -0.802045 & 1.522090\end{array}$

\section{TS-8c E $=\mathbf{- 1 6 8 8 . 1 2 8 8 3 7 8 1}$}

$\begin{array}{lrrr}\text { C } & 0.731326 & -0.928388 & -0.756895 \\ \text { C } & -0.107569 & -1.693098 & 0.109200 \\ \text { C } & -1.476809 & -2.041408 & -0.092087 \\ \text { O } & 0.910947 & 1.673550 & -0.829660 \\ \text { B } & 0.456481 & 0.605372 & -0.124454 \\ \text { O } & -1.741450 & 0.456556 & -0.508622 \\ \text { O } & 0.315596 & 0.821501 & 1.225154 \\ \text { C } & -2.175736 & -0.490084 & 0.365479 \\ \text { C } & -3.655352 & -0.733812 & 0.574832 \\ \text { C } & -4.556237 & -1.106399 & -0.434028\end{array}$




\begin{tabular}{|c|c|c|c|c|c|c|c|}
\hline $\mathrm{C}$ & -4.123440 & -0.615529 & 1.891287 & $\mathrm{O}$ & 2.742172 & -1.089721 & 0.914188 \\
\hline $\mathrm{C}$ & -5.465818 & -0.838937 & 2.198993 & $\mathrm{C}$ & 4.162340 & -1.424733 & 0.824203 \\
\hline $\mathrm{C}$ & -5.895163 & -1.333157 & -0.123526 & $\mathrm{C}$ & 4.906529 & -0.697818 & 1.938229 \\
\hline $\mathrm{C}$ & -6.356555 & -1.201017 & 1.189050 & $\mathrm{C}$ & 4.259581 & -2.941853 & 1.017375 \\
\hline $\mathrm{H}$ & 0.273823 & -1.848584 & 1.121103 & $\mathrm{H}$ & 4.562972 & -1.064108 & 2.910527 \\
\hline $\mathrm{H}$ & -1.791800 & -2.102657 & -1.132498 & $\mathrm{H}$ & 4.735101 & 0.379349 & 1.904419 \\
\hline $\mathrm{H}$ & -1.731408 & -0.280104 & 1.340771 & $\mathrm{H}$ & 3.802014 & -3.207061 & 1.975187 \\
\hline $\mathrm{H}$ & -4.212177 & -1.202004 & -1.454469 & $\mathrm{H}$ & 3.724733 & -3.474681 & 0.224755 \\
\hline $\mathrm{H}$ & -3.432227 & -0.333640 & 2.682257 & $\mathrm{C}$ & 4.522872 & -0.957762 & -0.636729 \\
\hline $\mathrm{H}$ & -5.810765 & -0.730516 & 3.223776 & $\mathrm{C}$ & 4.911106 & 0.521549 & -0.730508 \\
\hline $\mathrm{H}$ & -6.584443 & -1.612523 & -0.915689 & $\mathrm{C}$ & 5.550372 & -1.825151 & -1.355370 \\
\hline $\mathrm{H}$ & -7.403155 & -1.379671 & 1.421120 & $\mathrm{H}$ & 5.886078 & 0.712181 & -0.271080 \\
\hline $\mathrm{C}$ & 0.514258 & -0.965677 & -2.280112 & $\mathrm{H}$ & 4.161729 & 1.156625 & -0.249481 \\
\hline $\mathrm{C}$ & 0.718163 & 2.844387 & 0.027516 & $\mathrm{H}$ & 6.505914 & -1.812309 & -0.819208 \\
\hline $\mathrm{C}$ & -0.606012 & 3.499359 & -0.374452 & $\mathrm{H}$ & 5.211969 & -2.859413 & -1.445479 \\
\hline $\mathrm{C}$ & 1.878872 & 3.798256 & -0.240269 & $\mathrm{H}$ & 4.958436 & 0.807221 & -1.785091 \\
\hline $\mathrm{H}$ & -0.746044 & 4.444063 & 0.162215 & $\mathrm{H}$ & 5.718407 & -1.435724 & -2.363891 \\
\hline $\mathrm{H}$ & -1.455853 & 2.844876 & -0.181373 & $\mathrm{H}$ & 5.300351 & -3.280327 & 1.023671 \\
\hline $\mathrm{H}$ & 1.828610 & 4.664747 & 0.428386 & $\mathrm{H}$ & 5.983583 & -0.884206 & 1.862510 \\
\hline $\mathrm{H}$ & 2.846908 & 3.309098 & -0.108490 & $\mathrm{C}$ & -2.010431 & -3.161632 & 0.796020 \\
\hline $\mathrm{C}$ & 0.702693 & 2.206248 & 1.478206 & $\mathrm{H}$ & -1.835269 & -2.944915 & 1.856651 \\
\hline $\mathrm{C}$ & 2.081090 & 2.143159 & 2.141389 & $\mathrm{H}$ & -3.085130 & -3.287131 & 0.651486 \\
\hline $\mathrm{C}$ & -0.319162 & 2.817069 & 2.432153 & $\mathrm{H}$ & -1.518304 & -4.110530 & 0.555846 \\
\hline $\mathrm{H}$ & 2.446644 & 3.139892 & 2.408005 & B & -2.387806 & 0.763473 & -1.875067 \\
\hline $\mathrm{H}$ & 2.805475 & 1.661109 & 1.482074 & $\mathrm{~F}$ & -1.460868 & 1.531649 & -2.543242 \\
\hline $\mathrm{H}$ & -0.102927 & 3.877170 & 2.604394 & $\mathrm{~F}$ & -3.582052 & 1.410526 & -1.648988 \\
\hline $\mathrm{H}$ & -1.332995 & 2.725969 & 2.038193 & $\mathrm{~F}$ & -2.592310 & -0.471654 & -2.541673 \\
\hline
\end{tabular}

$\mathrm{H} \quad-0.590387 \quad 3.692021 \quad-1.448806$

$\mathrm{H} \quad 1.818312 \quad 4.157525 \quad-1.271736$

TS-9a E $=\mathbf{- 1 6 8 8 . 1 6 2 3 4 9 8 1}$

$\begin{array}{lrrr}\mathrm{H} & -0.276786 & 2.298534 & 3.395594 \\ \mathrm{H} & 2.010831 & 1.538101 & 3.050139 \\ \mathrm{H} & 0.992390 & -0.097888 & -2.736828 \\ \mathrm{H} & 0.987056 & -1.865665 & -2.688999 \\ \mathrm{H} & -0.533904 & -0.949785 & -2.562892 \\ \mathrm{~B} & 2.270483 & -1.031112 & -0.370177 \\ \mathrm{O} & 3.242489 & -1.088112 & -1.325207\end{array}$

$\begin{array}{lrrr}\text { C } & -0.181667 & -0.712836 & 1.570727 \\ \text { C } & -0.011718 & 0.536432 & 2.283266 \\ \text { C } & -0.533115 & 1.772007 & 1.931444 \\ \text { O } & 0.266080 & -1.575735 & -0.819460 \\ \text { B } & 0.733394 & -0.590421 & 0.087576 \\ \text { O } & 0.506752 & 0.770773 & -0.423588 \\ \text { O } & 2.221853 & -0.902548 & 0.196601\end{array}$




\begin{tabular}{|c|c|c|c|c|c|c|c|}
\hline $\mathrm{C}$ & 0.718880 & 1.897446 & 0.206492 & $\mathrm{H}$ & -0.230438 & -2.078679 & 3.279274 \\
\hline $\mathrm{C}$ & 0.145568 & 3.097141 & -0.426519 & $\mathrm{~B}$ & -1.604146 & -0.963575 & 0.940441 \\
\hline $\mathrm{C}$ & -0.997630 & 2.995946 & -1.237235 & $\mathrm{O}$ & -2.351781 & -0.002719 & 0.293459 \\
\hline $\mathrm{C}$ & 0.745293 & 4.347228 & -0.221013 & $\mathrm{O}$ & -2.216854 & -2.187608 & 0.970933 \\
\hline $\mathrm{C}$ & 0.212189 & 5.486834 & -0.824822 & $\mathrm{C}$ & -3.549373 & -2.030983 & 0.411310 \\
\hline $\mathrm{C}$ & -1.526656 & 4.134849 & -1.833716 & $\mathrm{C}$ & -3.868518 & -3.271946 & -0.416452 \\
\hline $\mathrm{C}$ & -0.923105 & 5.382201 & -1.630088 & $\mathrm{C}$ & -4.510573 & -1.914679 & 1.599560 \\
\hline $\mathrm{H}$ & 0.716113 & 0.529303 & 3.092403 & $\mathrm{H}$ & -3.926120 & -4.146123 & 0.239638 \\
\hline $\mathrm{H}$ & -1.416624 & 1.784646 & 1.300791 & $\mathrm{H}$ & -3.092724 & -3.459257 & -1.161648 \\
\hline $\mathrm{H}$ & 1.652779 & 2.017425 & 0.750243 & $\mathrm{H}$ & -4.395928 & -2.798273 & 2.234464 \\
\hline $\mathrm{H}$ & -1.456547 & 2.021183 & -1.367798 & $\mathrm{H}$ & -4.279222 & -1.031669 & 2.203614 \\
\hline $\mathrm{H}$ & 1.635144 & 4.419897 & 0.399062 & $\mathrm{C}$ & -3.417498 & -0.696411 & -0.416386 \\
\hline $\mathrm{H}$ & 0.684763 & 6.452527 & -0.669359 & $\mathrm{C}$ & -2.915267 & -0.917632 & -1.848383 \\
\hline $\mathrm{H}$ & -2.411862 & 4.056481 & -2.459155 & $\mathrm{C}$ & -4.655541 & 0.194114 & -0.414789 \\
\hline $\mathrm{H}$ & -1.338975 & 6.269476 & -2.099753 & $\mathrm{H}$ & -3.668337 & -1.419891 & -2.464330 \\
\hline $\mathrm{C}$ & 0.344813 & -1.928825 & 2.358352 & $\mathrm{H}$ & -1.991062 & -1.500147 & -1.846317 \\
\hline $\mathrm{C}$ & 1.318857 & -1.950942 & -1.712228 & $\mathrm{H}$ & -5.502407 & -0.319724 & -0.883330 \\
\hline $\mathrm{C}$ & 1.412940 & -0.905958 & -2.838270 & $\mathrm{H}$ & -4.940258 & 0.488461 & 0.597680 \\
\hline $\mathrm{C}$ & 0.971464 & -3.316742 & -2.301633 & $\mathrm{H}$ & -2.694245 & 0.055044 & -2.299862 \\
\hline $\mathrm{H}$ & 2.118610 & -1.213955 & -3.616147 & $\mathrm{H}$ & -4.451208 & 1.105332 & -0.986317 \\
\hline $\mathrm{H}$ & 1.713695 & 0.072145 & -2.457293 & $\mathrm{H}$ & -5.553620 & -1.852934 & 1.272309 \\
\hline $\mathrm{H}$ & 1.799709 & -3.711250 & -2.900601 & $\mathrm{H}$ & -4.832195 & -3.161920 & -0.926690 \\
\hline $\mathrm{H}$ & 0.726411 & -4.034344 & -1.515947 & $\mathrm{C}$ & -0.301784 & 2.988213 & 2.793118 \\
\hline $\mathrm{C}$ & 2.582106 & -1.971758 & -0.786396 & $\mathrm{H}$ & 0.657583 & 2.930110 & 3.319495 \\
\hline $\mathrm{C}$ & 2.741606 & -3.258697 & 0.019917 & $\mathrm{H}$ & -0.313243 & 3.906385 & 2.196331 \\
\hline $\mathrm{C}$ & 3.876550 & -1.635049 & -1.524613 & $\mathrm{H}$ & -1.095190 & 3.084041 & 3.545718 \\
\hline $\mathrm{H}$ & 3.088307 & -4.073131 & -0.624255 & $\mathrm{~B}$ & 3.337622 & -0.041935 & 0.912804 \\
\hline $\mathrm{H}$ & 1.800640 & -3.554961 & 0.485747 & $\mathrm{~F}$ & 4.356206 & -0.901162 & 1.248036 \\
\hline $\mathrm{H}$ & 3.979545 & -2.322664 & -2.371422 & $\mathrm{~F}$ & 2.708583 & 0.494145 & 2.027972 \\
\hline $\mathrm{H}$ & 3.875943 & -0.610290 & -1.894677 & $\mathrm{~F}$ & 3.707635 & 0.944614 & 0.018946 \\
\hline
\end{tabular}

\section{TS-9b E = -1688.15876181}

$\mathrm{H} \quad 0.097456 \quad-3.216440 \quad-2.953358$

$\begin{array}{llll}\text { C } & 0.290394 & -0.578005 & 1.573192\end{array}$

$\mathrm{H} \quad 4.737851 \quad-1.758180 \quad-0.868252$

$\begin{array}{llll}\text { C } & -0.130317 & 0.558073 & 2.358460\end{array}$

$\mathrm{H} \quad 3.483956 \quad-3.087147 \quad 0.801553$

$\begin{array}{llll}\text { C } & -1.175024 & 1.426093 & 2.057590\end{array}$

$\begin{array}{llll}\mathrm{H} & 1.398700 & -1.799134 & 2.622366\end{array}$

$\begin{array}{llll}\text { O } & 2.075637 & -0.457463 & -0.535500\end{array}$

\section{SI-53}




\begin{tabular}{|c|c|c|c|}
\hline B & 1.289140 & 0.365663 & 0.425040 \\
\hline $\mathrm{O}$ & 0.296053 & 1.170379 & -0.298633 \\
\hline 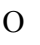 & 2.264216 & 1.142334 & 1.116518 \\
\hline $\mathrm{C}$ & -0.226692 & 2.205774 & 0.309786 \\
\hline $\mathrm{C}$ & -1.359629 & 2.855103 & -0.367101 \\
\hline$C$ & -2.151897 & 2.124202 & -1.269181 \\
\hline 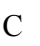 & -1.649659 & 4.204865 & -0.119480 \\
\hline $\mathrm{C}$ & -2.725021 & 4.819422 & -0.761426 \\
\hline $\mathrm{C}$ & -3.222692 & 2.743069 & -1.905319 \\
\hline 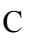 & -3.513954 & 4.088931 & -1.652162 \\
\hline$H$ & 0.561248 & 0.879027 & 3.141659 \\
\hline $\mathrm{H}$ & -1.990391 & 1.030525 & 1.460205 \\
\hline$U$ & 0.456071 & 2.835394 & 0.884082 \\
\hline $\mathrm{H}$ & -1.915188 & 1.080520 & -1.439675 \\
\hline $\mathrm{H}$ & -1.023472 & 4.774058 & 0.563255 \\
\hline$H$ & -2.942483 & 5.866619 & -0.570848 \\
\hline $\mathrm{H}$ & -3.834290 & 2.177427 & -2.603207 \\
\hline $\mathrm{H}$ & -4.351832 & 4.567115 & -2.152288 \\
\hline$C$ & 1.260870 & -1.528848 & 2.301215 \\
\hline 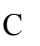 & 3.358368 & 0.259752 & -0.792690 \\
\hline$C$ & 3.090975 & 1.268129 & -1.912506 \\
\hline $\mathrm{C}$ & 4.445580 & -0.732413 & -1.194921 \\
\hline $\mathrm{H}$ & 4.013152 & 1.795249 & -2.176359 \\
\hline$H$ & 2.335698 & 2.001084 & -1.620176 \\
\hline $\mathrm{H}$ & 5.420359 & -0.237790 & -1.124857 \\
\hline $\mathrm{H}$ & 4.441828 & -1.608992 & -0.545928 \\
\hline$C$ & 3.587585 & 0.918373 & 0.610871 \\
\hline $\mathrm{C}$ & 4.330395 & -0.003355 & 1.589093 \\
\hline $\mathrm{C}$ & 4.305670 & 2.267426 & 0.538283 \\
\hline $\mathrm{H}$ & 5.392853 & -0.086044 & 1.338999 \\
\hline $\mathrm{H}$ & 3.897235 & -1.004654 & 1.603080 \\
\hline $\mathrm{H}$ & 5.293830 & 2.166003 & 0.075480 \\
\hline 11 & 3.723995 & 2.997874 & -0.028005 \\
\hline $\mathrm{H}$ & 2.729308 & 0.728270 & -2.788343 \\
\hline $\mathrm{H}$ & 4.296041 & -1.070176 & -2.219978 \\
\hline $\mathrm{H}$ & 4.442498 & 2.656049 & 1.552397 \\
\hline
\end{tabular}

\begin{tabular}{|c|c|c|c|}
\hline I & 4.243940 & 0.420891 & 2.594145 \\
\hline 4 & 1.775329 & -2.168759 & 1.580099 \\
\hline I & 2.004164 & -0.973172 & 2.880618 \\
\hline I & 0.708620 & -2.185500 & 2.981742 \\
\hline B & -0.882458 & -1.367758 & 0.863440 \\
\hline $\mathrm{O}$ & -1.934608 & -0.788789 & 0.199176 \\
\hline $\mathrm{O}$ & -1.024864 & -2.714155 & 1.038643 \\
\hline $\mathrm{C}$ & -2.196912 & -3.173562 & 0.319666 \\
\hline $\mathrm{C}$ & -1.700832 & -4.103509 & -0.792568 \\
\hline $\mathrm{C}$ & -3.044727 & -3.971299 & 1.314027 \\
\hline $\mathrm{H}$ & -1.168391 & -4.942529 & -0.334207 \\
\hline $\mathrm{H}$ & -1.003150 & -3.587942 & -1.451318 \\
\hline $\mathrm{H}$ & -2.465635 & -4.830903 & 1.664719 \\
\hline $\mathrm{H}$ & -3.314162 & -3.372412 & 2.187424 \\
\hline $\mathrm{C}$ & -2.877239 & -1.819624 & -0.196046 \\
\hline $\mathrm{C}$ & -3.033475 & -1.726841 & -1.716140 \\
\hline $\mathrm{C}$ & -4.203949 & -1.470286 & 0.485348 \\
\hline $\mathrm{H}$ & -3.692112 & -2.514059 & -2.097597 \\
\hline $\mathrm{H}$ & -2.062986 & -1.795322 & -2.208610 \\
\hline $\mathrm{H}$ & -4.988290 & -2.192846 & 0.237380 \\
\hline $\mathrm{H}$ & -4.095616 & -1.429258 & 1.572460 \\
\hline $\mathrm{H}$ & -3.479396 & -0.759755 & -1.972850 \\
\hline $\mathrm{H}$ & -4.525567 & -0.480865 & 0.144147 \\
\hline $\mathrm{H}$ & -3.961929 & -4.343657 & 0.845204 \\
\hline $\mathrm{H}$ & -2.534166 & -4.503994 & -1.379750 \\
\hline $\mathrm{C}$ & -1.507976 & 2.590416 & 2.957030 \\
\hline $\mathrm{H}$ & -0.615000 & 2.992041 & 3.450317 \\
\hline $\mathrm{H}$ & -1.987080 & 3.399281 & 2.395001 \\
\hline $\mathrm{H}$ & -2.214110 & 2.284919 & 3.739893 \\
\hline B & 1.588720 & -1.688867 & -1.427366 \\
\hline $\mathrm{F}$ & 2.084554 & -2.808503 & -0.805882 \\
\hline $\mathrm{F}$ & 0.204479 & -1.611515 & -1.392662 \\
\hline $\mathrm{F}$ & 2.082250 & -1.497613 & -2.696527 \\
\hline
\end{tabular}

TS-9c E $=\mathbf{- 1 6 8 8 . 1 3 1 3 1 1 5 3}$

$\begin{array}{llll}\text { C } & 0.870645 & 0.241363 & 1.759163\end{array}$ 


\begin{tabular}{|c|c|c|c|}
\hline $\mathrm{C}$ & -0.343814 & -0.335898 & 2.290544 \\
\hline $\mathrm{C}$ & -1.206117 & -1.277272 & 1.698887 \\
\hline $\mathrm{O}$ & 1.334013 & 1.745895 & -0.348207 \\
\hline B & 0.426233 & 1.358350 & 0.594647 \\
\hline $\mathrm{O}$ & -0.963730 & 0.159373 & -0.420999 \\
\hline $\mathrm{O}$ & -0.438368 & 2.370303 & 0.983067 \\
\hline $\mathrm{C}$ & -1.949219 & -0.058318 & 0.465724 \\
\hline $\mathrm{C}$ & -3.287583 & -0.638481 & 0.115011 \\
\hline $\mathrm{C}$ & -3.508044 & -1.793544 & -0.652790 \\
\hline $\mathrm{C}$ & -4.394483 & 0.036193 & 0.656725 \\
\hline $\mathrm{C}$ & -5.694012 & -0.408078 & 0.418547 \\
\hline $\mathrm{C}$ & -4.808195 & -2.234144 & -0.887817 \\
\hline $\mathrm{C}$ & -5.904052 & -1.547926 & -0.357581 \\
\hline $\mathrm{H}$ & -0.714016 & 0.114356 & 3.217730 \\
\hline $\mathrm{H}$ & -0.766750 & -1.928363 & 0.946234 \\
\hline $\mathrm{H}$ & -2.102418 & 0.845294 & 1.053788 \\
\hline $\mathrm{H}$ & -2.669333 & -2.337450 & -1.061533 \\
\hline $\mathrm{H}$ & -4.236192 & 0.927247 & 1.259706 \\
\hline $\mathrm{H}$ & -6.536457 & 0.135785 & 0.836801 \\
\hline $\mathrm{H}$ & -4.965417 & -3.124043 & -1.490942 \\
\hline $\mathrm{H}$ & -6.913996 & -1.901592 & -0.546936 \\
\hline $\mathrm{C}$ & 1.619898 & 1.114908 & 2.804851 \\
\hline $\mathrm{C}$ & 0.851985 & 2.999609 & -0.914702 \\
\hline $\mathrm{C}$ & 0.069646 & 2.678664 & -2.192385 \\
\hline $\mathrm{C}$ & 2.077421 & 3.844642 & -1.253367 \\
\hline $\mathrm{H}$ & -0.228052 & 3.600814 & -2.703293 \\
\hline $\mathrm{H}$ & -0.819926 & 2.081112 & -1.990161 \\
\hline $\mathrm{H}$ & 1.779506 & 4.838538 & -1.605775 \\
\hline $\mathrm{H}$ & 2.739522 & 3.958440 & -0.392218 \\
\hline $\mathrm{C}$ & -0.049146 & 3.570749 & 0.252583 \\
\hline $\mathrm{C}$ & 0.722509 & 4.444754 & 1.247156 \\
\hline $\mathrm{C}$ & -1.317589 & 4.283441 & -0.206548 \\
\hline $\mathrm{H}$ & 1.016265 & 5.400163 & 0.800729 \\
\hline $\mathrm{H}$ & 1.621286 & 3.933295 & 1.603015 \\
\hline $\mathrm{H}$ & -1.071049 & 5.161350 & -0.813973 \\
\hline $\mathrm{H}$ & -1.955278 & 3.619849 & -0.793713 \\
\hline
\end{tabular}

\begin{tabular}{|c|c|c|c|}
\hline $\mathrm{H}$ & 0.705406 & 2.090220 & -2.856369 \\
\hline $\mathrm{H}$ & 2.642555 & 3.353476 & -2.051288 \\
\hline $\mathrm{H}$ & -1.886033 & 4.620998 & 0.666403 \\
\hline H & 0.083251 & 4.647609 & 2.112256 \\
\hline $\mathrm{H}$ & 2.472867 & 1.609025 & 2.334385 \\
\hline H & 0.961786 & 1.881154 & 3.226940 \\
\hline $\mathrm{H}$ & 2.009723 & 0.495826 & 3.620879 \\
\hline B & 1.864643 & -0.734897 & 1.003652 \\
\hline $\mathrm{O}$ & 1.497375 & -1.910776 & 0.433280 \\
\hline $\mathrm{O}$ & 3.192146 & -0.438055 & 0.889393 \\
\hline $\mathrm{C}$ & 3.740378 & -1.346007 & -0.116542 \\
\hline $\mathrm{C}$ & 3.774755 & -0.556952 & -1.429418 \\
\hline $\mathrm{C}$ & 5.153182 & -1.731826 & 0.311193 \\
\hline $\mathrm{H}$ & 4.386971 & 0.338104 & -1.280953 \\
\hline $\mathrm{H}$ & 2.773739 & -0.238665 & -1.722201 \\
\hline $\mathrm{H}$ & 5.790128 & -0.841853 & 0.307939 \\
\hline $\mathrm{H}$ & 5.172274 & -2.157528 & 1.317035 \\
\hline $\mathrm{C}$ & 2.695862 & -2.536863 & -0.115233 \\
\hline $\mathrm{C}$ & 2.346301 & -3.087600 & -1.493952 \\
\hline $\mathrm{C}$ & 3.056848 & -3.670455 & 0.849504 \\
\hline $\mathrm{H}$ & 3.234804 & -3.515384 & -1.972508 \\
\hline $\mathrm{H}$ & 1.922800 & -2.309798 & -2.128010 \\
\hline $\mathrm{H}$ & 3.928537 & -4.233290 & 0.500573 \\
\hline $\mathrm{H}$ & 3.267429 & -3.284338 & 1.852105 \\
\hline $\mathrm{H}$ & 1.592419 & -3.873307 & -1.389959 \\
\hline $\mathrm{H}$ & 2.207297 & -4.356381 & 0.921247 \\
\hline $\mathrm{H}$ & 5.580711 & -2.460113 & -0.386946 \\
\hline $\mathrm{H}$ & 4.215716 & -1.152151 & -2.236000 \\
\hline $\mathrm{C}$ & -2.262218 & -1.914641 & 2.585458 \\
\hline $\mathrm{H}$ & -2.804145 & -1.165257 & 3.174243 \\
\hline $\mathrm{H}$ & -2.991942 & -2.465971 & 1.988914 \\
\hline $\mathrm{H}$ & -1.792304 & -2.619738 & 3.281153 \\
\hline B & -0.681058 & -0.710721 & -1.695814 \\
\hline $\mathrm{F}$ & 0.567753 & -0.315551 & -2.133363 \\
\hline $\mathrm{F}$ & -1.684202 & -0.418744 & -2.591529 \\
\hline $\mathrm{F}$ & -0.683774 & -2.057140 & -1.321117 \\
\hline
\end{tabular}




\begin{tabular}{|c|c|c|c|}
\hline \multicolumn{4}{|c|}{ TS-10a $E=-1688.15136507$} \\
\hline $\mathrm{C}$ & 0.350456 & -1.069371 & -1.084735 \\
\hline $\mathrm{C}$ & -0.459459 & -2.024538 & -0.371737 \\
\hline $\mathrm{C}$ & -1.797971 & -2.375735 & -0.586739 \\
\hline $\mathrm{O}$ & -0.102084 & 1.433095 & -1.670635 \\
\hline B & -0.343955 & 0.513359 & -0.631974 \\
\hline $\mathrm{O}$ & -1.790627 & 0.291549 & -0.443416 \\
\hline $\mathrm{O}$ & 0.268810 & 1.195137 & 0.592497 \\
\hline $\mathrm{C}$ & -2.314422 & -0.656078 & 0.317658 \\
\hline $\mathrm{C}$ & -3.794008 & -0.751741 & 0.287248 \\
\hline $\mathrm{C}$ & -4.543263 & -0.063073 & -0.676504 \\
\hline $\mathrm{C}$ & -4.452160 & -1.539710 & 1.242468 \\
\hline $\mathrm{C}$ & -5.840524 & -1.646552 & 1.228920 \\
\hline $\mathrm{C}$ & -5.933104 & -0.168695 & -0.685027 \\
\hline $\mathrm{C}$ & -6.584194 & -0.962656 & 0.262419 \\
\hline $\mathrm{H}$ & -0.066545 & -2.333021 & 0.592463 \\
\hline $\mathrm{H}$ & -1.871209 & -0.806541 & 1.299789 \\
\hline $\mathrm{H}$ & -4.025768 & 0.560834 & -1.397113 \\
\hline $\mathrm{H}$ & -3.870732 & -2.064191 & 1.997366 \\
\hline $\mathrm{H}$ & -6.343741 & -2.256138 & 1.974183 \\
\hline $\mathrm{H}$ & -6.509978 & 0.372568 & -1.429928 \\
\hline $\mathrm{H}$ & -7.667771 & -1.042775 & 0.253567 \\
\hline $\mathrm{C}$ & 0.305185 & -1.074675 & -2.638708 \\
\hline $\mathrm{C}$ & -0.085344 & 2.761966 & -1.143689 \\
\hline $\mathrm{C}$ & -1.534639 & 3.267034 & -1.012908 \\
\hline $\mathrm{C}$ & 0.672814 & 3.646502 & -2.133244 \\
\hline $\mathrm{H}$ & -1.568405 & 4.326080 & -0.738021 \\
\hline $\mathrm{H}$ & -2.100235 & 2.693514 & -0.276234 \\
\hline $\mathrm{H}$ & 0.812316 & 4.657394 & -1.734060 \\
\hline $\mathrm{H}$ & 1.648469 & 3.220411 & -2.374898 \\
\hline $\mathrm{C}$ & 0.641665 & 2.604848 & 0.237868 \\
\hline $\mathrm{C}$ & 2.163395 & 2.632821 & 0.134535 \\
\hline $\mathrm{C}$ & 0.149243 & 3.585400 & 1.300167 \\
\hline $\mathrm{H}$ & 2.510768 & 3.647342 & -0.086169 \\
\hline $\mathrm{H}$ & 2.514163 & 1.949093 & -0.638256 \\
\hline $\mathrm{H}$ & 0.261452 & 4.602843 & 0.909378 \\
\hline
\end{tabular}

\begin{tabular}{|c|c|c|c|}
\hline $\mathrm{H}$ & -0.895965 & 3.415175 & 1.556950 \\
\hline $\mathrm{H}$ & -2.024035 & 3.144210 & -1.983958 \\
\hline $\mathrm{H}$ & 0.096677 & 3.721184 & -3.060768 \\
\hline $\mathrm{H}$ & 0.741564 & 3.495692 & 2.210919 \\
\hline $\mathrm{H}$ & 2.581877 & 2.325335 & 1.094711 \\
\hline $\mathrm{H}$ & 1.071579 & -0.394851 & -3.013739 \\
\hline F & 0.514211 & -2.080023 & -3.023515 \\
\hline $\mathrm{H}$ & -0.646806 & -0.737130 & -3.048496 \\
\hline B & 1.845094 & -1.129548 & -0.580374 \\
\hline $\mathrm{O}$ & 2.842034 & -0.312019 & -1.049939 \\
\hline $\mathrm{O}$ & 2.297691 & -2.077507 & 0.300324 \\
\hline C & 3.665316 & -1.728842 & 0.656080 \\
\hline$C$ & 3.594949 & -0.948404 & 1.971790 \\
\hline $\mathrm{C}$ & 4.456327 & -3.019703 & 0.846012 \\
\hline $\mathrm{H}$ & 3.077510 & -1.561100 & 2.715159 \\
\hline $\mathrm{H}$ & 3.022793 & -0.025223 & 1.868727 \\
\hline $\mathrm{H}$ & 4.064810 & -3.563999 & 1.710941 \\
\hline $\mathrm{H}$ & 4.381275 & -3.672302 & -0.026671 \\
\hline $\mathrm{C}$ & 4.109913 & -0.855624 & -0.577357 \\
\hline $\mathrm{C}$ & 5.046590 & 0.299287 & -0.239992 \\
\hline $\mathrm{C}$ & 4.677060 & -1.681863 & -1.737288 \\
\hline $\mathrm{H}$ & 5.990546 & -0.081342 & 0.166043 \\
\hline $\mathrm{H}$ & 4.601081 & 0.977694 & 0.488879 \\
\hline $\mathrm{H}$ & 5.663122 & -2.092135 & -1.496905 \\
\hline $\mathrm{H}$ & 4.006485 & -2.508319 & -1.992879 \\
\hline $\mathrm{H}$ & 5.269855 & 0.870994 & -1.146547 \\
\hline $\mathrm{H}$ & 4.771797 & -1.038183 & -2.617058 \\
\hline $\mathrm{H}$ & 5.513761 & -2.799800 & 1.031456 \\
\hline $\mathrm{H}$ & 4.596263 & -0.710645 & 2.346493 \\
\hline B & 0.166916 & 0.728026 & 2.090289 \\
\hline $\mathrm{F}$ & -1.062771 & 1.136013 & 2.563445 \\
\hline$F$ & 1.233602 & 1.289722 & 2.761916 \\
\hline$F$ & 0.260337 & -0.657419 & 2.032036 \\
\hline $\mathrm{H}$ & -2.186842 & -3.105880 & 0.123325 \\
\hline $\mathrm{C}$ & -2.520458 & -2.356492 & -1.917270 \\
\hline $\mathrm{H}$ & -2.526311 & -1.357400 & -2.357817 \\
\hline
\end{tabular}




\begin{tabular}{|c|c|c|c|}
\hline $\mathrm{H}$ & -2.037464 & -3.036323 & -2.627120 \\
\hline $\mathrm{H}$ & -3.558128 & -2.673740 & -1.786920 \\
\hline \multicolumn{4}{|c|}{ TS-10b $E=-1688.14184876$} \\
\hline $\mathrm{C}$ & -0.185962 & -1.281063 & 0.248509 \\
\hline $\mathrm{C}$ & 0.516389 & -1.909329 & -0.833438 \\
\hline $\mathrm{C}$ & 1.833065 & -2.379590 & -0.868988 \\
\hline $\mathrm{O}$ & -0.290765 & 1.513592 & 0.496276 \\
\hline $\mathrm{B}$ & 0.336648 & 0.398430 & -0.260546 \\
\hline $\mathrm{O}$ & 1.781482 & 0.315051 & 0.026663 \\
\hline $\mathrm{O}$ & 0.065879 & 0.668636 & -1.625873 \\
\hline $\mathrm{C}$ & 2.540768 & -0.338186 & -0.821081 \\
\hline $\mathrm{C}$ & 3.942218 & -0.522224 & -0.416500 \\
\hline $\mathrm{C}$ & 4.333547 & -0.267581 & 0.907818 \\
\hline $\mathrm{C}$ & 4.891313 & -0.961414 & -1.351468 \\
\hline $\mathrm{C}$ & 6.217312 & -1.148253 & -0.968211 \\
\hline $\mathrm{C}$ & 5.661454 & -0.453240 & 1.284583 \\
\hline $\mathrm{C}$ & 6.603882 & -0.896214 & 0.351646 \\
\hline $\mathrm{H}$ & 0.056417 & -1.763541 & -1.810050 \\
\hline $\mathrm{H}$ & 2.332328 & -0.186012 & -1.881048 \\
\hline $\mathrm{H}$ & 3.590337 & 0.088250 & 1.615027 \\
\hline $\mathrm{H}$ & 4.584252 & -1.158651 & -2.376234 \\
\hline $\mathrm{H}$ & 6.949437 & -1.486965 & -1.695929 \\
\hline $\mathrm{H}$ & 5.963660 & -0.250744 & 2.308246 \\
\hline $\mathrm{H}$ & 7.638261 & -1.041762 & 0.650800 \\
\hline $\mathrm{C}$ & 0.220263 & -1.630456 & 1.693986 \\
\hline $\mathrm{C}$ & -0.316156 & 2.691027 & -0.424488 \\
\hline $\mathrm{C}$ & 1.024655 & 3.415574 & -0.277081 \\
\hline $\mathrm{C}$ & -1.475028 & 3.619985 & -0.071056 \\
\hline $\mathrm{H}$ & 1.049442 & 4.301471 & -0.919185 \\
\hline $\mathrm{H}$ & 1.864221 & 2.766938 & -0.535431 \\
\hline $\mathrm{H}$ & -1.632669 & 4.317513 & -0.901054 \\
\hline $\mathrm{H}$ & -2.397529 & 3.059354 & 0.091797 \\
\hline $\mathrm{C}$ & -0.507896 & 1.968340 & -1.810210 \\
\hline $\mathrm{C}$ & -1.978199 & 1.770872 & -2.195307 \\
\hline $\mathrm{C}$ & 0.230065 & 2.659868 & -2.959460 \\
\hline
\end{tabular}

\begin{tabular}{|c|c|c|c|}
\hline$H$ & -2.458499 & 2.711814 & -2.481772 \\
\hline 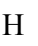 & -2.532386 & 1.313269 & -1.378602 \\
\hline $\mathrm{H}$ & -0.128417 & 3.685775 & -3.101147 \\
\hline F & 1.308185 & 2.684721 & -2.786101 \\
\hline $\mathrm{H}$ & 1.139289 & 3.732894 & 0.760266 \\
\hline H & -1.258804 & 4.189545 & 0.829995 \\
\hline $\mathrm{H}$ & 0.047759 & 2.104254 & -3.884704 \\
\hline $\mathrm{H}$ & -2.021297 & 1.078461 & -3.040001 \\
\hline $\mathrm{H}$ & -0.478624 & -1.179326 & 2.395037 \\
\hline $\mathrm{H}$ & 0.189219 & -2.716457 & 1.838588 \\
\hline $\mathrm{H}$ & 1.212465 & -1.264528 & 1.948888 \\
\hline B & -1.751743 & -1.290832 & 0.079255 \\
\hline $\mathrm{O}$ & -2.573194 & -1.523387 & 1.149821 \\
\hline $\mathrm{O}$ & -2.436436 & -1.146576 & -1.105071 \\
\hline $\mathrm{C}$ & -3.825362 & -1.505102 & -0.834058 \\
\hline $\mathrm{C}$ & -4.745198 & -0.644347 & -1.692916 \\
\hline $\mathrm{C}$ & -3.972067 & -2.982072 & -1.219494 \\
\hline $\mathrm{H}$ & -4.553596 & -0.842203 & -2.752368 \\
\hline $\mathrm{H}$ & -4.594565 & 0.421315 & -1.515467 \\
\hline $\mathrm{H}$ & -3.675701 & -3.106126 & -2.265597 \\
\hline $\mathrm{H}$ & -3.322842 & -3.612465 & -0.603863 \\
\hline $\mathrm{C}$ & -3.932109 & -1.256734 & 0.714279 \\
\hline $\mathrm{C}$ & -4.227922 & 0.201188 & 1.086500 \\
\hline $\mathrm{C}$ & -4.876263 & -2.196966 & 1.456028 \\
\hline $\mathrm{H}$ & -5.267123 & 0.469918 & 0.869902 \\
\hline $\mathrm{H}$ & -3.562207 & 0.886122 & 0.554769 \\
\hline $\mathrm{H}$ & -5.901907 & -2.084484 & 1.086442 \\
\hline $\mathrm{H}$ & -4.574212 & -3.240571 & 1.343609 \\
\hline $\mathrm{H}$ & -4.032341 & 0.336839 & 2.152746 \\
\hline $\mathrm{H}$ & -4.868302 & -1.955690 & 2.523287 \\
\hline $\mathrm{H}$ & -5.004492 & -3.328083 & -1.106276 \\
\hline $\mathrm{H}$ & -5.793318 & -0.886263 & -1.483235 \\
\hline B & -0.282964 & 1.643277 & 2.137037 \\
\hline F & -1.416184 & 0.964718 & 2.512715 \\
\hline $\mathrm{F}$ & 0.889148 & 1.057378 & 2.541923 \\
\hline $\mathrm{F}$ & -0.326227 & 2.986310 & 2.420819 \\
\hline
\end{tabular}




$$
\begin{array}{llll}
\mathrm{H} & 2.165169 & -2.668750 & -1.867838 \\
\mathrm{C} & 2.591596 & -3.104406 & 0.224198 \\
\mathrm{H} & 2.573855 & -2.589909 & 1.183075 \\
\mathrm{H} & 2.164726 & -4.104670 & 0.369089 \\
\mathrm{H} & 3.637555 & -3.227889 & -0.070772
\end{array}
$$

\begin{tabular}{|c|c|c|c|}
\hline $\mathrm{C}$ & -0.618737 & -0.104186 & -1.526232 \\
\hline $\mathrm{C}$ & 0.457800 & -0.831641 & -2.159889 \\
\hline $\mathrm{C}$ & 1.259573 & -1.877828 & -1.695781 \\
\hline $\mathrm{O}$ & -0.841817 & 0.642470 & 1.000227 \\
\hline B & 0.092695 & 0.640736 & -0.068246 \\
\hline $\mathrm{O}$ & 1.296236 & -0.110972 & 0.316907 \\
\hline $\mathrm{O}$ & 0.399881 & 2.114181 & -0.270367 \\
\hline $\mathrm{C}$ & 2.251749 & -0.505302 & -0.499450 \\
\hline $\mathrm{C}$ & 3.303782 & -1.354858 & 0.092270 \\
\hline $\mathrm{C}$ & 3.163963 & -1.890524 & 1.381040 \\
\hline $\mathrm{C}$ & 4.467499 & -1.619177 & -0.645525 \\
\hline $\mathrm{C}$ & 5.474100 & -2.416054 & -0.106977 \\
\hline $\mathrm{C}$ & 4.175579 & -2.683241 & 1.918858 \\
\hline $\mathrm{C}$ & 5.328925 & -2.951337 & 1.176586 \\
\hline $\mathrm{H}$ & 0.850019 & -0.350253 & -3.056451 \\
\hline $\mathrm{H}$ & 2.584589 & 0.193743 & -1.261096 \\
\hline $\mathrm{H}$ & 2.267533 & -1.665180 & 1.948265 \\
\hline $\mathrm{H}$ & 4.578627 & -1.194839 & -1.640737 \\
\hline $\mathrm{H}$ & 6.373757 & -2.614854 & -0.682502 \\
\hline $\mathrm{H}$ & 4.066521 & -3.090831 & 2.920121 \\
\hline $\mathrm{H}$ & 6.116180 & -3.569756 & 1.599014 \\
\hline $\mathrm{C}$ & -1.174433 & 0.986738 & -2.481585 \\
\hline $\mathrm{C}$ & -0.653109 & 1.811115 & 1.805594 \\
\hline $\mathrm{C}$ & 0.518743 & 1.569455 & 2.772624 \\
\hline $\mathrm{C}$ & -1.939756 & 2.056717 & 2.591422 \\
\hline $\mathrm{H}$ & 0.613326 & 2.382196 & 3.499813 \\
\hline $\mathrm{H}$ & 1.464547 & 1.464778 & 2.236430 \\
\hline $\mathrm{H}$ & -1.899450 & 3.011438 & 3.127250 \\
\hline $\mathrm{H}$ & -2.809665 & 2.055099 & 1.931171 \\
\hline
\end{tabular}

\begin{tabular}{|c|c|c|c|}
\hline $\mathrm{C}$ & -0.344610 & 2.918708 & 0.747757 \\
\hline $\mathrm{C}$ & -1.590026 & 3.455301 & 0.046265 \\
\hline$C$ & 0.508095 & 4.062282 & 1.291280 \\
\hline $\mathrm{H}$ & -2.120564 & 4.158916 & 0.696086 \\
\hline $\mathrm{H}$ & -2.270681 & 2.644697 & -0.222544 \\
\hline $\mathrm{H}$ & 0.005797 & 4.475753 & 2.172933 \\
\hline $\mathrm{H}$ & 1.505245 & 3.726213 & 1.572896 \\
\hline $\mathrm{H}$ & 0.328591 & 0.637480 & 3.313762 \\
\hline $\mathrm{H}$ & -2.070949 & 1.258503 & 3.329301 \\
\hline $\mathrm{H}$ & 0.613610 & 4.851123 & 0.546462 \\
\hline $\mathrm{H}$ & -1.276738 & 3.979592 & -0.859313 \\
\hline $\mathrm{H}$ & -1.898696 & 1.623462 & -1.977892 \\
\hline $\mathrm{H}$ & -0.375367 & 1.615792 & -2.873937 \\
\hline $\mathrm{H}$ & -1.697431 & 0.517511 & -3.323932 \\
\hline B & -1.839106 & -0.853838 & -0.851632 \\
\hline $\mathrm{O}$ & -1.861359 & -2.126786 & -0.341557 \\
\hline $\mathrm{O}$ & -3.057427 & -0.224532 & -0.765809 \\
\hline $\mathrm{C}$ & -4.022732 & -1.179103 & -0.255650 \\
\hline $\mathrm{C}$ & -4.945932 & -0.450058 & 0.716571 \\
\hline $\mathrm{C}$ & -4.813589 & -1.686805 & -1.466819 \\
\hline $\mathrm{H}$ & -5.521113 & 0.308638 & 0.176416 \\
\hline $\mathrm{H}$ & -4.375841 & 0.051840 & 1.501018 \\
\hline $\mathrm{H}$ & -5.267071 & -0.831045 & -1.975912 \\
\hline $\mathrm{H}$ & -4.153986 & -2.192088 & -2.179239 \\
\hline $\mathrm{C}$ & -3.104581 & -2.280279 & 0.401061 \\
\hline $\mathrm{C}$ & -2.769205 & -2.003737 & 1.871371 \\
\hline $\mathrm{C}$ & -3.591576 & -3.715775 & 0.227895 \\
\hline $\mathrm{H}$ & -3.649452 & -2.117846 & 2.512563 \\
\hline $\mathrm{H}$ & -2.354891 & -1.000276 & 1.988597 \\
\hline $\mathrm{H}$ & -4.561557 & -3.856116 & 0.718129 \\
\hline $\mathrm{H}$ & -3.689506 & -3.985260 & -0.825951 \\
\hline $\mathrm{H}$ & -2.008838 & -2.720560 & 2.197759 \\
\hline $\mathrm{H}$ & -2.874656 & -4.403356 & 0.687618 \\
\hline $\mathrm{H}$ & -5.608707 & -2.379409 & -1.172044 \\
\hline $\mathrm{H}$ & -5.651162 & -1.147591 & 1.182501 \\
\hline B & 1.584084 & 2.727253 & -1.121580 \\
\hline
\end{tabular}

\section{TS-11a $E=-\mathbf{1 6 8 8 . 1 5 1 8 8 1 7 1}$} SI-58 


$\begin{array}{llll}\text { F } & 1.167189 & 3.969187 & -1.533930 \\ \text { F } & 1.749139 & 1.844452 & -2.188460 \\ \text { F } & 2.692484 & 2.740493 & -0.303725 \\ \text { H } & 2.047086 & -2.168299 & -2.391592 \\ \text { C } & 0.870696 & -2.956492 & -0.714712 \\ \text { H } & 0.441962 & -2.538534 & 0.196572 \\ \text { H } & 0.108456 & -3.606727 & -1.153712 \\ \text { H } & 1.745377 & -3.560304 & -0.458559\end{array}$

\section{TS-11b E = -1688.14517098}

\begin{tabular}{|c|c|c|c|}
\hline C & 0.238902 & -0.564536 & 1.585697 \\
\hline $\mathrm{C}$ & -0.082541 & 0.584496 & 2.393742 \\
\hline C & -1.078823 & 1.556784 & 2.251253 \\
\hline C & 2.085993 & -0.387418 & -0.513269 \\
\hline B & 1.276339 & 0.409318 & 0.457088 \\
\hline $\mathrm{O}$ & 0.278252 & 1.231395 & -0.235403 \\
\hline $\mathrm{O}$ & 2.243797 & 1.175976 & 1.171969 \\
\hline C & -0.198391 & 2.275432 & 0.404773 \\
\hline C & -1.333268 & 2.961559 & -0.223471 \\
\hline $\mathrm{C}$ & -2.115594 & 2.303705 & -1.187226 \\
\hline C & -1.651757 & 4.274397 & 0.155194 \\
\hline C & -2.735398 & 4.927898 & -0.427384 \\
\hline $\mathrm{C}$ & -3.196609 & 2.962742 & -1.766512 \\
\hline $\mathrm{C}$ & -3.509461 & 4.272640 & -1.389678 \\
\hline $\mathrm{H}$ & 0.727270 & 0.878857 & 3.063124 \\
\hline $\mathrm{H}$ & 0.527022 & 2.880140 & 0.949979 \\
\hline $\mathrm{H}$ & -1.869237 & 1.280246 & -1.447455 \\
\hline $\mathrm{H}$ & -1.047466 & 4.779168 & 0.905811 \\
\hline $\mathrm{H}$ & -2.975510 & 5.946301 & -0.134977 \\
\hline $\mathrm{H}$ & -3.800128 & 2.453673 & -2.513134 \\
\hline $\mathrm{H}$ & -4.354883 & 4.782239 & -1.843940 \\
\hline $\mathrm{C}$ & 1.210666 & -1.547316 & 2.276788 \\
\hline $\mathrm{C}$ & 3.350949 & 0.377194 & -0.756944 \\
\hline $\mathrm{C}$ & 3.032093 & 1.406596 & -1.843295 \\
\hline $\mathrm{C}$ & 4.483163 & -0.542251 & -1.206586 \\
\hline $\mathrm{H}$ & 3.934586 & 1.961945 & -2.117110 \\
\hline
\end{tabular}

\begin{tabular}{|c|c|c|c|}
\hline $\mathrm{H}$ & 2.265766 & 2.113926 & -1.519958 \\
\hline $\mathrm{H}$ & 5.422344 & 0.018551 & -1.145135 \\
\hline $\mathrm{H}$ & 4.556119 & -1.431789 & -0.582571 \\
\hline $\mathrm{C}$ & 3.570364 & 0.986669 & 0.664615 \\
\hline $\mathrm{C}$ & 4.313158 & 0.032328 & 1.610714 \\
\hline $\mathrm{C}$ & 4.273192 & 2.344608 & 0.640306 \\
\hline $\mathrm{H}$ & 5.377784 & -0.035589 & 1.365831 \\
\hline $\mathrm{H}$ & 3.882645 & -0.970383 & 1.578603 \\
\hline $\mathrm{H}$ & 5.265182 & 2.268257 & 0.181099 \\
\hline $\mathrm{H}$ & 3.686845 & 3.085344 & 0.091972 \\
\hline $\mathrm{H}$ & 2.663766 & 0.873410 & -2.721514 \\
\hline $\mathrm{H}$ & 4.333600 & -0.862458 & -2.237346 \\
\hline $\mathrm{H}$ & 4.399234 & 2.703464 & 1.666701 \\
\hline $\mathrm{H}$ & 4.216700 & 0.415626 & 2.631205 \\
\hline $\mathrm{H}$ & 1.715371 & -2.176278 & 1.539586 \\
\hline $\mathrm{H}$ & 1.966680 & -1.014072 & 2.861374 \\
\hline $\mathrm{H}$ & 0.656576 & -2.211073 & 2.948082 \\
\hline B & -0.920075 & -1.349285 & 0.834400 \\
\hline $\mathrm{O}$ & -1.821926 & -0.837009 & -0.061998 \\
\hline $\mathrm{O}$ & -1.167050 & -2.654562 & 1.159666 \\
\hline $\mathrm{C}$ & -2.233321 & -3.170369 & 0.323718 \\
\hline $\mathrm{C}$ & -1.615404 & -4.246927 & -0.575094 \\
\hline $\mathrm{C}$ & -3.261287 & -3.813698 & 1.258306 \\
\hline $\mathrm{H}$ & -1.205920 & -5.039697 & 0.058768 \\
\hline $\mathrm{H}$ & -0.798926 & -3.838895 & -1.169616 \\
\hline $\mathrm{H}$ & -2.784720 & -4.638525 & 1.796717 \\
\hline $\mathrm{H}$ & -3.634321 & -3.103399 & 2.000085 \\
\hline $\mathrm{C}$ & -2.757831 & -1.875587 & -0.454046 \\
\hline $\mathrm{C}$ & -2.699575 & -1.977270 & -1.980155 \\
\hline $\mathrm{C}$ & -4.150601 & -1.400320 & -0.024850 \\
\hline $\mathrm{H}$ & -3.355001 & -2.772872 & -2.350180 \\
\hline $\mathrm{H}$ & -1.679926 & -2.157095 & -2.319641 \\
\hline $\mathrm{H}$ & -4.923202 & -2.122815 & -0.307102 \\
\hline $\mathrm{H}$ & -4.207178 & -1.232643 & 1.053571 \\
\hline $\mathrm{H}$ & -3.037617 & -1.029841 & -2.414050 \\
\hline $\mathrm{H}$ & -4.370968 & -0.450005 & -0.522107 \\
\hline
\end{tabular}




$\begin{array}{lrrr}\text { H } & -4.111271 & -4.216504 & 0.697115 \\ \text { H } & -2.365433 & -4.690593 & -1.238488 \\ \text { B } & 1.764695 & -1.708353 & -1.341360 \\ \text { F } & 2.488367 & -2.711639 & -0.738208 \\ \text { F } & 0.399255 & -1.883250 & -1.207862 \\ \text { F } & 2.133416 & -1.463821 & -2.643017 \\ \text { H } & -0.916299 & 2.424163 & 2.894225 \\ \text { C } & -2.543203 & 1.338576 & 1.942079 \\ \text { H } & -2.699921 & 0.733339 & 1.054566 \\ \text { H } & -3.018349 & 0.833862 & 2.793982 \\ \text { H } & -3.044404 & 2.300849 & 1.803697\end{array}$




\section{Reference:}

1. (a) Xu, Y.; Young, M. C.; Dong, G. J. Am. Chem. Soc. 2017, 139, 5716. (b) Hayashi, T.; Yamasaki, K. Chem. Rev. 2003, 103, 2829. (c) Sakai, M.; Hayashi, H.; Miyaura, N. Organometallics 1997, 16, 4229.

2. Sabitha, G.; Gopal, P.; Yadav, J. S. Tetrahedron: Asymmetry 2009, 20, 2205.

3. (a) Nishimaru, T.; Eto, K.; Komine, K.; Ishihara, J.; Hatakeyama, S. Chem. Eur. J. 2019, 25, 7927. (b) Wang, Z.; Moloney, M. G. Tetrahedron Lett. 2002, 43, 9629.

4. (a) Dale, J. A.; Mosher, H. S. J. Am. Chem. Soc. 1973, 95, 512. (b) Ohtani, I.; Kusumi, T.; Kashman, Y.; Kakisawa, H. J. Am. Chem. Soc. 1991, 113, 4092. (c) Hoye, T. R.; Jeffrey; C. S.; Shao, F. Nat. Protoc. 2007, 2, 2451. 

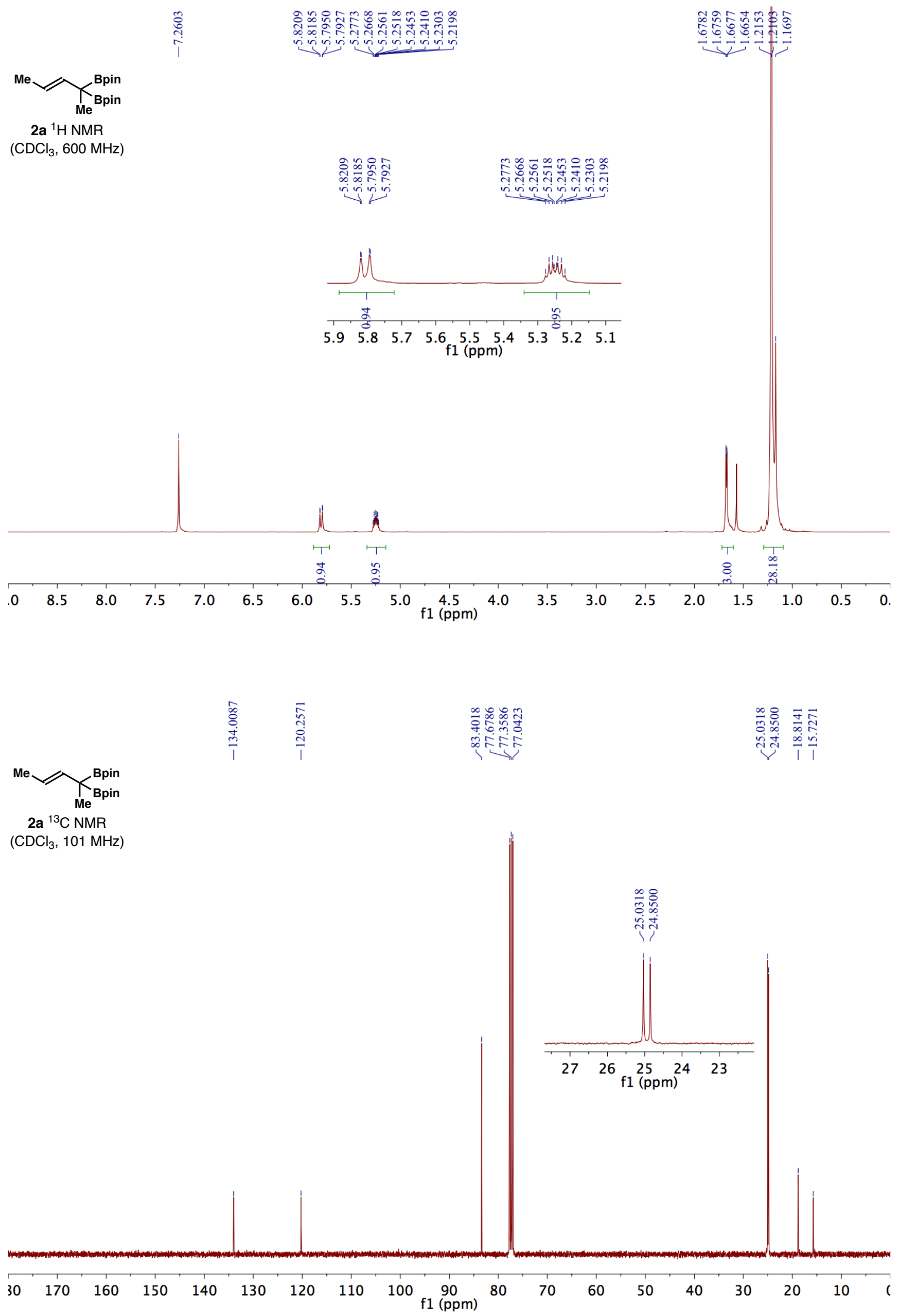

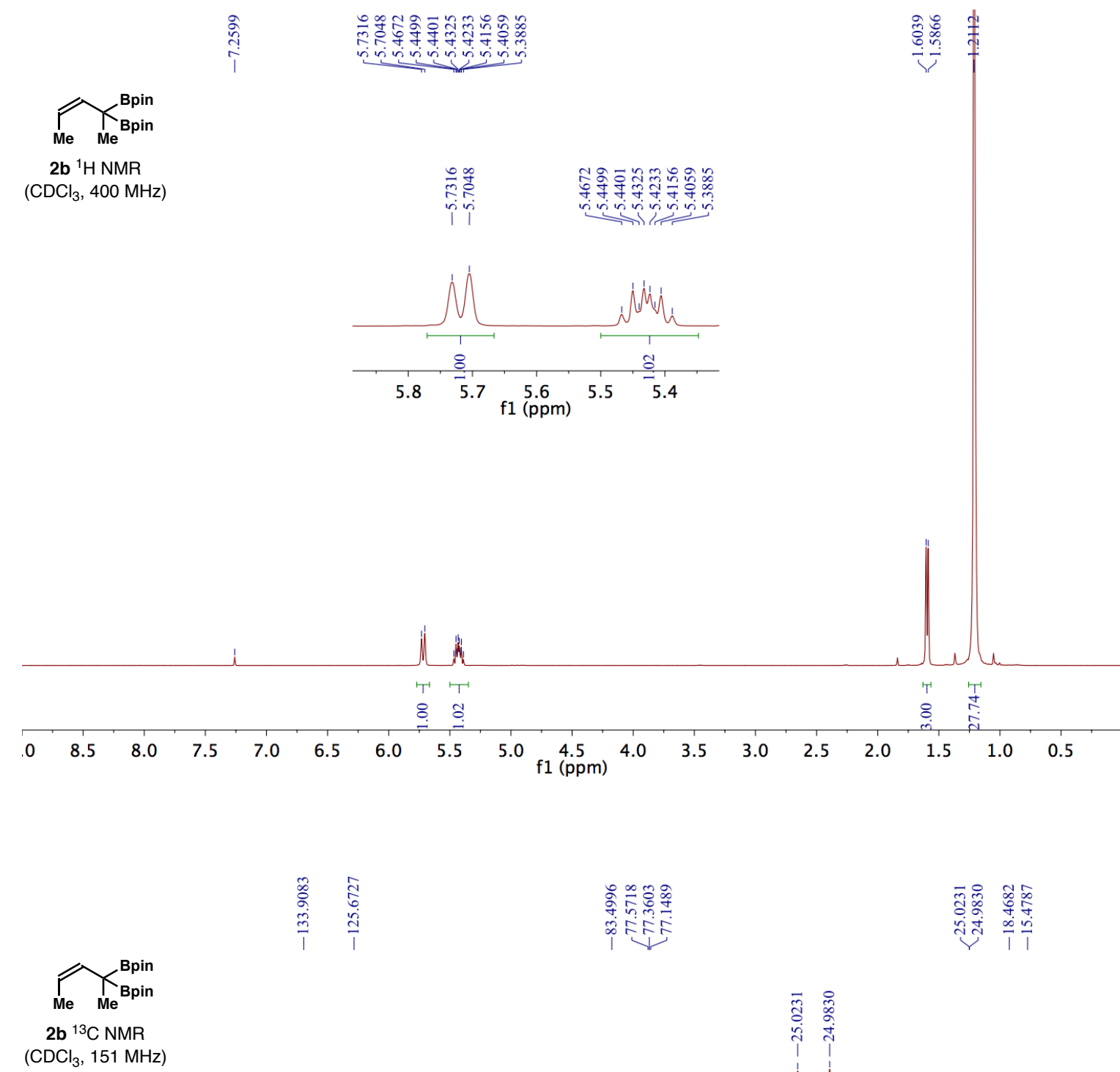

\section{$\left(\mathrm{CDCl}_{3}, 151 \mathrm{MHz}\right.$}
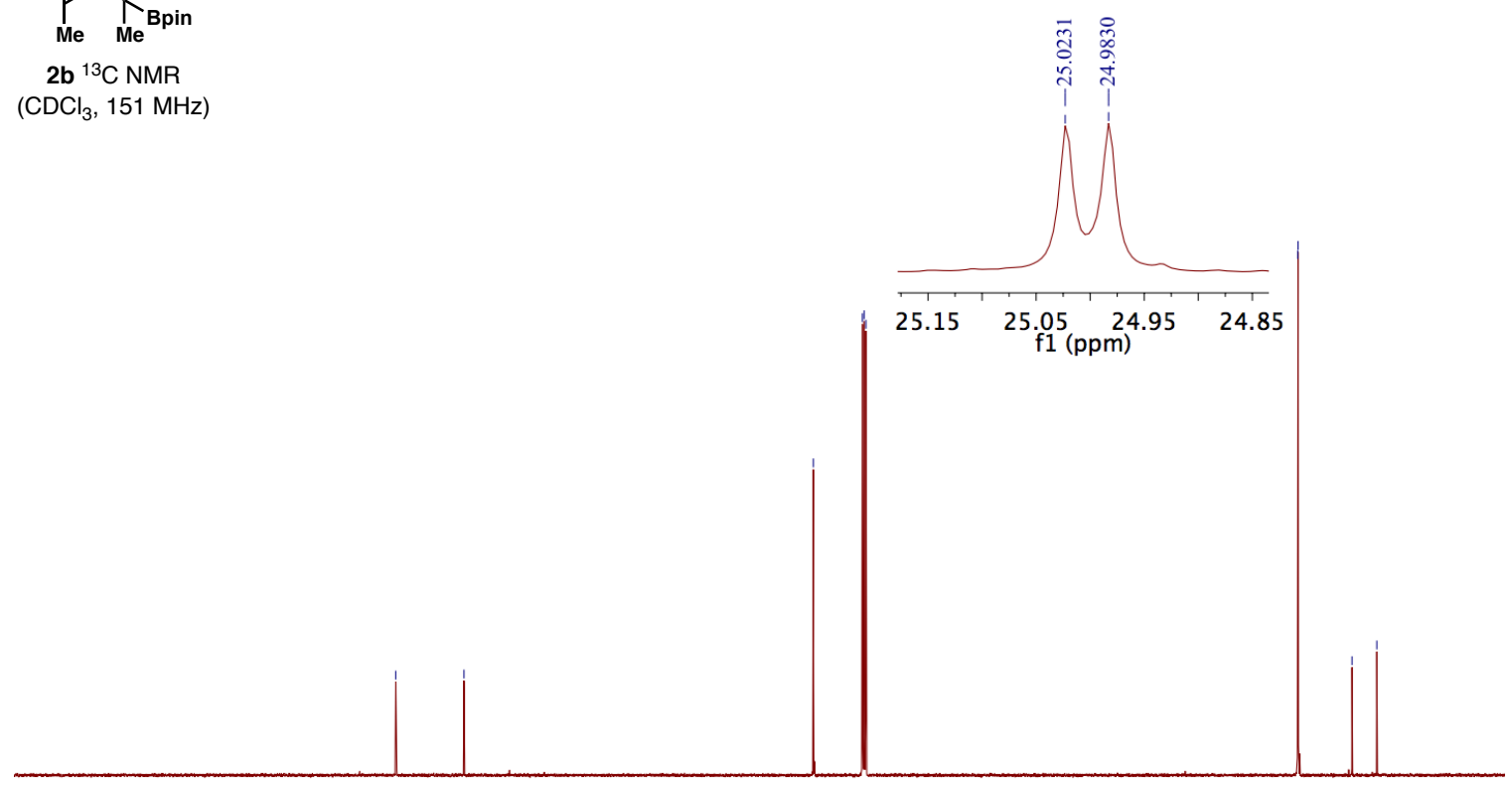

$\begin{array}{llllllllllllllllll}30 & 170 & 160 & 150 & 140 & 130 & 120 & 110 & 100 \underset{\mathrm{f} 1(\mathrm{ppm})}{90} & 80 & 70 & 60 & 50 & 40 & 30 & 20 & 10 & (\end{array}$ 

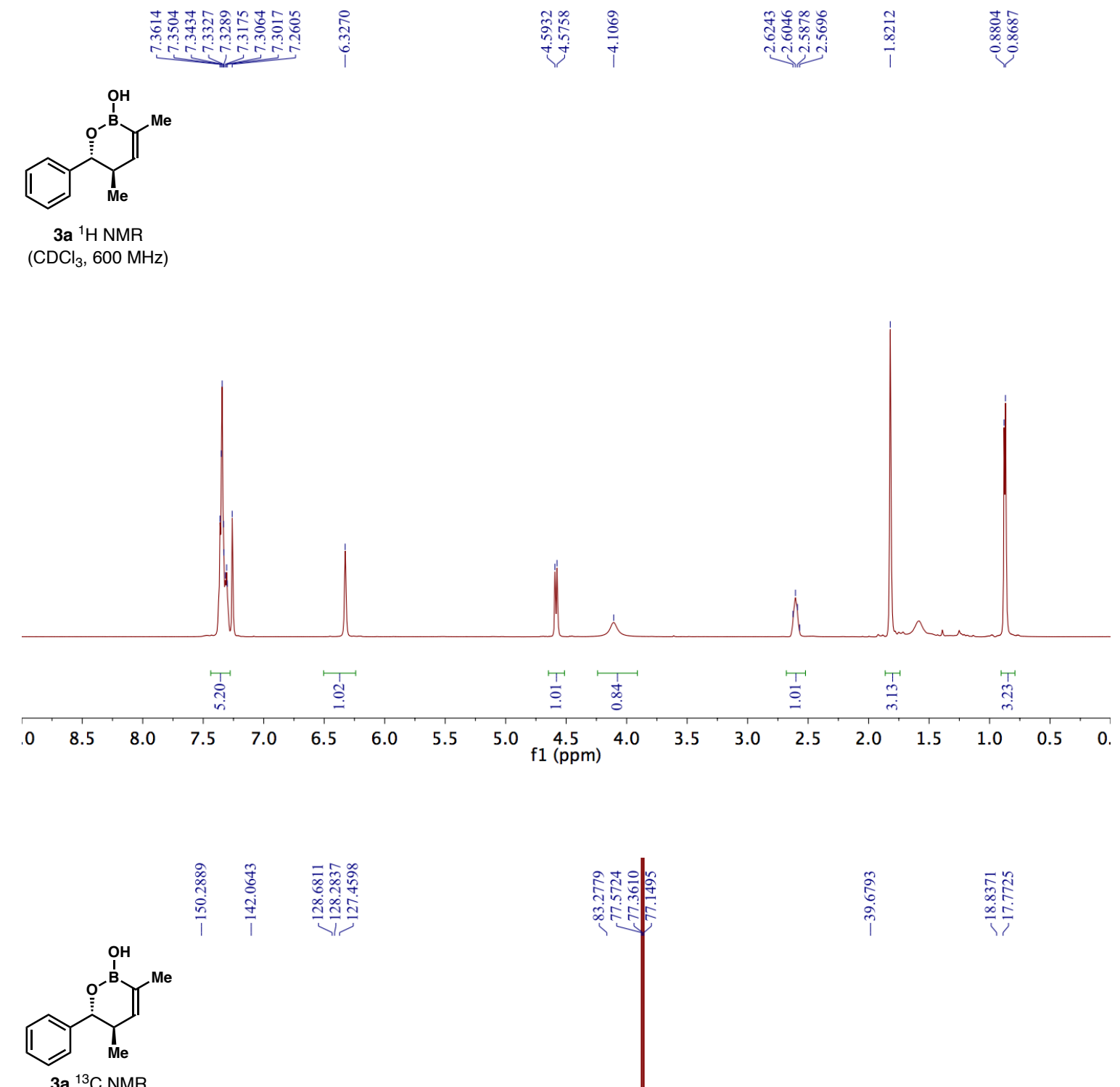

$\left(\mathrm{CDCl}_{3}, 151 \mathrm{MHz}\right)$

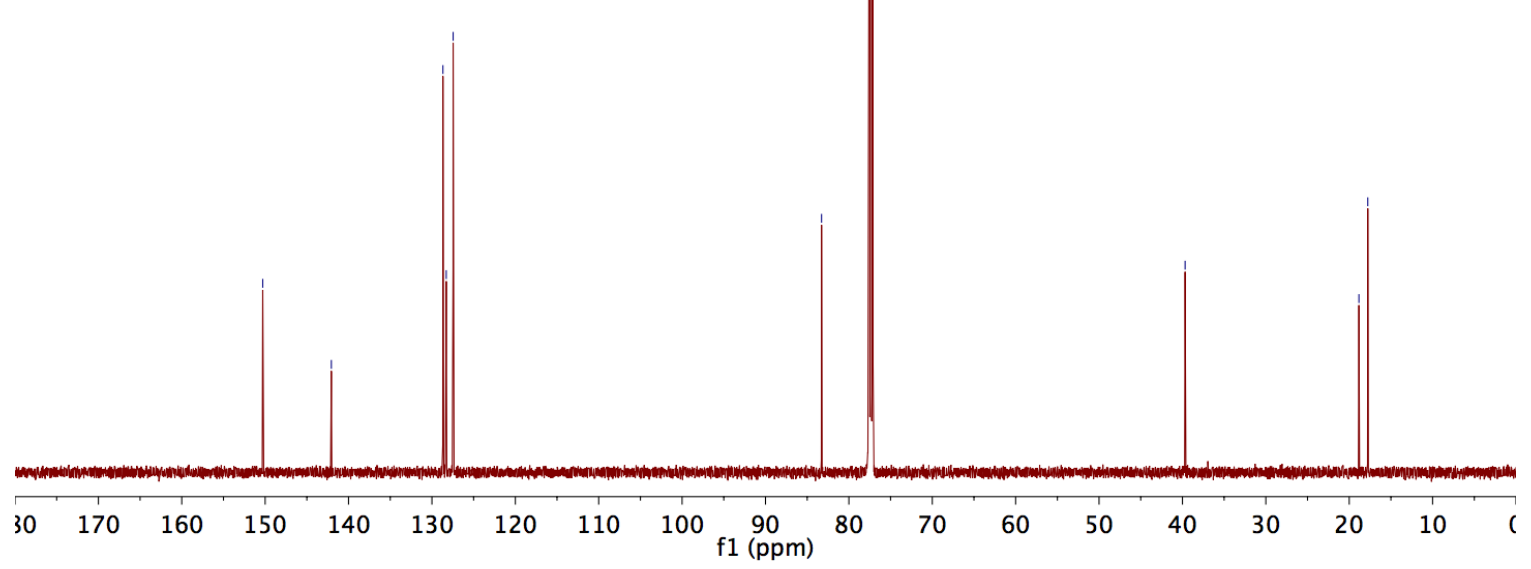



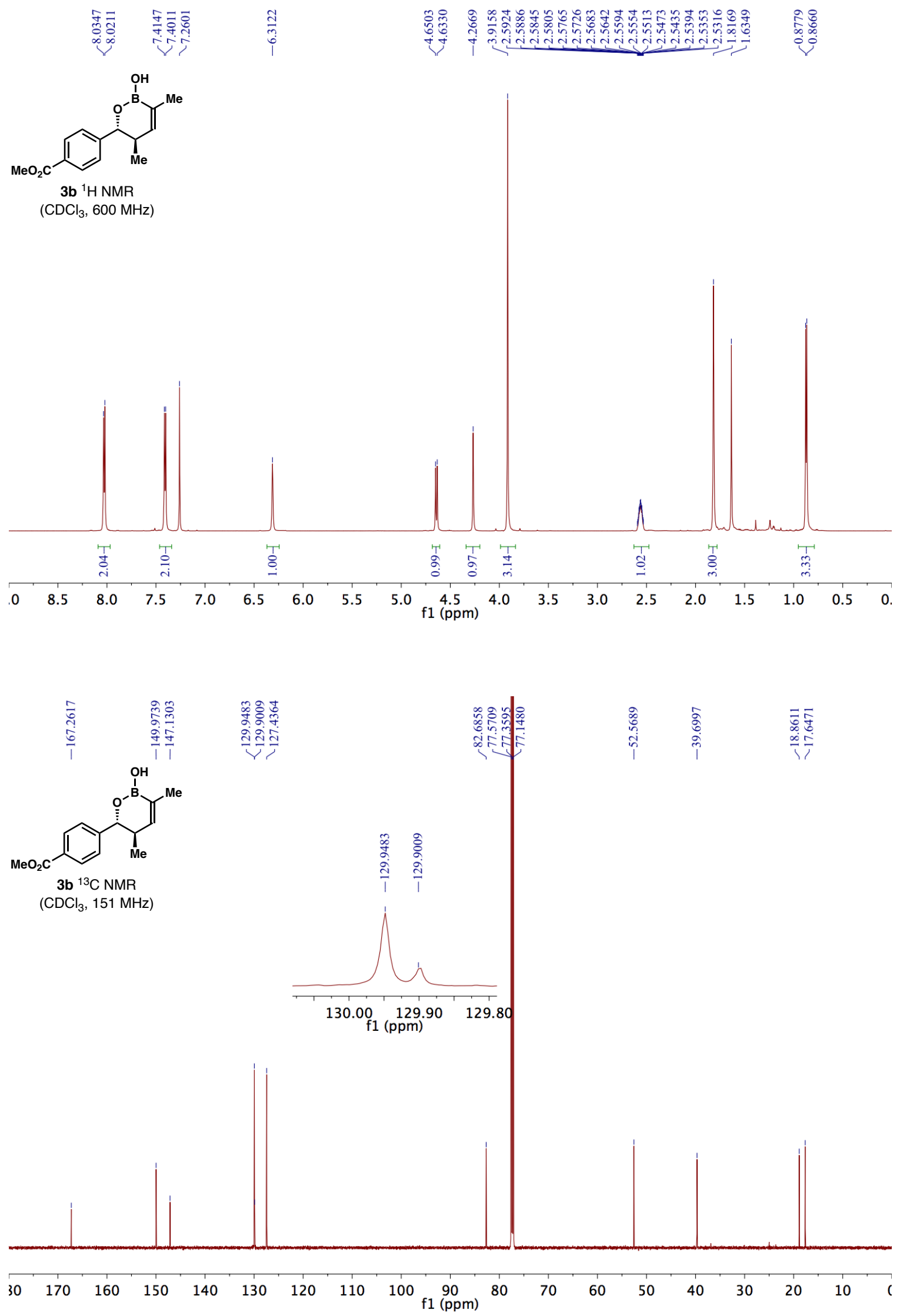

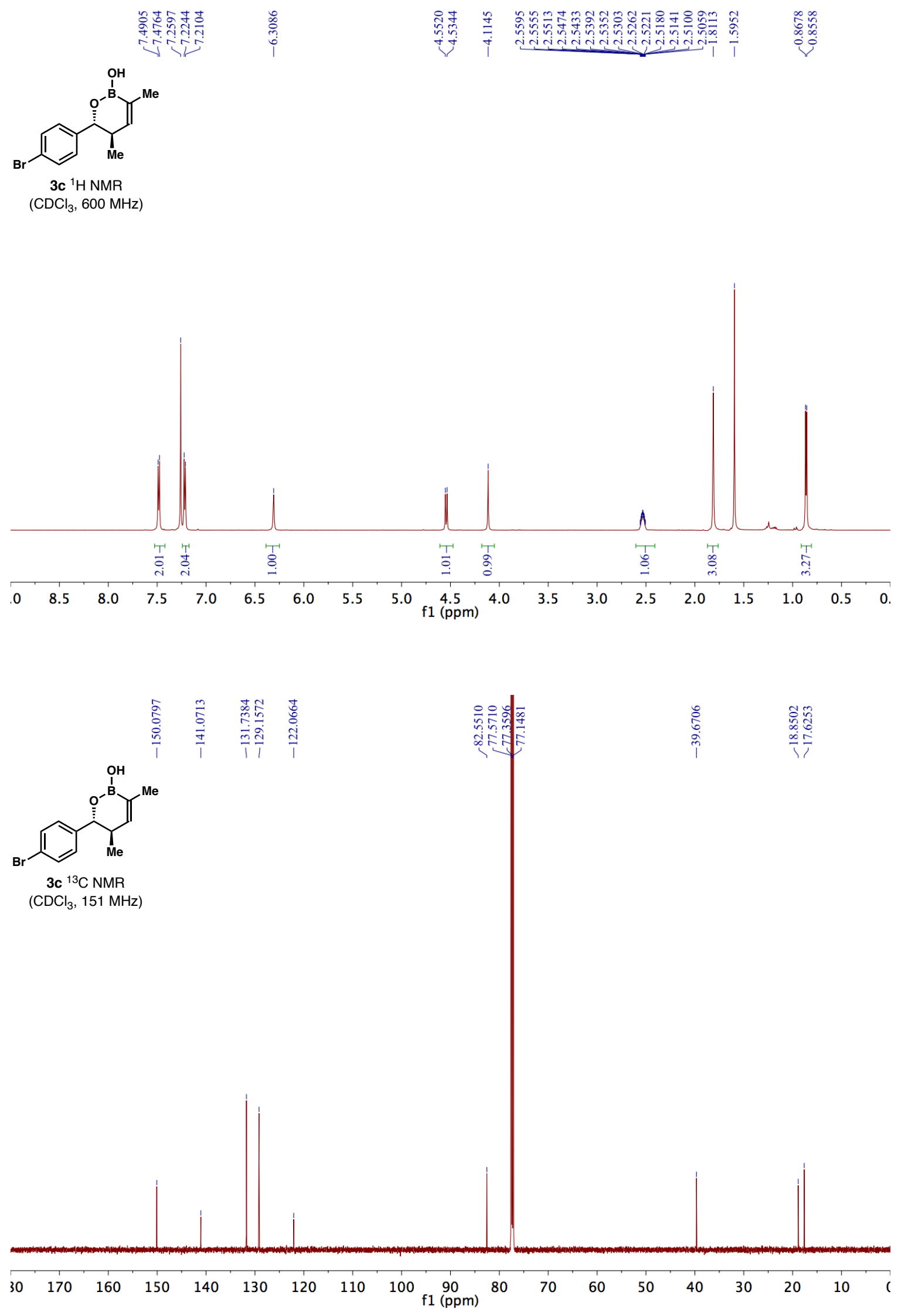

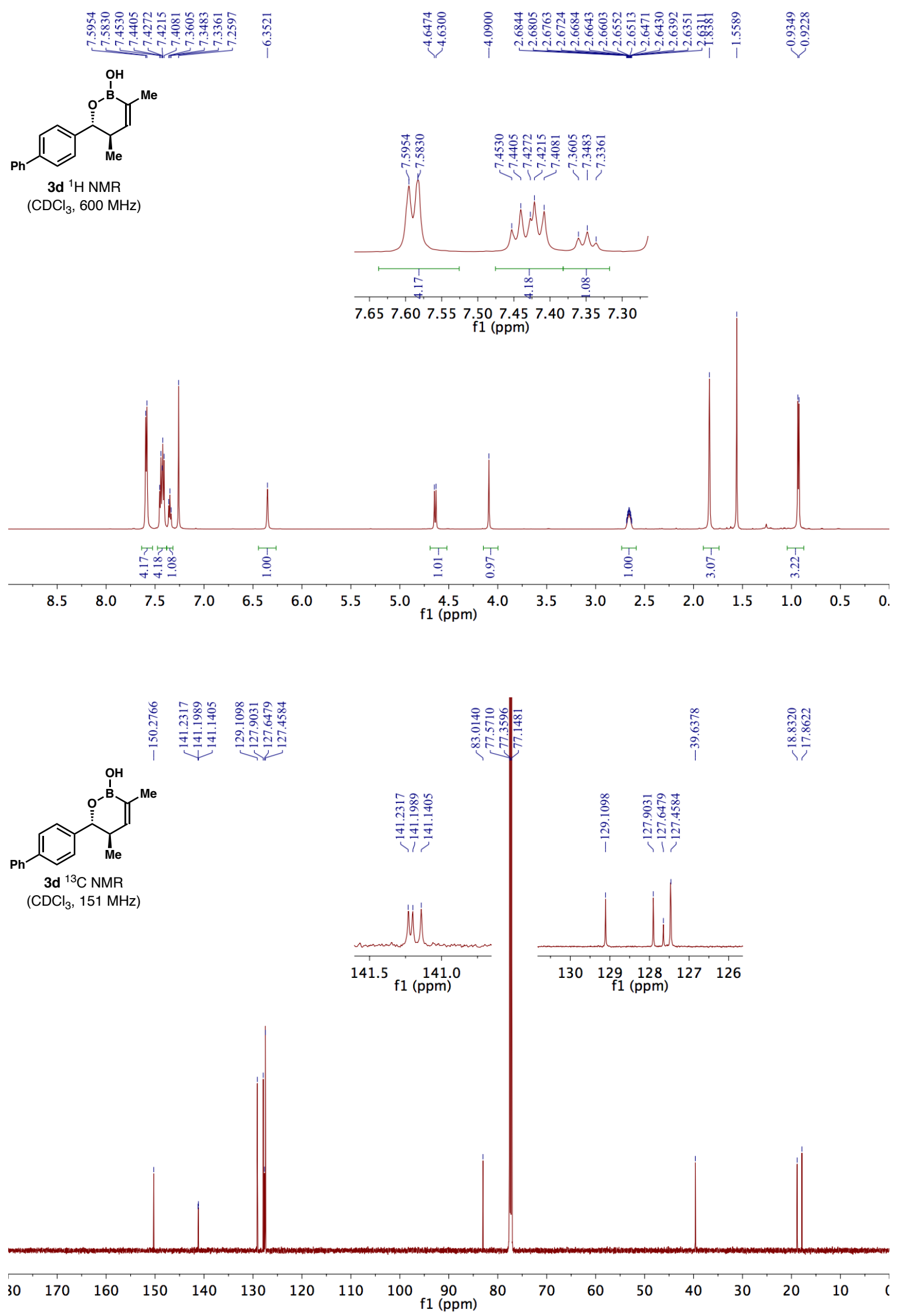

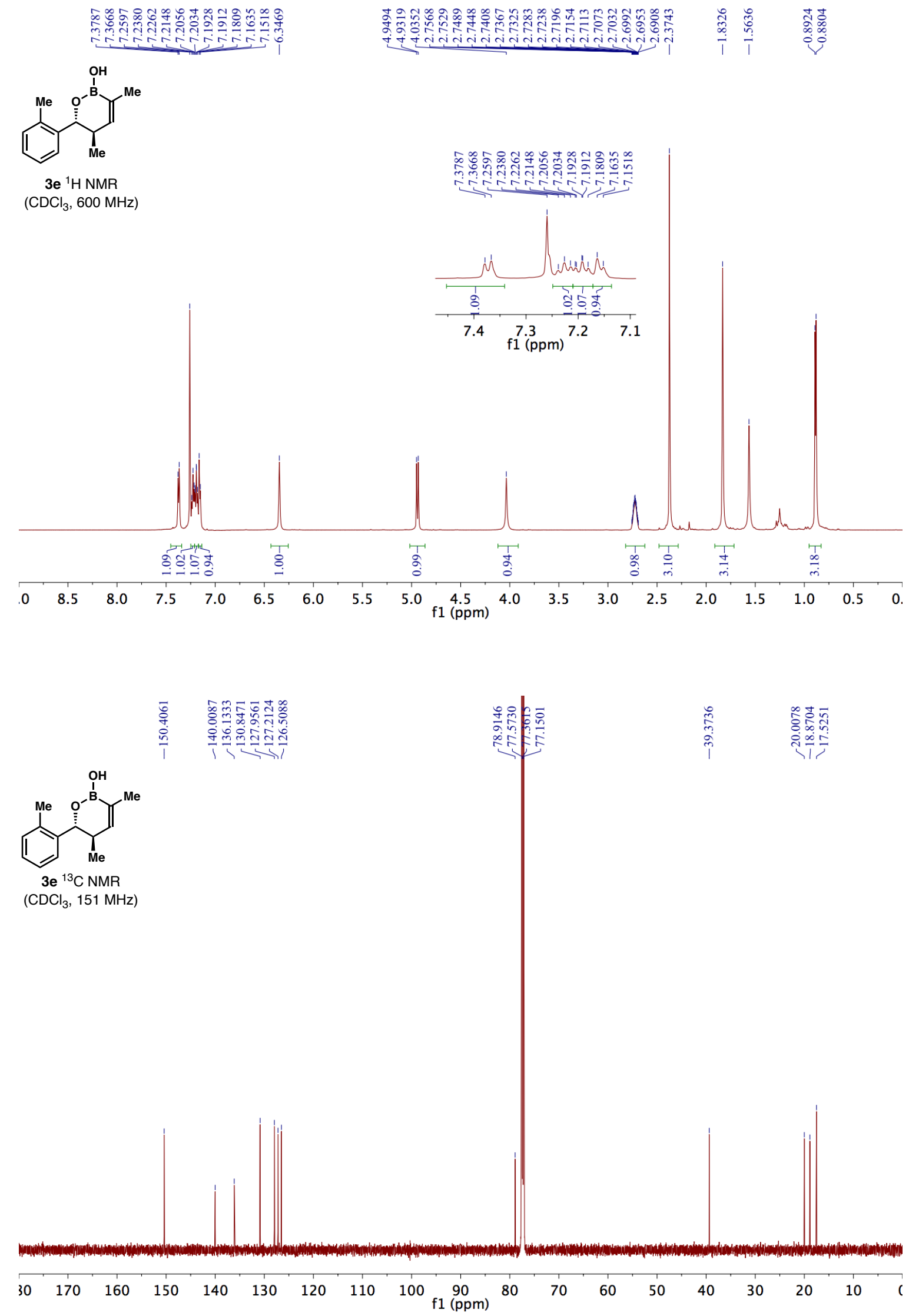

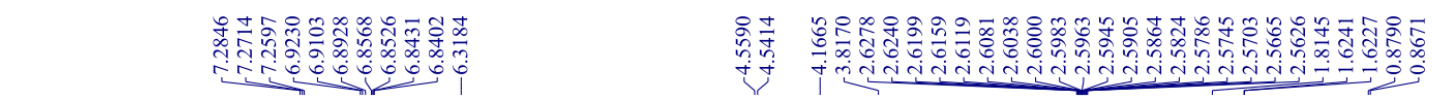

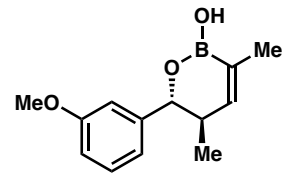

$3 f^{1} \mathrm{H}$ NMR

$\left(\mathrm{CDCl}_{3}, 600 \mathrm{MHz}\right)$
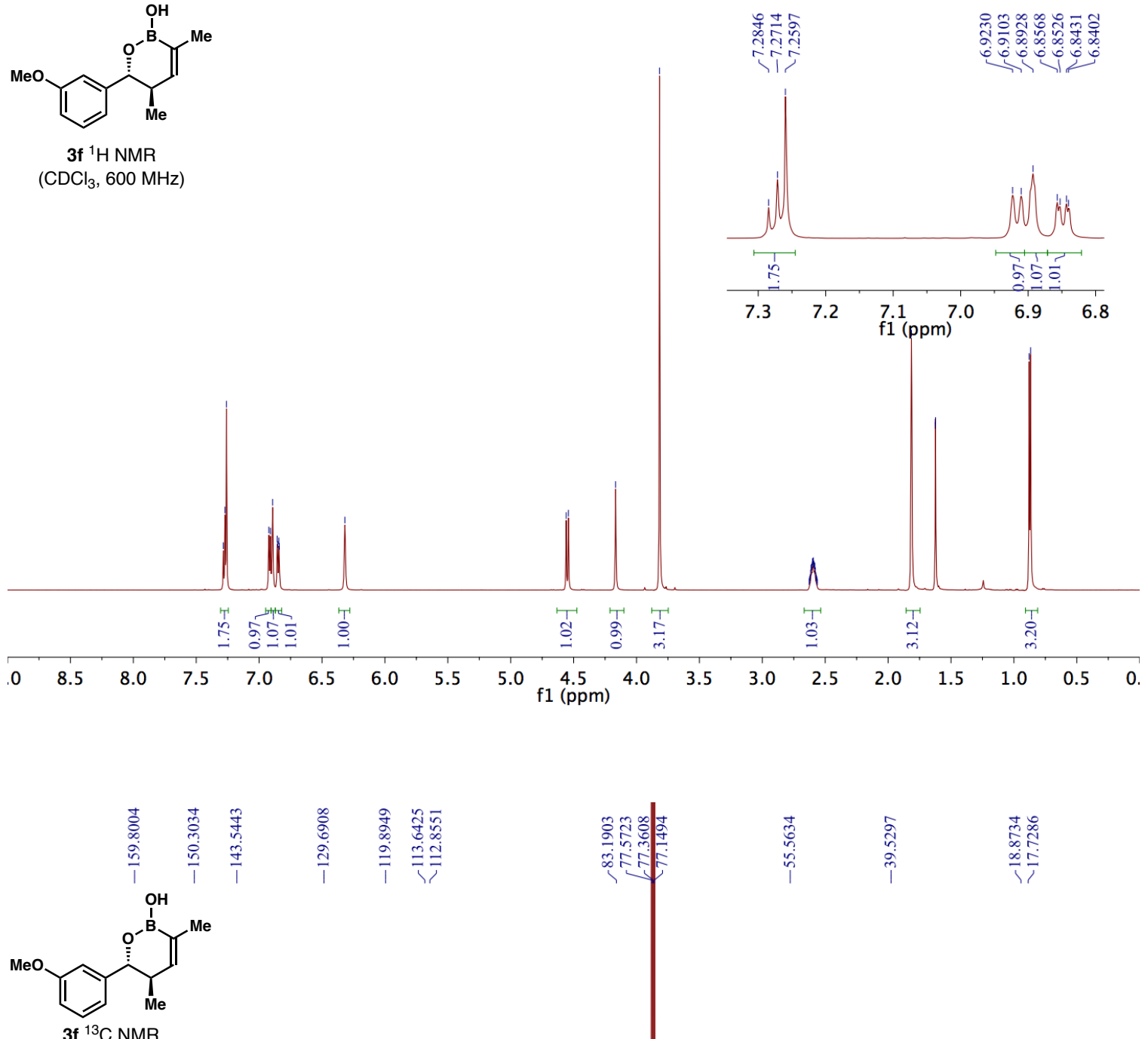

$3 \mathrm{H}^{13} \mathrm{C}$ NMR

$\left(\mathrm{CDCl}_{3}, 151 \mathrm{MHz}\right)$

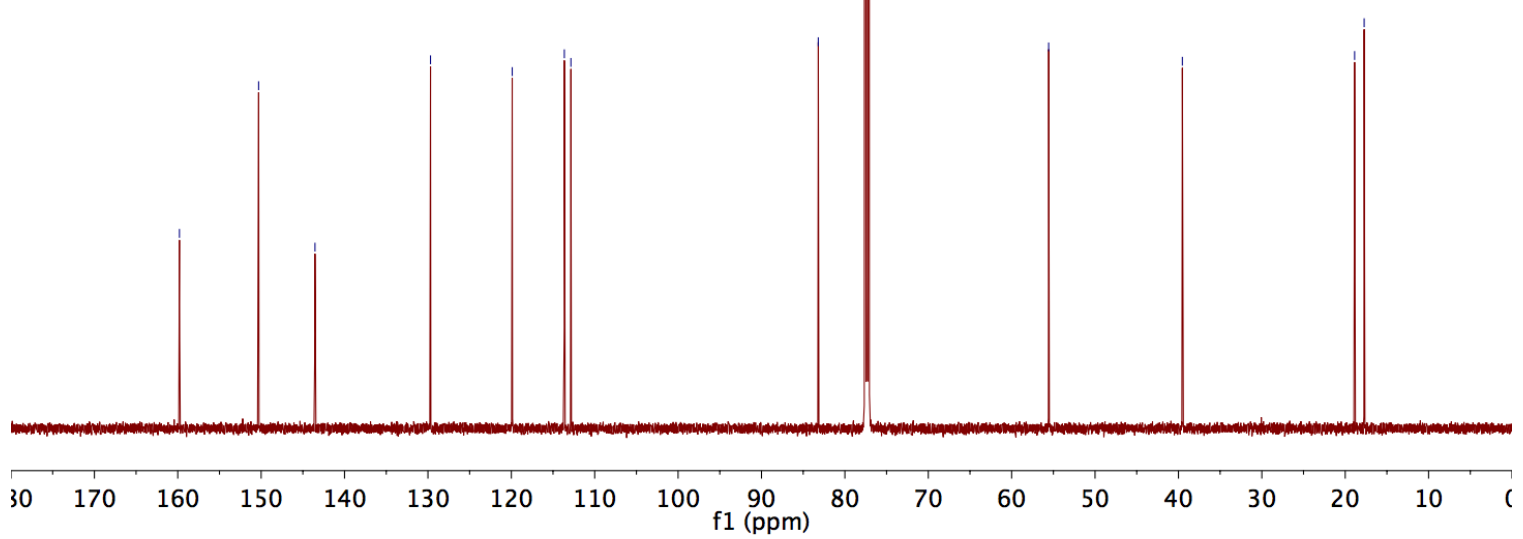



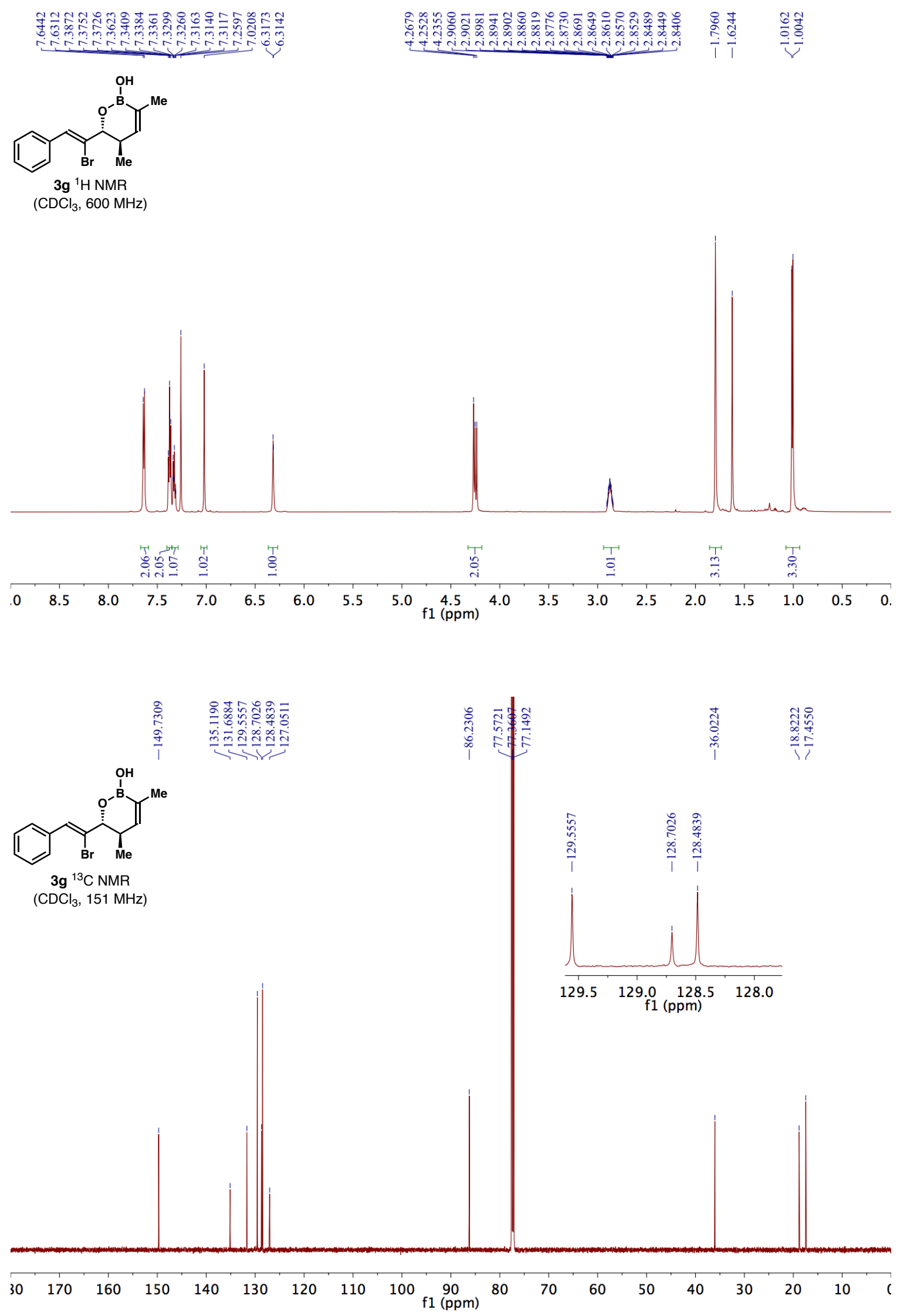

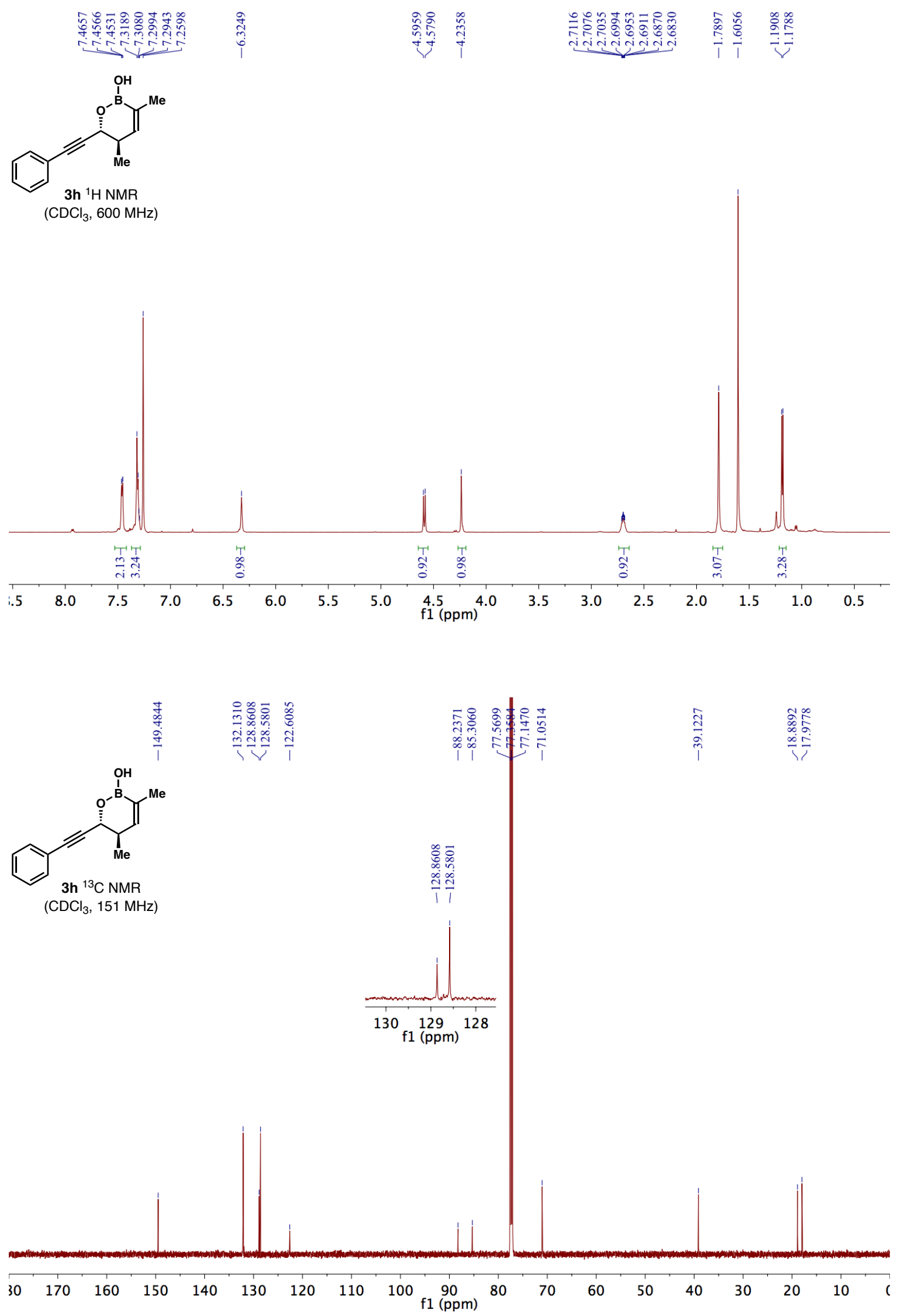

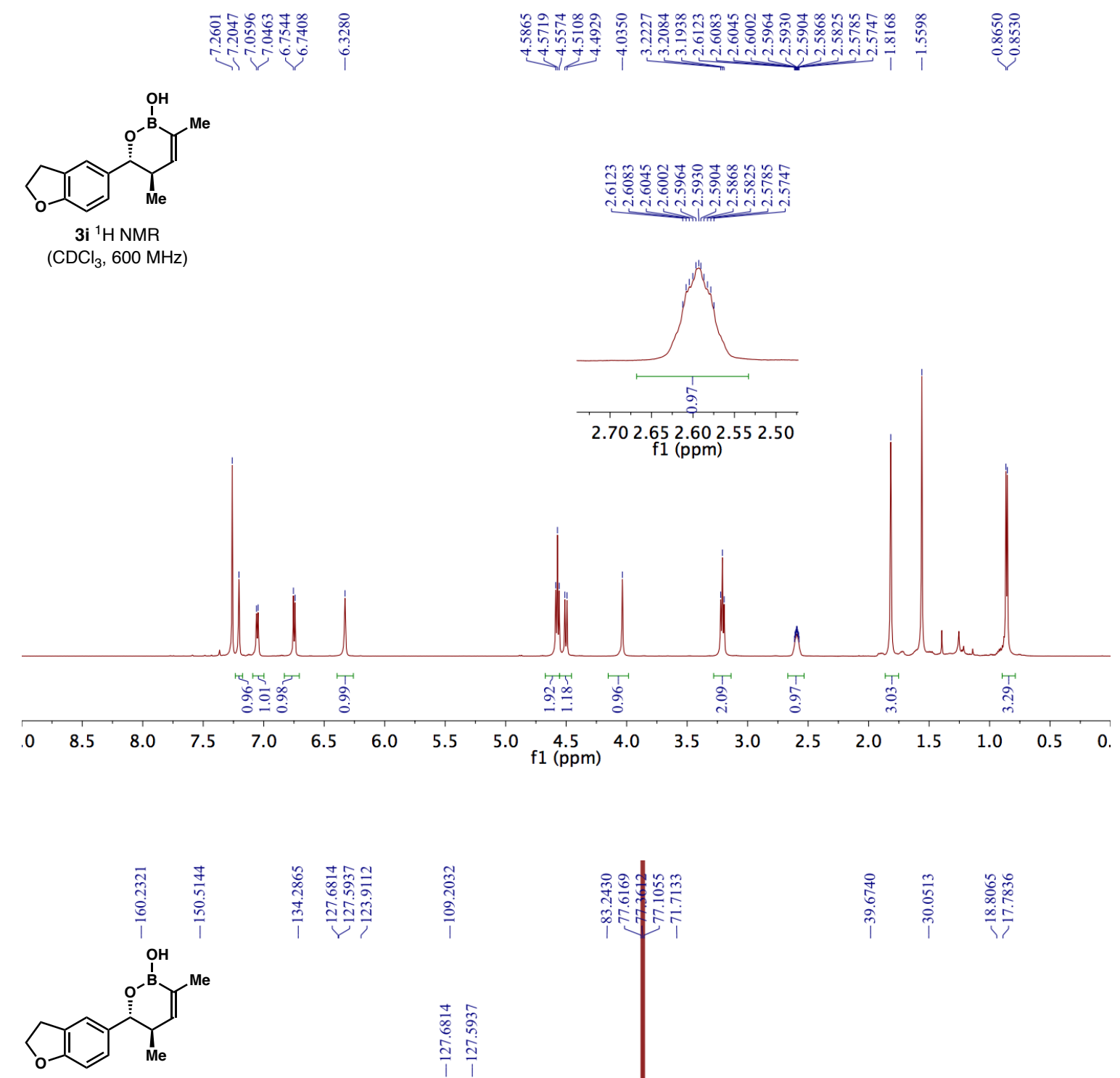

$3 \mathbf{~}^{13} \mathrm{C}$ NMR

$\left(\mathrm{CDCl}_{3}, 126 \mathrm{MHz}\right)$
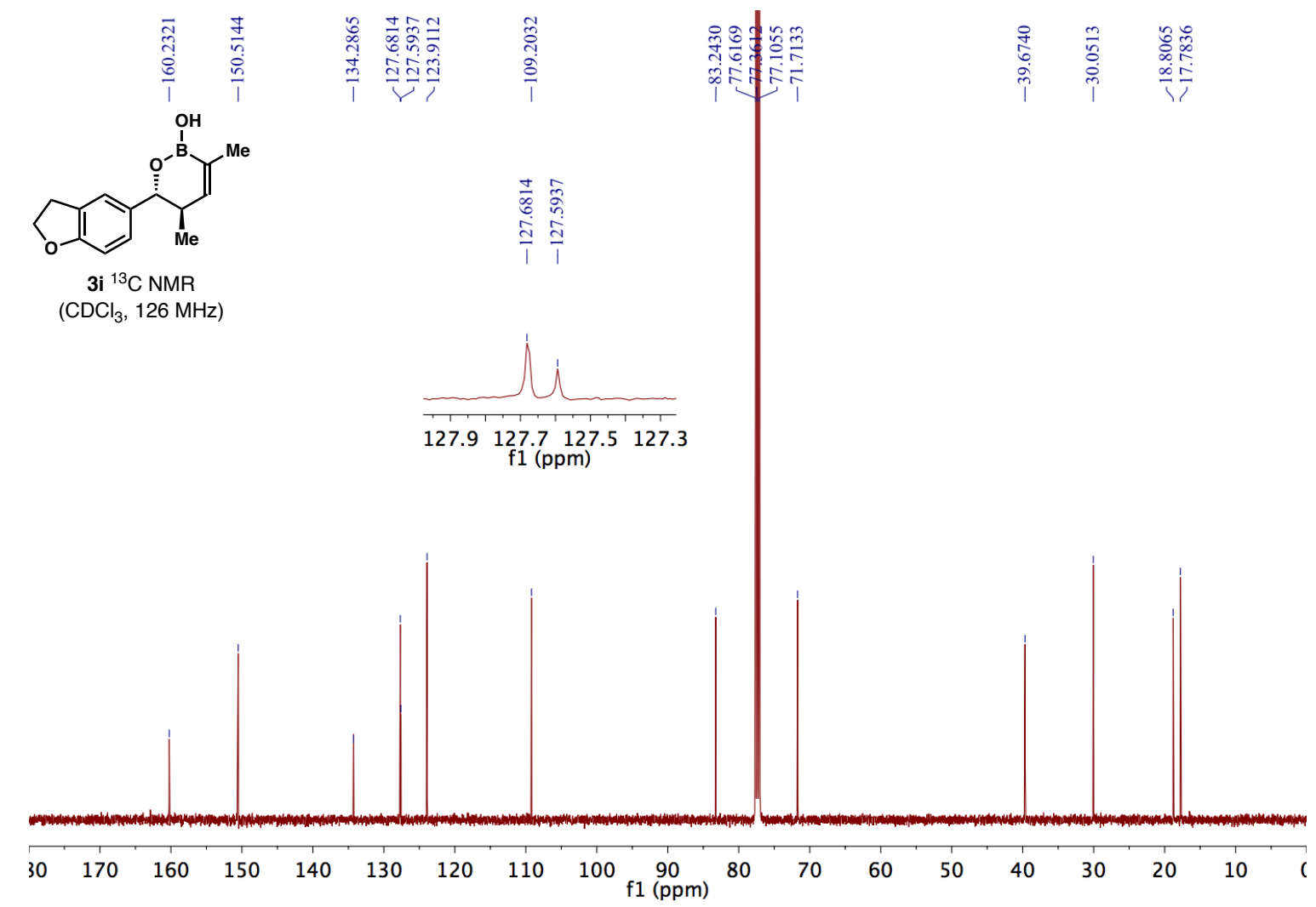

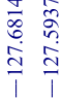

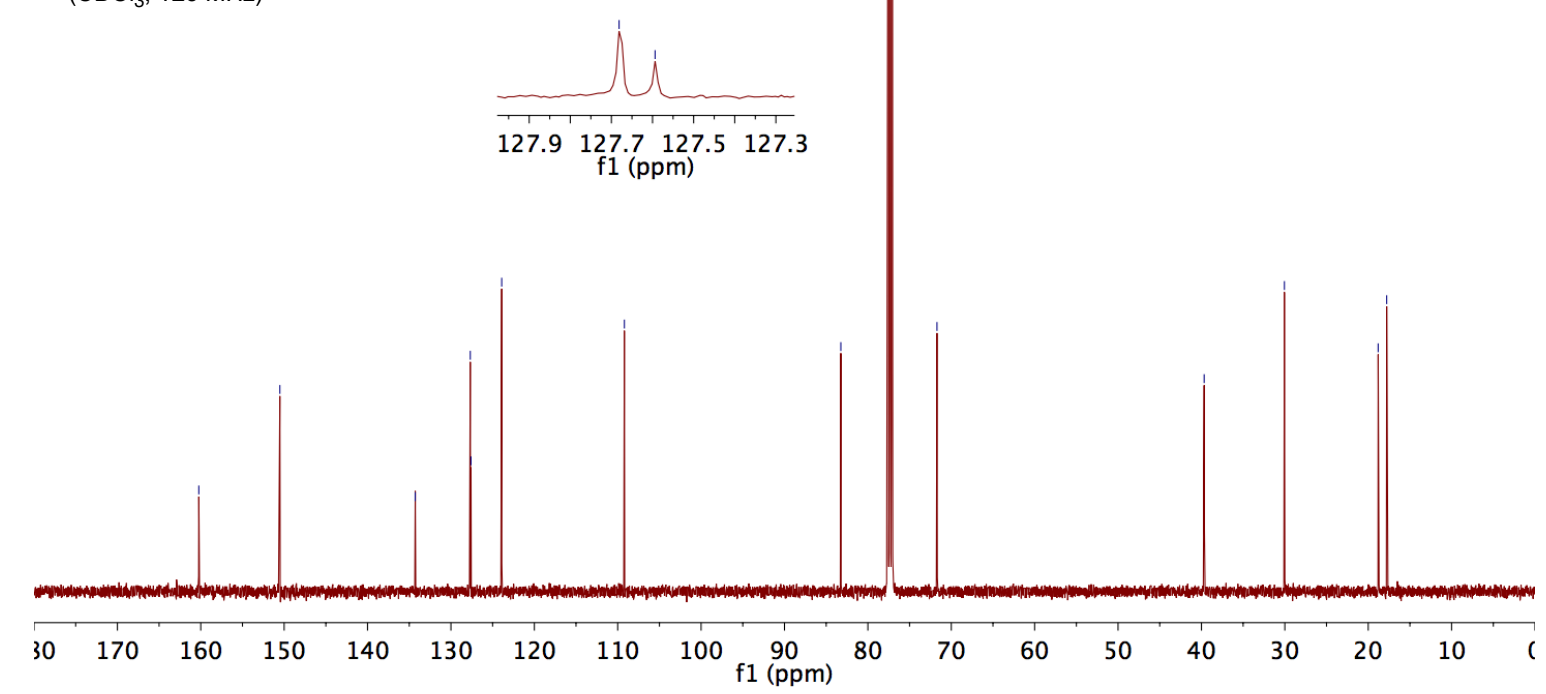



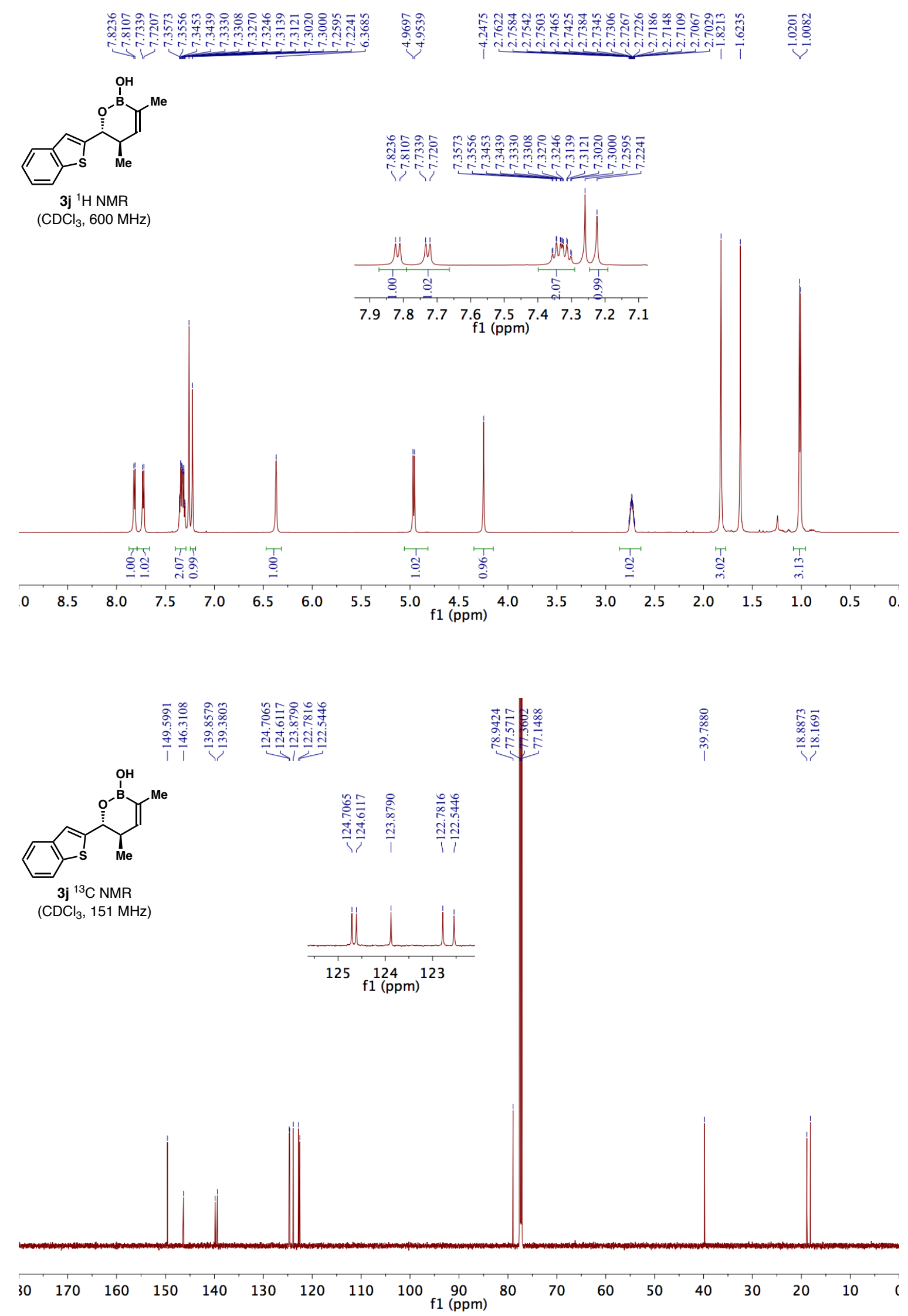

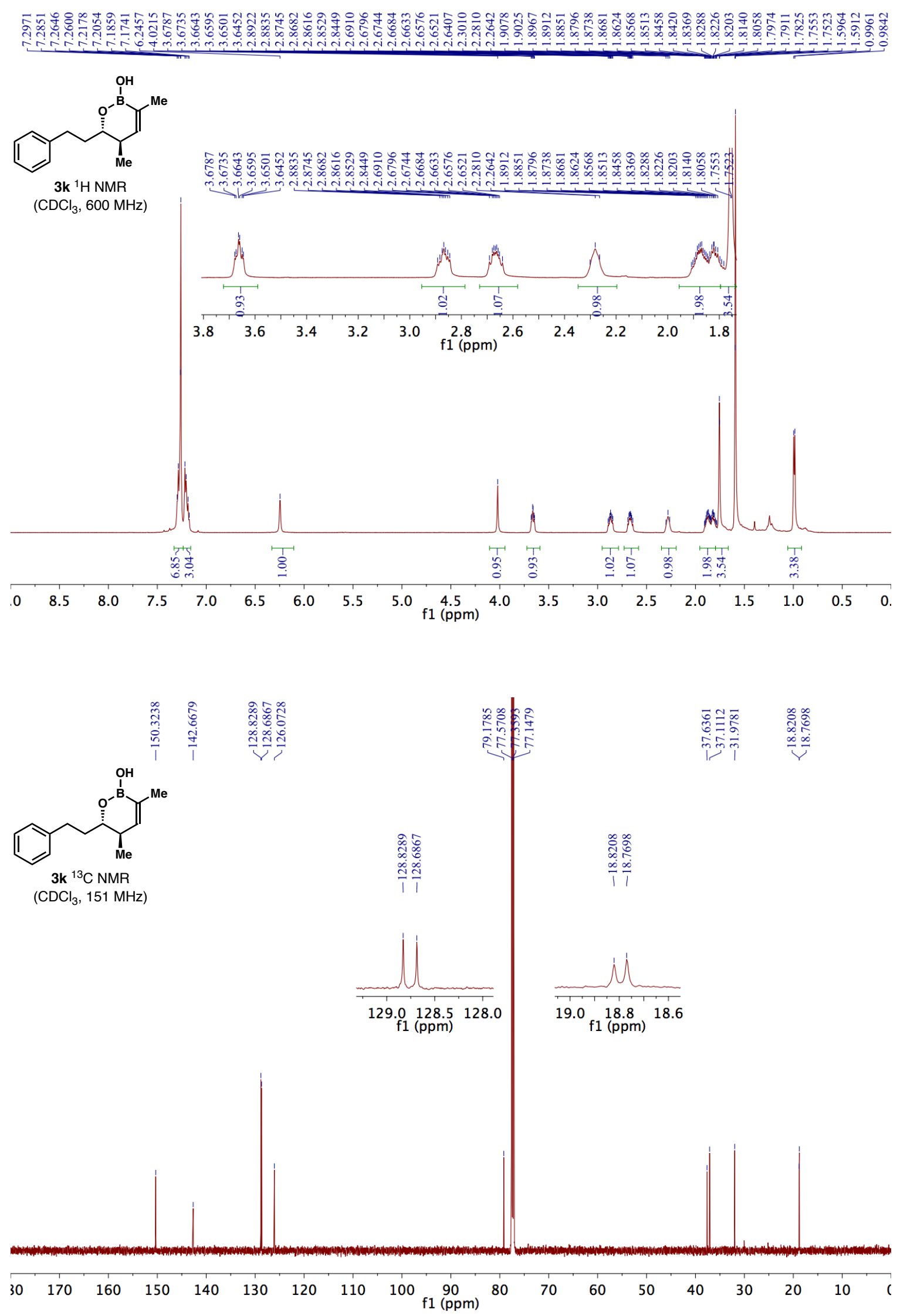


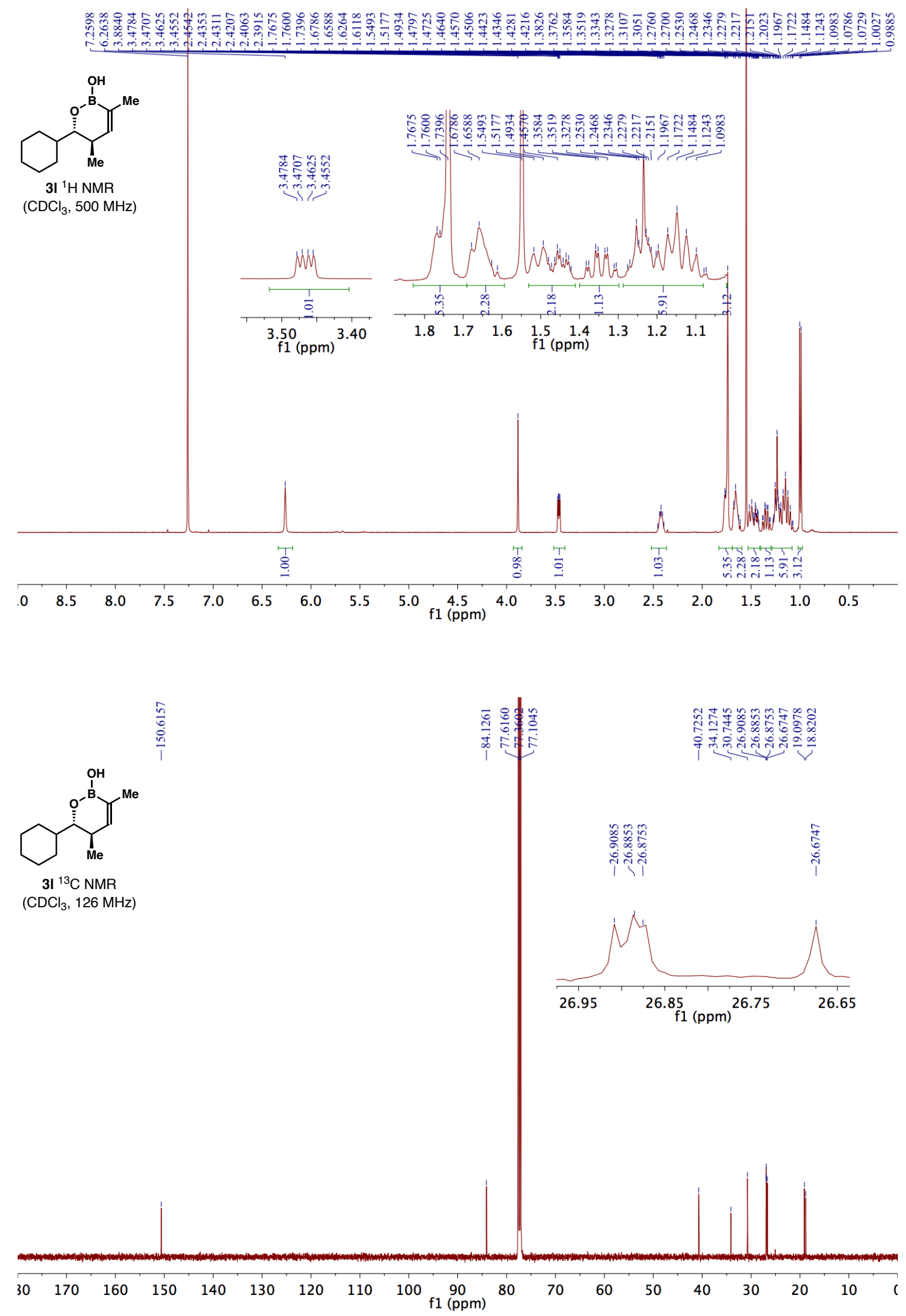



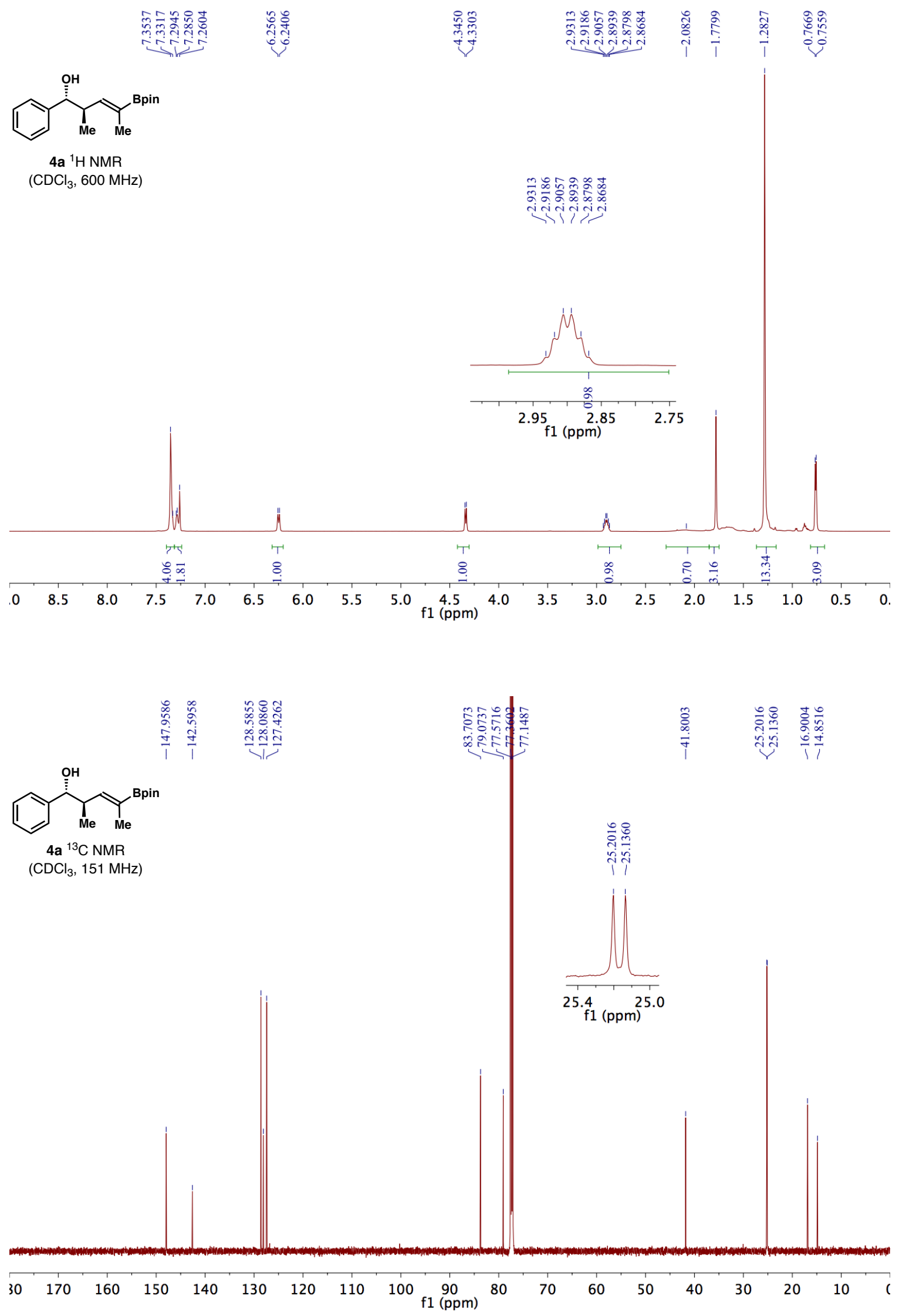

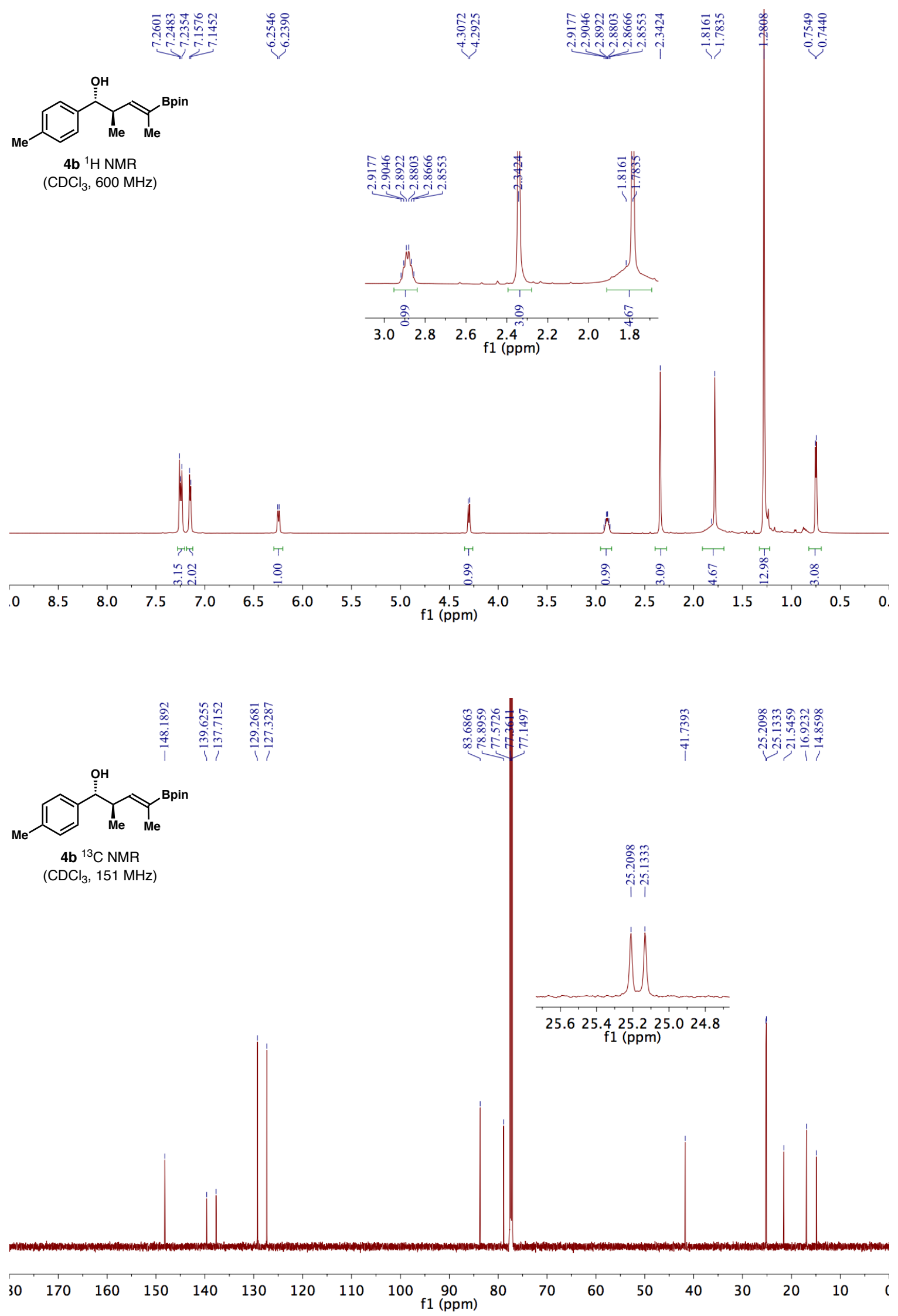

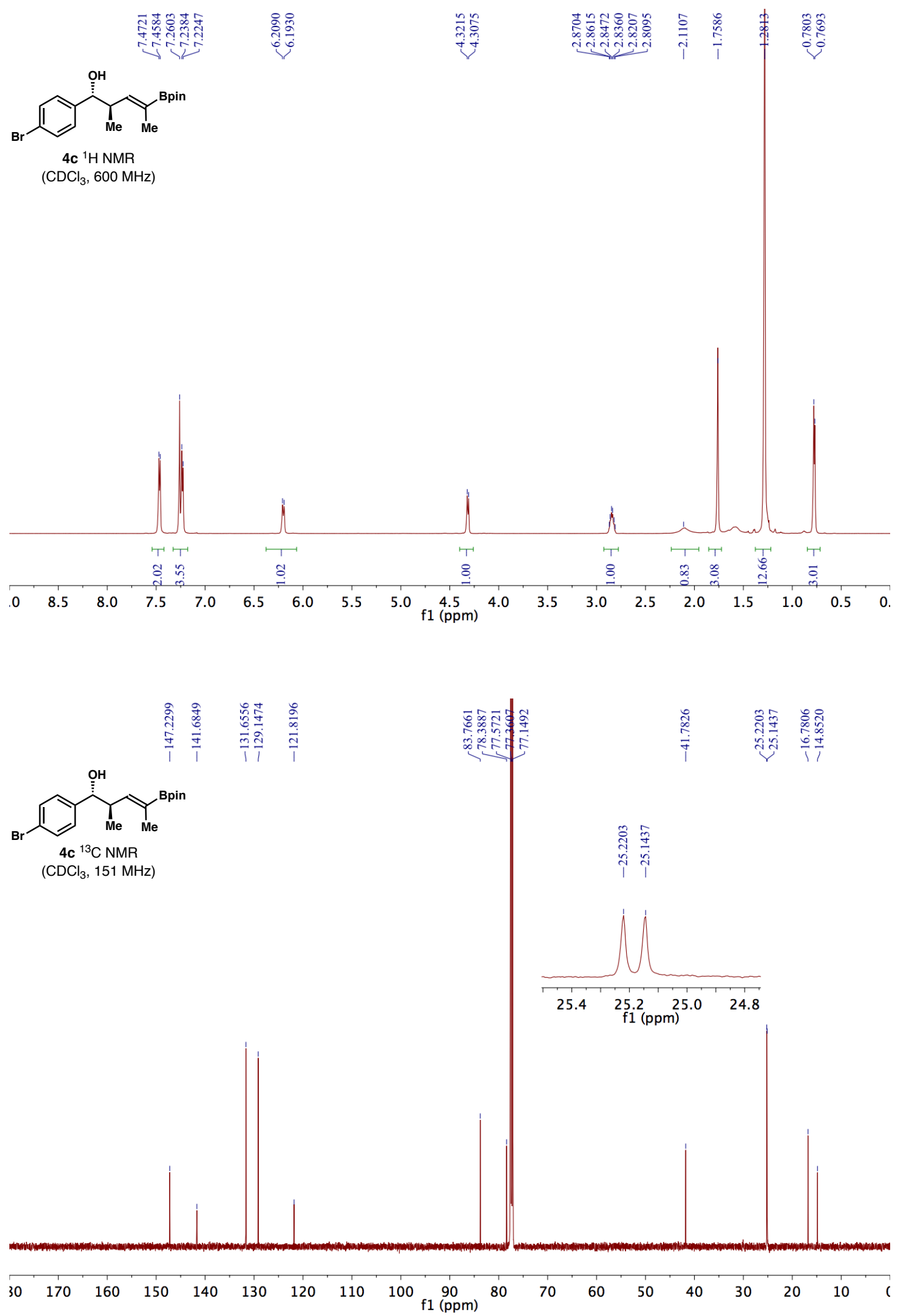

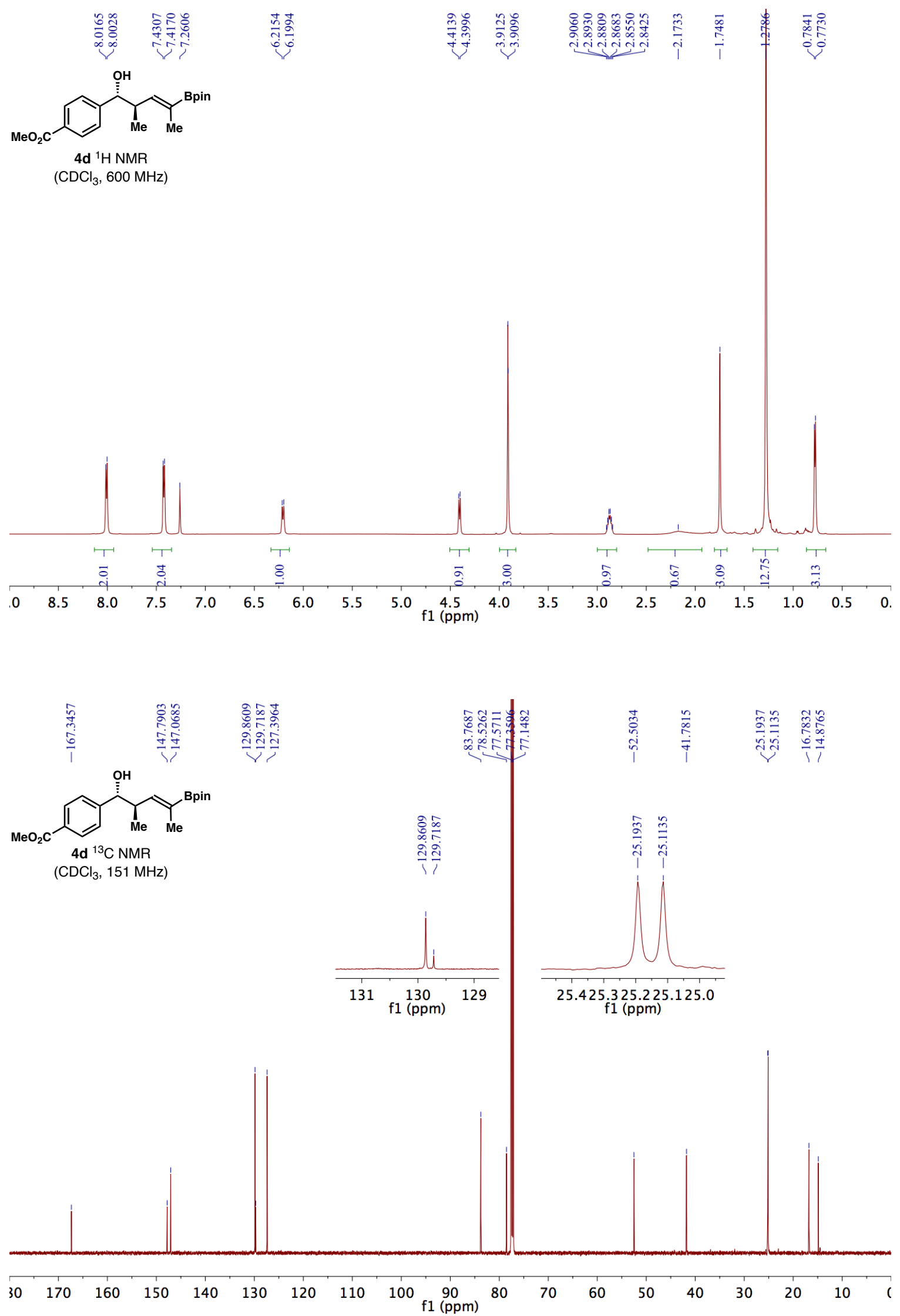

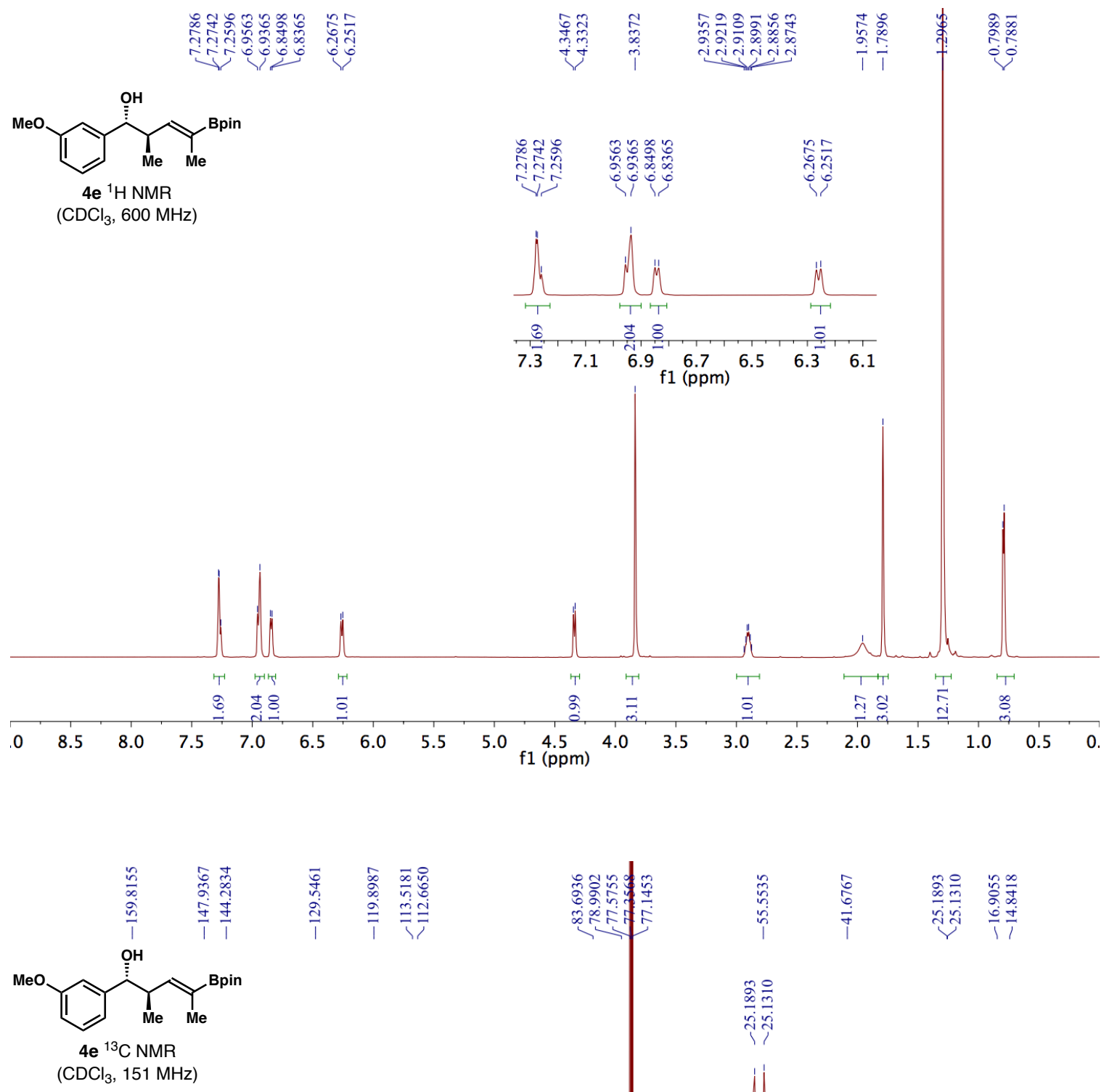

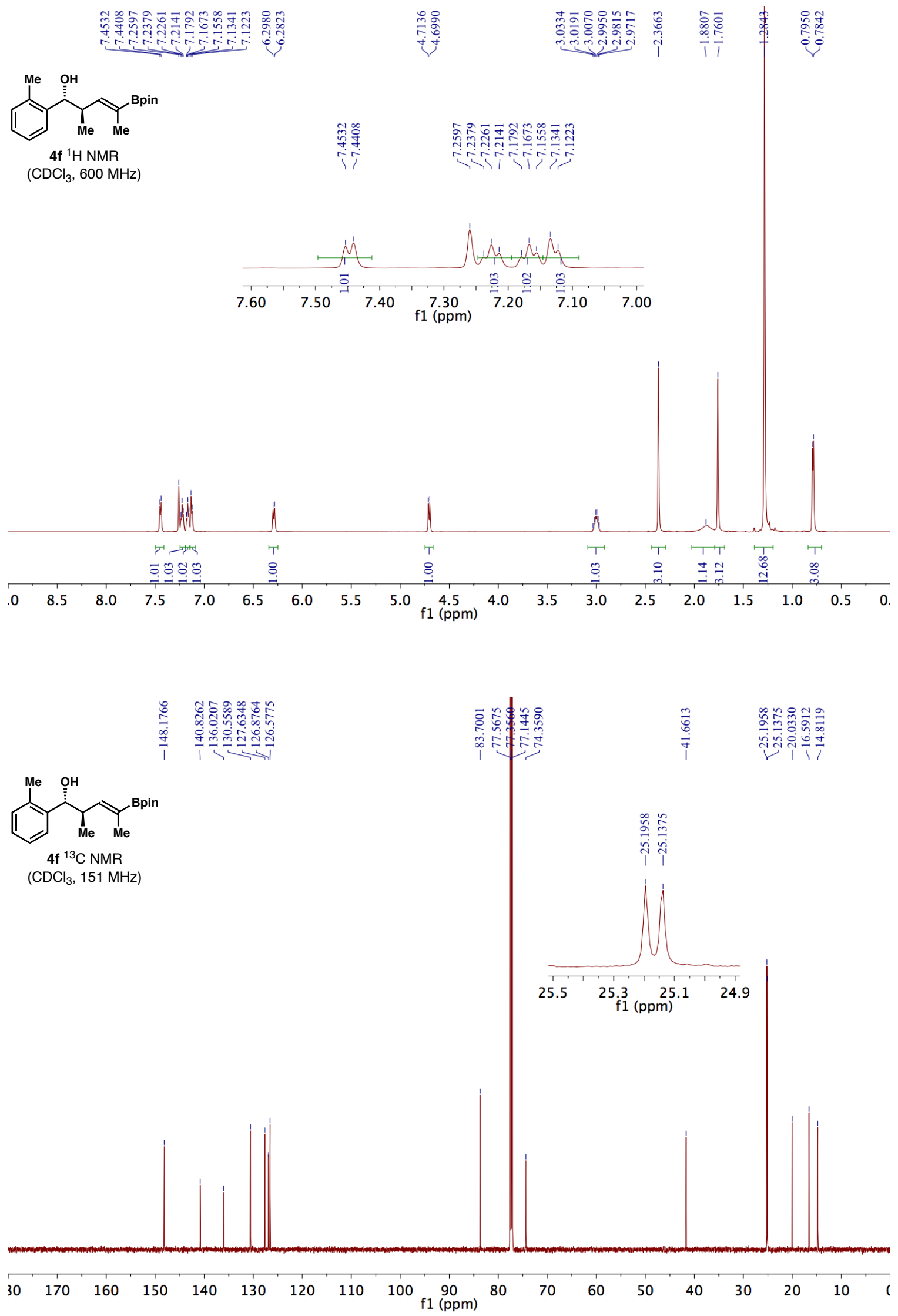

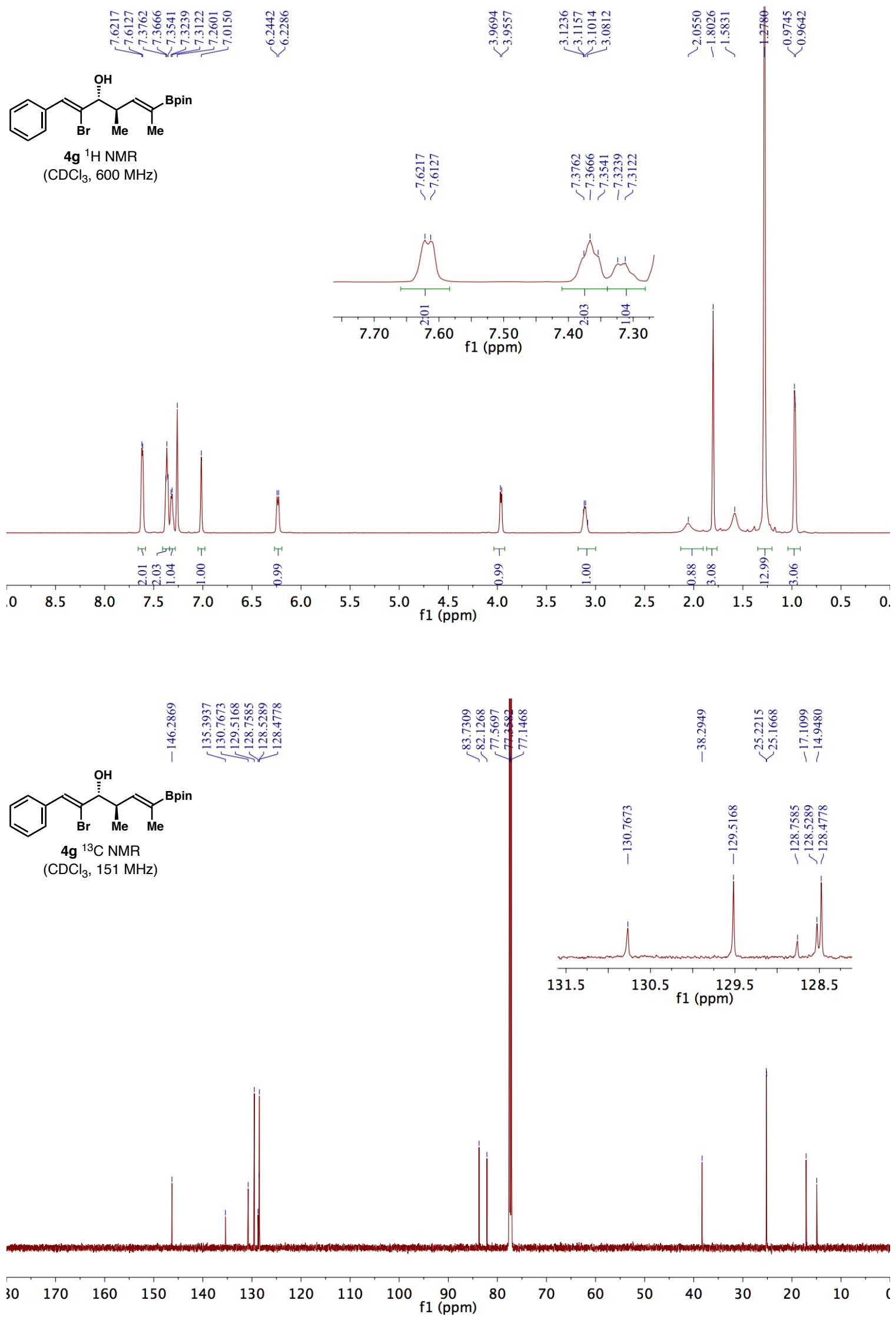

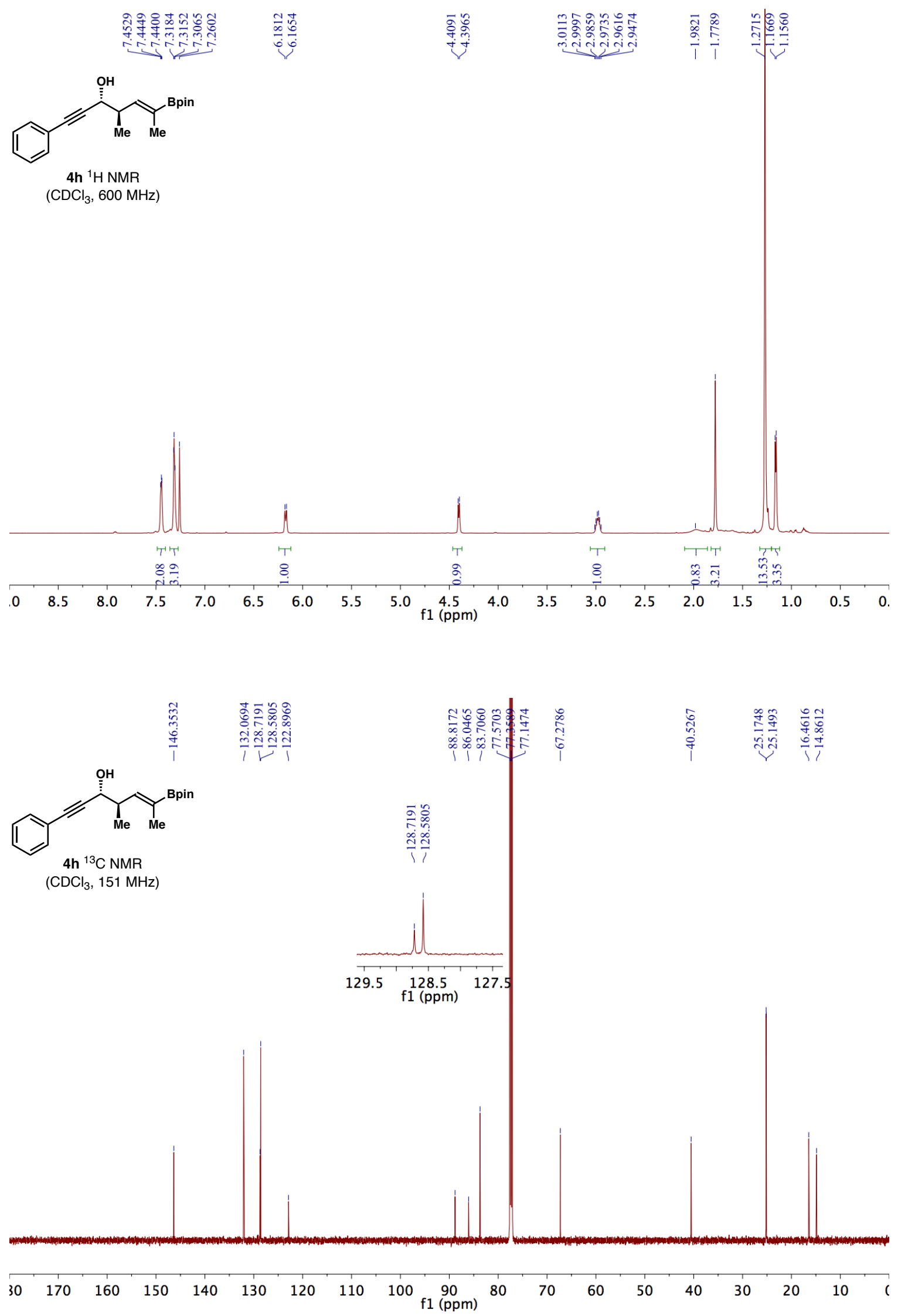

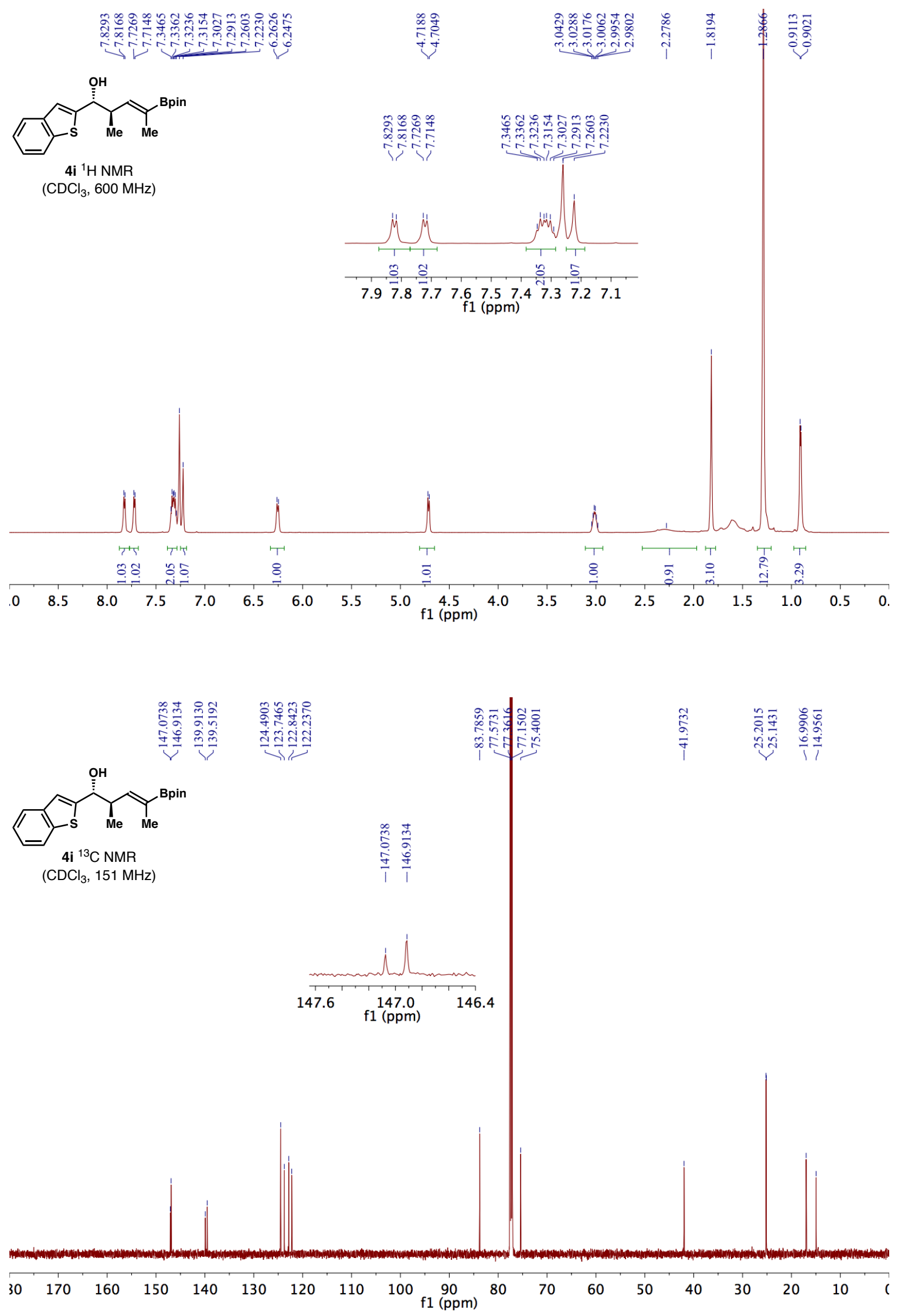

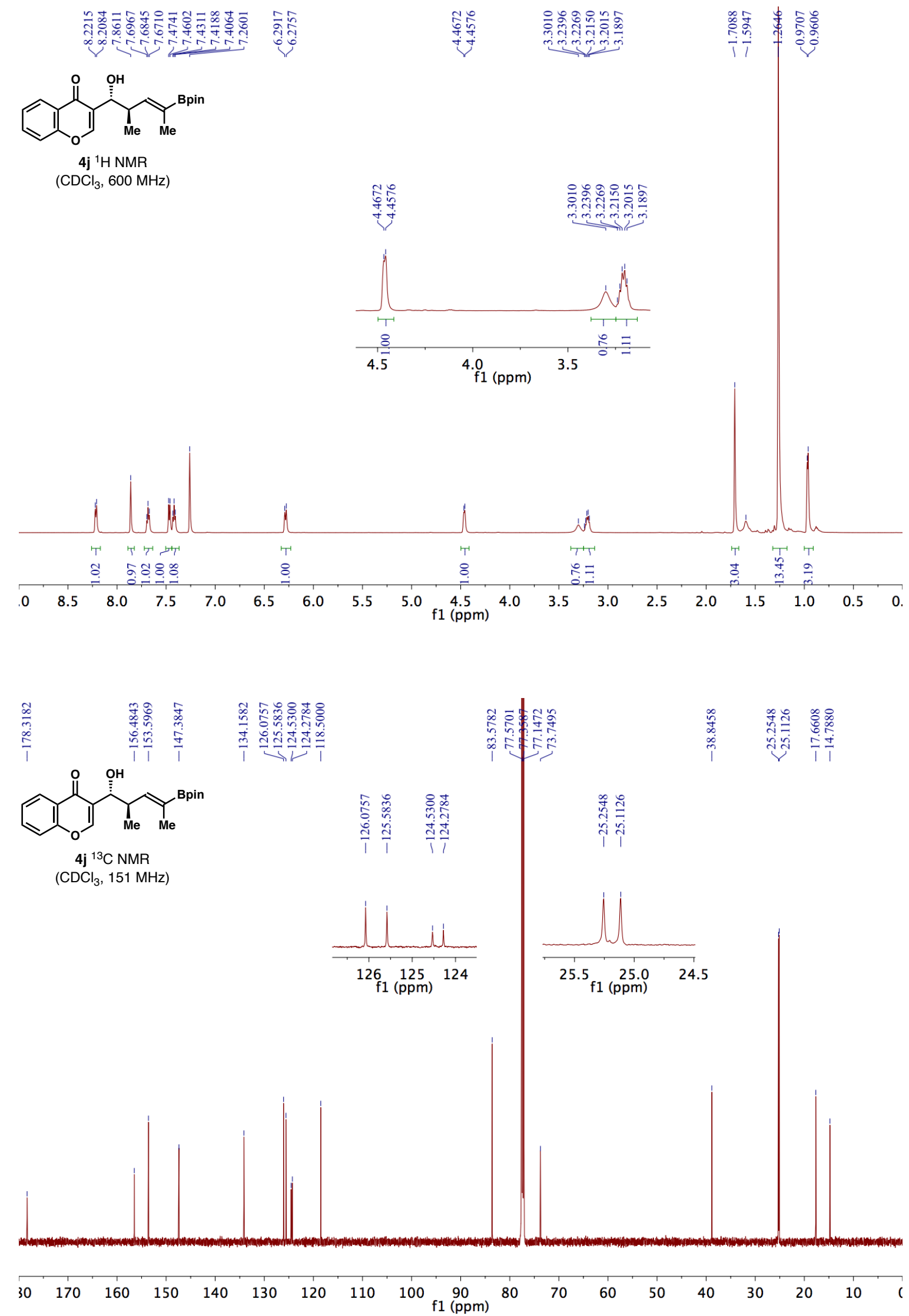

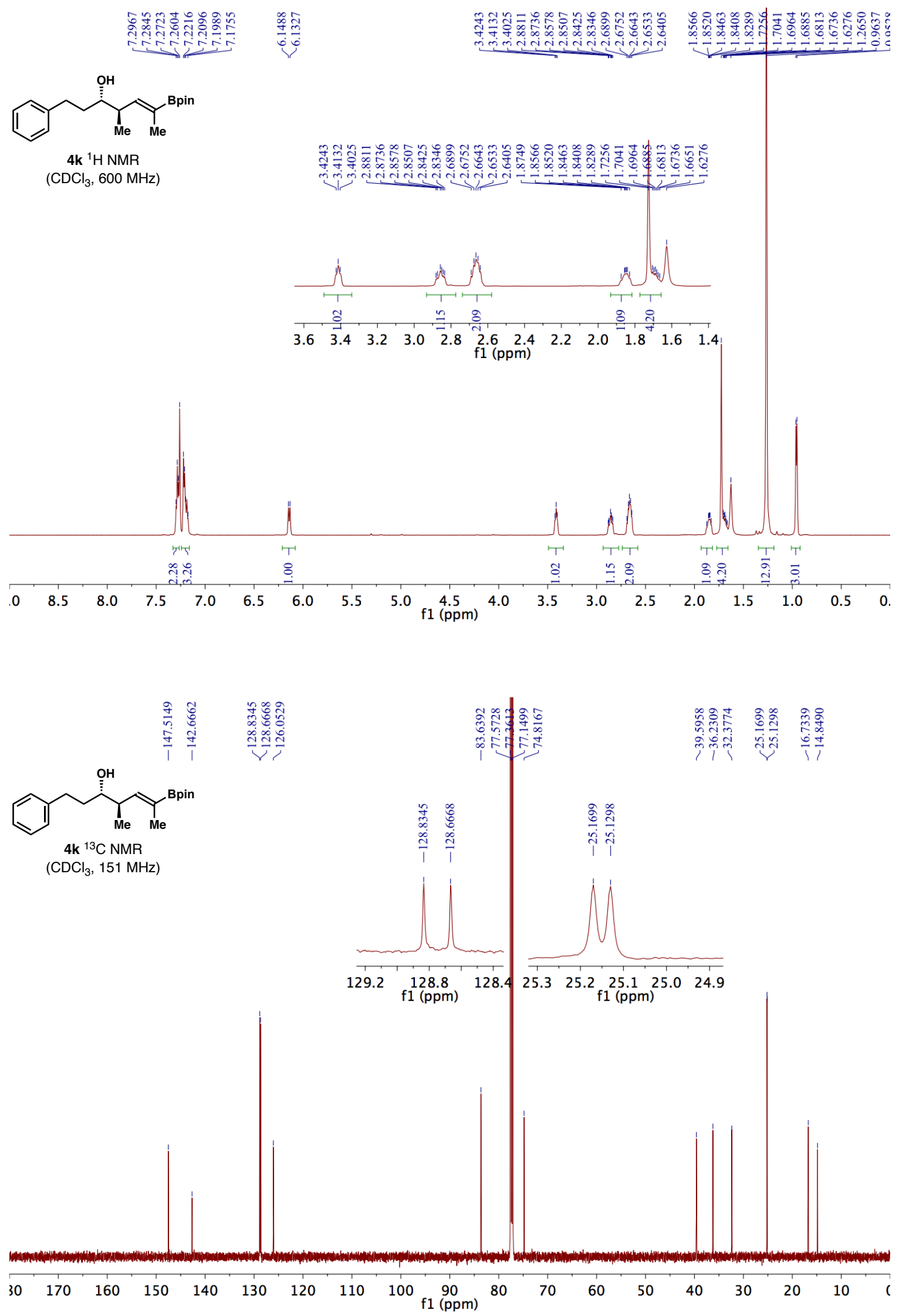

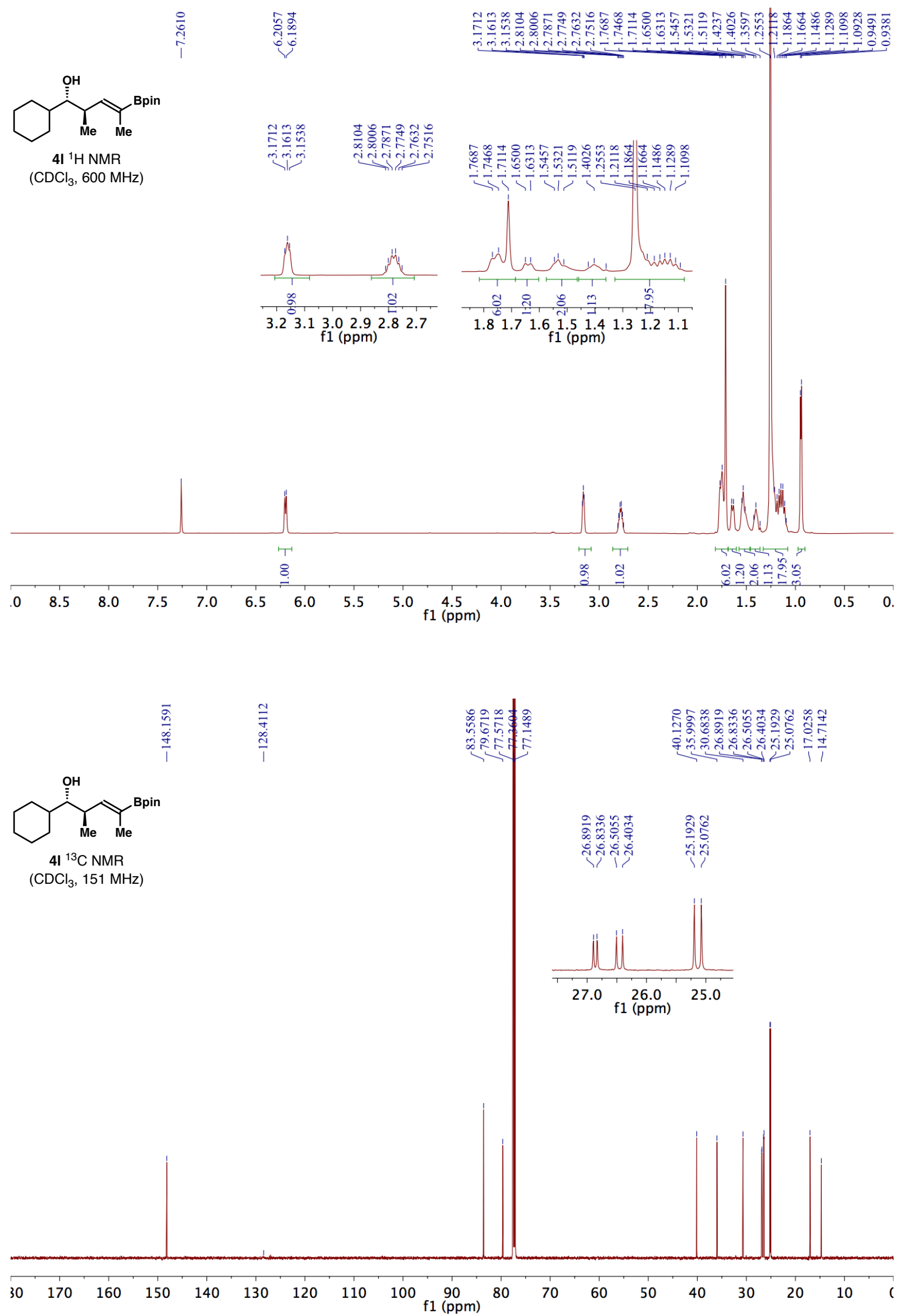

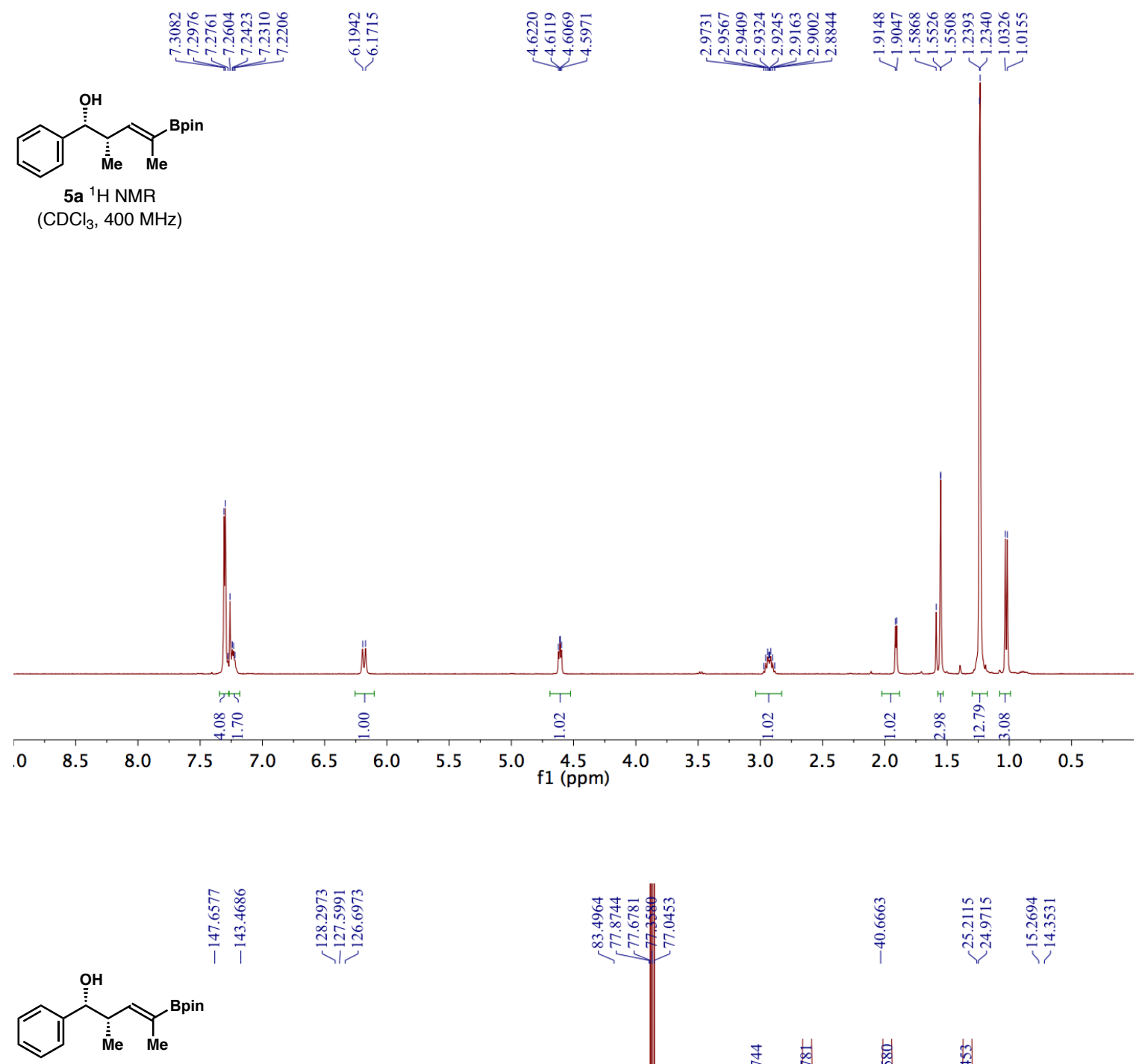

$5{ }^{13} \mathrm{C}$ NMR

$\left(\mathrm{CDCl}_{3}, 101 \mathrm{MHz}\right)$

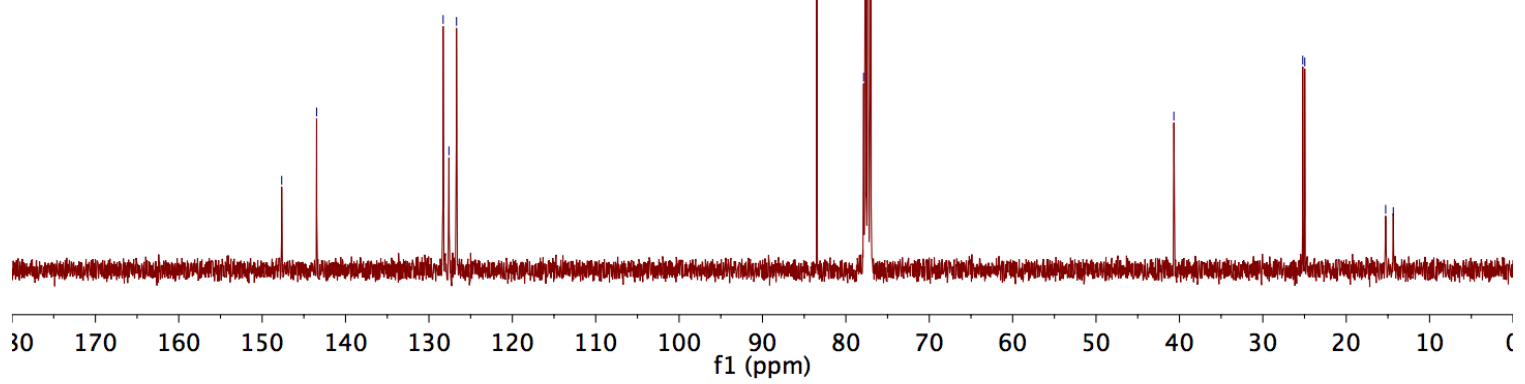



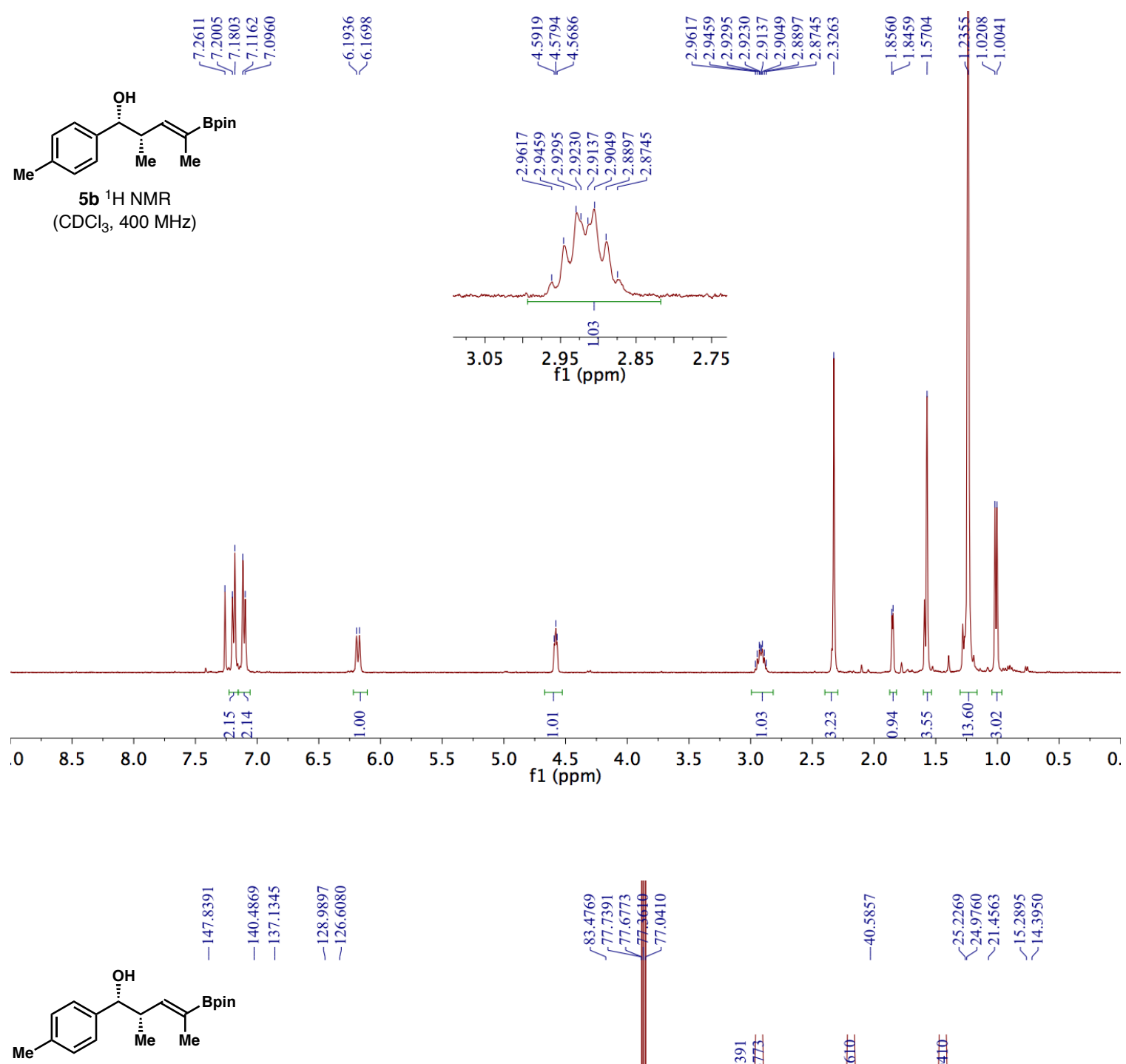

$5 b^{13} \mathrm{C}$ NMR

$\left(\mathrm{CDCl}_{3}, 101 \mathrm{MHz}\right)$

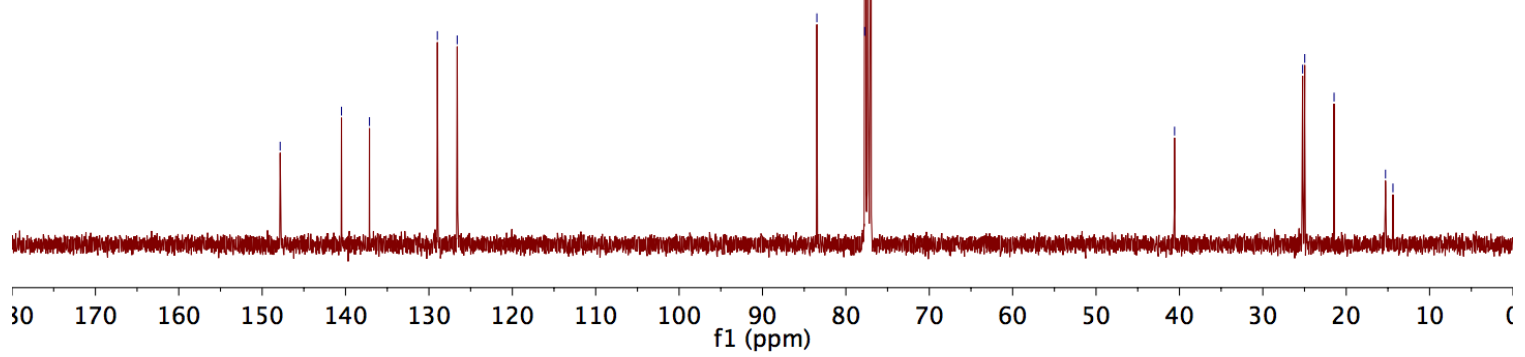



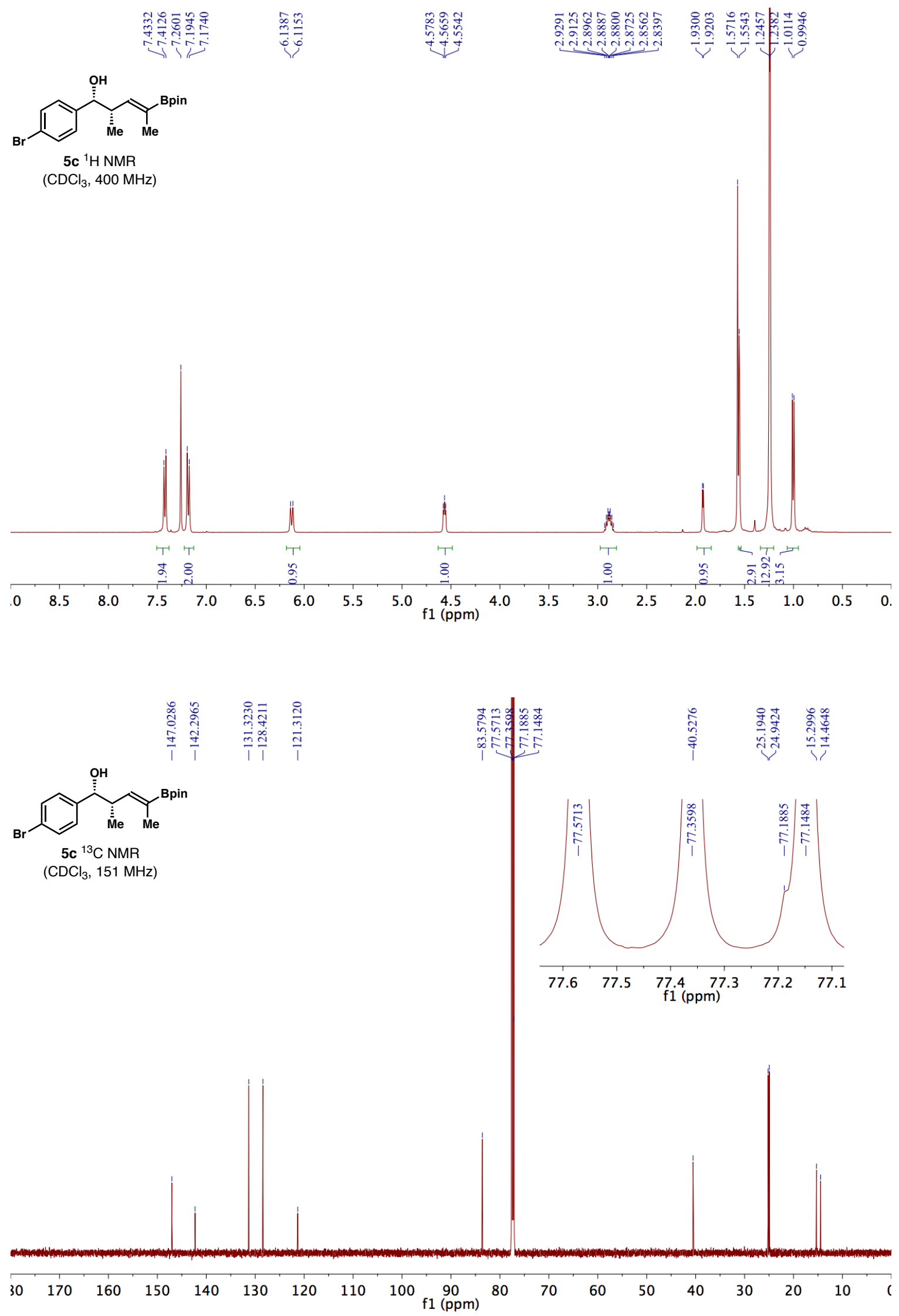

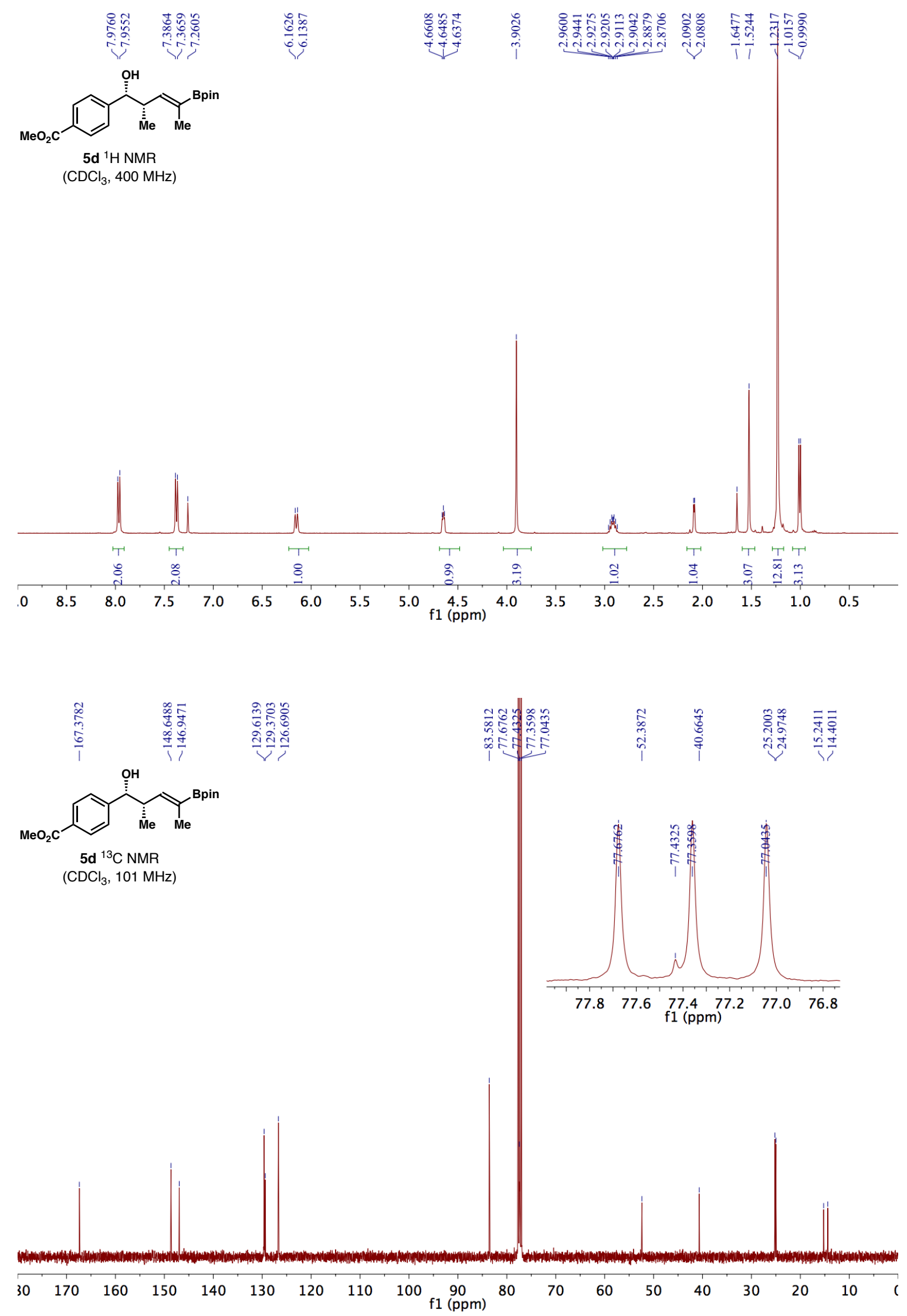

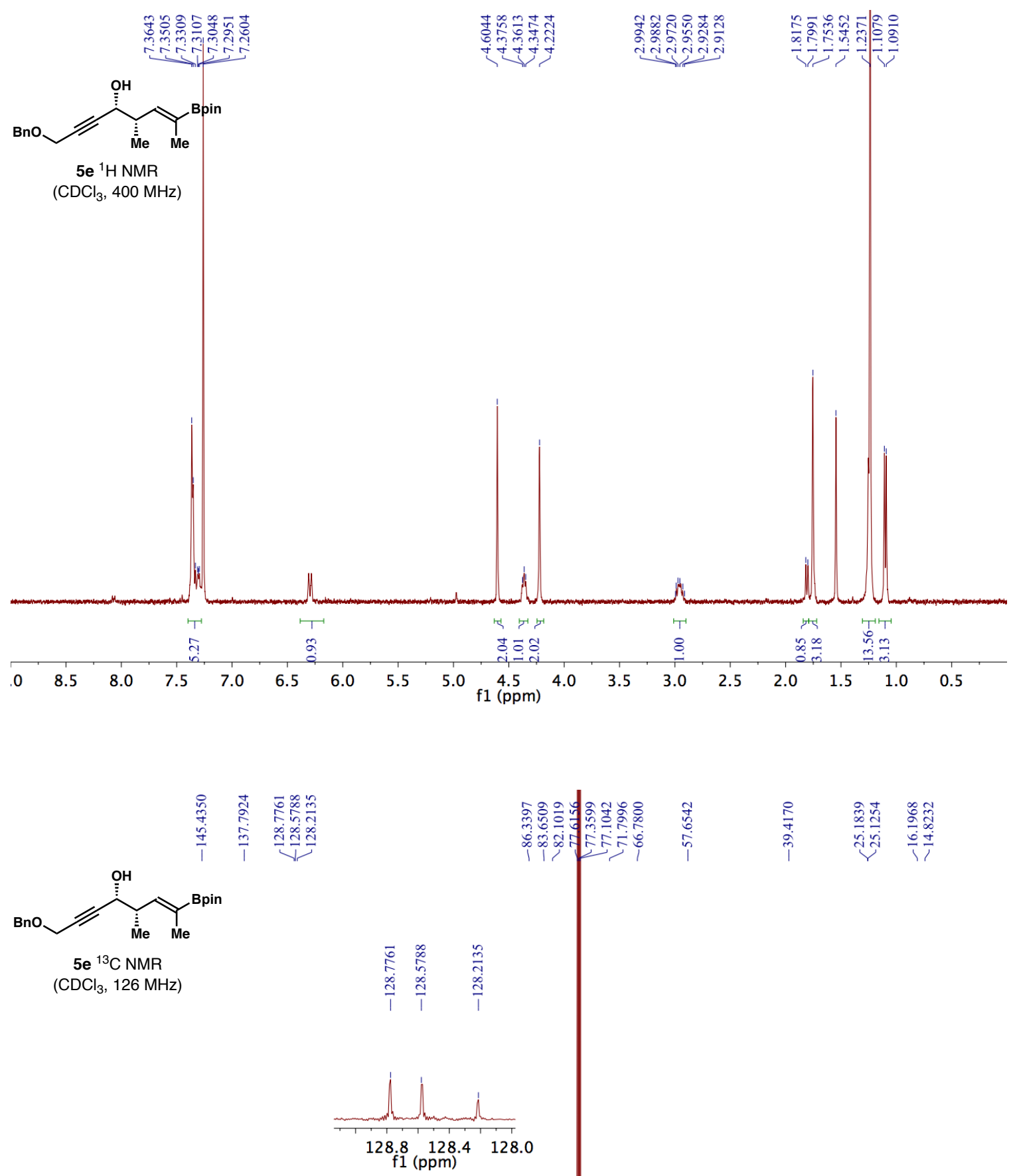

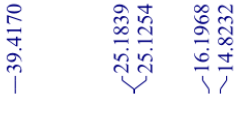

$5 e^{13} \mathrm{C}$ NMR

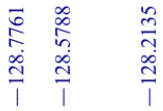
f1 (ppm)

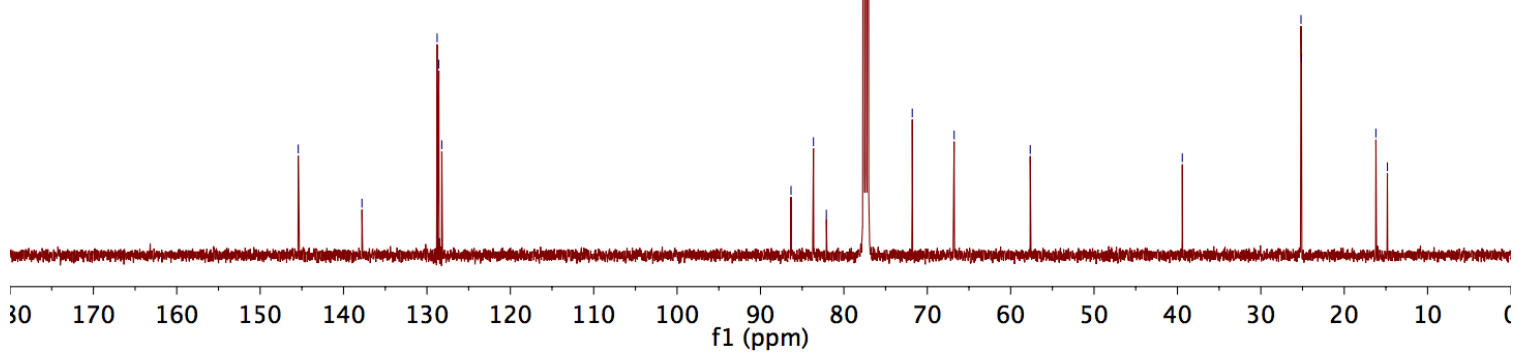



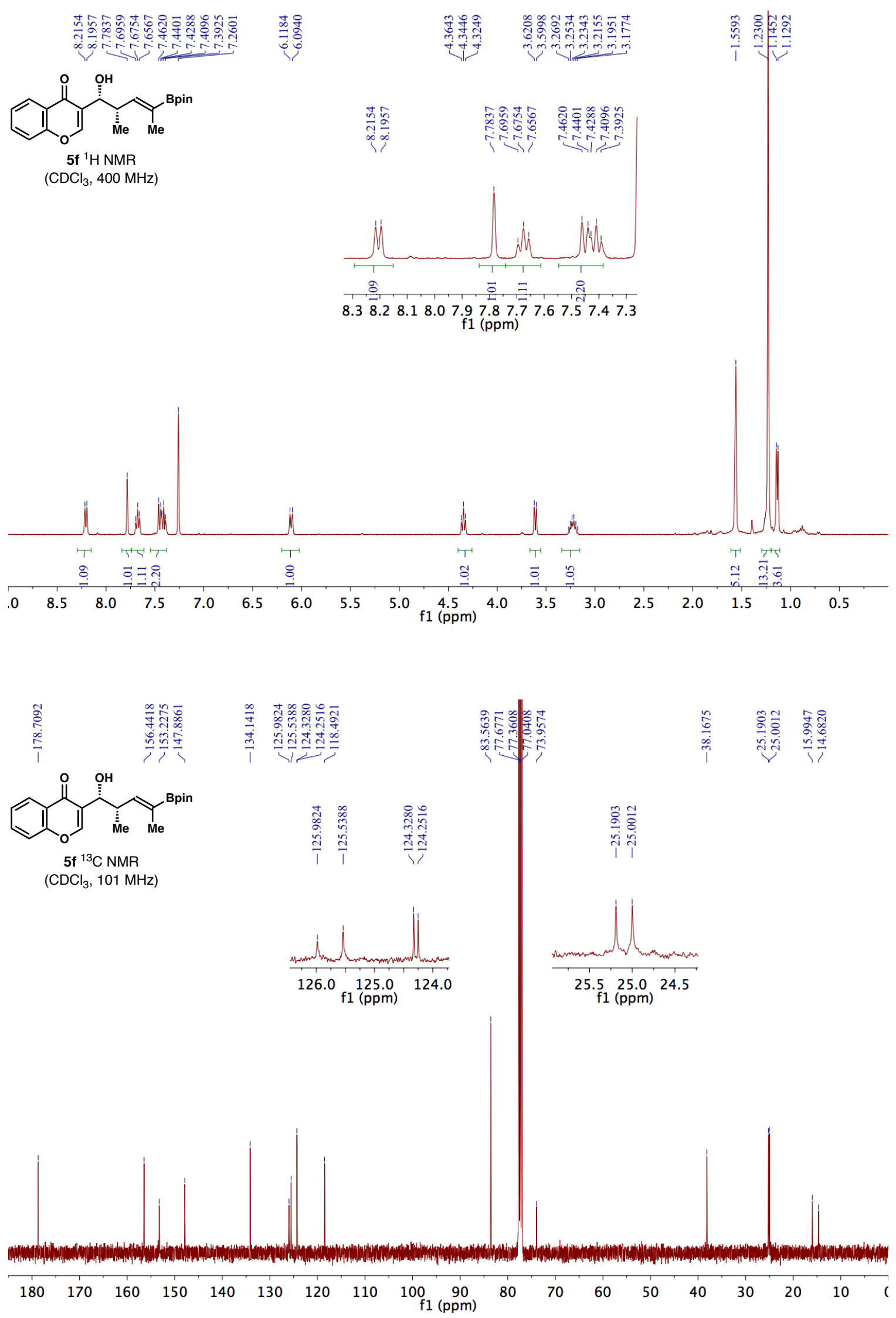

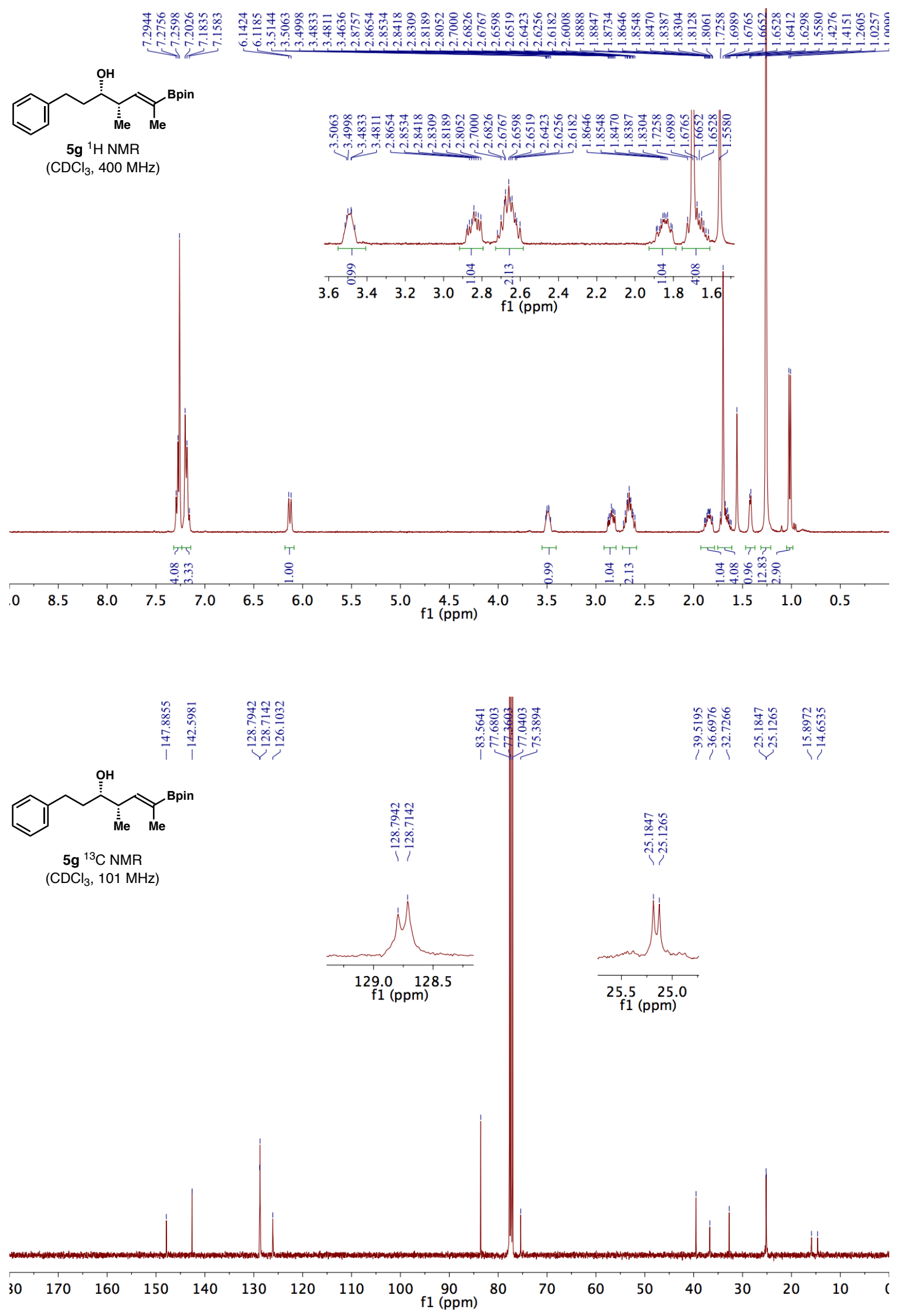

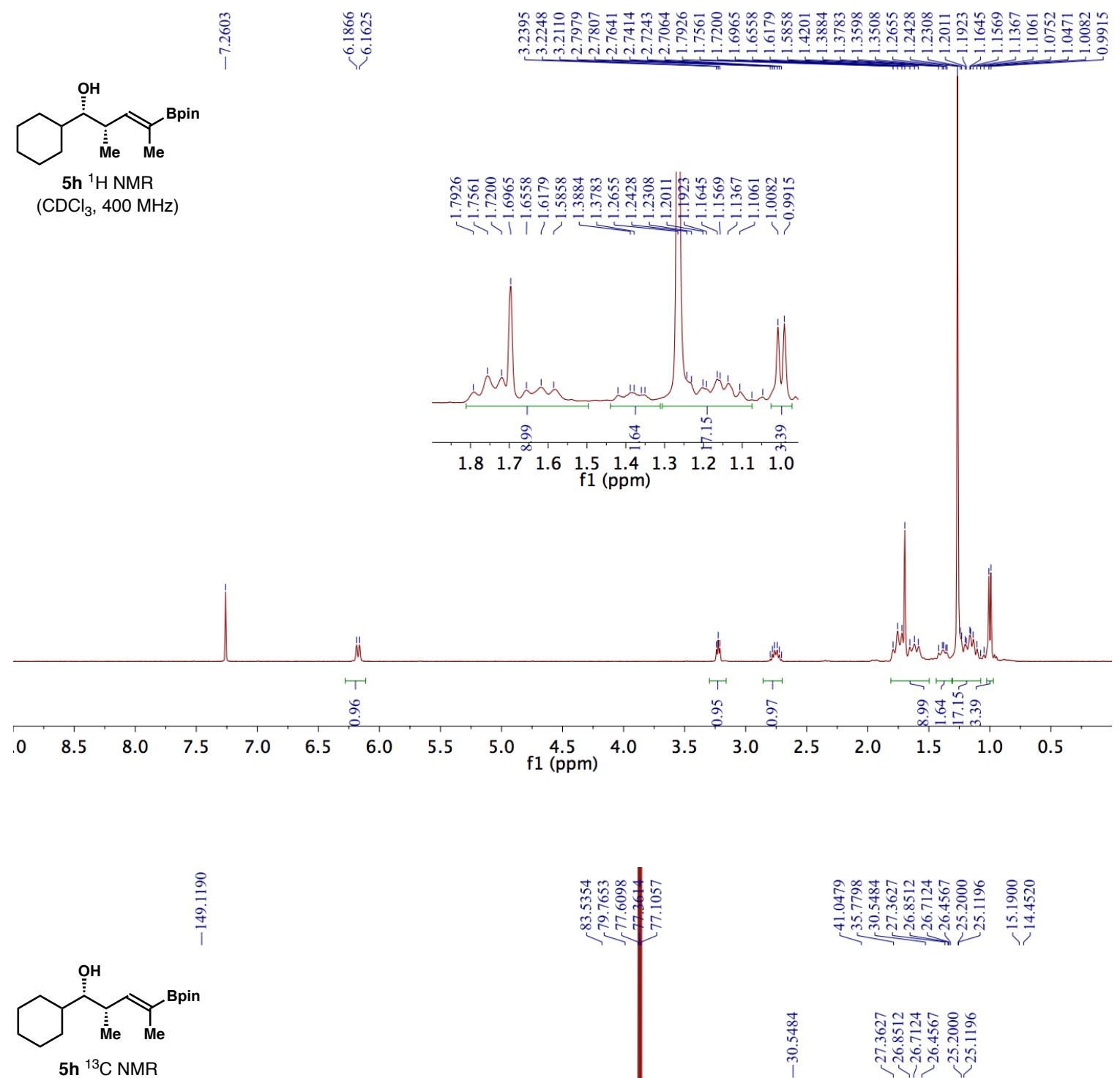
$\left(\mathrm{CDCl}_{3}, 101 \mathrm{MHz}\right)$
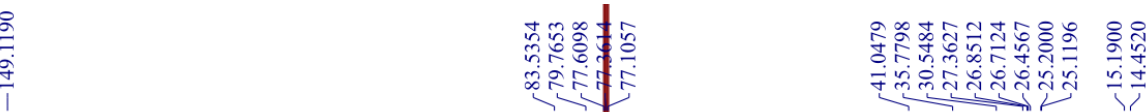

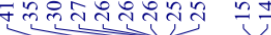
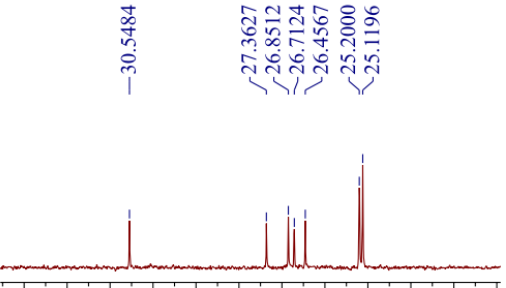

$\begin{array}{llllllllllll}33 & 32 & 31 & 30 & 29 & 28 & 27 & 26 & 25 & 24 & 23 & 22\end{array}$ f1 (ppm)

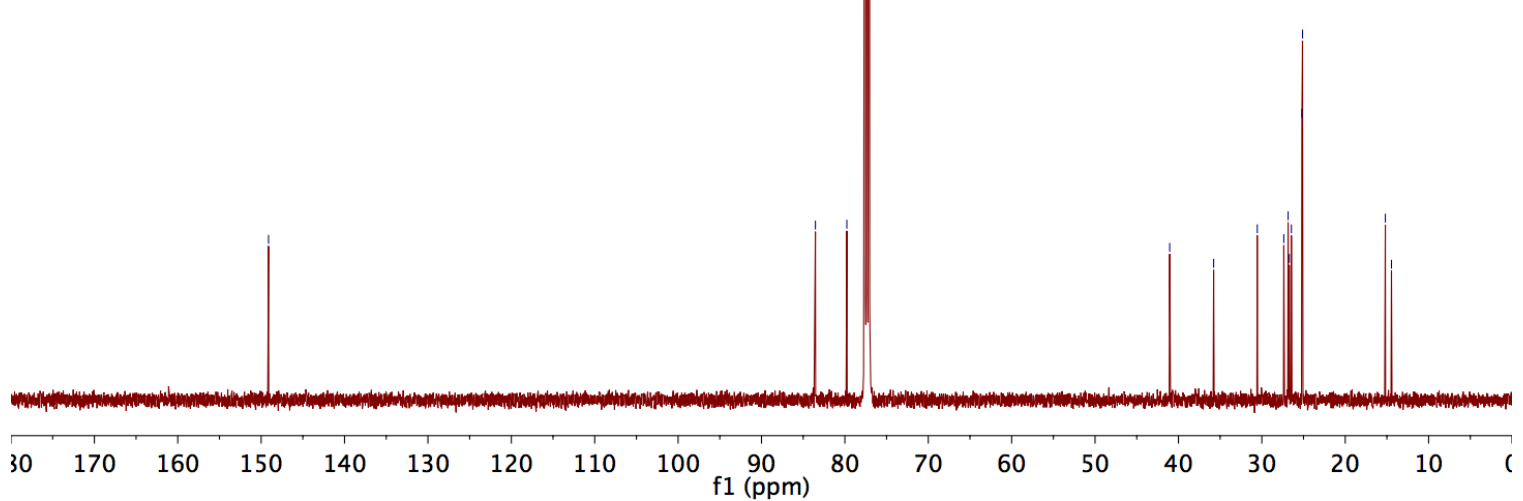



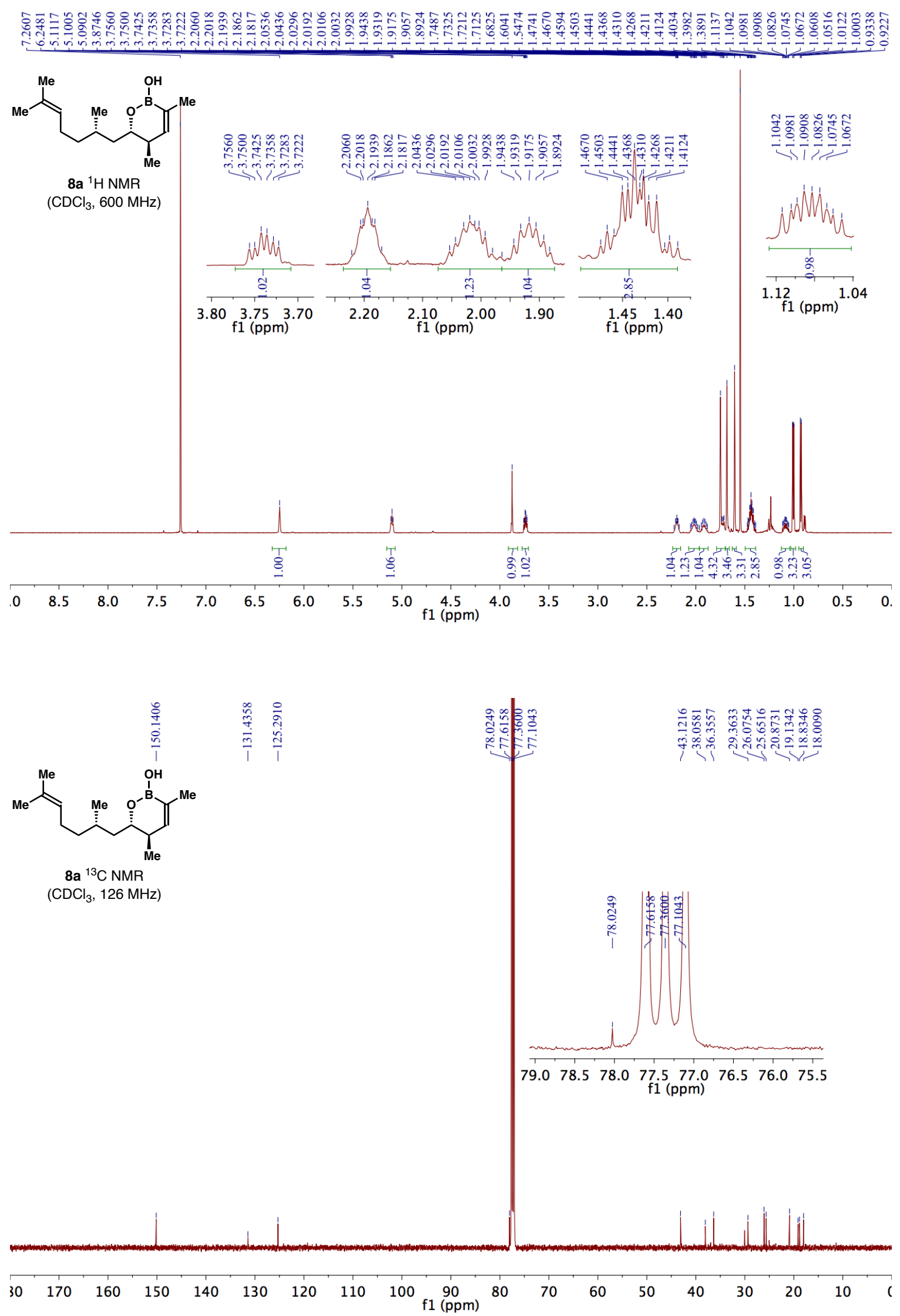
<smiles>CC(C)=CCC[C@H](C)CC1OB(O)C(C)=CC1C</smiles>

$8 b^{1} \mathrm{H}$ NMR $\left(\mathrm{CDCl}_{3}, 400 \mathrm{MHz}\right)$

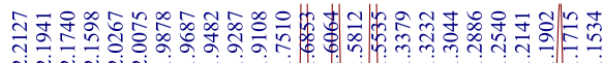

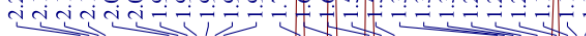

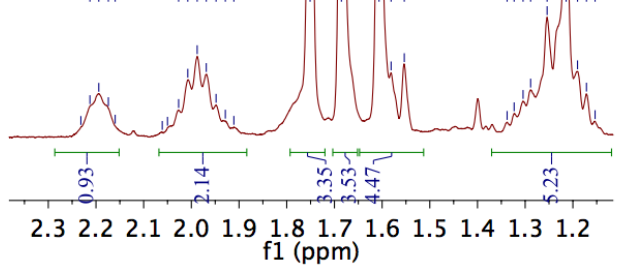

$\mathrm{f} 1(\mathrm{ppm})$

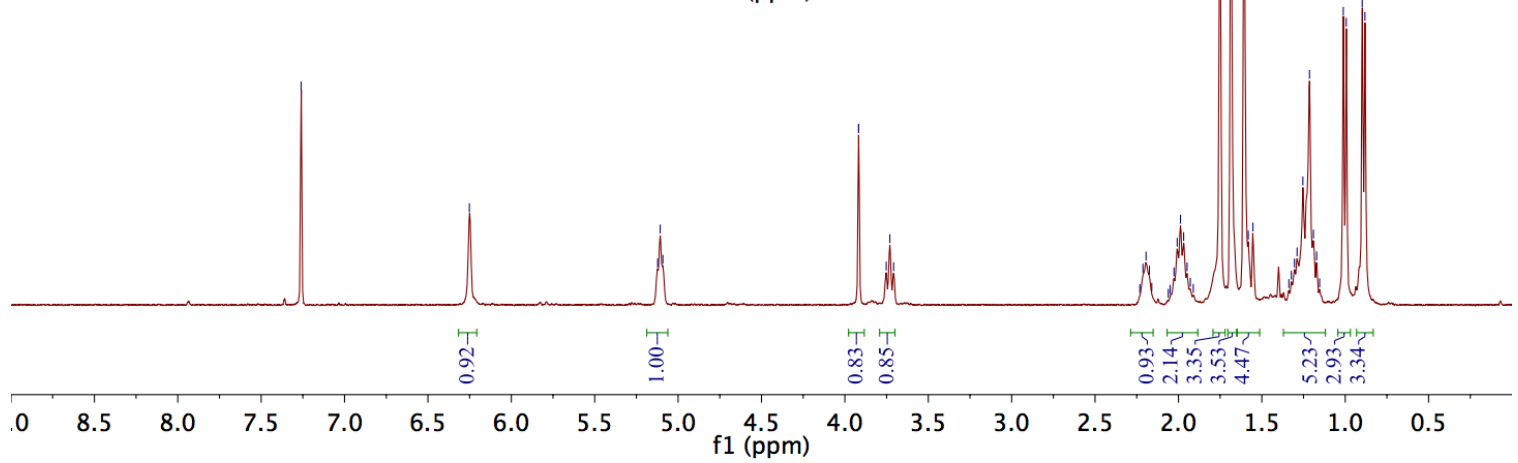

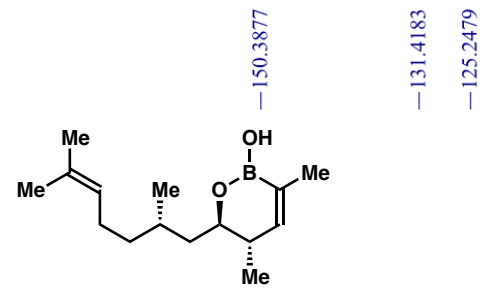

8b ${ }^{13} \mathrm{C} \mathrm{NMR}$

$\left(\mathrm{CDCl}_{3}, 101 \mathrm{MHz}\right)$
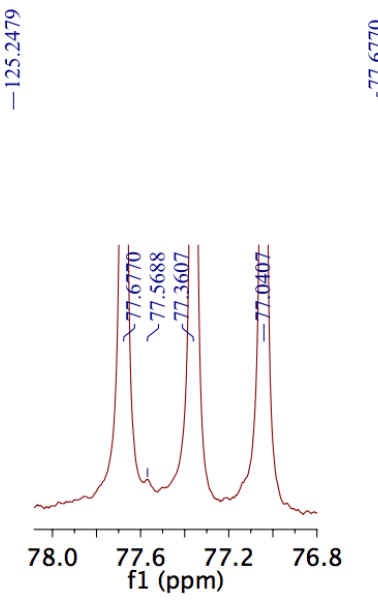
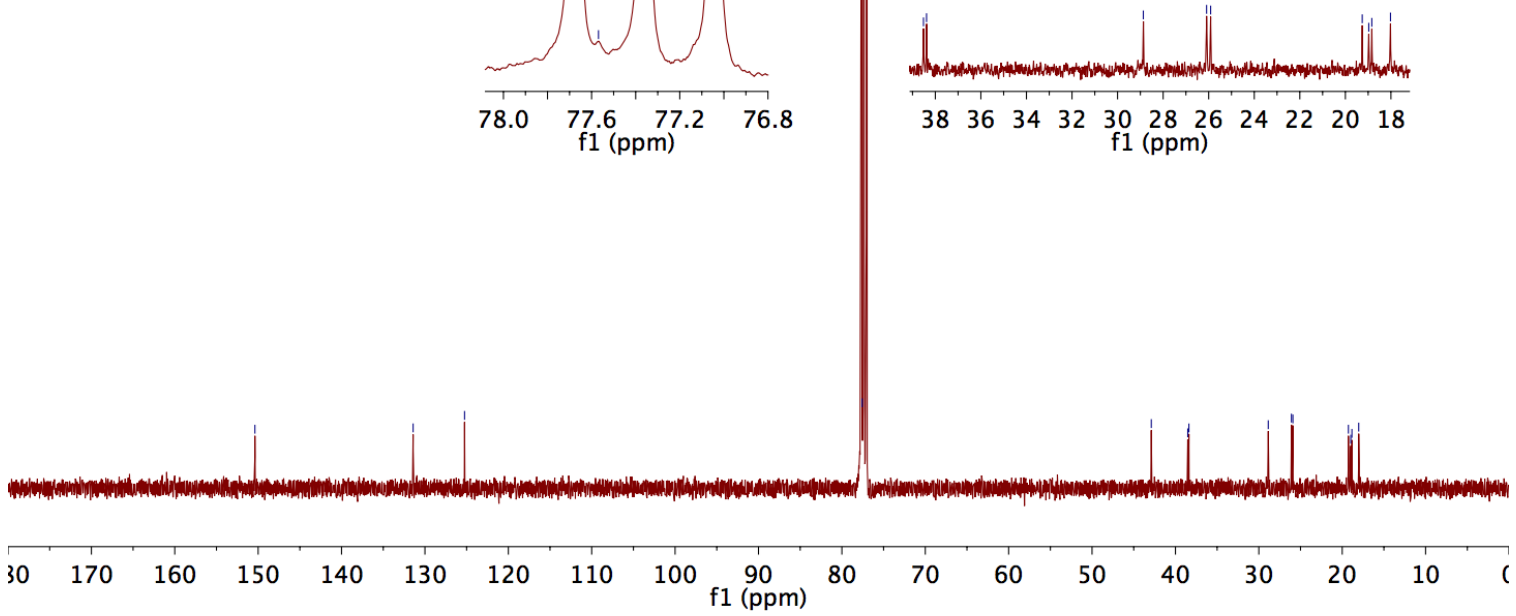

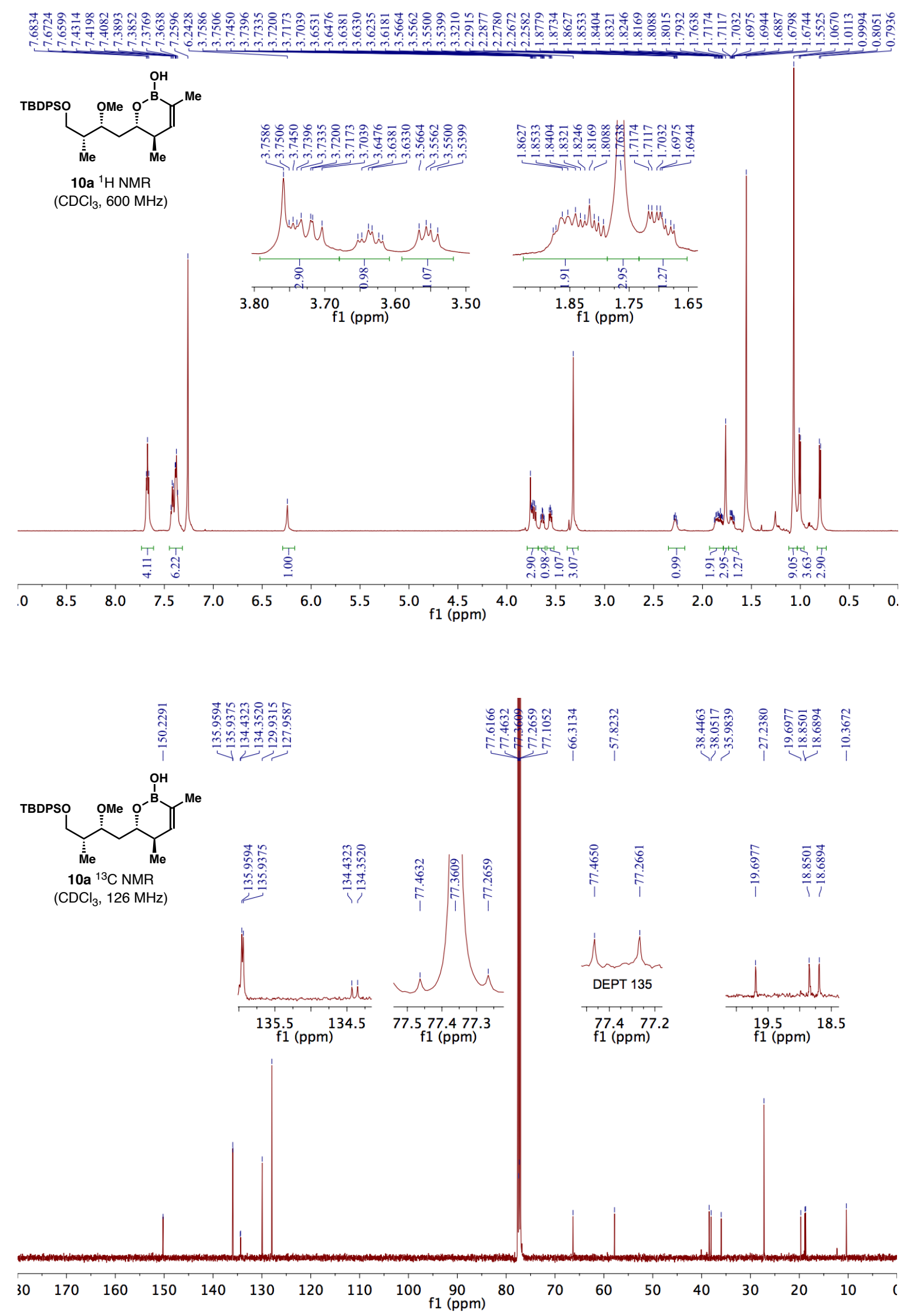

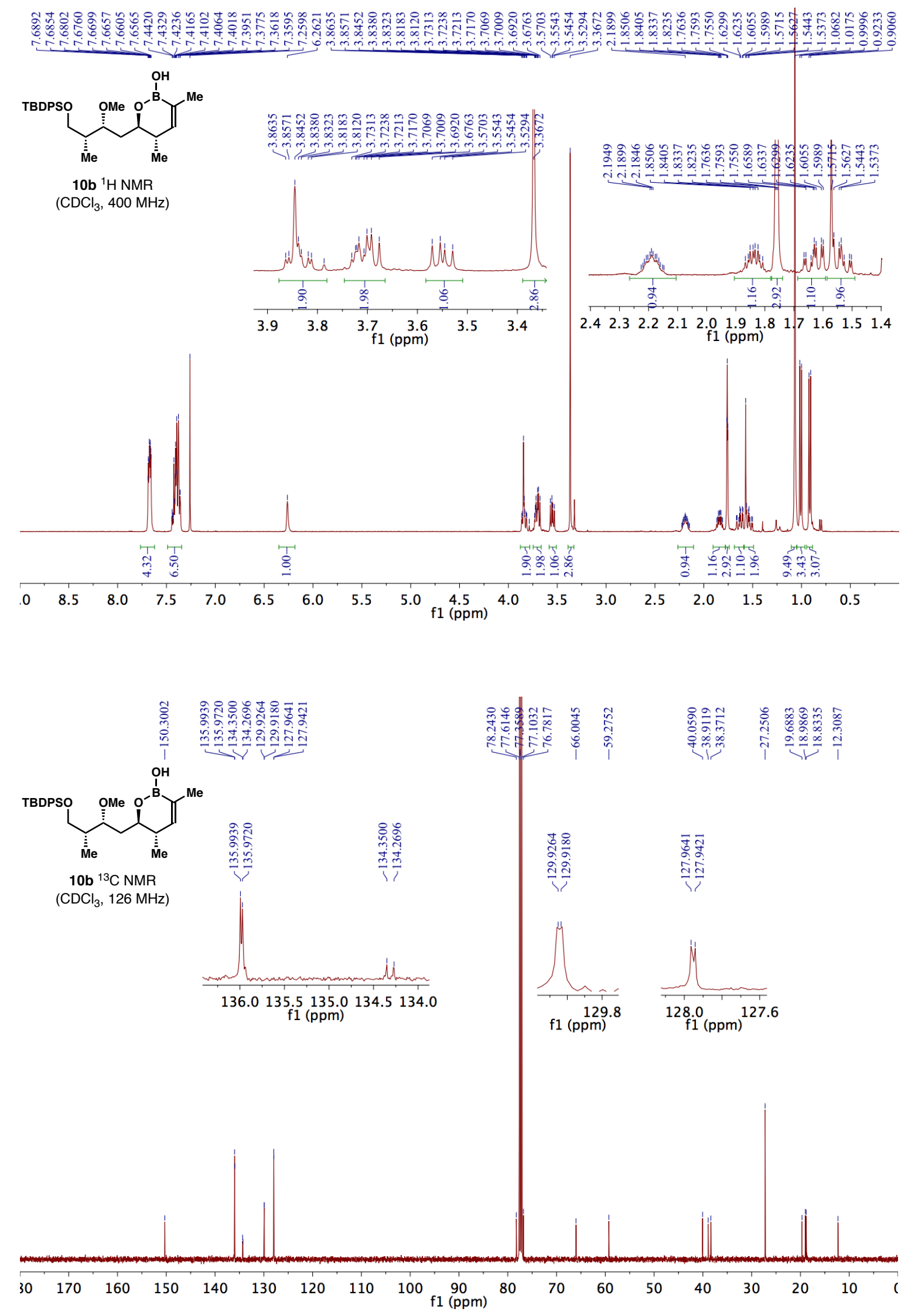

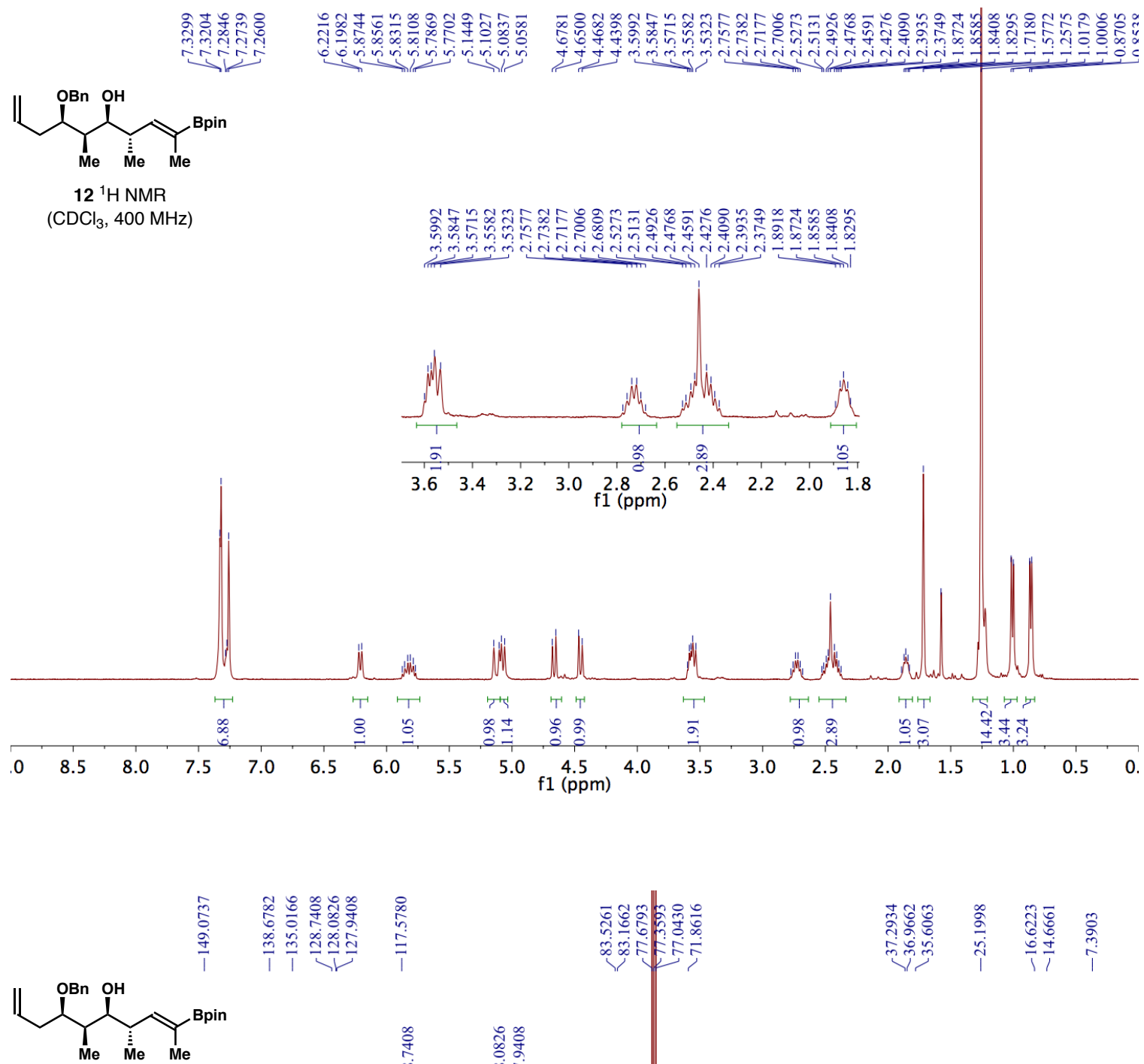

$12{ }^{13} \mathrm{C}$ NMR $\left(\mathrm{CDCl}_{3}, 101 \mathrm{MHz}\right)$
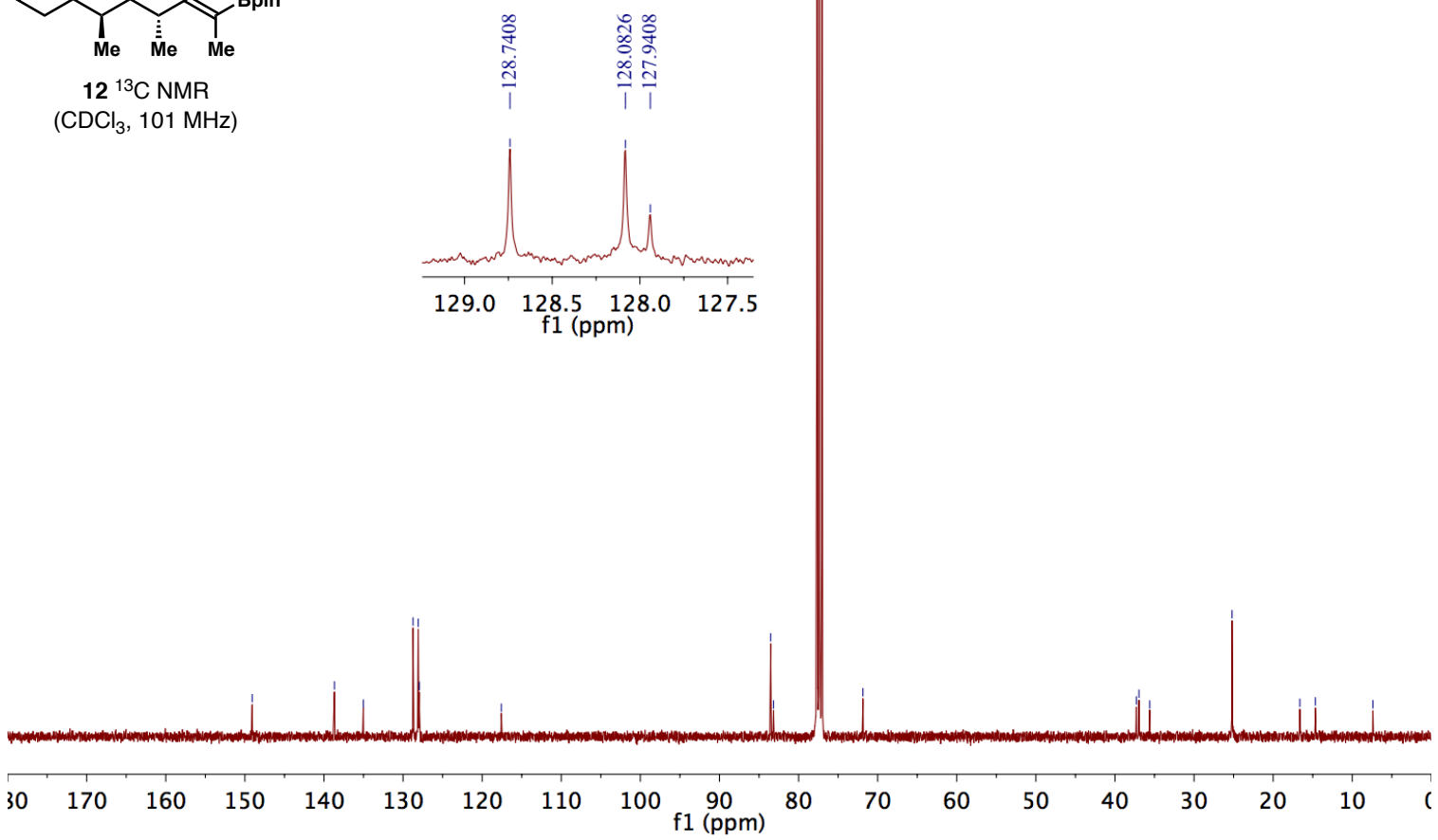

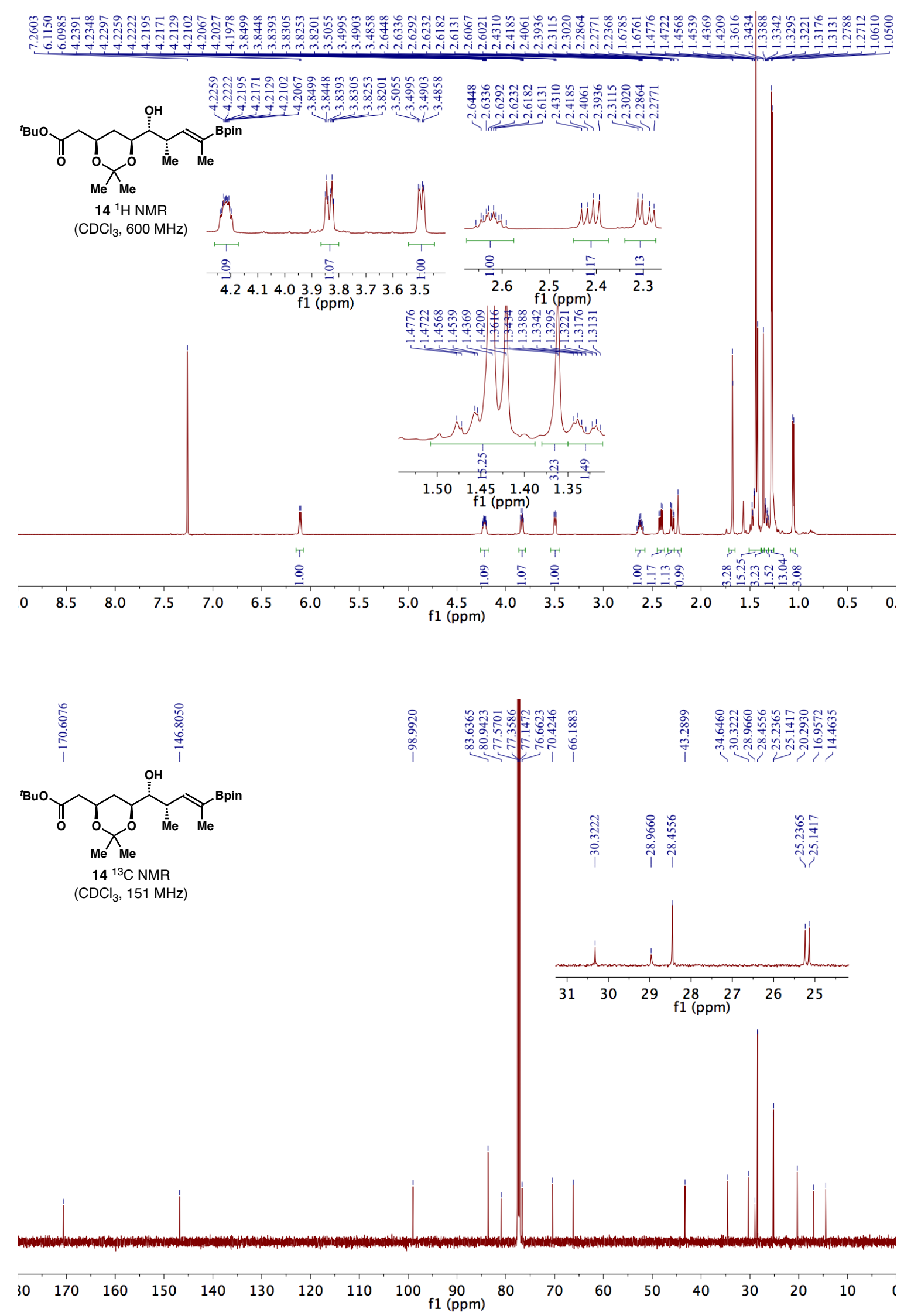

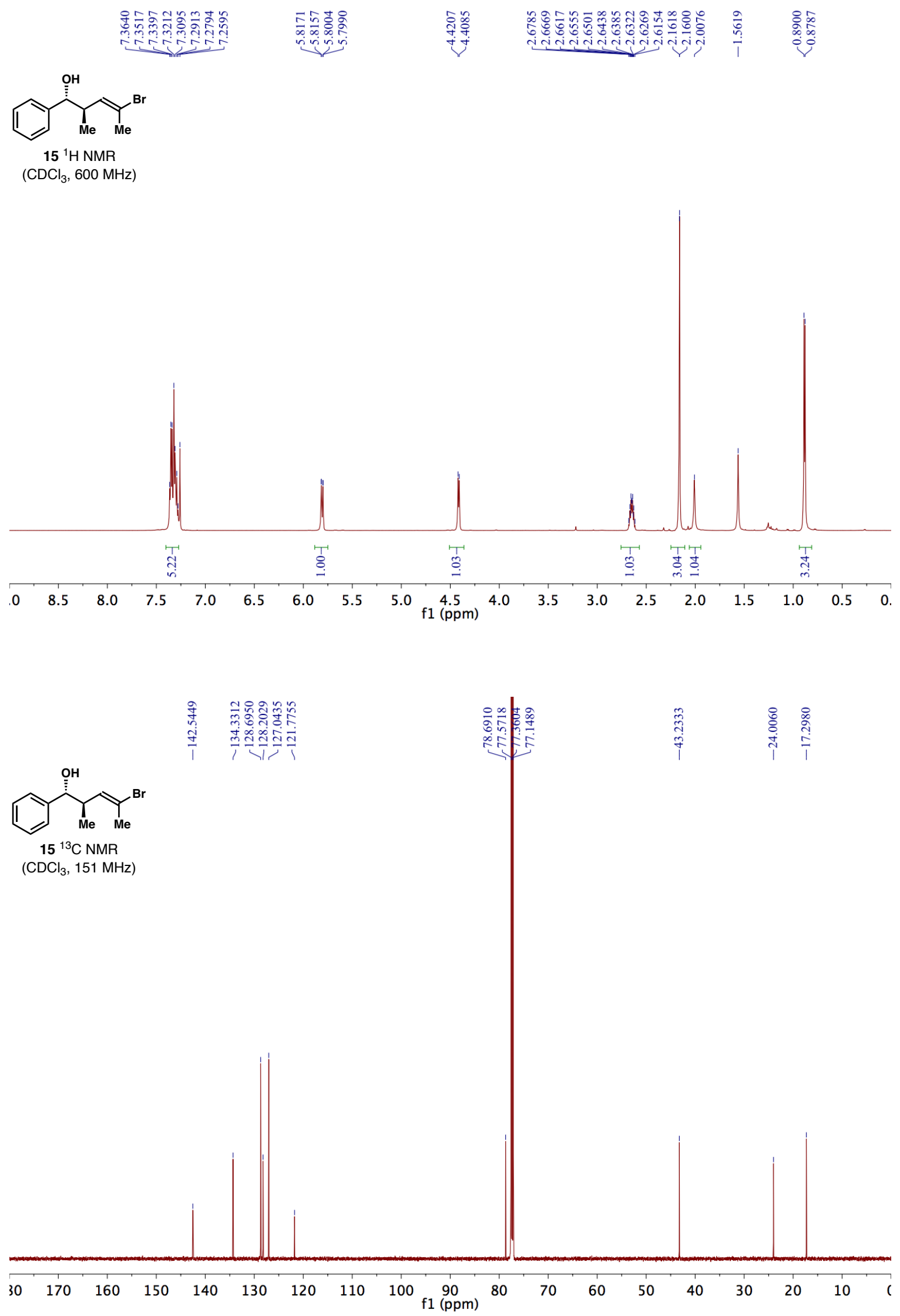

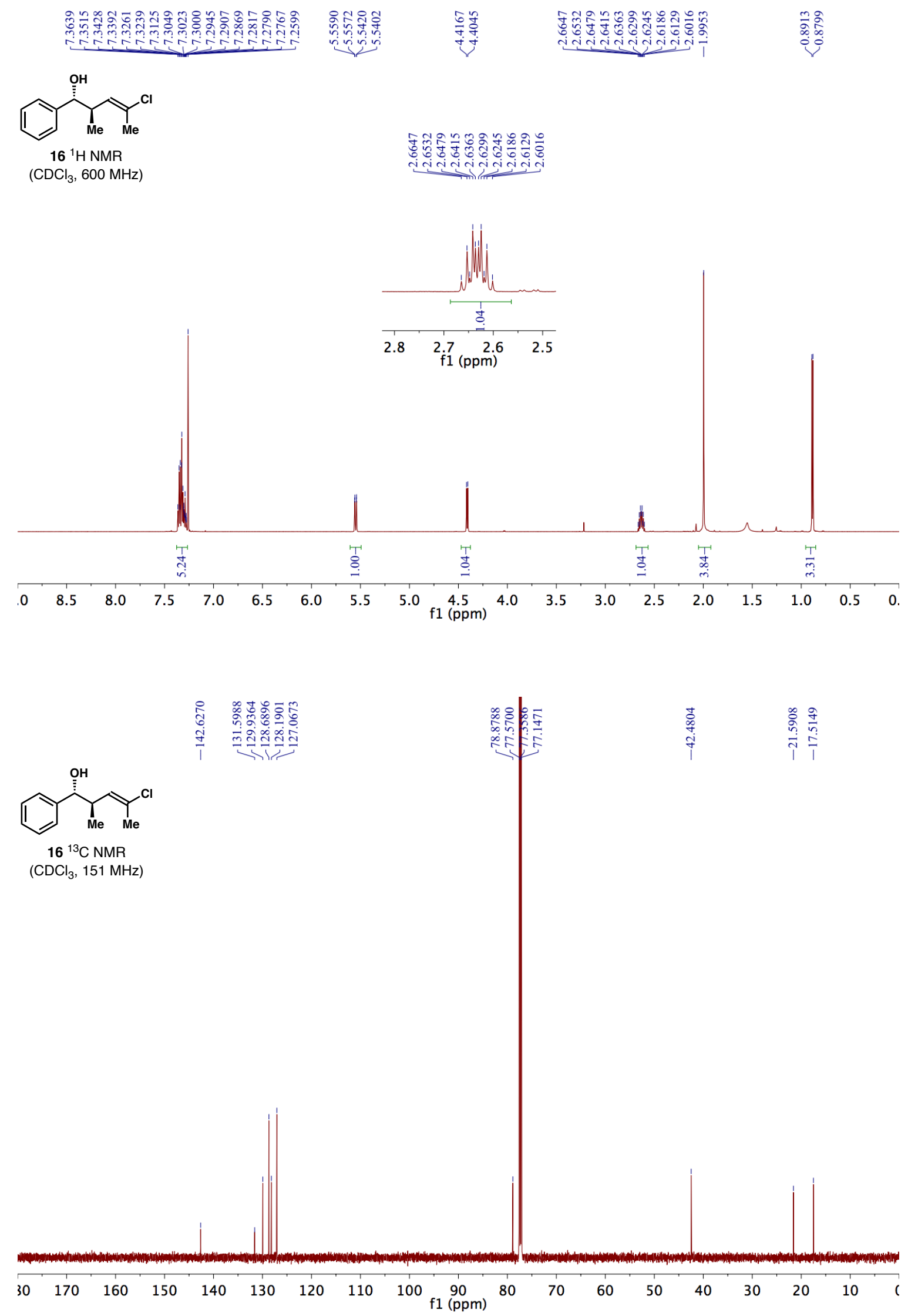

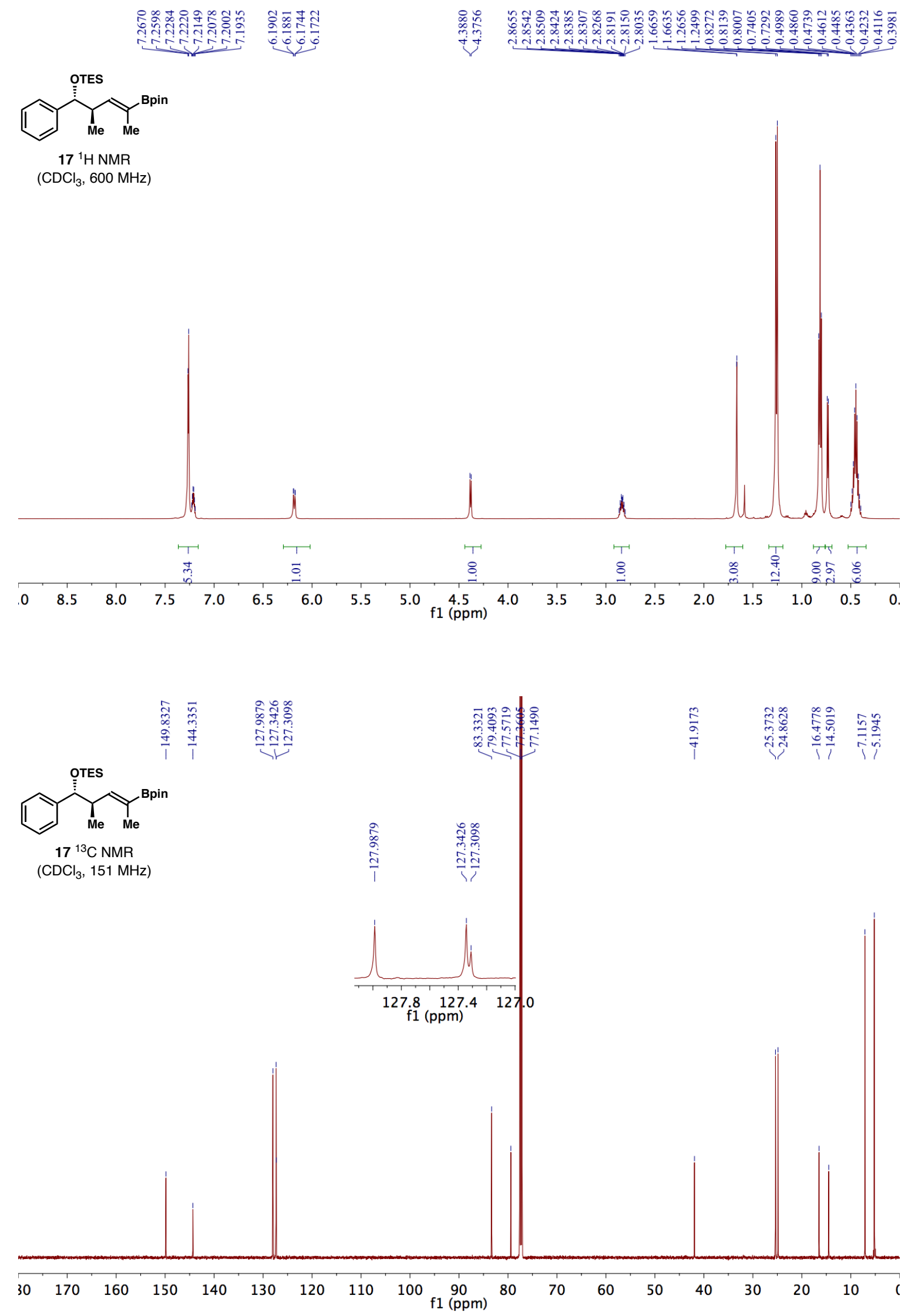

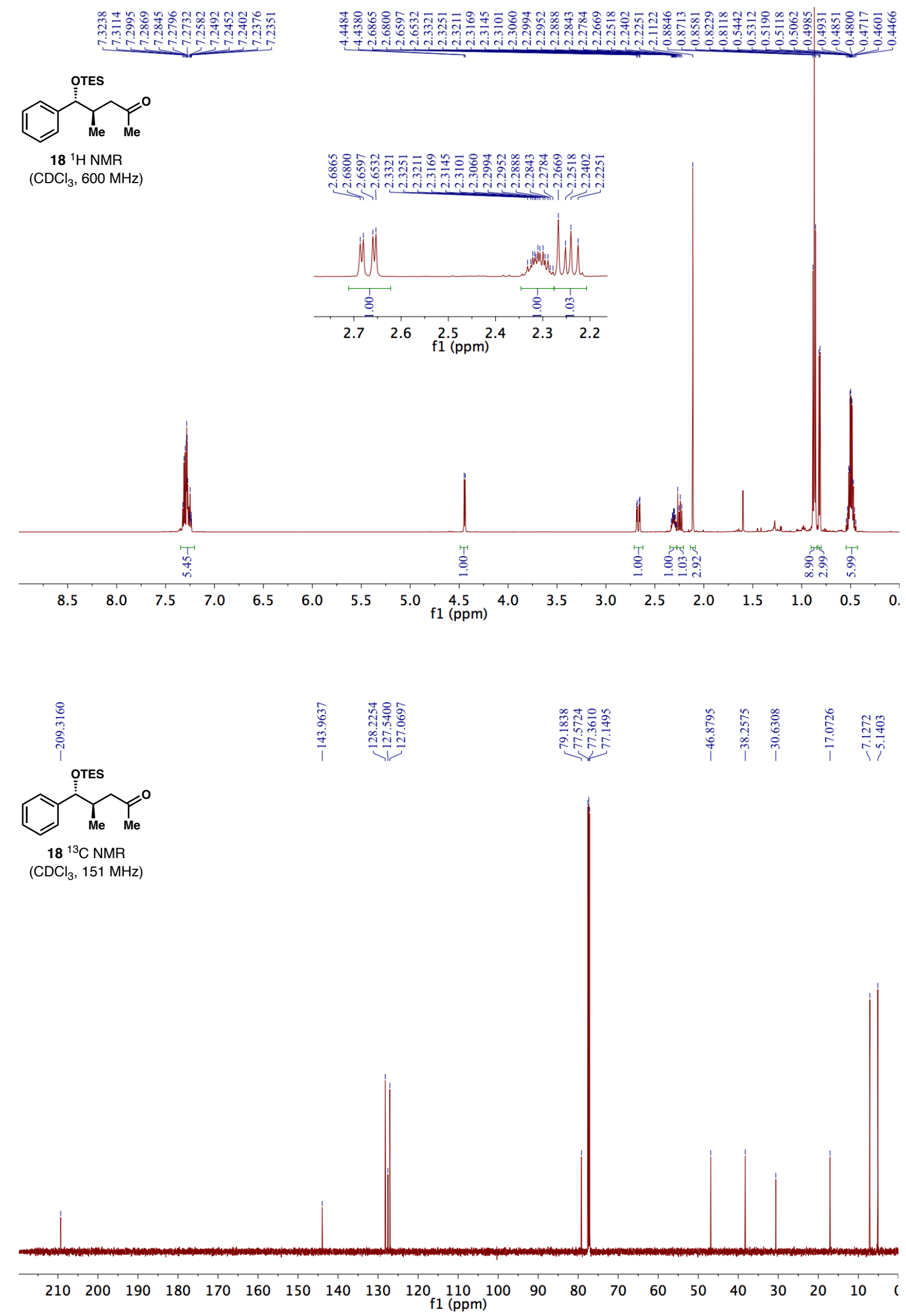

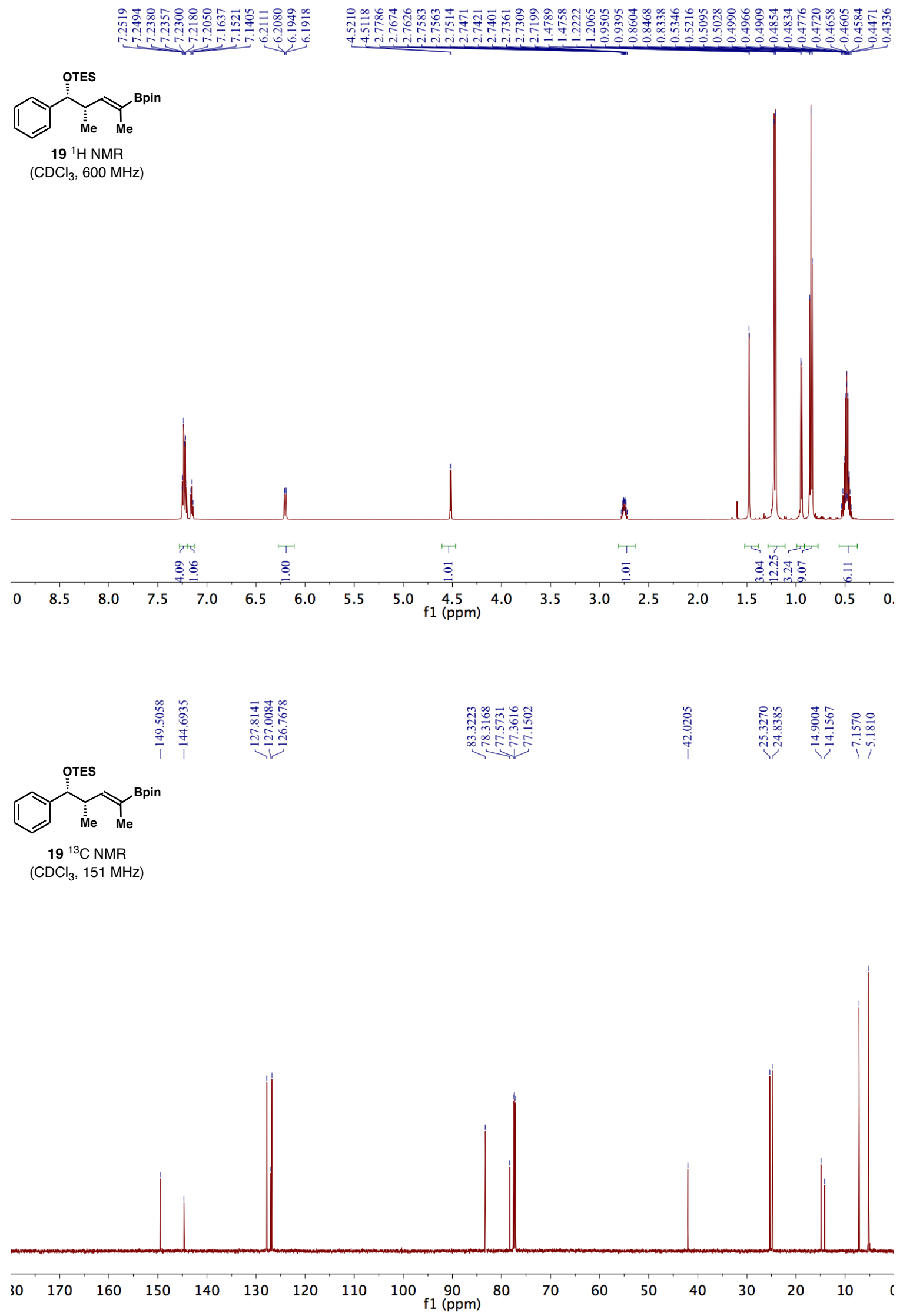

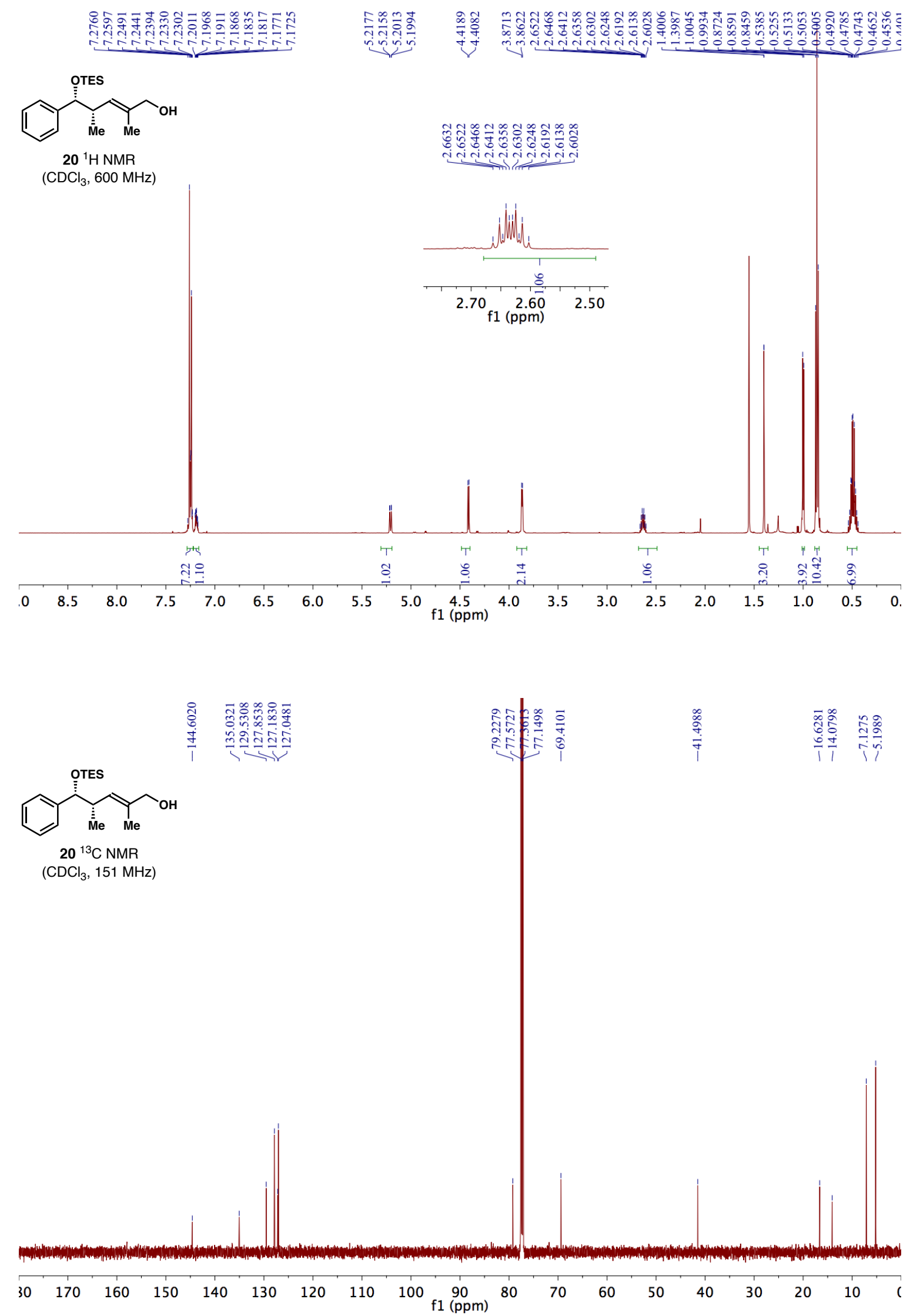

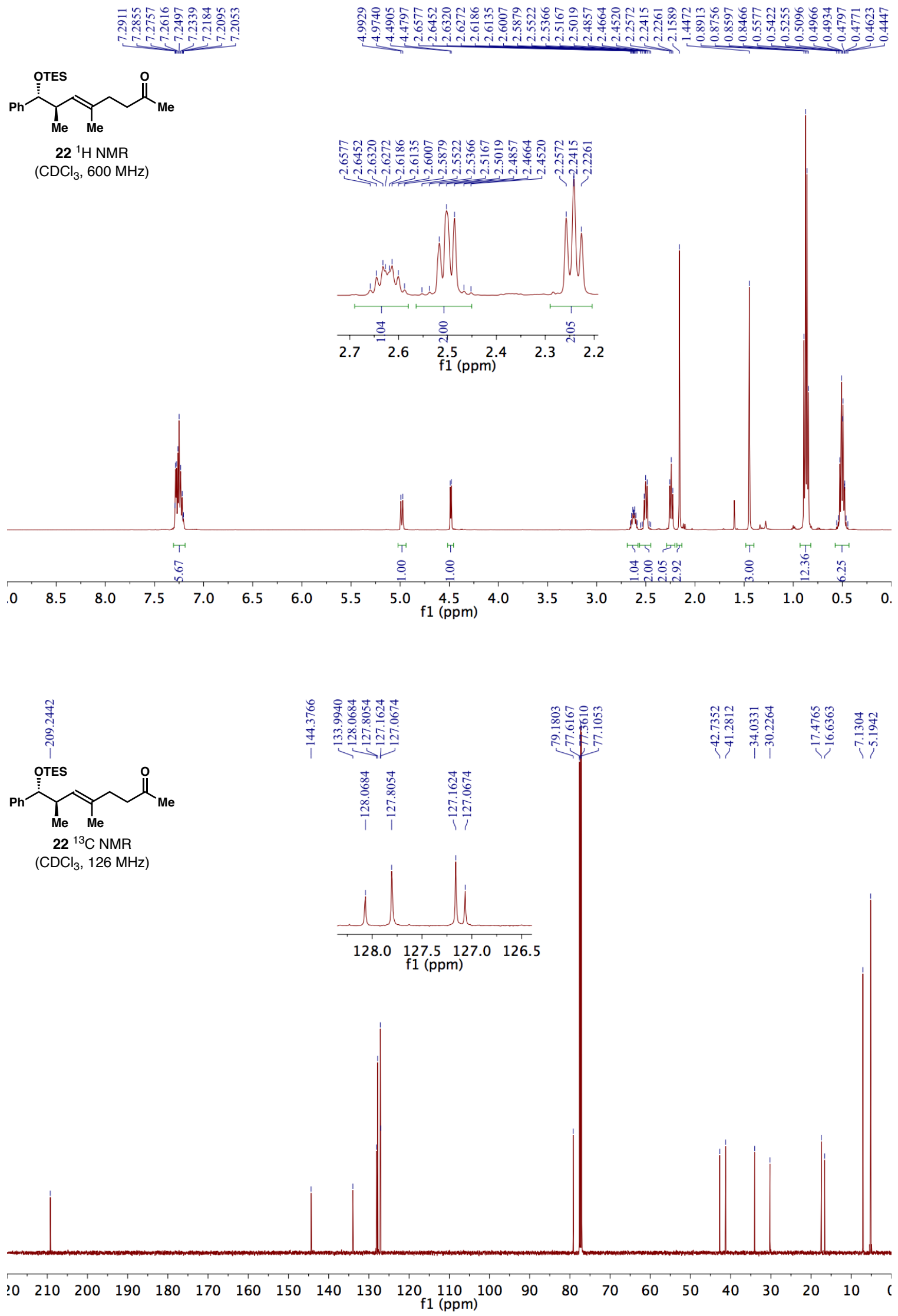

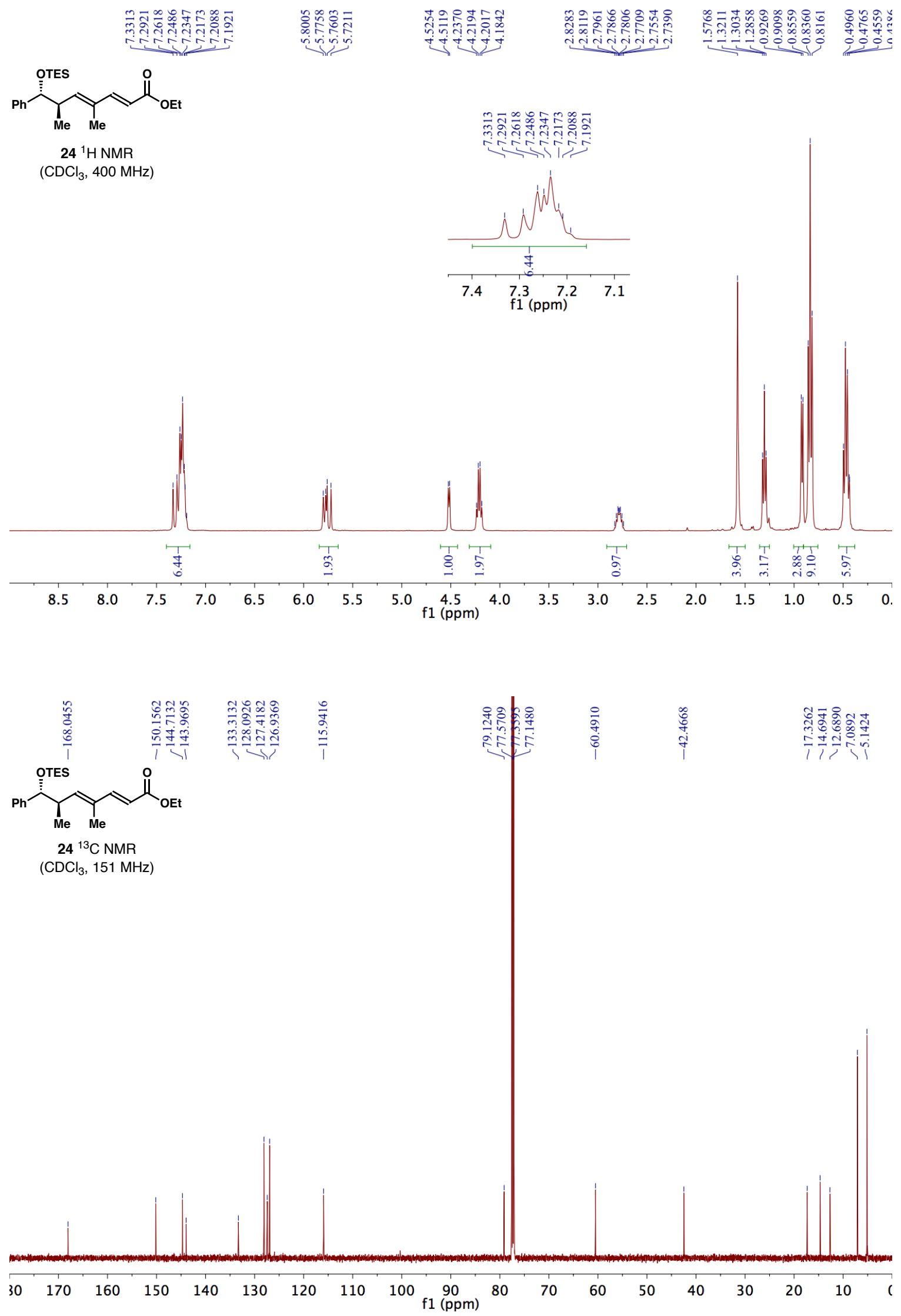

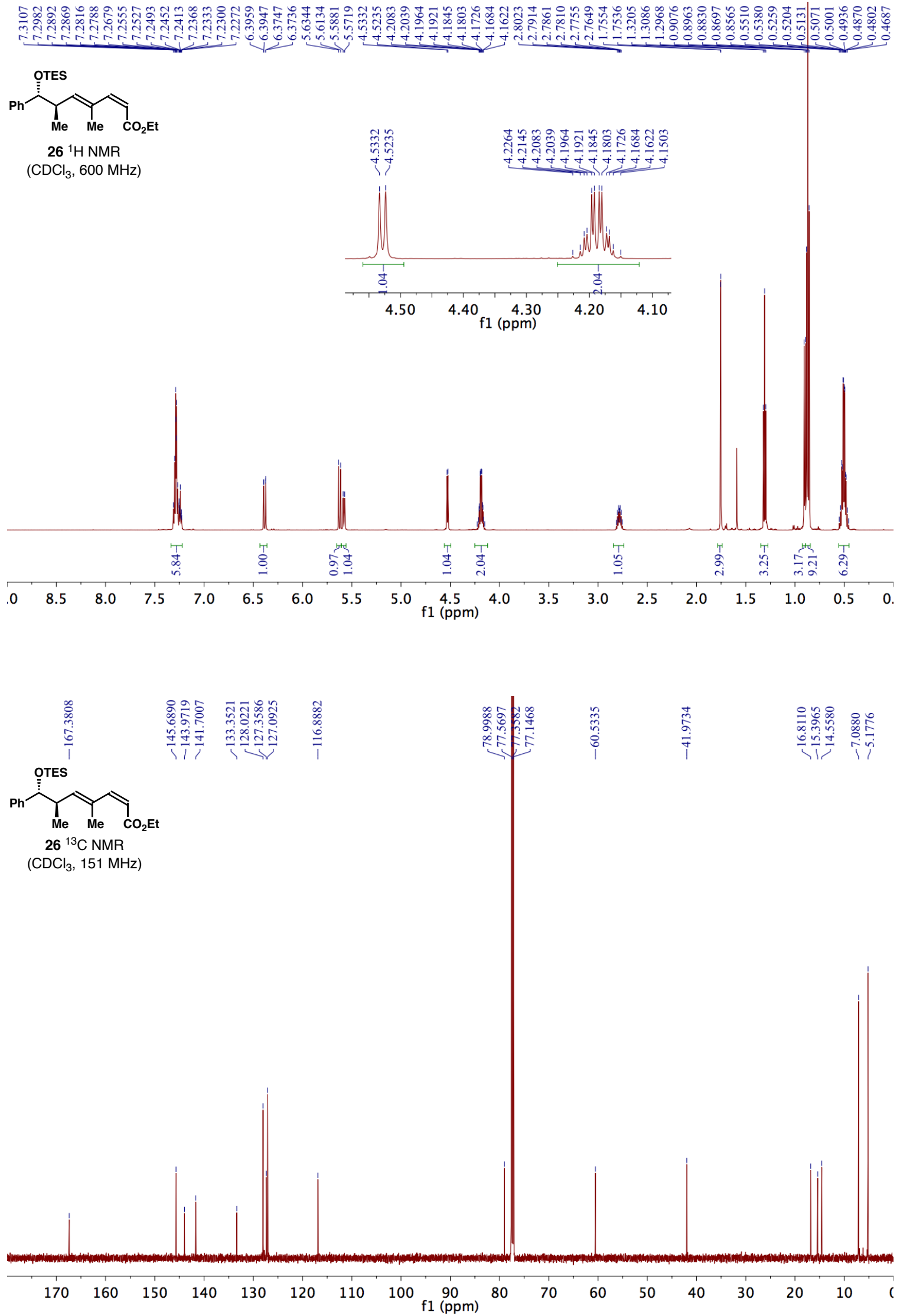

SI-110 

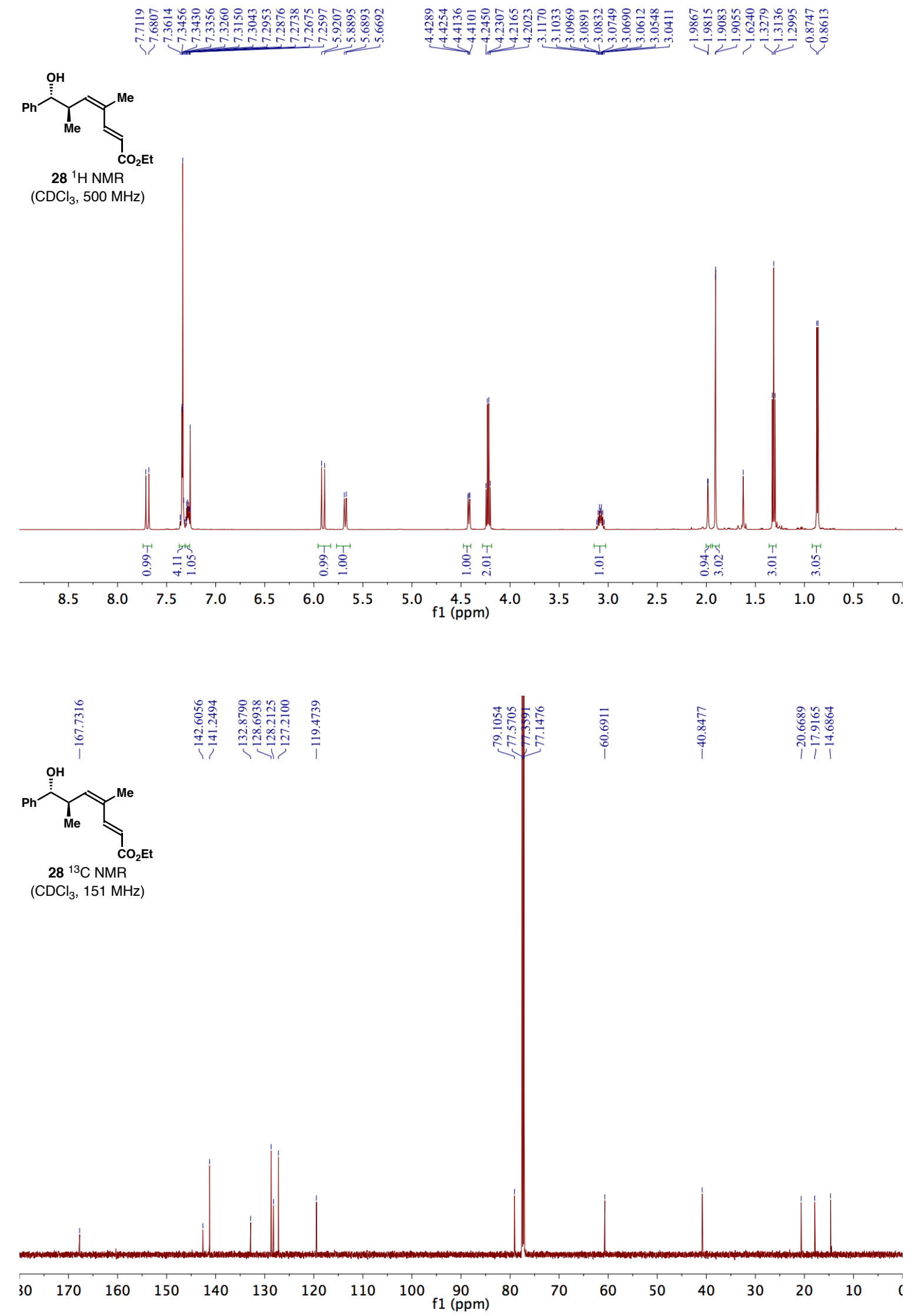


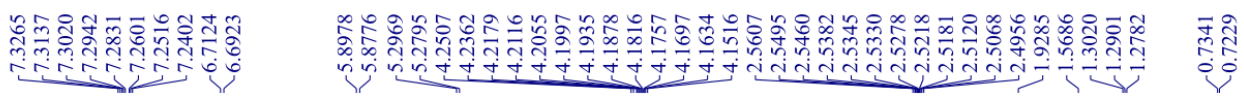

$\underbrace{\mathrm{OHe}}_{\mathrm{Me}}$

$29^{1} \mathrm{H}$ NMR

$\left(\mathrm{CDCl}_{3}, 600 \mathrm{MHz}\right)$
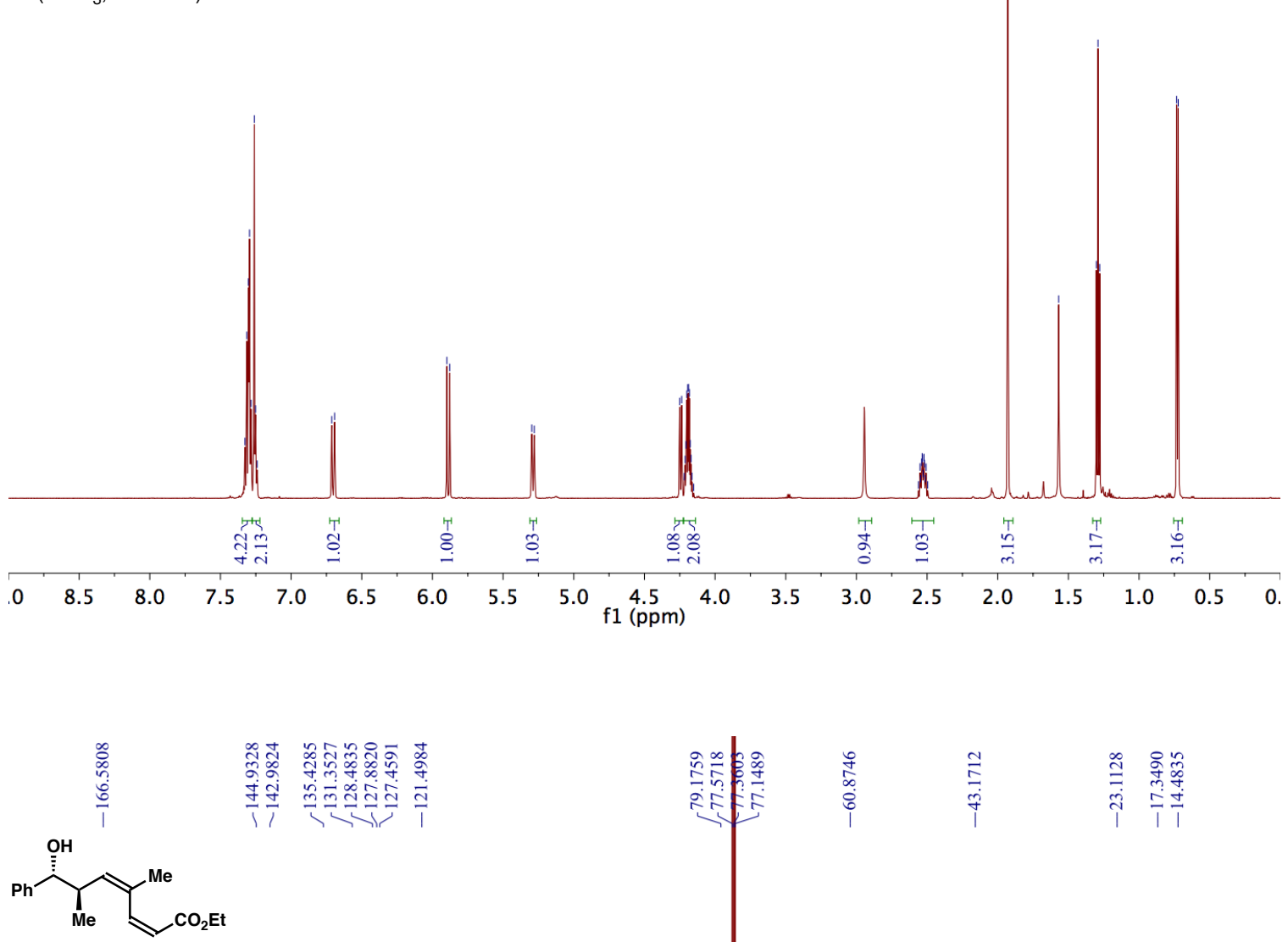

$29{ }^{13} \mathrm{C}$ NMR

$\left(\mathrm{CDCl}_{3}, 151 \mathrm{MHz}\right)$

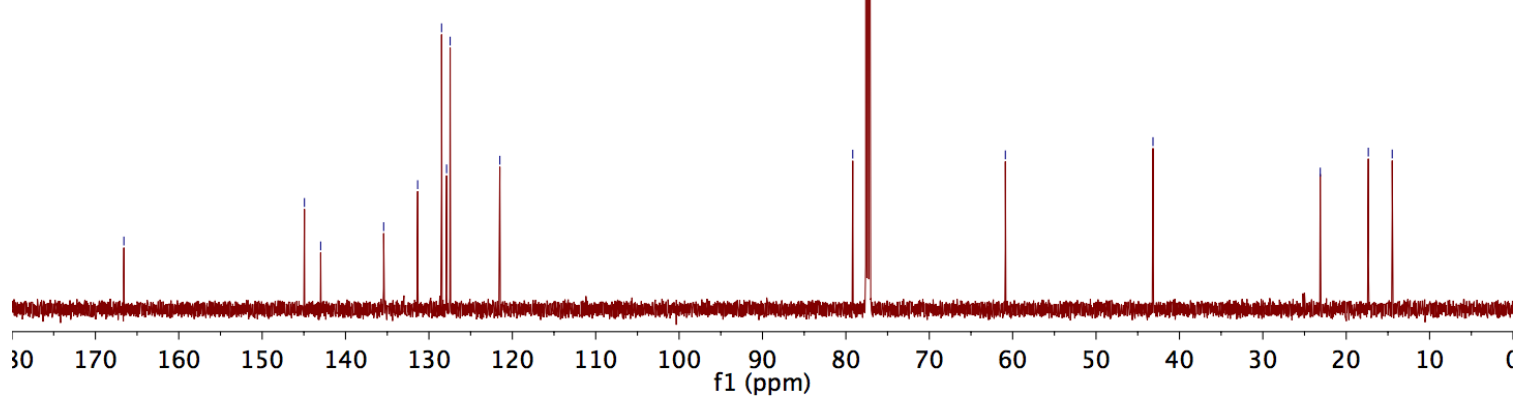

SI-112 

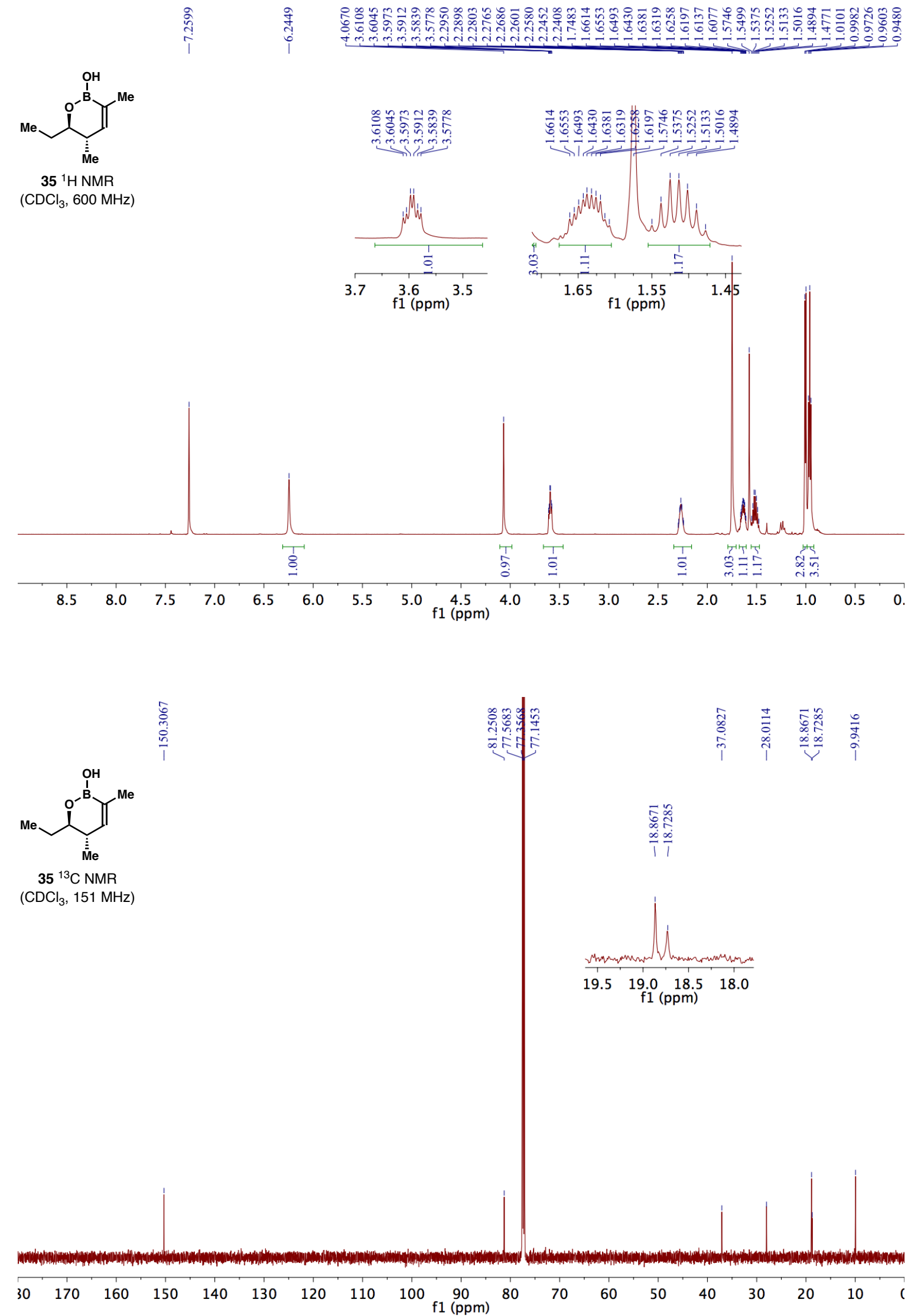

$35{ }^{13} \mathrm{C}$ NMR $\left(\mathrm{CDCl}_{3}, 151 \mathrm{MHz}\right)$ 

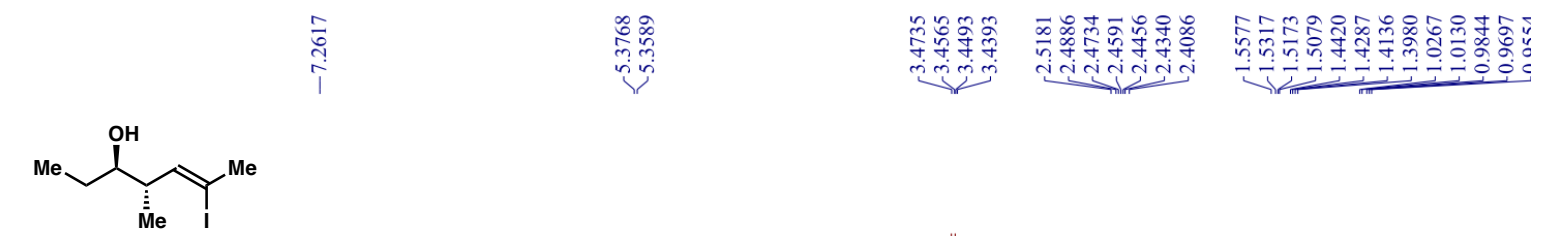

$33{ }^{1} \mathrm{H}$ NMR

$\left(\mathrm{CDCl}_{3}, 500 \mathrm{MHz}\right)$

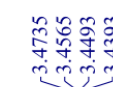

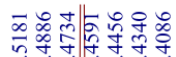

त त त i i

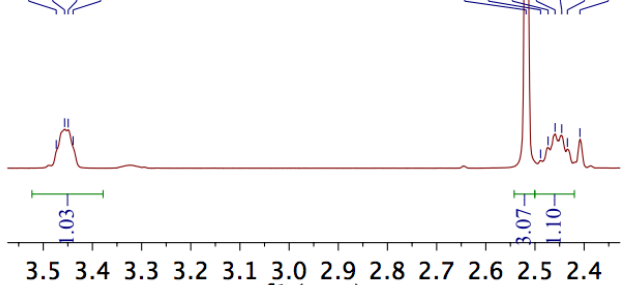

f1 (ppm)
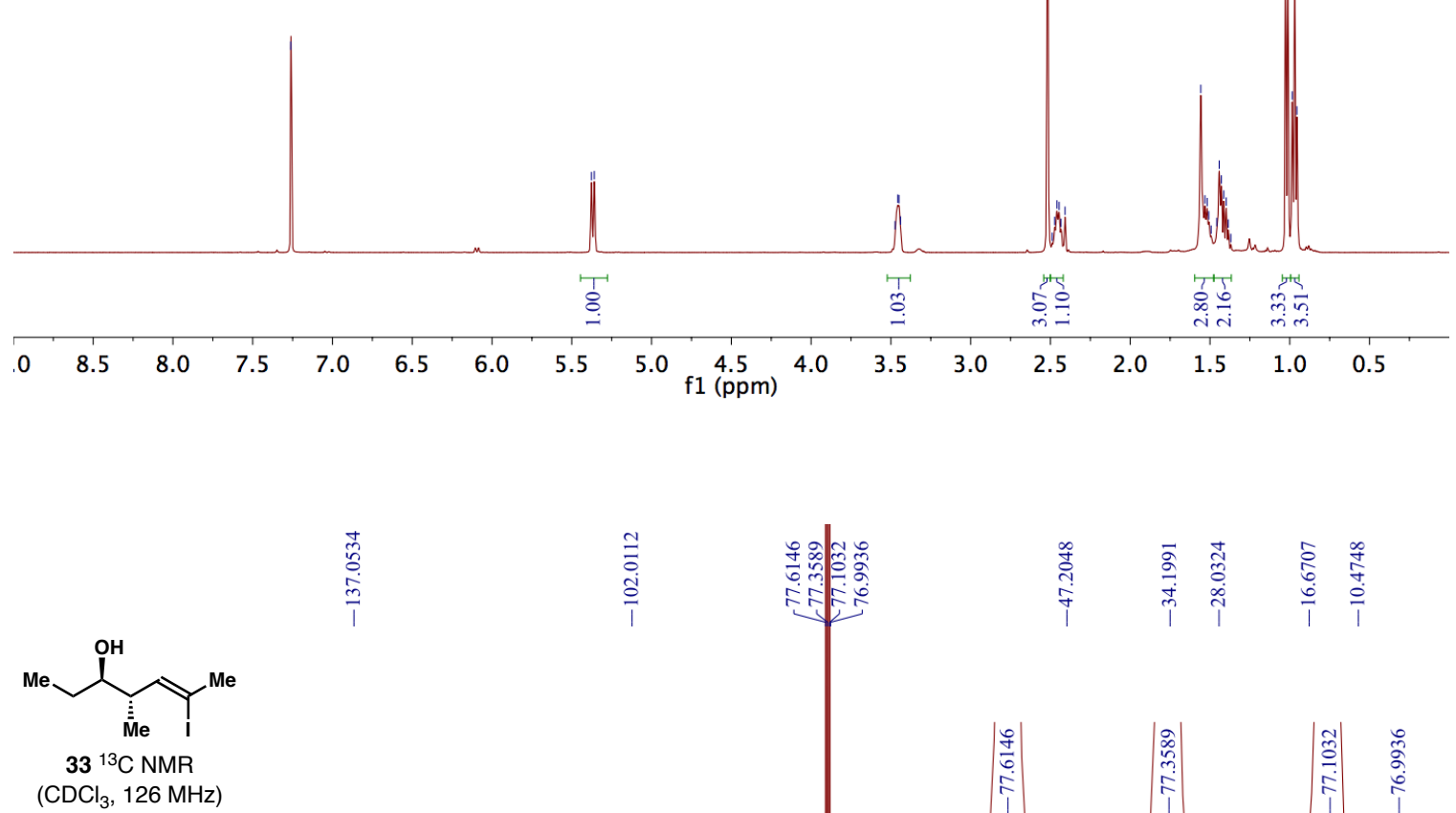

$\left(\mathrm{CDCl}_{3}, 126 \mathrm{MHz}\right)$
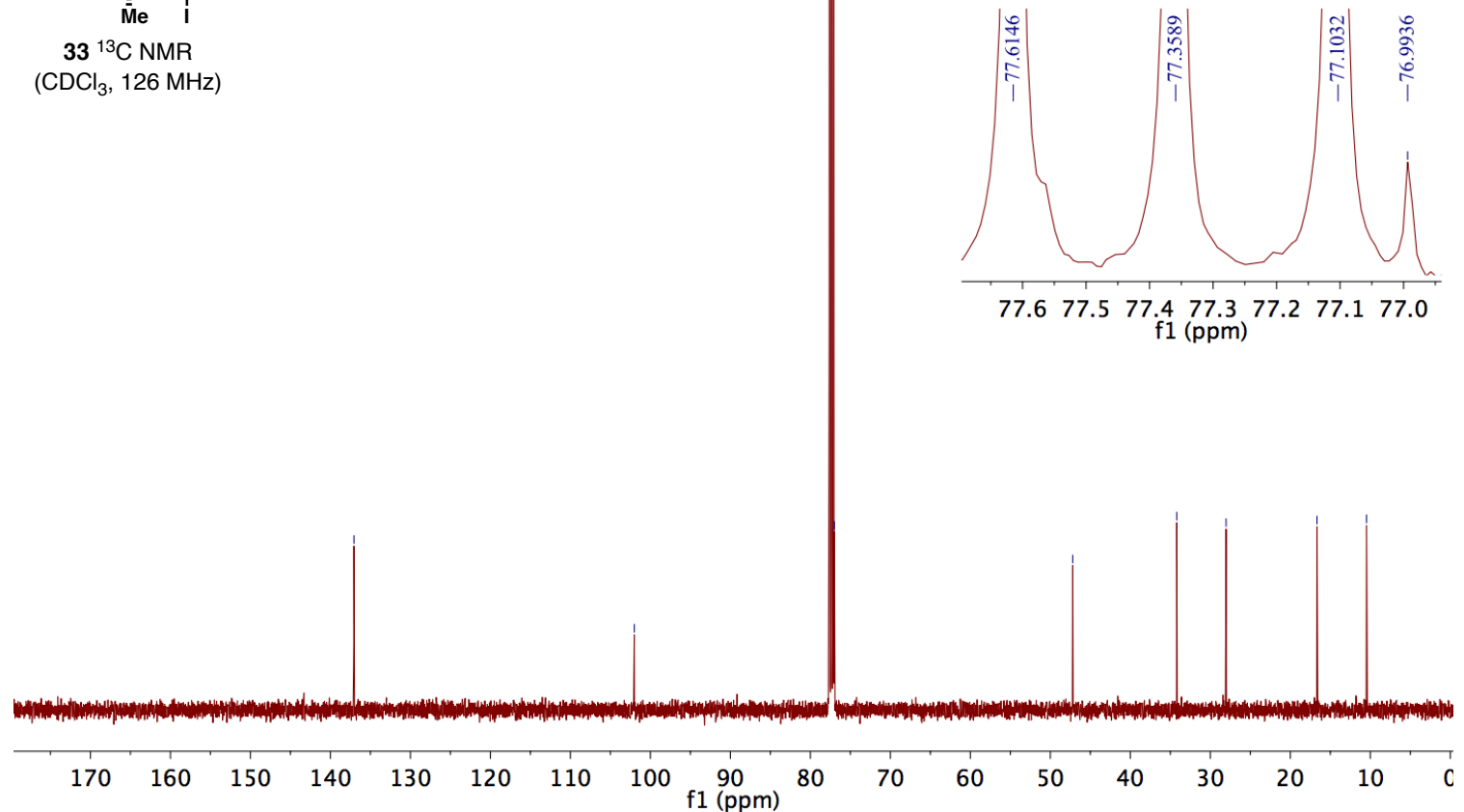


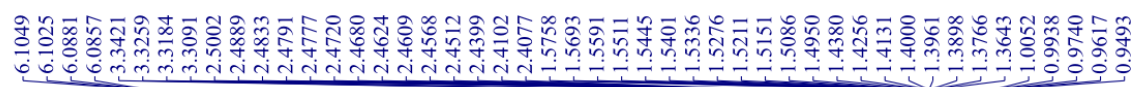

o.
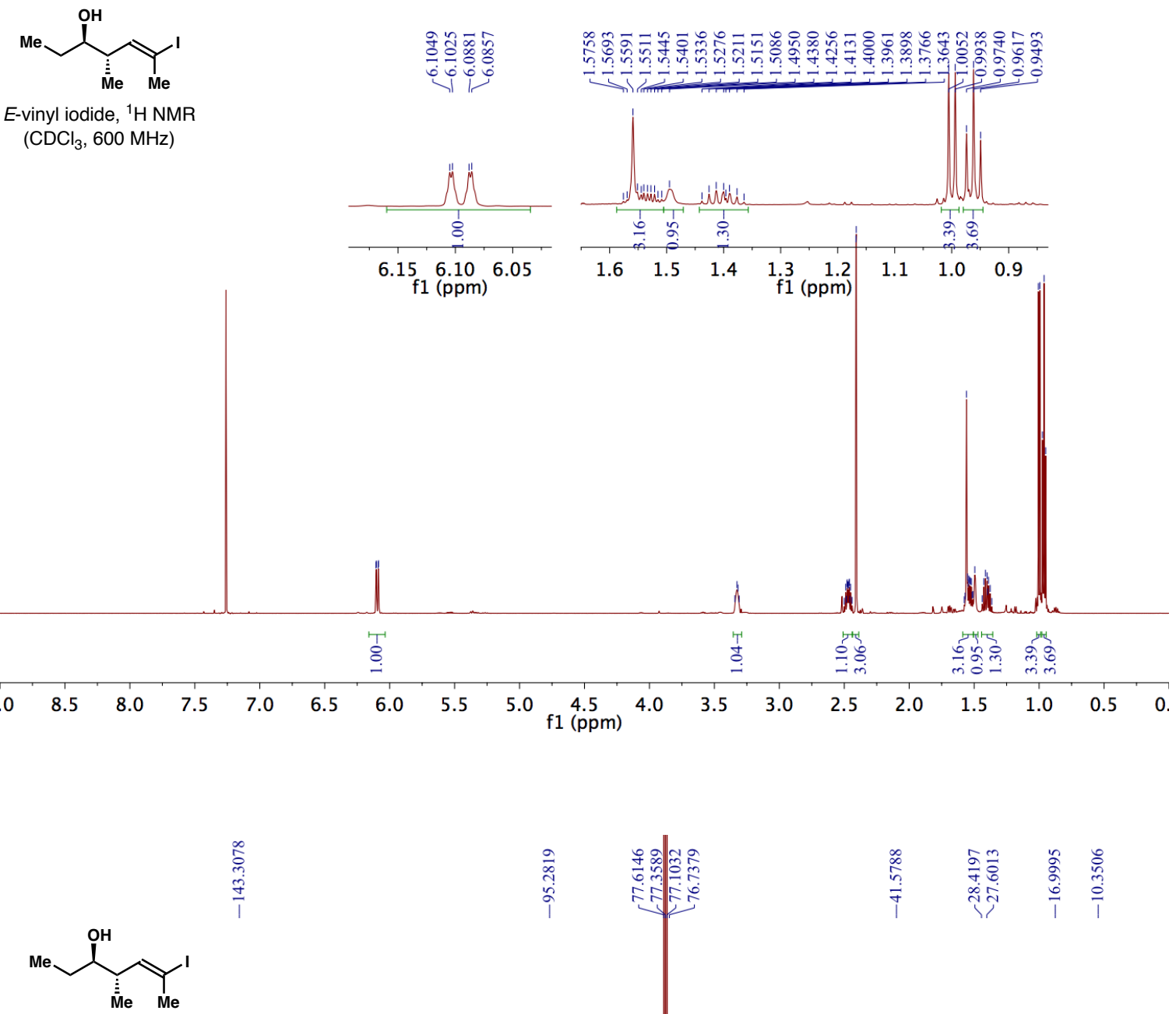

E-vinyl iodide, ${ }^{13} \mathrm{C}$ NMR $\left(\mathrm{CDCl}_{3}, 126 \mathrm{MHz}\right)$

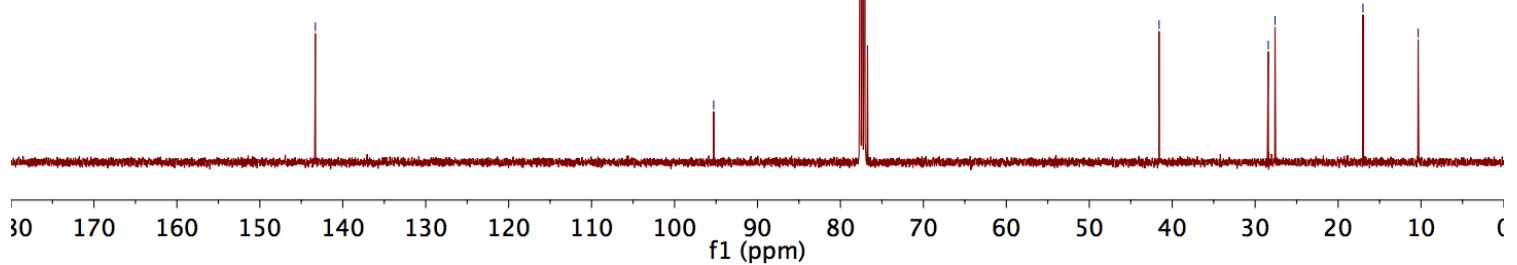



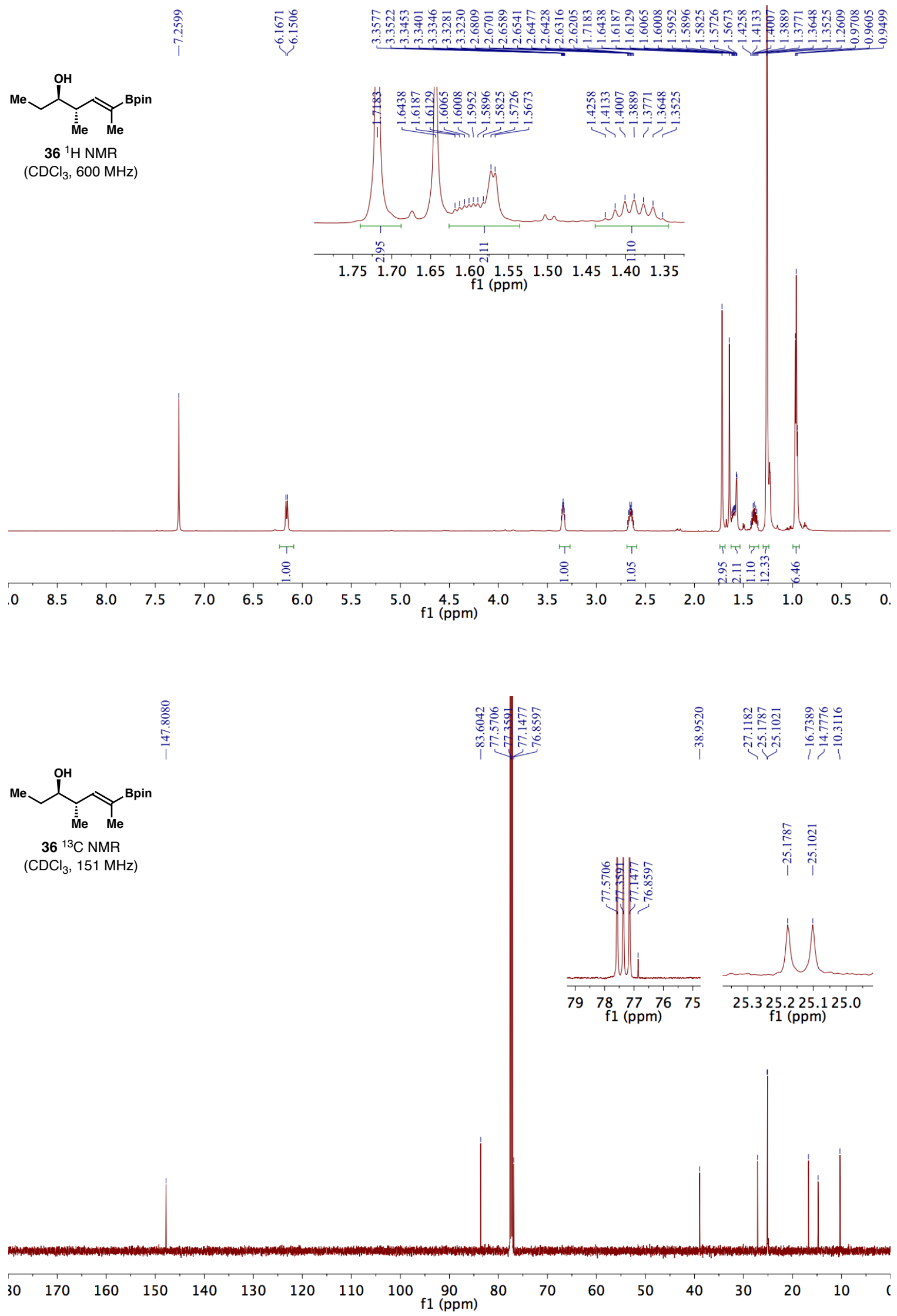

SI-116 

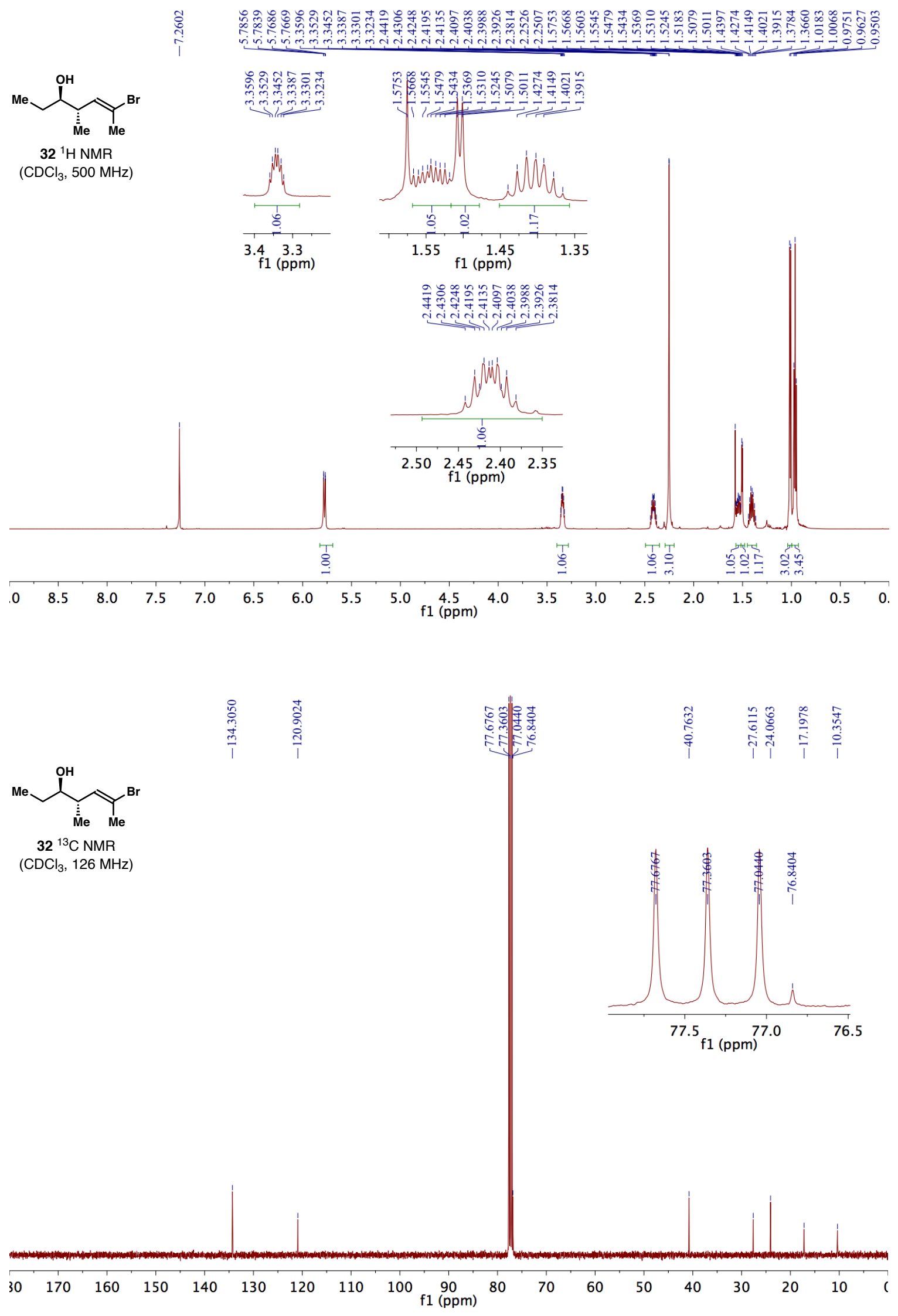


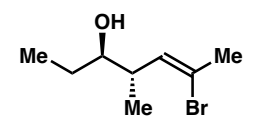

Z-vinyl bromide, ${ }^{1} \mathrm{H}$ NMR

$\left(\mathrm{CDCl}_{3}, 600 \mathrm{MHz}\right)$
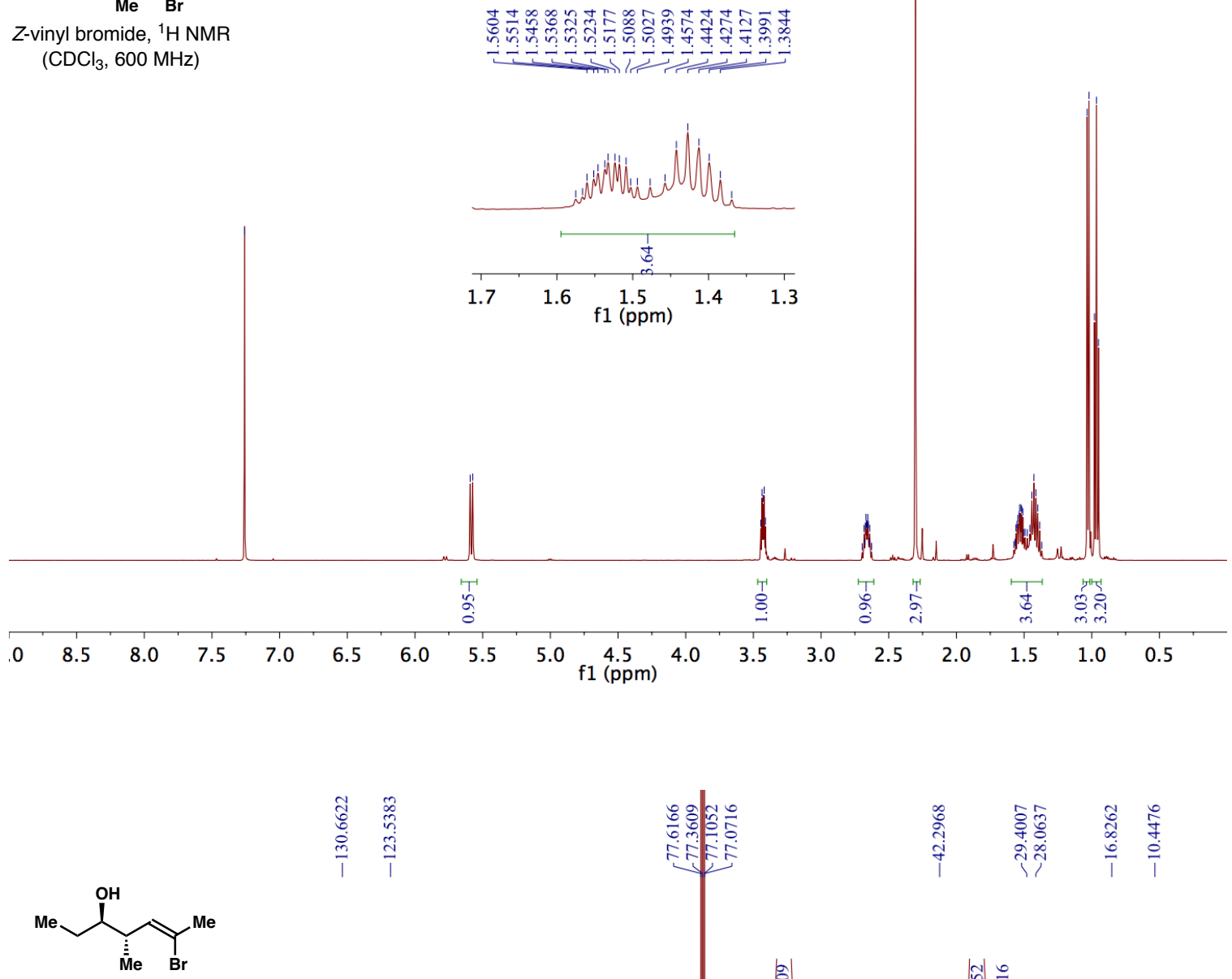

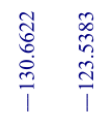

Z-vinyl bromide, ${ }^{13} \mathrm{C}$ NMR $\left(\mathrm{CDCl}_{3}, 101 \mathrm{MHz}\right)$

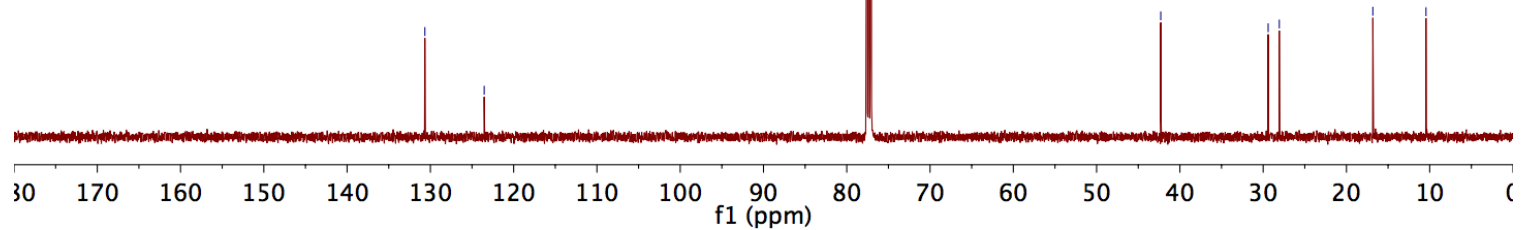



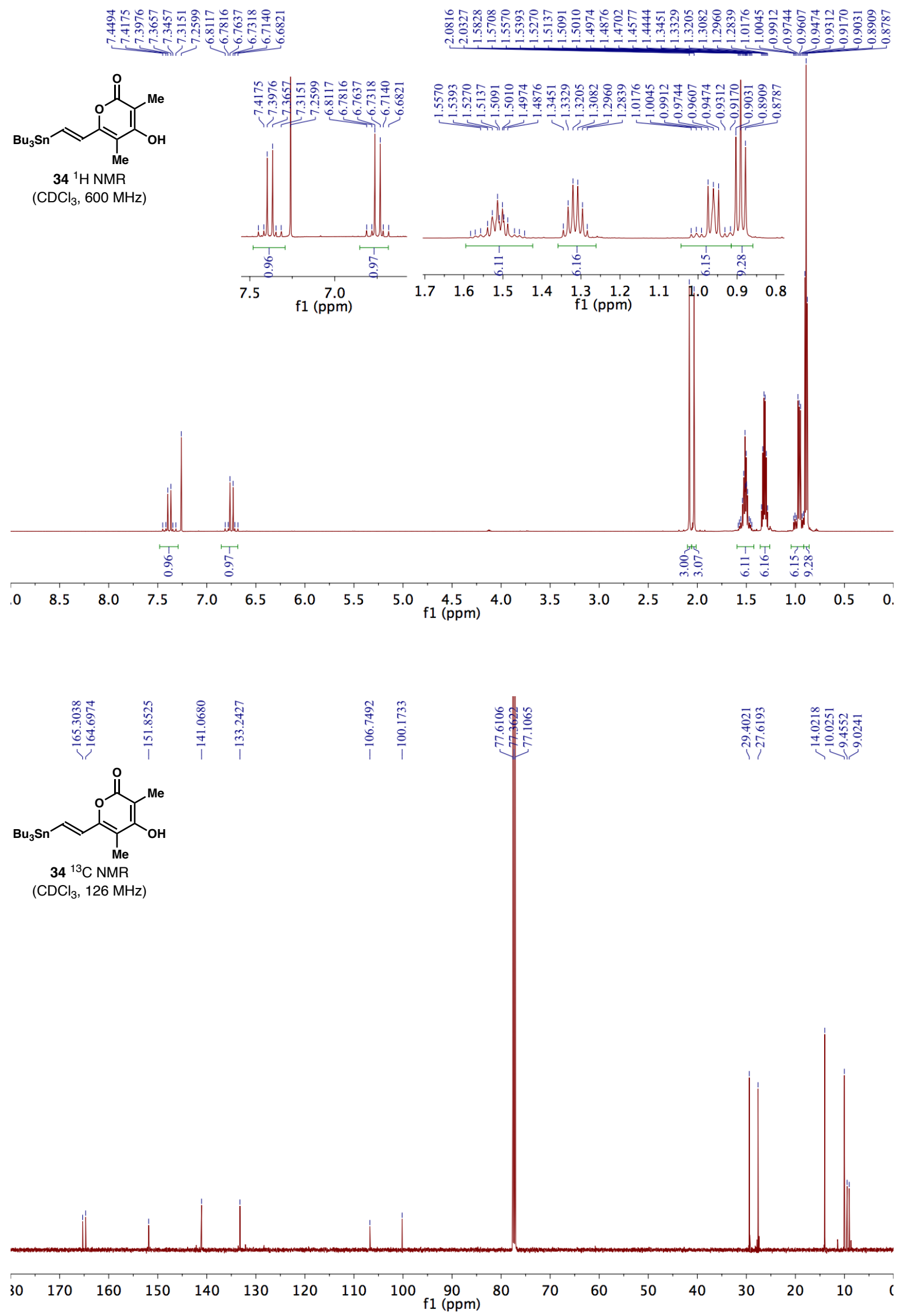

SI-119 

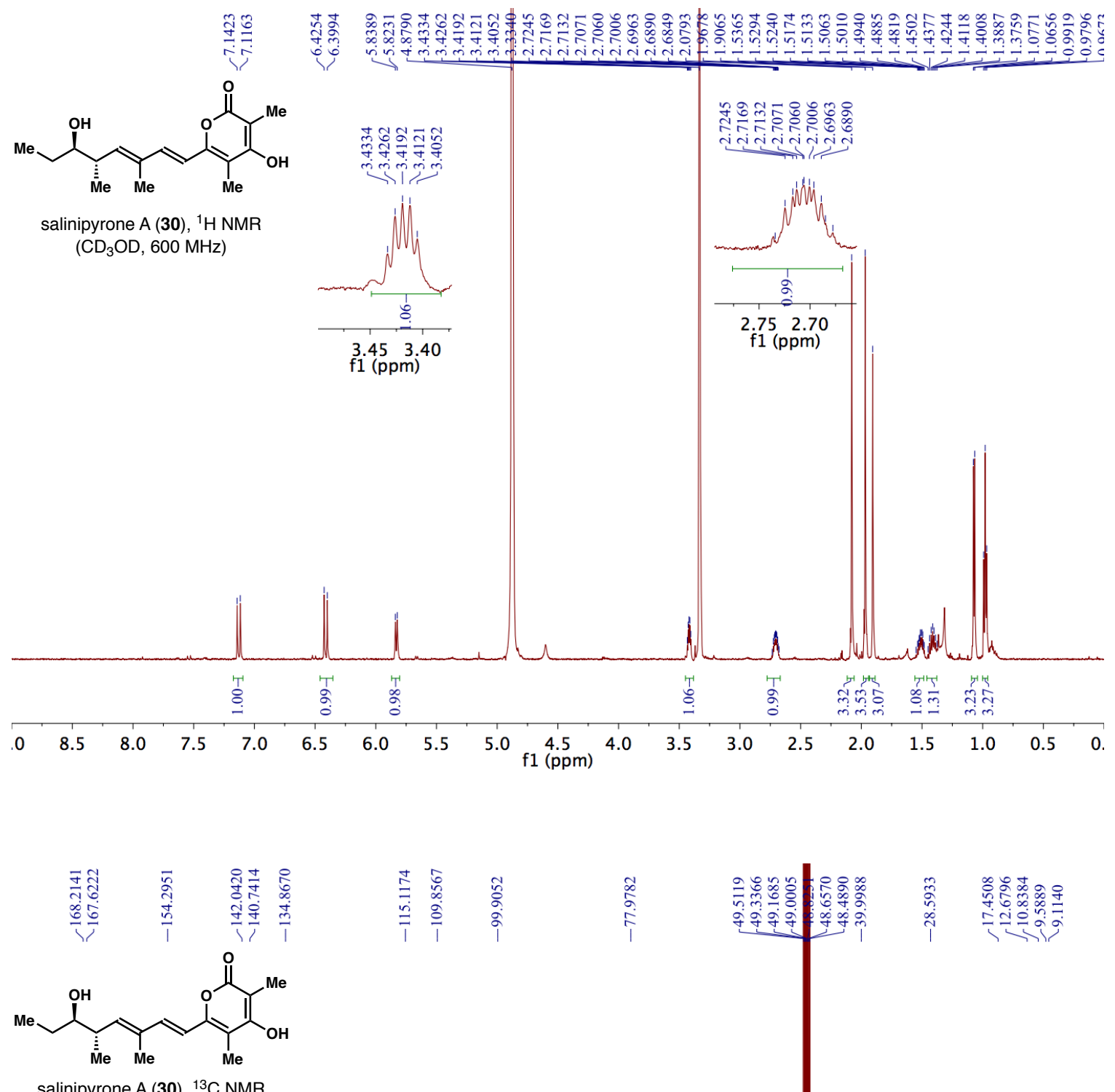

salinipyrone $A(30),{ }^{13} \mathrm{C}$ NMR

$\left(\mathrm{CD}_{3} \mathrm{OD}, 126 \mathrm{MHz}\right)$

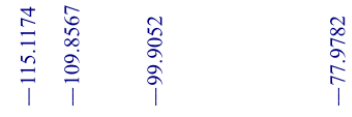

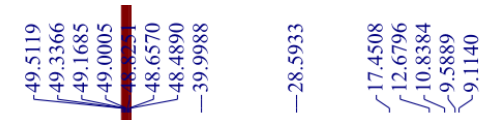



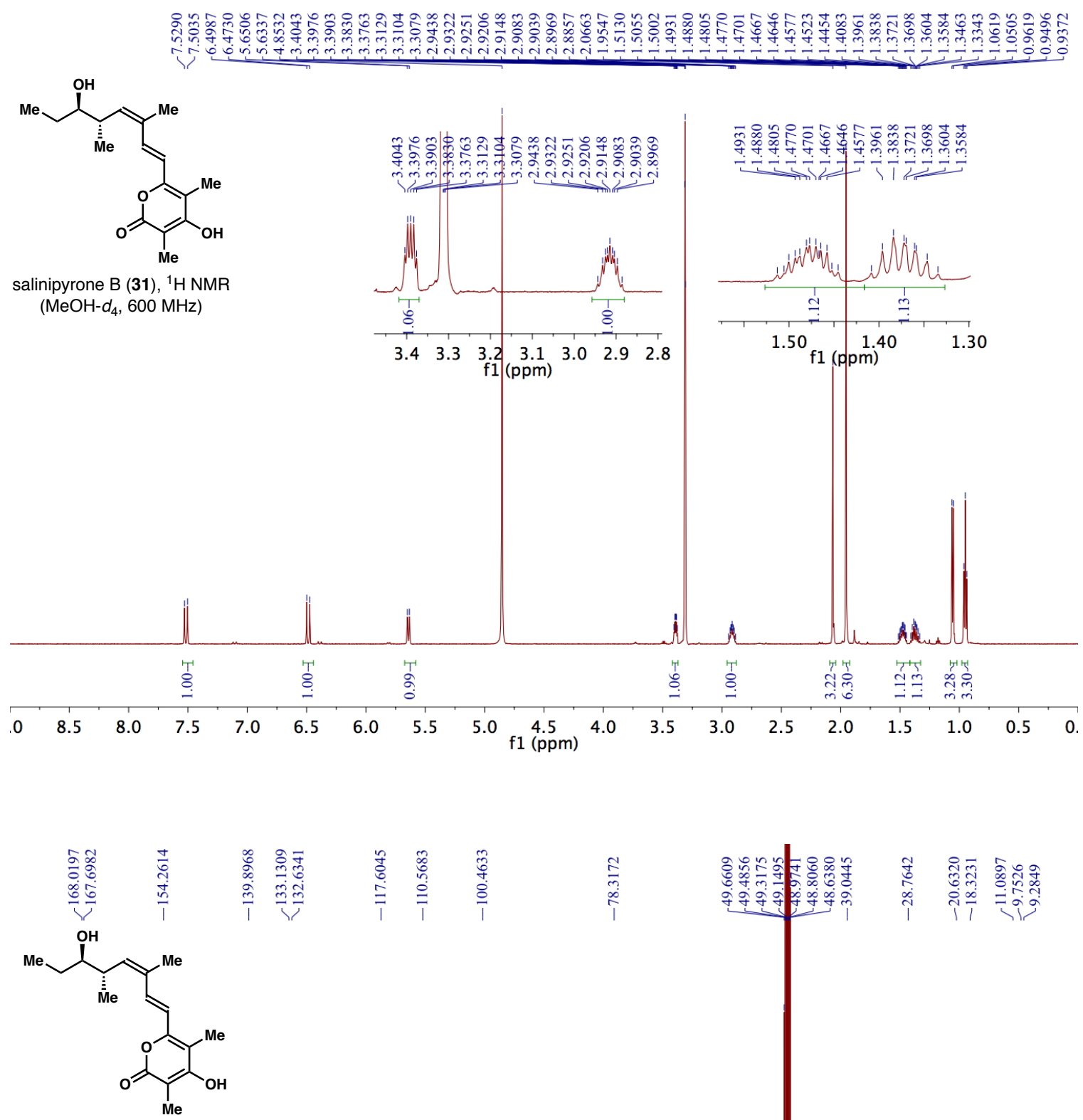

salinipyrone $B(31),{ }^{13} \mathrm{C}$ NMR $\left(\mathrm{MeOH}-d_{4}, 126 \mathrm{MHz}\right)$

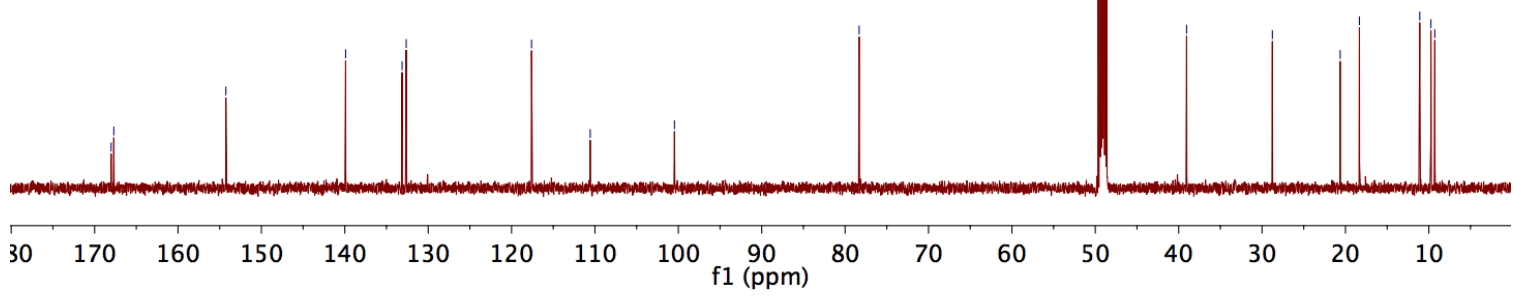


Comparison of ${ }^{1} \mathrm{H}$ and ${ }^{13} \mathrm{C}$ NMR Spectroscopic Data of Synthetic and Natural Salinipyrone A (Solvent: $\left.\mathrm{CD}_{3} \mathrm{OD}\right)$
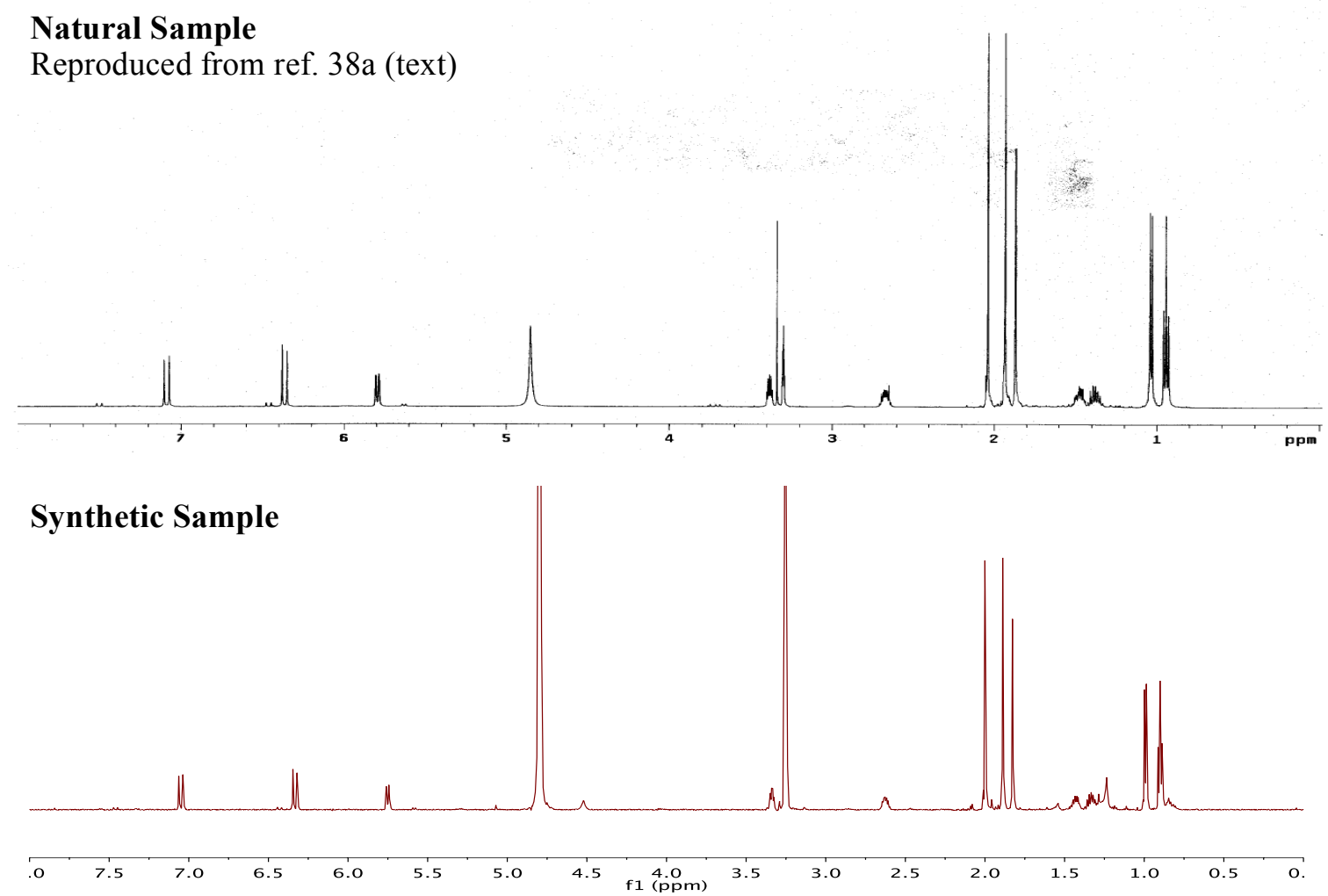

Natural Sample

Reproduced from ref. 38a (text)

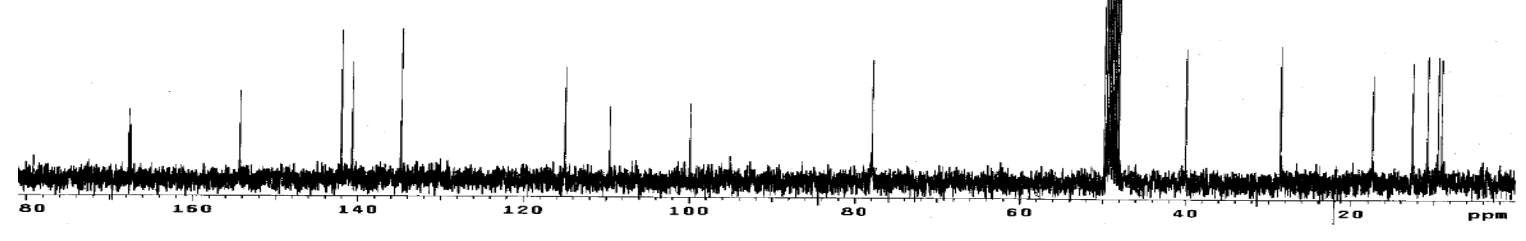

Synthetic Sample

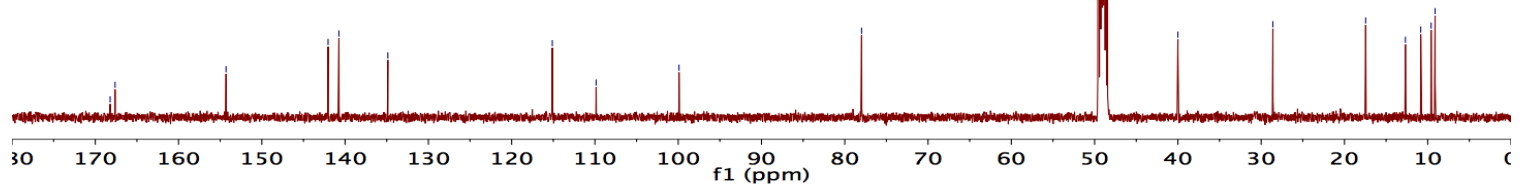


Comparison of ${ }^{1} \mathrm{H}$ and ${ }^{13} \mathrm{C}$ NMR Spectroscopic Data of Synthetic and Natural Salinipyrone B (Solvent: $\mathrm{CD}_{\mathbf{3}} \mathrm{OD}$ )

Natural Sample

Reproduced from ref. 38a (text)

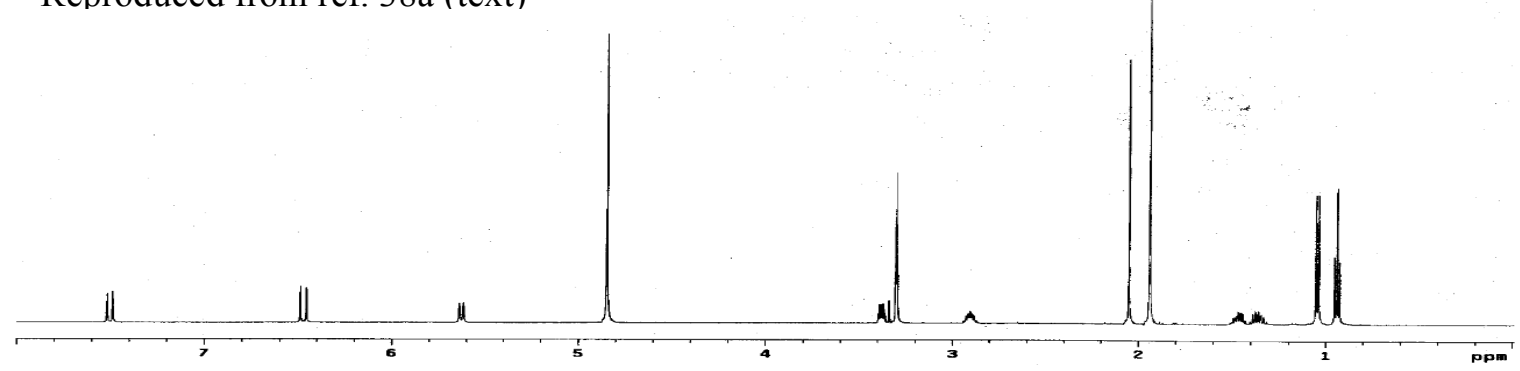

Synthetic Sample

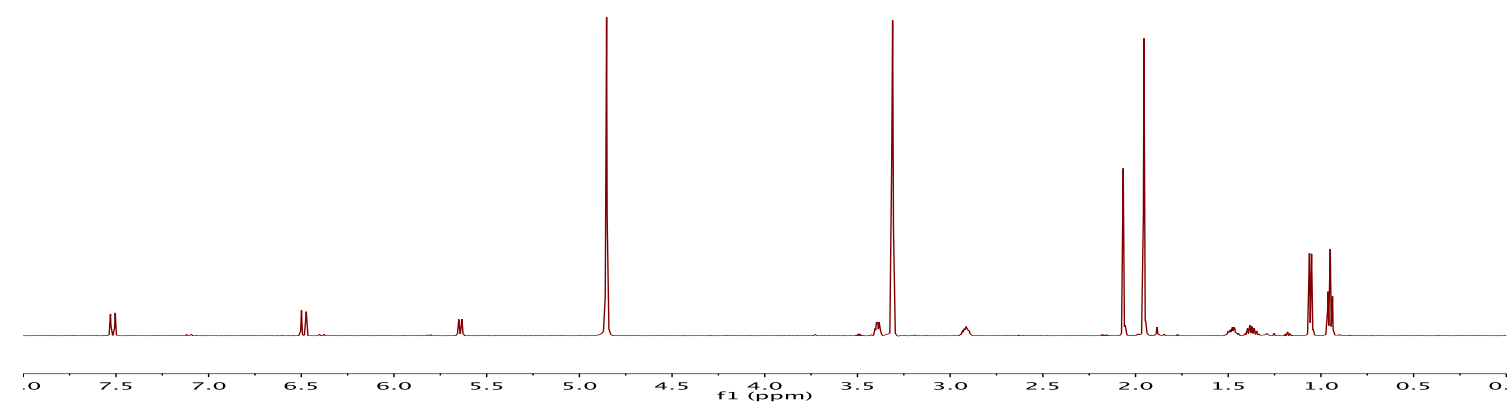

Natural Sample

Reproduced from ref. 38a (text)

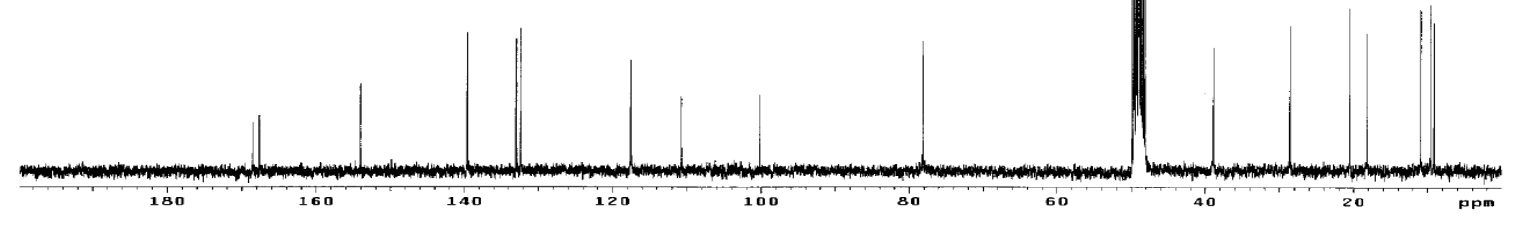

Synthetic Sample

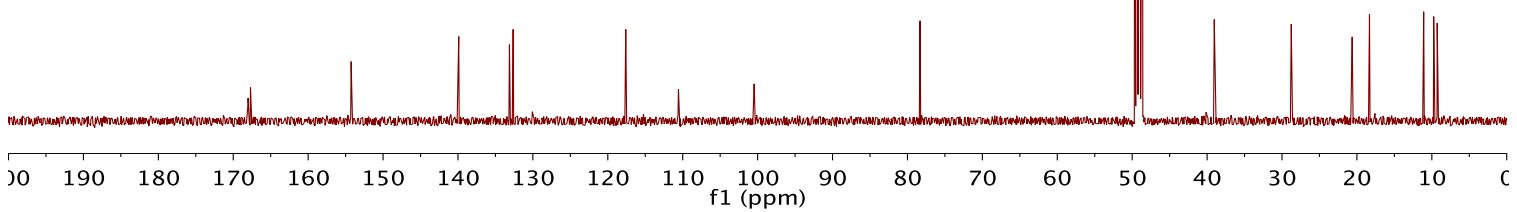

